\title{
Die Entwicklung der morphologischen Methode im Lappischen
}

\section{EINLEITUNG}

Die Forschung der letzten Jahrzehnte hat erwiesen, dass das Lappische in der uralischen Sprachfamilie den ostseefinnischen Sprachen am nächsten steht. Vor allem lautgeschichtlich, aber auch morphologisch, lassen sich das Ostseefinnische und das Lappische fast vollständig auf eine gemeinsame ursprachliche Form zurückführen, auf das Frühurfinnische, das sich in einiger Hinsicht unterscheidet von den ihm vorangegangenen Ursprachen (Ravila FUF 23 S. 20-65; Erkki Itkonen, Suomalaisugrilaisen kielen- ja historiantutkimuksen alalta [Zur finnischugrischen Sprach- und Geschichtsforschung] S. 97-99). Umso erstaunlicher ist, dass man bereits nach oberflächlicher Überprüfung feststellen kann, dass das Lappische und besonders dessen östliche Dialekte hinsichtlich der morphologischen Typologie stark abweichen vom Finnischen und von den übrigen finnisch-ugrischen Sprachen. Charakteristisch für die übrigen finnisch-ugrischen Sprachen ist, dass die Wörter allgemein leicht in Morpheme segmentiert werden können, die oft ganz mechanisch miteinander verbunden werden, dass der morphophonemische Wechsel gering ist und die Morphemgrenze nur selten verdeckt. Im Lappischen begegnet dagegen reichlich ein nichtautomatischer morphophonemischer Wechsel, und die meisten Dialekte besitzen eine typische innere Flexion, die grammatischen Verhältnisse werden ohne Suffixe ausgedrückt, durch eine Veränderung des Stammes lediglich. Vergleichen wir z.B. die folgenden skoltlappischen Formpaare mit ihren finnischen Entsprechungen: 
LpSk. (Snk.)

лop̄p ${ }^{E}$ 'Vogel' - Dem. Gen. Sg. ^กั๊̆Da

2. Sg. Imp. jē $\eta^{E}-3$. Pl. Prät. jellle 'leben'

3. Pl. Prs. mạnṇ̃e - 3. Pl. Prät. meññe 'gehen'
Fi.

lintu - lintu-se-n id.

$e l \ddot{a}-e l-i-v \ddot{a}-t \mathrm{id}$.

Und doch hat das Frühurfinnische als eine ziemlich rein agglutinierende Sprachform zu gelten. Die Forscher dürften heute darin übereinstimmen, dass z.B. die oben genannten skoltlappischen Formen auf Morphemreihen zurückgehen, die mehr an finnische als an skoltlappische Formen erinnern (frühurlp.

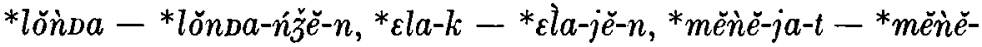
$j e-n)$. Wie ich bereits früher festgestellt habe (MSFOu 143 S. 16-22, Vir. 1967 S. 353-358), ist der Wandel in der lappischen Morphologie nach der frühurfinnischen Zeit eingetreten und er beruht darauf, dass ursprünglich völlig mechanische Lautwechsel im Innern des Stammes distinktiv wurden und morphologische Funktionen erhielten. Die vorliegende Untersuchung soll klären, in welchem Umfang die paradigmatischen Wechsel im Stamminnern sowie an der Grenze von Stamm und Suffix in den lappischen Dialekten in morphologischer Funktion begegnen und wie der Wandel der morphologischen Methode diachronisch zu verstehen ist. Eingangs wird die Typologie der lappischen Dialekte verglichen mit den übrigen finnisch-ugrischen Sprachen untersucht. Es folgt der Nachweis, welche Lautwechsel morphologisch relevant wurden, und welche morphologischen Verhältnisse durch die einzelnen Lautwechsel und ihre Kombinationen in den verschiedenen Mundarten ausgedrückt werden. Nach dem Abschnitt über die Beziehung zwischen kombinatorischem Lautwandel und veränderter morphologischer Methode wird abschliessend erörtert, ob sich die erzielten Ergebnisse auf andere Sprachen anwenden lassen.

Bei der typologischen Klassifizierung der Sprachen nahmen morphologische Kriterien stets einen wichtigen Platz ein. Das gilt schon für die im 19.Jh. von August Wilhelm und Friedrich 
von Schlegel, Wilhelm von Humboldt und anderen entwickelte Klassifikation, wo die Sprachen nach morphologischen Prinzipien eingeteilt wurden in isolierende, agglutinierende, flektierende und polysynthetische (s. Otto Jespersen, Language, London 1922, S. 76-80; Erkki Itkonen, Kieli ja sen tutkimus [Sprache und Sprachforschung], Helsinki 1966, S. 332-335; Aulis J. Joki, Maailman kielet [Die Sprachen der Welt], Helsinki 1966, S. 15-18). Wesentlich auf der Morphologie basieren auch die bedeutendsten Typologien aus diesem Jahrhundert, obwohl man bestrebt war, auch syntaktische und phonologische Faktoren zu berücksichtigen. Edward Sapir teilt die Sprachen ein nach der Beschaffenheit der grammatischen Begriffe, der morphologischen Methode und dem Grad der Synthese (Language 1. Aufl. 1921, hier wird die Aufl. von 1966 benutzt, S. 120-146). Da sich der im Lappischen eingetretene typologische Wandel gerade auf die morphologische Methode bezieht, beschäftigen wir uns hier nur mit dieser Klassifizierung näher.

Unter Methode versteht Sapir das Verfahren, wonach die Wurzelelemente und die grammatischen Elemente eine Verbindung eingehen. Er unterscheidet vier Methoden: Isolation, Agglutination, Fusion und Symbolismus. Die Klassen zeigen eigentlich, wie eng die Elemente jeweils miteinander verbunden sind. Isolierende Sprachen haben keine Flexion, und das Wort ist immer identisch mit dem Wurzelelement. In agglutinierenden Sprachen verbinden sich die grammatischen Elemente relativ locker und ohne lautliche Veränderungen mit den Wurzelelementen, bei der Fusion entsteht eine festere Kupplung zwischen Wurzel und Affix, das symbolische Verfahren drückt die grammatischen Verhältnisse durch innere Lautwechsel des Wurzelelementes aus, welche die Qualität, die Quantität, den Akzent und die Betonung von Lauten beeinflussen können. Nach Sapir gehört auch die Reduplikation zum Symbolismus. Psychologische Faktoren sind Sapir offenbar sehr wichtig als Kriterien für den Grad der Festigkeit von Verbindungen. Er meint z.B., das Vorhandensein von Numerusverhältnissen im Englischen wie deer:deer, ox:oxen, goose: geese neben der regelmässigen Pluralbildung (book $:$ books) lasse die Verbindung des Stammes book und des Suffixes $s$ fester erscheinen als es 
beim Fehlen von unregelmässigen Pluralformen der Fall wäre. "If the form pattern represented by the word books is identical, as far as use is conserned, with that of the word oxen, the pluralizing elements $-s$ and -en cannot have quite so definite, quite so autonomous, a value as we might at first be inclined to suppose. They are plural elements only in so far as plurality is predicated of certain selected concepts. The words books and oxen are therefore a little other than mechanical combinations of the symbol of a thing (book, $o x$ ) and a clear symbol of plurality." (a.a.O. 131-132). Der Mangel von genauen formalen Kriterien bewirkt, dass die Grenzen zwischen Agglutination, Fusion und Symbolismus nicht immer ganz klar sind. Die Festigkeit der Verbindung weist gewisse Zwischenstufen auf. Im Wort good-ness zeigt sich eine klare Agglutination, bei book-s haben wir es mit einer regelmässigen Fusion zu tun, bei depth (Wurzel deep) mit einer unregelmässigen Fusion und bei geese (Wurzel goose) liegt Symbolismus vor.

Sapir betont eigens, dass die Sprachen normalerweise eine bestimmte Methode, ein Verfahren, nicht in Reinkultur vertreten, sondern in ein und derselben Sprachen begegnen die einzelnen Methoden mehr oder weniger nebeneinander. Schwierig wird nun in der Praxis die Unterscheidung: wie ist der Anteil der einzelnen Methoden abzuwägen, wonach ist zu klassifizieren? Wieviel Fusion und Symbolismus ist zulässig für eine agglutinierende Sprache? Wieviel Symbolismus muss eine Sprache aufweisen, damit sie als symbolisch bezeichnet werden kann? Definitionen besagten Verfahrens bei Sapir wie agglutinierendfusionierend, agglutinierend-isolierend, symbolisch-fusionierend, isolierend (leicht agglutinierend) usw. vermögen nur ein sehr oberflächliches Bild zu geben vom Anteil der einzelnen Methoden.

Die in Sapirs Typologie auftretenden Probleme werden in der von Joseph H. Greenberg ( $I J A L 26$ 1960) teilweise rermieden. Die charakteristischen Eigenschaften der Sprache werden darin ausgedrückt durch numerische Verhältniszahlen, durch Indexe. Die Formmethode wird in erster Linie bestimmt durch den Agglutinationsindex $\mathrm{A} / \mathrm{J}$, wo $\mathrm{A}=$ die Anzahl der agglutinierenden Konstruktionen und $\mathbf{J}=$ die Anzahl der Morphemgren- 
zen besagt. Die sonstigen Indexe sind: der Index der Synthese $\mathrm{M} / \mathrm{W}$ ( $\mathrm{M}=$ Anzahl der Morpheme, $\mathrm{W}=$ Anzahl der Wörter), der Index der Komposita $\mathrm{R} / \mathrm{W}$ ( $\mathrm{R}=$ Anzahl der Wurzelmorpheme), der Index der Derivation $\mathrm{D} / \mathrm{W}(\mathrm{D}=$ Anzahl der Ableitungsmorpheme), der Index der Inflexion I/W ( $=$ Anzahl der Beugungs- bzw. Inflexionsmorpheme), der Index der Präfixe $\mathrm{P} / \mathrm{W}(\mathrm{P}=$ Anzahl der Präfixe), der Index der Suffixe (S/W $(\mathrm{S}=$ Anzahl der Suffixe), der Index der Isolierung $\mathrm{O} / \mathrm{N}(\mathrm{O}=$ Anzahl der Fälle mit distinktiver Wortfolge, $\mathrm{N}=$ Anzahl der Nexus), der Index der blossen Inflexion $\mathrm{Pi} / \mathrm{N}(\mathrm{Pi}=$ Anzahl der nichtkongruenten Inflexionsmorpheme) und der Index der Kongruenz $\mathrm{Co} / \mathrm{N}(\mathrm{Co}=$ Anzahl der Kongruenzfälle). Greenberg bringt in seinem Artikel die von ihm berechneten Indexe für das Sanskrit, das Angelsächsische, das Persische, das Englische, das Jakutische, das Suaheli, das Anamitische und das Eskimoische. Die Berechnungen wurden an Textproben in einer Länge von nur hundert Wörtern vorgenommen (a.a.o. 193).

In Greenbergs Typologie werden die Sprachen ihrer Formmethode nach auf einer eindimensionalen Skala untergebracht, wo der Agglutinationsindex den Platz einer jeden Sprache bestimmt. Theoretische Grenzwerte bilden 1 - eine rein agglutinierende Sprache - und 0 - eine Sprache ohne jegliche Agglutination. Fusion und Symbolismus bedeuten lediglich fehlende Agglutination, so dass die Bestimmung dieser Eigenschaften in Greenbergs System entfällt. In Fällen mit niedrigem Agglutinationsindex bleibt unklar, um was für eine morphologische Methode es sich handelt, wie tiefgehende Veränderungen die $\mathrm{Fu}$ sion in der Phonemgestalt der Morpheme verursacht, ob sich Affixe unterscheiden lassen oder ob sich Flexion und Ableitung mit Hilfe von Wechseln in Innern des Stammes vollziehen. Zwar meint Greenberg, der Symbolismus sei eigentlich nichts anderes als die Verwendung von Infixen. Im engl. Paradigma sing : sang: sung 'singen' z.B. begegnen die Infixe $i, a[e]$ und $u[A]$. Diese Auslegung ist vielleicht zu verteidigen zumindest in den Fällen, wo der distinktive Teil deutlich segmentierbar ist, vgl. mit dem engl. Beispiel das Formpaar lpKo (Snk.) 3. Pl. Prs. màñne : 3. Pl. Prät. meñne 'gehen', wo die erstere Form 
nach Greenbergs Analyse das Infix $\dot{a}$ als Charakter haben könnte und die letztere das Infix e. Komplizierter wird die Sache aber, wenn man die genannten skoltlappischen Formen als Teile des gesamten Paradigmas sieht. Die Form der 3. Sg. Prs. $m a ̄ n n n s$ unterscheidet sich von den obigen ausser durch das "Infix" $\bar{a}$ auch durch das "Infix" $\check{n} n$ und das Suffix $\stackrel{A}{>}$. Die Form der 2. Sg. Imp. $m \bar{\theta} n n_{<}^{A}$ unterscheidet sich hiervon wiederum durch die "Infixe» $\bar{\theta}$ und $n$ und den erhaltenen Auslautvokal des Stammes. In der Formreihe 1. Sg. Prs. mōnam : 1. Sg. Prät.

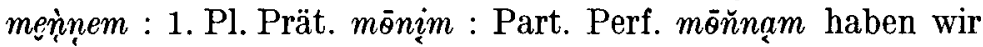
ausser den anstelle des Auslautvokals des Stammes stehenden Suffixen $e: \underset{i}{i}: a$ als distinktive Faktoren auch die "Infixe» $\vec{\theta}: e$, $n: \grave{n} n: \breve{n} n$. In solchen Fällen müsste man als Charakteristik einer jeden Form eine ganze Reihe von Infixen und Suffixen anführen. Oft wäre es vielleicht am vorteilhaftesten, die einem Wechsel unterworfenen Stellen als suprasegmentale Morphe zu behandeln, vom Vokal der ersten bis zum Vokal der zweiten Silbe gehend. Das gilt z.B. für solche Reihen von $1 p N$ wie 2. Sg. Imp. gò-arù : 3. Sg.Prs. goarru : Part.Prs. gŏă·rrru 'nähen', wo die die ganze Wortgestalt betreffende Quantität distinktiv ist (Ravila Vir. 1961 S. 347). Es fragt sich, ob derartige Morphe noch Infixe genannt werden können, auch wenn man zugeben würde, dass an einen Stamm gelangende Infixe ebenfalls verschiedene morphophonemische Veränderungen in beiden Morphemen hervorrufen können. Meines Erachtens kann man mit Sapir weiterhin dann von Symbolismus sprechen, wenn die Beugung (Inflexion) der Wörter oder die Ableitung so geschieht, dass sich ein wesentlich zum Stamm gehörendes Element qualitativ oder quantitativ verändert oder dass die Akzent- oder Betonungsverhältnisse des Stammes oder die Reduplikation eine morphologische Funktion haben.

In der für die Errechnung der Indexe nötigen Morphemanalyse folgt Greenberg hauptsächlich der auf der Distribution beruhenden strukturalistischen Methode, wie sie bis zu Beginn der sechziger Jahre in Amerika vorherrschend war. Die Schwächen von Greenbergs Typologie decken sich denn auch im grossen ganzen mit denen der Distributionsanalyse. Manche Begriffe sind zu vieldeutig. Von den Kritikern ist denn auch darauf 
hingewiesen worden, dass die Zuverlässigkeit der Resultate bei einer Anwendung der Greenbergschen Methode vor allem davon abhängt, wie eindeutig die Begriffe definiert werden können (vgl. I. I. Revzin, Models of Language, London 1966, S. 54-56; russ. Original Модели языка, 1962). Problematisch ist z.B. die Stellung der Null, die in der Morphologie etwas unbestimmt geblieben ist. Für das Resultat ist es jedoch nicht gleichgültig, ob z.B. die 3. Sg.Prät. $\operatorname{lpN}$ gulâi 'hören' so analysiert wird, dass ausser dem Stamm gulâ und dem Präteritumcharakter $i$ auch die Morpheme des Sg. und der 3. P. enthalten sind, in Form einer Leerstelle, Nullstufe $(g u l \hat{a}+i+O+O)$ oder eins von beiden $(g u l \hat{a}+i+O)$, oder aber so, dass nur zwei Morpheme darin vorhanden sind $(g u l \hat{l}+i)$. Je nach verwendeter Analyse schwankt die Anzahl der Morpheme von zwei bis vier und die der Morphemgrenzen von eins bis drei. Da Wörter dieser Art in lappischen Texten häufig sind, wird der Wert der Indexe durch die Wahl entscheidend beeinflusst.

Als annähernde Bedingungen für die Verwendung von Null dürften die folgenden von W. Haas angeführten gelten können (in dem Artikel Zero in Linguistic Description, Studies in Linguistic Analysis, Oxford 1958, S. 33-53):

1. Null muss mit einer offenkundigen Form abwechseln.

2. Null muss mit einer offenkundigen Form kontrastieren.

In unserem Beispiel (lpN gulâi) scheint das Morph der 3. P. die genannten Bedingungen zu erfüllen: Null steht im Wechsel mit einem offenkundigen Allomorph $s$, das im Imperativ begegnet (3. Sg.Imp. gullu-s) und ist in Opposition mit den offenkundigen Morphen der 1. und 2. P. (1. Sg.Prät. gulli-m, 2. gul$l i-k)$. Fraglich bleibt aber, ob die 3. P.Imp. ohne weiteres mit der 3. P. der anderen Modi vergleichbar ist. Im Indikativ, im Potentialis und im Konditionalis kann die 3. P. als kennzeichenlos angesehen werden, während die 1. und 2. P. einen Charakter besitzen, was u.a. daraus hervorgeht, dass die 3. P. in diesen Modi nie ein offenkundiges Morph hat (nach der Analyse von Greenberg müsste z.B. die Endung -gâa, -gă der 3. Du.Prät. und Kond. als Morphem des Dual und nicht der 3. P. angesehen werden, denn deren Allomorph -ka begegnet in der 3. Du. Imp. nach dem Personalmorphem $s$ ausschliesslich als Dualcharak- 
ter; wir kämen also zu der Matrix gulâ-i: gulâ-i-gâ, gull-u-s : gull-u-s-kâ), die 1. und 2. P. aber werden durch Suffixe charakterisiert. Im Imperativ würde die Lage wesentlich anders aussehen als in den sonstigen Modi. Ohne Gewaltanwendung wird man wohl zu keinem anderen Ergebnis kommen, dass im Imperativ die 2. P. und speziell die 2. P.Sg. keinen Charakter hat, während die übrigen einen besitzen. Dies geht eindeutig schon aus der Struktur wenigstens der finnisch-ugrischen und indoeuropäischen Sprachen hervor. Verlangt es einen nach anderen Kriterien, kann man sich darauf beziehen, was Joseph H. Greenberg in seiner interessanten Arbeit Language Universals (The Hague 1966) über die Frequenzen der Kategorien mit und ohne Kennzeichen festgestellt hat. Anhand zahlreicher Beispiele weist er nach, dass die Belegdichte einer Kategorie ohne Kennzeichen generell grösser ist als die einer mit Kennzeichen (a.a.O. 31-55). Auch aufgrund dieses Kriteriums ist zumindest im Lappischen die 2. P. im Imperativ und die 3. P. in den übrigen Modi kennzeichenlos. Um mir ein Bild machen zu können über die Frequenzverhältnisse habe ich sämtliche Personalformen des Ind., Pot. und Kond. auf den Seiten $9-29$ von Teil II (Texte) des Lehrbuchs Lærebok i lappisk von Konrad Nielsen exzerpiert. Ich habe diesen Abschnitt deshalb gewählt, weil er lediglich aus Dialogen besteht, die Situationen des alltäglichen Gesprächs gut wiedergeben und worin die gegenseitigen Frequenzverhältnisse der Personalformen offenbar der Wirklichkeit entsprechen. Es handelt sich bei dem Material um insges. 2151 Personalformen des Ind., Pot. und Kond. $23 \%$ davon entfallen auf Formen der 1. P., $10 \%$ auf Formen der 2. P. und $67 \%$ auf Formen der 3. P. Die Gesamtfrequenz der Imperativformen ist so gering, dass ich, um eine genügende Erhebungsauswahl zu erreichen, sämtliche Gespräche durchgehen musste, die auf den Seiten 9-109 des genannten Buches stehen. Von den dadurch erhaltenen 247 Imperativformen sind $89 \%$ Formen der 2. P. Die 2. P. überwiegt derart, dass auch eine grössere Erhebungsauswahl wahrscheinlich keine wesentliche Änderung für die Frequenz brächte.

Aufgrund des oben Gesagten wäre es willkürlich, für $\operatorname{lpN}$ ein Morphem der 2. P. anzunehmen, das sich im Sg. des Ind., Pot. 
und Kond. als $k$ realisieren würde, im Imp.Sg. aber als Null, und ein Morphem der 3. P., das sich im Sg. des Ind., Pot. und Kond. als Null realisiert, im Imp.Sg. aber als $s$. Man hat im Hinblick auf die Tatsachen zwangsweise davon auszugehen, dass im Ind., Pot. und Kond. die 3. P., im Imp. aber die 2. P. kennzeichenlos sind und kein grammatisches Yorphem besitzen, während die 1. und 2. Ind., Pot. und Kond. sowie die 1. und 3. Imp. ein Kennzeichen haben. ${ }^{1}$ Somit kommen wir zu dem Schluss, dass die Form lpN gulâi kein Personalmorphem hat, das als Nullstufe vertreten wäre.

Auch die Frage von der Existenz eines Singular-JIorphems und dessen Null-Allomorphs ist problematisch. Das Null-Allomorph erfüllt die eine der Bedingungen von Haas: es steht in Opposition mit den offenkundigen Charakteren des Dual und des Plural (3. Du. Prät. gulâ-i-gâ, 1. Pl. gulâ-i-me-k). Dagegen scheint es zumindest auf den ersten Blick die erste Bedingung nicht zu erfüllen. Man kann aber trotzdem auch den Standpunkt vertreten, dass das Null-Allomorph des Sg. mit einem offenkundigen Allomorph wechselt. Man kann behaupten, bei der Flexion des Verneinungsverbs (lpN 1. Sg. im, 2. $i k, 3 . i$,

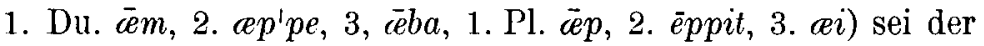
Singularcharakter das $i$, das anstelle des Stammvokals $a \sim e$ auftritt. Begründen lässt sich diese Behauptung u.a. damit, dass die Opposition zwischen den Numeri in den Formen 1. Sg. im und 1. Du. $\bar{e} m$ lediglich hervorgeht aus dem Vokalwechsel $i \sim c$. Das Singularmorphem auf ein in der Flexion eines einzigen Wortes begegnendes offenkundiges Allomorph zu bauen, ist jedoch gekünstelt, da für alle anderen Zusammenhänge ein Null-Allomorph angenommen werden müsste. Sol Saporta hat die von Haas angeführten Bedingungen kritisiert und die erste Bedingung durch einen Zusatz ergänzt, dass nämlich die mit Null wechselnde offenkundige Form produktiv sein müsse oder

${ }^{1}$ Wenigstens Warren Cowgill hat die Ansicht vertreten, in der 2. P.Sg. Imp.Akt. sei in den ieur. Sprachen die Null als Personal-Modus-Endung enthalten, auch wenn es eine offenkundige Endung der 2. Sg.Imp. nicht gäbe (in dem Artikel Universals in Indo-European Morphology, Universals of Languages, hrsg. Joseph H. Greenberg, Cambridge, Mass. 1963, S. 97). M.E. ist eine solche Auslegung nicht realistisch. 
zumindestens weniger marginal als das angenommene SingularMorphem in der Form engl. man (vgl. Pl. men) (Proc. of the 9. International Congress of Linguists, The Hague 1964, S. 228230). Kann aber etwas so Relatives wie die Produktivität, ganz $\mathrm{zu}$ schweigen von einer geringen Marginalität, als Kriterium dienen? Ist z.B. das $t$-Element, das im Nom.Sg. der lappischen Demonstrativpronomina regelmässig auftritt (lpN dät 'dieser', dât 'das', duot 'jener' usw., vgl. Gen., Akk. Sg. dām, dâm, duom usw.), "produktiv" genug, dass ein Morphem des Nom.Sg. angenommen werden könnte, dessen Allomorph sonst überall in der Flexion der Nomina Null wäre? Meines Erachtens muss hier die Praxis entscheiden. Die Nullstufe ist ein Hilfsbegriff, sinnvoll nur dann, wenn die Schilderung dadurch wesentlich vereinfacht und erleichtert wird. Wenn wir annehmen, dass es in der lappischen Konjugation ein Singular-Morphem gibt, als dessen Allomorph in der Flexion des Verneinungsverbs anstelle des Stammvokals ein $i$ auftritt und anderwärts Null, oder in der Deklination ein Nominativ-Morphem, dessen Allomorph im Singular der Demonstrativpronomina $t$ lautet und sonst durch die Nullstufe vertreten ist, leuchtet ohne weiteres ein, dass die Schilderung durch die Verwendung der Null hier eher kompliziert als vereinfacht wird. Man verzichtet lieber auf die Morpheme von Singular und Nominativ und betrachtet $i$, $d \bar{a} t, d a \hat{t}, d u o t$ usw. als Allomorphe eines lexikalischen Morphems.

Aufgrund des Vorhergehenden nehmen wir an, dass wir es in Fällen wie lpN gulâi mit zwei Morphemen zu tun haben, dem Stamm und dem Charakter des Präteritums.

Vor allem ist es der Begriff der Agglutination, der Schwierigkeiten bereitet bei der Anwendung der Greenbergschen Typologie auf die Praxis. Der Definition zufolge ist eine Konstruktion agglutinierend, wenn sich die Morpheme automatisch miteinander verbinden. Die Morpheme müssen eine konstante Form haben, nur automatischer Wechsel darf in ihrer morphophonemischen Gestalt zum Ausdruck kommen. Das Prinzip scheint eindeutig, doch steht man in der praktischen Analyse immer wieder vor Fällen, wo sich nicht unbedingt entscheiden lässt, ob der Wechsel automatisch ist oder nicht. Für viele, vielleicht für die meisten Sprachen kann man alternative morphophone- 
mische Deutungen anführen und oft entscheidet dann die Wahl einer bestimmten Deutung, ob ein Wechsel nun als automatisch angesehen wird oder nicht. Zweitens steht man oft vor der Frage, wie bedingungslos die Forderung des Automatischen hier aufzufassen ist. Der strengsten Deutung nach wäre z.B. der Stufenwechsel in der finnischen Hochsprache nichtautomatisch, da es Ausnahmen gibt wie Formen mit den Possessivsuffixen der 3. P. und der 1. und 2. P.Pl., z.B. patansa, patamme, patanne (vom Wort pata 'Topf') pro *padansa, *padamme, *padanne und Formen des Pass.Prs. wie saadaan, viedään (von den Verben saada 'bekommen', viedä 'bringen') pro *saataan, *vietään (vgl. Pass.Prät. saatiin, vietiin). Wenn der Stufenwechsel dieser Ausnahmen wegen als nichtautomatisch angesehen wird, sind alle Konstruktionen, deren eine Komponente ein dem Stufenwechsel unterliegender Nominal- oder Verbalstamm ist, nichtagglutinierend.

Warren Cowgill hat Zweifel geäussert, ob ein Text von 100 Wörtern lang genug sei für ein zuverlässiges Resultat (a.a.0. 93, vgl. auch Greenberg $I J A L 26$ S. 194 und Erkki Itkonen, Kieli ja sen tutkimus S. 340). Die Glaubwürdigkeit der Indexe hätte durch Berechnung des mittleren Fehlers festgestellt werden können. Weder Greenberg noch Cowgill haben das jedoch getan. Alle Indexe sind an sich Mittelwerte, durch die ausgedrückt wird, wieviel Morpheme, Wurzeln, Ableitungsmorpheme usw. durchschnittlich in einem Wort enthalten sind, wieviel agglutinierende Konstruktionen im Durchschnitt auf eine Morphemgrenze kommen oder wieviel distinktive Wortfolgefälle, von der Kongruenz unabhängige Flexionsmorpheme oder Kongruenzfälle durchschnittlich auf einen Nexus entfallen. Der mittlere Fehler der Indexzahlen lässt sich somit nach dem Schema des mittleren Fehlers des Mittelwertes errechnen

$$
\varepsilon(\mathrm{K})=\frac{\sigma}{\sqrt{\mathrm{N}-1}},
$$

wo $\varepsilon$ der mittlere Fehler ist, $\mathrm{K}$ der Mittelwert, $\sigma$ der Wert der quadratischen Streuung und $\mathrm{N}$ die Gesamtfrequenz der Fälle (Wörter, Morphemgrenzen oder Nexus). Die quadratische Streuung wird errechnet nach dem Schema 


$$
\sigma=\sqrt{\frac{\Sigma f x^{2}}{N}}
$$

wo f die Klassenfrequenz bezeichnet und $\mathrm{x}$ die Abweichung vom Mittelwert (beim Index der Synthese z.B. der Unterschied zwischen der Anzahl der Morpheme eines jeden Wortes und dem Mittelwert der Anzahl der Morpheme). Da über die im Material von Greenberg und Cowgill auftretende Streuung keine Nachrichten vorliegen, lässt sich der mittlere Fehler auch nicht nachträglich errechnen. Eine Ausnahme bildet der Index der Agglutination, der als Verhältniszahl zweier Klassenfrequenzen betrachtet werden kann, denn es gibt nur zwei Alternativen: entweder ist die Verbindung agglutinierend oder sie ist es nicht. Dabei kann das Schema des mittleren Fehlers der Verhältniszahl Anwendung finden

$$
\varepsilon(\mathrm{P})=\sqrt{\frac{\mathrm{P}(1-\mathrm{P})}{\mathrm{N}}},
$$

wo $\mathrm{P}$ die Verhältniszahl bzw. der Index der Agglutination ist und $\mathrm{N}$ die Anzahl der Fälle bzw. der Morphemgrenzen im Innern des Wortes. Den Wert von $\mathrm{N}$ entnehmen wir dem Index der Synthese. Da der Index der Synthese M/W die Anzahl der Morpheme im Verhältnis zur Anzahl der Wörter mitteilt und die Anzahl der Wörter bei Greenberg 100 beträgt, ergibt sich für die Anzahl der Morpheme $100 \cdot \mathrm{M} / \mathrm{W}$. Da jedes Wort eine Morphemgrenze weniger besitzt als Morpheme, entspricht in cinem Text von hundert Wörtern die Anzahl der Morphemgrenzen im Wortinnern $(\mathrm{N})$ der Anzahl der Morpheme minus hundert. Es folgt also

$$
\mathrm{N}=100 \cdot \mathrm{M} / \mathrm{W}-100 .
$$

Im folgenden (Tabelle 1) sind die mittleren Fehler und die Vertrauensbereiche der Greenbergschen Indexe der Agglutination auf einer Ebene von $95 \%$ berechnet. Der allgemein üblichen Praxis nach bedeutet das, dass das Ergebnis in den Grenzen $\mathrm{P} \pm 1,96 \cdot \varepsilon \quad 95 \%$ ig zuverlässig ist. 
Tabelle 1

$\begin{array}{lrrrrrr}\text { Sprache } & \text { M/W } & \text { N } & \text { A/J } & \varepsilon & 1,96 \cdot \varepsilon & \begin{array}{c}\text { A/J 95\%ige } \\ \text { Zuverl. }\end{array} \\ \text { Sanskrit } & & & & & & 0,04-0,14 \\ \text { Angelsächsisch } & 2,59 & 159 & 0,09 & 0,023 & 0,045 & 0,042 \\ \text { Persisch } & 112 & 0,11 & 0,030 & 0,058 & 0,05-0,17 \\ \text { Englisch } & 1,52 & 52 & 0,34 & 0,066 & 0,129 & 0,21-0,47 \\ \text { Jakutisch } & 1,68 & 68 & 0,30 & 0,056 & 0,109 & 0,19-0,41 \\ \text { Suaheli } & 2,17 & 117 & 0,51 & 0,046 & 0,091 & 0,42-0,60 \\ \text { Eskimoisch } & 2,55 & 155 & 0,67 & 0,038 & 0,074 & 0,60-0,74 \\ & 3,72 & 272 & 0,03 & 0,010 & 0,020 & 0,01-0,05\end{array}$

Aus der Tabelle geht eindeutig hervor, dass auch bedeutende Unterschiede in den Indexen der Agglutination nicht als zuverlässig gelten können, wenn Texte in einer Länge von hundert Wörtern zugrunde liegen. Unsicher sind die Verhältnisse zwischen dem Sanskrit $(0,09)$, dem Angelsächsischen $(0,11)$ und dem Eskimoischen $(0,03)$. Ferner lässt sich nicht mit Sicherheit sagen, welche Sprache mehr agglutinierend ist, Persisch $(0,34)$ oder Englisch $(0,30)$, Persisch oder Jakutisch $(0,51)$, Jakutisch oder Suaheli $(0,67)$. Sehr wahrscheinlich ist lediglich, dass im Sanskrit, im Angelsächsischen und im Eskimoischen weniger Agglutination begegnet als in den anderen behandelten Sprachen und dass das Suaheli mehr Agglutination aufweist als das Persische und das Englische. Ferner kann es als ziemlich wahrscheinlich gelten, dass es im Jakutischen etwas mehr Agglutination gibt als im Englischen, auch wenn die Wahrscheinlichkeit nicht so deutlich ist wie bei den vorhergehenden Fällen. Mit anderen Worten ergeben die Agglutinationsindexe auf der Basis von hundert Wörtern nichts, was einem Kenner der diesbezüglichen Sprachen nicht ohnehin schon bekannt wäre. Würde man die Vertrauenstufe auf $99 \%$ erhöhen $(\mathrm{P} \pm 2,5758 \cdot \varepsilon)$ oder auf $99,9 \%(\mathrm{P} \pm 3,2905 \cdot \varepsilon)$, wären die Resultate von noch geringerer Signifikanz. Die Bedeutsamkeit der Unterschiede zwischen den Indexen der einzelnen Sprachen kann, wenn man will, mit verschiedenen Methoden genauer getestet werden, z.B. mit Hilfe der Varianzanalyse.

Eine Überprüfung der statistischen Signifikanz der anderen von Greenberg (und Cowgill) errechneten Indexe würde offenbar zu einem ähnlichen Ergebnis führen. Man kann natürlich 
darüber diskutieren, wie genaue Indexwerte überhaupt für nötig befunden werden. Wird aber eine wirklich universale Typologie angestrebt, die im Prinzip vergleichbare Indexe aus allen Sprachen der Welt beinhalten sollte, und will man, dass die zwei Dezimalstellen in den Indexen wirklich signifikant wären, müsste der Wert von $\varepsilon$ kleiner sein als 0,005 . Es hängt von der Streuung ab, wie gross das Material sein muss, damit man auf einen entsprechenden Wert von $\varepsilon$ kommt. Zur Errechnung des Agglutinationsindexes mit diesbezüglicher Genauigkeit erhalten wir den Wert von N nach Schema (3) folgendermassen:

$$
\mathrm{N}=\frac{\mathrm{P}(1-\mathrm{P})}{\varepsilon^{2}}
$$

Der Umfang des Materials hängt also $a b$ von der Grösse des $\mathrm{P}$ bzw. des Agglutinationsindexes. Ist dieser Index niedrig oder hoch, ist weniger Material nötig für ein zufriedenstellendes Ergebnis. Mit wachsendem oder abnehmendem Index steigt auch der Umfang des benötigten Materials, bis er beim Indexwert 0,50 seine oberste Grenze erreicht. Wenn der erforderliche Wert von $\varepsilon$ 0,005 beträgt und der Agglutinationsindex 0,01 oder 0,99 , dann ist $\mathrm{N}=\frac{0,01 \cdot 0,99}{0,005^{2}}=396$. Beträgt der Agglutinationsindex 0,50 ergibt sich für das erforderliche Material die Summe $N=\frac{0,50 \cdot 0,50}{0,005^{2}}=10000$. Damit auch die geringen Unterschiede zwischen den Sprachen signifikant sind, müsste also zur Errechnung eines Agglutinationsindexes in den meisten Fällen ein Text durchgegangen werden, der mehrere tausend Morphemgrenzen im Innern des Stammes enthält. Für eine Berechnung vollständiger Indexreihen mit der verlangten Genauigkeit wären wahrscheinlich Texte in einer Länge von 5 000-10 000 Wörtern nötig.

Die blosse statistische Genauigkeit genügt aber nicht. Auch die morphologische Analyse des Textes müsste so eindeutig sein, dass die Analysen der einzelnen Sprachen untereinander vergleichbar wären. Wie auf den Seiten 208-213 nachgewiesen, 
kann die Greenbergsche Methode in dieser Hinsicht keinen hohen Anforderungen genügen. Die Ungenauigkeit der Forschungsmethode bewirkt, dass auch die Exaktheit von Indexen, errechnet an einem langen Text, nur scheinbar wäre.

Ungeachtet dessen, dass Greenbergs Typologie kritisiert werden kann, dürfte es vorläufig die einzige sein, mit deren Methoden die Sprachen relativ objektiv nach den verschiedenen morphologischen und syntaktischen Kriterien eingeteilt werden können. Ausserdem lassen sich viele ihrer Schwächen zumindest im Prinzip beheben. Die statistische Glaubwürdigkeit kann erhöht werden, indem die Berechnungen an einem ausreichend langen Text vorgenommen werden und indem auch die Repräsentativität des Textes berücksichtigt wird. Auch die grammatische Analyse des Textes kann so entwickelt werden, dass die Anzahl der mehrdeutigen Fälle abnimmt. Das Verfahren ist ferner insofern entwicklungsfähig, dass im Bedarfsfall auch ganz neue Indexe in Anwendung kommen können.

Die generative Grammatik, die in den letzten Jahren in Amerika einen starken Aufschwung genommen hat und vom früheren sog. taxonomischen Strukturalismus weitgehend abweicht, vermag vielleicht auch der Sprachtypologie eine ganz neue Form zu geben. Eine generative Typologie kann natürlich nicht die Prinzipien der Greenbergschen Typologie zum Ausgangspunkt nehmen. Wenn es auch noch verfrüht ist, über die Beschaffenheit einer generativen Typologie Auskunft zu geben, enthält jedoch bereits die augenblickliche Theorie, wie sie von Noam Chomsky, Morris Halle und anderen vertreten wird (Chomsky, Aspects of the Theory of Syntax, Cambridge, Mass. 1965; Chomsky - Halle, The Sound Pattern of English, New York 1968; darin Hinweise auf die übrige Literatur), eine Reihe von Besonderheiten, wodurch die Richtung der generativen Typologie bereits implizit Gestalt annimmt. So wird die morphologische Struktur, insofern es um agglutinierende, fusionierende und symbolische Sprachen geht, durch die phonologische Komponente der generativen Grammatik bestimmt. Ausgangspunkt (input) der phonologischen Komponente ist die Oberflächenstruktur, die zumindest nach der augenblicklichen Theorie stets in dem Sinn "agglutinierend" ist, dass sie sich aus einer Reihe 
lexikalischer und grammatischer Formativa zusammensetzt. Durch die phonologische Komponente erhält diese die phonetische Vertretung. Der Ausgangspunkt der Form der 3. Pl.Prät. lpKo meñne z.B. wäre in der Oberflächenstruktur eine solche Formativkette wie + mănă + Prät. + Pl. + 3. + . Je agglutinierender eine Sprache ist, desto weniger Regeln werden offenbar in der phonologischen Komponente benötigt, und je mehr Fusion und Symbolismus in einer Sprache vorhanden sind, desto komplizierter ist die phonologische Komponente. Die generative Typologie wird also wahrscheinlich eine Typologie von Regeln sein.

Vorläufig bestehen keine Voraussetzungen für typologische Vergleiche auf der Grundlage der generativen Grammatik. Die gesamte Theorie ist noch im Prozess des Werdens und angewandt worden ist sie erst auf einige wenige Sprachen. Wenn also nun die lappischen Dialekte miteinander und mit den anderen finnisch-ugrischen Sprachen verglichen werden sollen, ist beim augenblicklichen Stand der Forschung die Greenbergsche Typologie noch immer das geeignetste Verfahren.

Die Indexe sind aus fünf lappischen Dialekten und fünf anderen finnisch-ugrischen Sprachen errechnet. Von den Greenbergschen Indexen wurden die drei letzten für die Syntax als für unser Thema nicht wesentlich weggelassen. Dafür wurden zwei neue Indexe eingeführt. Wie oben (S. 207) festgestellt, macht Greenberg keinen Unterschied zwischen fusionaler und symbolischer Methode. Da dieser Unterschied meines Erachtens jedoch typologisch wichtig ist, drücken im folgenden zwei Indexe den Grad des Symbolismus aus. Das eine ist der Index der affixalen Derivation $\mathrm{AxD} / \mathrm{D}$, wo $\mathrm{AxD}$ die Anzahl der affixalen Ableitungsmorpheme ausmacht, und das andere der Index der affixalen Inflexion AxI/I, wo AxI die Anzahl der affixalen Inflexionsmorpheme angibt. Die obere Grenze dieser Indexe ist 1, wenn nämlich alle Derivations- bzw. Inflexionsmorpheme durch Affixe ausgedrückt sind und kein Symbolismus auftritt. Je niedrigere Werte die Indexe aufzeigen, umso mehr Symbolismus hat die Sprache. Der Umfang des Symbolismus kommt in diesen Indexen also gewissermassen negativ zum Ausdruck. Die fusionale Methode zeigt sich darin, dass der Agglutinationsin- 
dex niedrig ist und die Indexe der affixalen Derivation und der affixalen Inflexion hoch sind.

Die Indexe der lappischen Dialekte sind errechnet worden an Texten von 300 Wörtern. Unter Berücksichtigung der Fehlerquellen in der Analyse, die eine grössere statistische Genauigkeit mit einem umfangreicheren Material teilweise zunichte machen (s. S. 217), wurden keine längeren Texte behandelt. Wie aus den Tabellen ersichtlich ist, erhält man bereits mit einem Text von 300 Wörtern eine Genauigkeit, die deutlich die Tendenzen der einzelnen Dialekte zu zeigen vermag.

Die fünf übrigen finnisch-ugrischen Sprachen dienen lediglich als Vergleiche. Da in erster Linie nur die typologischen Besonderheiten aufgedeckt werden sollen, in denen das Lappische eindeutig abweicht vom allgemeinen finnisch-ugrischen Typ, konnten wir uns mit Texten begnügen, die nur 100 Wörter umfassen. Eine detaillierte komparative Untersuchung der finnisch-ugrischen Sprachen würde natürlich ein wesentlich umfangreicheres Material beanspruchen, doch müssten zunächst auch noch einige Probleme der deskriptiven Grammatik für viele Sprachen geklärt werden, damit die den Indexen zugrunde liegende grammatische Analyse genau würde.

Als Material wurden die folgenden Texte verwendet:

LpSd. Knut Bergsland, Røros-lappisk grammatikk (Oslo 1946), S. 251-255, Dan jijjan gâlg am usw.

LpN Konrad Nielsen, Lærebok i lappisk, II, Tekster (Oslo 1926), S. 117, Bâggjë-sabmèlâžžâak âdnik usw. 100 Wörter, S. 153, Guok'tě ol'bma gāwnâdik usw. 100 Wörter; J. Qvigstad, Lappiske eventyr og sagn I (Oslo 1927), S. 2, Vuorâcâas rakkâải base usw. 100 Wörter.

LpI Sabmelaš 1968 n:0 1-2, S. 8, Lavrukkäš uusai udda joavhu usw.

LpSk. T. I. Itkonen, Koltan- ja kuolanlappalaisia satuja

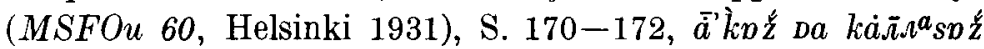
jiẹ̌lle usw. 200 Wörter, S. 176, kuов́ž́a ii vuaite kop̄ped usw. 100 Wörter.

LpKld. a.a.O. $246-249$, rièmje lei usw.

Fi. Pentti Haanpää, Noitaympyrä (Helsinki 1964, 1. Aufl. 1956), S. 5, Tapasin kaupungin kadulla usw. 
MordE H. Paasonen, Mordwinische Chrestomathie (Hilfsmittel für das Studium der finnisch-ugrischen Sprachen IV, 2. Aufl. Turku 1953), S. 11, śup̀av eŕźan ulneś usw.

Tšer. Yrjö Wichmann, Tscheremissische Texte (Hilfsmittel für das Studium der finnisch-ugrischen Sprachen V, 2. Aufl.,

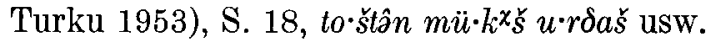

Wotj. Yrjö Wichmann, Wotjakische Chrestomathie (Hilfsmittel für das Studium der finnisch-ugrischen Sprachen II, 2. Aufl., Turku 1954), S. 7-8, odig jegit pi usw.

Ung. István Nyirkos, Unkarin lukemisto sanastoineen (Helsinki 1965), S. 84 (Zsigmond Móricz: Hét krajcár), Jól rendelték azt az istenek usw.

Bei der Analyse sowie bei der Errechnung der Indexe wurden die Greenbergschen Grundprinzipien möglichst genau befolgt, bis auf die oben genannten Einschränkungen. Die Agglutination unterlag in meiner Analyse jedoch offenbar einer strengeren Auswahl, denn die von mir errechneten Agglutinationsindexe liegen durchweg niedriger verglichen mit denen von Greenberg für die einzelnen Sprachen. Für alle Indexe wurde unter Verwendung von Schema (1) und (2) der mittlere Fehler errechnet, von da aus dann wiederum der Vertrauensbereich auf einer Ebene von $95 \%$. (Index $\pm 1,96 \cdot \varepsilon$ ).

Die Tabelle 2 lässt sofort folgende Beobachtungen zu:

1. Bedeutende Differenzen zwischen den lappischen Dialekten zeigen sich lediglich bei den Indexen der Suffixe (S/W) und der affixalen Inflexion ( $\mathrm{AxI} / \mathrm{I})$; die übrigen Indexe weisen keine wesentlichen interdialektalen Unterschiede auf.

2. Das Norwegisch-, Skolt- und Kildinlappische bilden eine Gruppe, innerhalb derer die Indexwerte überhaupt kaum voneinander abweichen. Von dieser Gruppe hebt sich stark das Südlappische ab und in geringerem Umfang das Inarilappische.

Betrachten wir nun die Indexwerte für die Dialekte einzeln.

Der Index der Synthese (M/W) schwankt von 1,85 (lpKld.) bis 2,09 (lpSd.). Die Variation ist nicht sehr gross. Nur im Südlappischen ist der Index eindeutig höher als anderswo, so dass er beachtlich abweicht von denen des Norwegisch- und Kildinlappischen. Nach Greenberg hat eine Sprache mit einem Synthese-Index unter 2,00 als analytisch zu gelten, während die 
Tabelle 2

\begin{tabular}{|c|c|c|c|c|c|c|}
\hline Sprache & $\mathrm{M} / \mathrm{W}$ & $\pm 1,96 \cdot \varepsilon$ & $\mathbf{A} / \mathbf{J}$ & $\pm 1,96 \cdot \varepsilon$ & $\mathrm{R} / \mathrm{W}$ & $\pm 1,96 \cdot \varepsilon$ \\
\hline LpSd. & 2,09 & $1,99-2,19$ & 0,01 & $0,00-0,02$ & 1,02 & $1,00-1,04$ \\
\hline $\mathrm{N}$ & 1,89 & $1,80-1,98$ & 0,01 & $0,00-0,02$ & 1,02 & $1,00-1,04$ \\
\hline I & 1,96 & $1,87-2,05$ & 0,03 & $0,01-0,05$ & 1,03 & $1,01-1,05$ \\
\hline Sk. & 1,93 & $1,84-2,02$ & 0,01 & $0,00-0,02$ & 1,02 & $1,01-1,03$ \\
\hline Kld. & 1,85 & $1,77-1,93$ & 0,01 & $0,00-0,02$ & 1,01 & $1,00-1,02$ \\
\hline $\mathrm{Fi}$ & 2,22 & $2,01-2,43$ & 0,25 & $0,17-0,33$ & 1,07 & $1,01-1,13$ \\
\hline MordE & 1,81 & $1,67-1,95$ & 0,20 & $0,11-0,29$ & 1,00 & 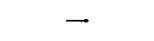 \\
\hline Tšer. & 2,05 & $1,88-2,22$ & 0,33 & $0,24-0,42$ & 1,04 & $1,00-1,09$ \\
\hline Wotj. & 2,12 & $1,94-2,30$ & 0,35 & $0,26-0,44$ & 1,13 & $1,06-1,20$ \\
\hline \multirow[t]{2}{*}{ Ung. } & 1,91 & $1,69-2,13$ & 0,37 & $0,27-0,47$ & 1,07 & $1,01-1,13$ \\
\hline & \multicolumn{2}{|l|}{$\mathrm{D} / \mathrm{W}$} & \multicolumn{2}{|l|}{$\mathrm{I} / \mathrm{W}$} & \multicolumn{2}{|l|}{$\mathrm{S} / \mathrm{W}^{1}$} \\
\hline LpSd. & 0,28 & $0,22-0,34$ & 0,80 & $0,72-0,88$ & 1,03 & $0,93-1,13$ \\
\hline $\mathrm{N}$ & 0,20 & $0,14-0,26$ & 0,63 & $0,57-0,69$ & 0,67 & $0,59-0,75$ \\
\hline I & 0,30 & $0,23-0,37$ & 0,61 & $0,54-0,68$ & 0,76 & $0,68-0,84$ \\
\hline Sk. & 0,18 & $0,13-0,23$ & 0,74 & $0,67-0,81$ & 0,67 & $0,59-0,75$ \\
\hline Kld. & 0,19 & $0,14-0,24$ & 0,64 & $0,58-0,70$ & 0,59 & $0,51-0,67$ \\
\hline $\mathrm{Fi}$ & 0,39 & $0,24-0,54$ & 0,69 & $0,56-0,82$ & 1,13 & $0,93-1,33$ \\
\hline MordE & 0,10 & $0,03-0,17$ & 0,71 & $0,59-0,83$ & 0,80 & $0,66-0,94$ \\
\hline Tšer. & 0,17 & $0,09-0,25$ & 0,75 & $0,63-0,87$ & 1,01 & $0,85-1,17$ \\
\hline Wotj. & 0,13 & $0,05-0,21$ & 0,84 & $0,69-0,99$ & 0,98 & $0,81-1,15$ \\
\hline \multirow[t]{2}{*}{ Ung. } & 0,24 & $0,14-0,34$ & 0,60 & $0,44-0,76$ & 0,82 & $0,62-1,02$ \\
\hline & \multicolumn{2}{|c|}{$\mathrm{AxD} / \mathrm{D}$} & \multicolumn{2}{|c|}{$A x I / I$} & & \\
\hline LpSd. & 0,98 & $0,95-1,00$ & 0,94 & $0,91-0,97$ & & \\
\hline $\mathrm{N}$ & 0,95 & $0,89-1,00$ & 0,70 & $0,63-0,77$ & & \\
\hline I & 0,91 & $0,85-0,97$ & 0,78 & $0,72-0,84$ & & \\
\hline Sk. & 1,00 & - & 0,67 & $0,61-0,73$ & & \\
\hline Kld. & 1,00 & - & 0,62 & $0,55-0,69$ & & \\
\hline $\mathrm{Fi}$ & 1,00 & - & 0,99 & $0,96-1,00$ & & \\
\hline MordE & 1,00 & - & 0,99 & $0,96-1,00$ & & \\
\hline Tšer. & 1,00 & - & 1,00 & - & & \\
\hline Wotj. & 1,00 & - & 0,99 & $0,97-1,00$ & & \\
\hline Ung. & 1,00 & - & 0,97 & $0,91-1,00$ & & \\
\hline
\end{tabular}

1 Für das Ungarische wurde statt des Indexes der Suffixe (S/W) der Index der Affixe (Ax/W) errechnet, wozu neben den Suffixen auch die Präfixe gehören ( $A x=A n z a h l$ der Affixe). Die Textprobe enthielt zwei Prăfixe. 
Werte 2,00-3,00 typisch seien für synthetische Sprachen ( $I J A L 26$ S. 194). Nach unseren Resultaten überschreitet von den lappischen Dialekten nur das Südlappische die Grenze von 2,00. Die Synthese der lappischen Dialekte entspricht dem Mittelwert der Indexe 1,94 nach zu schliessen - auf ieur. Seite z.B. der des Bengalischen $(1,90)$ und Hethitischen $(1,95)$, ist eindeutig höher als im modernen Persisch $(1,52)$ und modernen Englisch $(1,68)$ und niedriger als im Sanskrit $(2,56,2,59)$, im Asoka $(2,52)$ und im Griechisch des Neuen Testaments $(2,45)$ (Greenberg a.a.0. 193, Cowgill Universals of Language S. 99).

Die Grösse des Index für Synthese beim Südlappischen ist eine Folge des Reichtums an Derivations- und Inflexionsmorphemen. Wie aus der Tabelle ersichtlich ist, hat das Süd- und Inarilappische einen höheren Derivationsindex als es in den anderen Dialekten der Fall ist. Als wichtig hat vielleicht die Differenz zwischen lpI $(0,30)$ und lpSk. $(0,18)$ zu gelten. Der Index der Inflexion wiederum ist im Südlappischen $(0,80)$ beträchtlich höher als im Norwegischlappischen $(0,63)$, im Inarilappischen $(0,61)$ und Kildinlappischen $(0,64)$. Die auf die Indexe der Synthese und der Inflexion einwirkende Anzahl der Inflexionsmorpheme wird im Südlappischen vielleicht am deutlichsten durch die beiden folgenden Faktoren erhöht:

1. Das im Präteritum auftretende Morphem $a$ der $3 . \mathrm{Sg}$. (algiestäjj-a 'beginnen', vgl. 3. Sg.Prs. boåt-a 'kommen'), das in den übrigen untersuchten Dialekten nicht begegnet.

2. Die Aufteilung der Inessiv- und Elativendungen in zwei Morpheme aufgrund folgender Matrizen: In.Sg. dä-š-ńie : El.Sg.

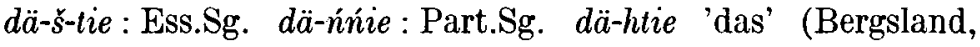
Røros-lappisk grammatikk S. 93, 104; über Matrix als morphologisches Kriterium s. Greenberg a.a.O. 188-189). In den übrigen untersuchten Dialekten entspricht dem südlappischen Inessiv und Elativ ein Kasus, der Lokativ (lpN Sg. -st, Pl. -n), und eine Matrix, die zur Aufteilung der Endung berechtigen würde, lässt sich nicht konstruieren.

Die Gesamtwirkung der genannten Faktoren im analysierten Text beträgt 0,06. Entspräche das Südlappische in dieser Hinsicht den übrigen Dialekten, erhielten wir als Index der Synthese 2,03 und als Index der Inflexion 0,74 , wobei dann ledig- 
lich die Differenz zwischen den Synthese-Indexen im Süd- und im Kildinlappischen vielleicht als signifikant betrachtet werden könnte.

Der Agglutinationsindex $(\mathrm{A} / \mathrm{J})$ ist in allen lappischen Dialekten ausserordentlich niedrig, weit unter dem Wert für irgendeine von Greenberg und Cowgill untersuchte Sprache. Dem Lappischen in dieser Hinsicht am nächsten steht das Eskimoische mit einem Agglutinationsindex von 0,03 (Greenberg a.a.O. 193). Die Differenzen zwischen den Indexwerten der lappischen Dialekte sind statistisch nicht relevant. In allen lappischen Dialekten ist der nichtautomatische Wechsel von Morphemen so allgemein, dass sehr wenig Agglutinationsfälle übrig bleiben, falls die Forderung nach dem automatischen Wechsel so genau genommen wird wie es in der vorliegenden Untersuchung durchgehend geschieht. Nichtautomatischer Wechsel trifft für allem zu für Nominal- und Verbalstämme, für die meisten Deklinations- und Konjugationsmorpheme und viele Ableitungssilben. Es ist selten, dass zwei nichtwechselnde Morpheme nebeneinander stehen. In den analysierten Texten fanden sich folgende typische Agglutinationsfälle: lpSd. da-kka 'hierher', $-s-n$ Inessivendung, -s-t'a Elativendung, $\mathrm{lpN} d \hat{a}-d_{e}$ 'umso, je', $d a-l$ 'jetzt', lpI to-bbee-n 'dort', lpSk. to-škka 'dorthin'.

Auch die Indexe für Komposita $(\mathrm{R} / \mathrm{W})$ weisen keine besonderen Unterschiede zwischen den Dialekten auf.

Am interessantesten für unsere Untersuchung sind die Indexe der Suffixe (S/W), der affixalen Derivation $(\mathrm{AxD} / \mathrm{D})$ und der affixalen Inflexion (AxI/I), wodurch die lappischen Dialekte tatsächlich in typologisch verschiedene Gruppen geteilt werden. Die Differenzen in der Formmethode zeichnen sich ebenfalls durch diese Indexe am deutlichsten ab. Die Zahlen zeigen, dass das Südlappische den grössten Teil seiner Derivation und seiner Inflexion durch Suffixe erledigt, während die anderen Dialekte für die Derivation hauptsächlich Suffixe verwenden, für die Inflexion aber neben den Suffixen in beachtlichem Masse auch den Symbolismus. Von den Unterschieden zwischen den Indexen der affixalen Derivation kann lediglich die Differenz zwischen dem Inarilappischen $(0,91)$ und dem Skolt- und Kildinlappischen $(1,00)$ als statistisch signifikant 
betrachtet werden. Der relativ niedrige Wert des Inarilappischen ist darauf zurückzuführen, dass im Text zweimal Nom.Pl. sämiliih, zweimal Gen.Pl. sämilij und einmal Akk.Pl. sämilijd begegnet, vom Wort sämilaž 'Lappe'. In diesem Wort treten zwei Ableitungsmorpheme auf, im Nom.Sg. $l$ und $\check{z}$, von denen das letztere in den oben genannten Kontraktionsformen symbolisch realisiert wird, vgl. z.B. Nom.Pl. immeeleh (vom Wort immeel 'Gott'), wo nur das Ableitungsmorphem $l$ steht und wo in der dritten Silbe der Nom.Pl.-Form der Vokal $e$ dem $i i$ der -lă̌-Ableitung entspricht. Offenbar würde auch ein umfangreicheres Material für das Inarilappische einen niedrigeren Index der affixalen Derivation ergeben als für das RussischLappische, denn ausser den genannten Kontraktionsformen der Deminutivableitungen besitzt das Inarilappische bedeutend mehr Kontraktionsformen von $j e$-Verben als das RussischLappische (s. MSFOu 143 S. 81-95); in all diesen Kontraktionsformen ist das Ableitungselement symbolisch vertreten.

Im Hinblick auf die Flexionsmethode ist das Südlappische am wenigsten symbolisch; der Anteil des Symbolismus wächst in östlicher Richtung. Diese Tendenz zeigt sich an den Indexwerten der affixalen Inflexion, auch wenn die Unterschiede zwischen dem Norwegisch- $(0,70)$, Skolt- $(0,67)$ und Kildinlappischen $(0,62)$ zu klein sind, um im rein statistischen Sinne Beweiskraft zu haben. Auffallend ist das Inarilappische, dessen Index $(0,78)$ beachtlich über dem des Kildinlappischen liegt, und eindeutig höher - wenn auch nicht statistisch relevant ist als im Norwegisch- und Skoltlappischen.

Die Tabellen zeigen, dass keiner der untersuchten lappischen Dialekte agglutinierend ist. Das Südlappische hat als fusionierend zu gelten: es verwendet hauptsächlich Suffixe, die Morpheme aber verbinden sich normalerweise nicht mechanisch miteinander. Die übrigen Dialekte könnten als fusionierendsymbolisch gekennzeichnet werden.

Vergleicht man die Ergebnisse aus den lappischen Dialekten mit den Indexen der fünf anderen finnisch-ugrischen Sprachen, stellt man folgendes fest:

1. Hinsichtlich des Indexes der Synthese ist das gesamte untersuchte Material relativ homogen. Die Werte liegen auf beiden Seiten von 2,00 zwischen 1,81 (mordE) und 2,22 (fi). 
2. Der Agglutinationsindex ist im Lappischen beträchtlich niedriger als in den fünf anderen finnisch-ugrischen Sprachen. Wie auf S. 220 bereits angemerkt, ist der Begriff der Agglutination hier offenbar enger gefasst (strenger ausgelegt) als bei Greenberg, da die Indexwerte in allen finnisch-ugrischen Sprachen derart niedrig liegen. Nach Greenberg kann eine Sprache als agglutinierend bezeichnet werden, wenn ihr Agglutinationsindex über 0,50 liegt. In keiner der hier untersuchten finnischugrischen Sprachen wird dieser Wert erreicht, obgleich u.a. das Wotjakische, das Tscheremissische und das Ungarische mit geprüft wurden, die normalerweise als agglutinierend gelten.

3. Der Index der Komposita liegt im Mordwinischen $(1,00)$ und Tscheremissischen $(1,04)$ in der Nähe der Werte für die lappischen Dialekte, im Finnischen und Ungarischen etwas jedoch nicht wesentlich - höher $(1,07)$ und im Wotjakischen eindeutig höher $(1,13)$ als im Lappischen.

4. Die Indexe für Derivation in den lappischen Dialekten, 0,18 (lpSk.) - 0,30 (lpI), liegen innerhalb der Grenzwerte der untersuchten verwandten Sprachen 0,10 (mordE) und 0,39 (fi). Das Inari- und das Südlappische stehen dem Finnischen und dem Ungarischen $(0,24)$ relativ nahe, während die übrigen Dialekte (lpN 0,20, Kld. 0,19 und Sk. 0,18) zwischen dem Ungarischen und dem Tscheremissischen $(0,17)$ bleiben.

5. Der Index der Inflexion variiert recht wenig: 0,60 (ung.) 0,84 (wotj.). Im Hinblick auf den Reichtum an Inflexion bilden die finnisch-ugrischen Sprachen eine ziemlich einheitliche Gruppe. Die Schwankung innerhalb der lappischen Dialekte, 0,61 (lpI) - 0,80 (lpSd.) ist fast ebenso gross wie in der gesamten untersuchten Sprachgruppe.

6. Die Differenz zwischen dem niedrigsten und dem höchsten Index der Suffixe (Affixe) ist dagegen beachtlich gross: 0,59 (lpKld.) - 1,13 (fi). Von Einfluss sind hier vor allem die norwegisch- und ostlappischen Dialekte. Lässt man sie ausser acht, liegt der niedrigste Wert bei 0,80 (mordE). Mit Ausnahme des Südlappischen haben alle anderen lappischen Dialekte einen niedrigeren Wert; das Südlappische folgt im Reichtum an Suffixen gleich nach dem Finnischen $(1,13)$ an zweiter Stelle.

7. Mit dem oben Ausgeführten hängt eng zusammen, dass jene vier lappischen Dialekte, die einen niedrigen Suffix-Index ha- 
ben, auch einen niedrigen Index der affixalen Inflexion besitzen (lpI 0,78-lpKld. 0,62). Das Südlappische schliesst sich auch hier an die anderen finnisch-ugrischen Sprachen mit einem hohen Index der affixalen Inflexion an $(0,97-1,00)$. Derivationssymbolismus war in den Proben der fünf finnisch-ugrischen Sprachen überhaupt nicht enthalten $(\mathrm{AxD} / \mathrm{D}=1,00)$, während Spuren davon im Süd-, Norwegisch- und Inarilappischen nachweisbar sind $(0,91-0,98)$.

Im Hinblick auf ihre morphologische Methode bilden die fünf verwandten Sprachen eine typologisch recht einheitliche Gruppe. Die sichtbarste Abweichung liegt wohl in der Verwendung der Präfixe im Ungarischen. Wie die Indexe zeigen, sind diese Sprachen ziemlich synthetisch und fusionierend-agglutinierend. Diesem finnisch-ugrischen Typ steht von den lappischen Dialekten das Südlappische am nächsten, das sich jedoch durch ein fast vollständiges Fehlen von Agglutination abhebt. Die übrigen lappischen Dialekte weichen von den untersuchten finnisch-ugrischen Sprachen wieder mehr ab. "Nicht-finnischugrische" Züge, die sie aufweisen, sind ausser der geringen Agglutination der Symbolismus in der Inflexion und im Hinblick auf die Synthese der seltene Gebrauch von Affixen.

\section{DIE FORMMETHODE DES LAPPISCHEN}

\subsection{Der geringe Anteil der Agglutination}

Historisch gesehen geht das Fehlen der Agglutination im Lappischen auf zwei ganz verschiedene Erscheinungen zurück. Einerseits begegnet ein Wechsel von Allomorphen, der heute nichtautomatisch ist, früher aber völlig automatisch und also agglutinierend war, z.B. ist der Wechsel des Präteritumcharakter in $\operatorname{lpN}$ (1. Sg. goč'čum - 3. Sg. goččoi 'rufen, einladen') zurückzuführen auf den Wechsel urlp. ${ }^{*} j \sim{ }^{*} j e$, der folgendermassen automatisch war: wenn der Präteritumcharakter im Wortauslaut stand oder wenn ihm ein Suffix vom Typ ${ }^{*} x_{3}(x)$ folgte, trat $* j$ als Charakter auf, folgte ihm aber ein Suffix vom Typ ${ }^{*} x$, lautete der Charakter $* j \ddot{e}$. Dieser Wechsel der Charaktervarianten gehört an sich zu einer umfassenderen und älteren 
Eigenschaft der finnisch-ugrischen Sprachen, zum Fehlen von Konsonantenverbindungen im Wortauslaut nämlich (s. E. Itkonen, Suomalais-ugrilaisen kielen- ja historiantutkimuksen alalta S. 68). Dass aus dem automatischen Wechselverhältnis

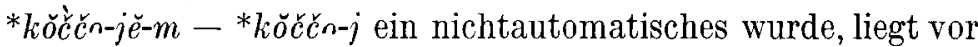
allem an der Kontraktion $\left({ }^{*} n j \check{e}>\right)^{*} \breve{u} j \check{e}>u$. Andererseits treten aber zum selben Morphem gehörende Allomorphe auf, deren Verhältnis nicht lautgeschichtlich erklärt werden kann. Solche Allomorphe vertreten meistens verschiedene Morpheme, die erst im Urlappischen, in einigen Fällen eventuell auch später, in komplementäre Distribution gelangten. Ein typisches Beispiel ist der Akkusativ, der im Singular auf $-m$ endet oder auf eine solche Endung zurückgeht ( $1 \mathrm{pN} d \hat{a} m$ vom Pronomen dât 'das', guole $<{ }^{*}$ kolem vom Wort guolle 'Fisch', IpSd. goåtiem vom Wort goåtie 'Hütte, Zelt' usw.), im Plural aber auf -d, -də usw. auslautet (lpN dâid, güliid, Sd. goåt'ajd’a). Von den Allomorphen vertritt $m$ die uralische Akkusativendung ${ }^{*} m$, während $d$, do usw. für die uralische Ablativendung ${ }^{*} t a{ }^{*} t \ddot{a}$ stehen. Der Ablativ trat offenbar schon im Frühurfinnischen als Kasus des partiellen Objekts auf, welche Praxis den Anstoss hat geben können für den im Urlappischen erfolgten Übergang zur Funktion des pluralischen Akkusativs (Ravila FUF 23 S. 54-55). Nur in seltenen Fällen waren die Vorgänger der nichtautomatisch wechselnden Allomorphe schon vor der urlappischen Zeit nichtautomatische Allomorphe desselben Morphems. Hierher gehört z.B. der Pluralcharakter der lappischen Deklination, im Nominativ $-k$ oder dessen Vertreter und in den übrigen Kasus $-i$ - oder dessen Vertreter. Der Wechsel lp $k \sim i$ entspricht dem osfi. $t \sim i, j$ und geht zurück auf den frühurfi. Wechsel $t \sim j$, z.B. lpN Nom.Pl. guolek - Akk.Pl. gūliid, fi. Nom.Pl. kalatPart.Pl. kaloja $<$ frühurfi. ${ }^{*} k a l a-t-{ }^{*} k a l a-j$-ta. Wenigstens bis ins Frühurfi. geht ebenfalls jene im Schwedischlappischen erhaltene Eigenschaft der Konjugation zurück, dass der Präteritumcharakter des Verneinungsverbs ein anderer ist als bei den übrigen Verben, z.B. IpSd. 1. Sg.Prät. Verneinungsverb $e-\check{c} \check{c} \partial-m, 2 . e-\check{c} \check{c}-h, 3 . e-\check{c} \check{c} \partial j$ usw., lpL $i-d t j i-v, i-d t j i-h, i-t t j \bar{\imath}$ usw.,

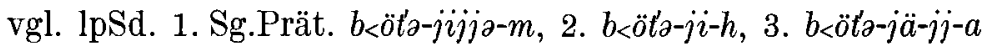

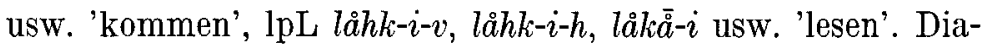


lektweise hat das Verneinungsverb auch im Estnischen und Livischen einen eigenen Präteritumcharakter, z.B. estn. $e-s i-n$, $e-s i-t, e-s$, liv. $i-z$. In der einen oder anderen Form ist diese Zweiteilung offenbar auch noch älter als das Frühurfinnische (s. MSFOu 143 S. 211-213).

Der Reichtum an Allomorphen, verursacht durch den Synkretismus von Morphemen verschiedener Herkunft, ist eine für das Lappische typische Erscheinung. In der Deklination des Späturlappischen hatten zumindest der Genitiv, der Akkusativ, der Illativ und der Komitativ im Plural andere Endungen als im Singular. Dialektweise ging die Entwicklung weiter, und so sind z.B. im gegenwärtigen Norwegischlappisch alle Kasusendungen mit Ausnahme von Abessiv und Essiv im Singular und Plural unterschiedlicher Herkunft. Nach Erkki Itkonen sollte durch diese Verschiedenheit die Opposition von Singular und Plural betont werden (FUF 37 S. 133). In der Konjugation kommt die Heterogenität der urlp. Morpheme in vielfacher Hinsicht zum Ausdruck. Das $m$-Element der 1. P. begegnet im Sg. und im Du. und Pl. des Prät. und Kond. (1. Sg. Prs. *měnĕm, 1. Sg.Prät. *měñèjěm, 1. Du. *měnějměn, 1. Pl. *měnĕjmek > lpN mânâm, mânnim, mânâime, mânâimek usw. 'gehen'). Im Prs., Pot. und Imp. lautete die Endung der 1. Du.

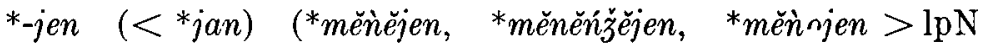
mânni, mânâžed'ni pleon., mân'no) und die der 1. Pl. *-p̀e,

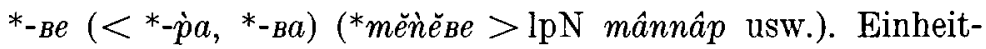
licher ist die 2. P. Überall ausser im Sg. Imp. dürfte das Element ${ }^{*} t,{ }^{*}$ aufgetreten sein. Die 3 . P. wurde durch die folgenden Elemente charakterisiert: im Sg. des Prs., Prät., Pot. und Kond., im Du. und Pl. des Prät. und Kond. durch $\emptyset$ (3. Sg. Prs. *mĕña, 3. Sg. Prät. *mĕnĕj, 3. Pl. *mĕñĕjĕn >lpN mânna, mânâi, mânni usw.), im Du. des Prs. und Pot. durch ${ }_{-B} a-$ (3. Du.Prs. *mĕñĕвan $>\operatorname{lpN}$ mânnâbâ), im Pl. des Prs. und Pot. durch *-je- $\left(<^{*}-j a^{-}\right)$(3. Pl. Prs. ${ }^{*}$ mĕñĕjet $>\operatorname{lpN}$ mânnik)

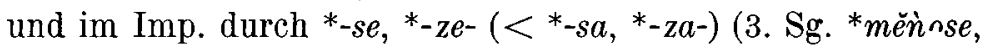
3. Du. *mĕǹnzen, 3. Pl. *mĕǹnzek $>\operatorname{lpN}$ mânnus, mânnuskâ anal., mânnusek).

Im Präsens kommt der nichtagglutinierende Charakter auch in anderer Art und Weise zum Ausdruck. Die in den dua- 
lischen und pluralischen Formen auftretenden Vertreter der Elemente ${ }^{*} j a$ und ${ }^{*} p a$ sind nur für das Präsens charakteristisch. Sie können also als präsentische Kennzeichen gelten. Lediglich in dieser Funktion begegnen sie aber nur in der 2. P.Du. und Pl., wo der eigenen Personalendung der Charakter ${ }^{*}{ }_{B \varepsilon} \sim{ }^{*} \grave{p} e$ folgt (2. Du. *mĕǹĕscteden, 2. Pl. *mĕñĕsciedek $>\operatorname{lpN} m a \hat{n}$ nâbat'te, mânnâbêttit). In den übrigen Formen hatten die Vertreter der Suffixe ${ }^{*} j a$ und *pa auch die Funktion der Personalendung, obwohl sie nicht ausschliesslich eine genügende Information über die betreffende Person enthielten; erst die Kenntnis dessen, ob und welcher Numeruscharakter daran tritt, schaffte Sicherheit über die Person. Das Element *je trat sowohl in der 1. als auch in der 3. P. auf. Trat das Dualzeichen $n$ daran, handelte es sich um die 1. P. Du., verband sich aber das Pluralzeichen $t$ damit, entstand die 3. P.Pl. Ohne Numeruscharakter kennzeichnete ${ }^{*} \dot{p} e,{ }_{B}{ }_{B}$ die 1. P.Pl., wenn dagegen der Dualcharakter an das Suffix $*_{B} a$ gleichen Ursprungs trat, war die Form der 3. P.Du. entstanden. Die Struktur der diesbezüglichen Formen könnte so ausgelegt werden, dass Tempus- und Numeruscharakter zusammen implizit auch die Person ausdrückten. Näher dazu s. MSFOu 143 S. 350-354, E. Itkonen $F U F 37$ s. $98-117$.

Charakteristisch für das Urlappische war also ein Reichtum an nichtautomatischen Allomorphen sowohl in der Deklination als auch in der Konjugation. Da einerseits im Frühurlappischen kein nichtautomatischer Lautwechsel nachgewiesen werden konnte und er auch im Späturlappischen nur in relativ geringem Umfang auftrat, kann man feststellen, dass die AllomorphVerbindungen trotz des im Urlappischen häufigen nichtautomatischen Wechsels von Allomorphen vom phonologischen Standpunkt aus gesehen vor allem in früherer Zeit fast vollständig automatisch waren. Das Urlappische ist also keine agglutinierende Sprache gewesen. Die Morphologie des Urlappischen wird vielleicht am besten durch Sapirs Terminus der "regelmässigen Fusion» (s.S. 206) wiedergegeben. Es lässt sich schwer sagen, wie weit diese urlappische Methode zurückreicht; unter Berücksichtigung der Tatsache, dass es im Osfi., Mordw. und Tscher. nicht so viel Wechsel von Allomorphen verschie- 
dener Herkunft gibt wie im Lappischen, scheint es wahrscheinlich zu sein, dass die finnisch-wolgaische Ursprache und das Frühurfinnische agglutinierender waren als das Urlappische und dass die Entwicklung der lappischen morphologischen Methode zu einer regelmässigen Fusion erst im Urlappischen eingesetzt hat.

Unregelmässige Fusion und Symbolismus, wie sie vor allem in der Distinktivität der Wechsel im Innern des Stammes zum Ausdruck kommen, sind die Folgeerscheinung einer Entwicklung, die erst in der Schlussphase des Urlappischen begann und in den einzelnen Dialekten dann verschieden weiterging; die Agglutination ist dadurch aus dem Lappischen fast völlig vurschwunden. In den folgenden Abschnitten beschäftigen wir uns mit jenem Fehlen der Agglutination, das auf einem Distinktivwerden der Wechsel im Innern des Stammes beruht.

\section{2. Über paradigmatische Lautwechsel im Stamminnern}

Die Formen der paradigmatischen Lautwechsel im Stamminnern, die die lappische Morphologie verwendet, lassen sich folgendermassen gruppieren:

1. Stufenwechsel und damit verbundener qualitativer und quantitativer Wechsel (Abkürzung $\mathrm{S}$ ).

2. Durch Überdehnung verursachter Wechsel (E).

3. Strukturtypenwechsel (T).

4. Metaphoniewechsel (M):

- in der betonten Silbe (M1)

- in der unbetonten Silbe (M2).

5. Qualitativer Wechsel durch Kontraktion (C).

6. Wechsel durch Vokalverschmelzung (F).

7. Sonstige qualitative Wechsel (A).

Um im folgenden die Stellung des Wechsels oder des Lautwandels im Wort kurz definieren zu können, verwende ich die folgenden Bezeichnungen für die Teile des Wortes:

- Anlautkonsonant oder -konsonantismus = anlautender Konsonant oder Konsonantenverbindung, 
- Inlautvokal(ismus) $=$ Vokal(ismus) der 1 . Silbe eines 2und 3-silbigen Stammes sowie der 3 . Silbe eines 4- und 5-silbigen Stammes,

- Inlautkonsonantismus $=$ Konsonantismus an der Grenze zwischen 1. und 2. Silbe bei einem 2- und 3-silbigen Stamm sowie zwischen 3 . und 4. Silbe bei einem 4- und 5-silbigen Stamm,

- Nebenvokal(ismus) $=$ Vokal(ismus) der 2. (4.) Silbe eines 3-(5-) silbigen Stammes,

- Nebenkonsonantismus $=$ Konsonantismus an der Grenze zwischen 2. und 3. (4. und 5.) Silbe bei einem 3- (5-) silbigen Stamm,

- Grenzvokal(ismus) = Auslautvokal des Stammes oder dessen Stelle einnehmender suffixaler Vokal(ismus),

- Suffix(e) = die Flexionssuffixe ausser dem Grenzvokal(ismus).

Z.B.

$\begin{array}{cccc}\text { Anlaut- } & \begin{array}{c}\text { Inlaut- } \\ \text { vons. }\end{array} & \begin{array}{c}\text { Inlaut- } \\ \text { kons. }\end{array} & \begin{array}{c}\text { Grenz- } \\ \text { vok. }\end{array}\end{array}$

$\begin{array}{ccccc}g & -i e & -l^{\prime} d & -e & -t \\ g & -i & -l d & -i & -i-m e-k \\ g & -i & -l^{\prime} d & -u & -s \\ m\left(u i^{\prime} t a \hat{l}\right) & -\hat{a} & -d^{\prime} d & -\hat{a} & -t\end{array}$

$\begin{array}{ccccc}\text { Anlaut- Inlaut- } & \text { Inlaut- } & \text { Neben- } \\ \text { kons. } & \text { vok. } & \begin{array}{c}\text { Neben- } \\ \text { kons. } \\ \text { vok. }\end{array} & \begin{array}{c}\text { Grenz- } \\ \text { vok. }\end{array} & \text { Suffix(e) }\end{array}$

$\begin{array}{lllllll}m & -u & -i^{\prime} t & -\hat{a} & -l & -\infty & -p \\ m & -u & -i^{\prime} t & -\hat{a} & -l & -i & -m\end{array}$

Bekanntlich sind in den meisten lappischen Dialekten mit quantitativen Wechseln oft auch andere prosodische Wechsel verbunden, vor allem im Verlauf der Intensität. Man kann sie jedoch - was auch allgemein getan wird - als Begleiterscheinungen ansehen. Das gilt auch für die vorliegende Arbeit. Über die Beziehungen zwischen Quantität und den übrigen prosodischen Erscheinungen im Lappischen s. näher Collinder, Neuphilologische Mitteilungen 1937 S. 97-118, MSFOu 74 S. 100, E. Itkonen $M S F O u$ S8 S. 1-52, Ravila Vir. 1961 S. 345-, Terho Itkonen FUF 35 S. 227-228. 
2.2.1. Der Stufenwechsel. Ausser für das Südlappische gilt der Stufenwechsel für alle anderen lappischen Dialekte. Im Umelappischen steht der einem kurzen Vokal einer betonten Silbe folgende Konsonantismus ausserhalb des Stufenwechsels. Im Übergangsgebiet zwischen Ume- und Pitelappisch, im Dialekt von Semisjaur, ist nur der zur $x x$-Reihe gehörende Konsonantismus nach einem kurzen Vokal einer betonten Silbe nicht dem Stufenwechsel unterworfen. In den nördlicheren Dialekten reicht der Stufenwechsel mit Ausnahme einiger Neutralisierungsfälle in alle Konsonantismen im Stamminnern an der Grenze zwischen 1. und 2., 3. und 4. Silbe usw. hinein. Wo er auftritt, ist überall mehr oder weniger quantitativer Wechsel des Vokalismus damit verbunden, der ausser von der Quantität des Konsonantismus auch abhängt von Quantität und Qualität des Vokalismus. Diachronisch gesehen dürfte der Quantitätswechsel des Konsonantismus ohne weiteres primär sein. Prinzipiell ist es zwar möglich und auch offenbar, dass ein gewisser Wechsel der Vokalquantität als phonetische Begleiterscheinung von da an auftrat, als der quantitative Stufenwechsel der Konsonanten sich voll ausgebildet hatte.

Der quantitative Stufenwechsel hatte zumindest in seiner frühesten urlappischen Form den Typus $\grave{x} x: x x, \dot{x}: x, \grave{x} y$ : $x y$, mit dem Ende der urlappischen Epoche aber oder bald danach fielen überall ausser im Südlappischen die schwache Stufe der $x x$-Reihe und die starke Stufe der $x$-Reihe zusammen, so dass sich die Wechselverhältnisse $\grave{x} x: \grave{x}, \grave{x}: x, \grave{x} y: x y$ ergaben (s. Bergsland Studia Septentrionalia II S. 15-, E. Itkonen $F U F 29$ S. 278-279, anders Ravila $F U F 33$ S. 318). Neben dem quantitativen Stufenwechsel begegnet im Lappischen ein qualitativer Stufenwechsel, der die Vertreter der ursprünglichen Einzelklusile und -affrikaten betrifft sowie dialektal auch in einigen Konsonantenverbindungen auftretende Klusile. Im Ostlappischen unterliegen auch die Vertreter der Einzelsibilanten dem qualitativen Stufenwechsel. Ferner zeigt das Ostlappische Spuren eines Stufenwechsels des Konsonantismus an der Grenze zwischen 2. und 3. Silbe. Der qualitative Stufenwechsel des Urlappischen vertrat den Typus $* \dot{k}:{ }^{*}{ }_{G}$ od. ${ }^{*} \gamma, *^{*} \dot{t}^{\prime}:{ }^{*} \dot{D} \dot{z}$ od. ${ }^{*} d \dot{z},{ }^{*} \dot{s}:{ }^{*} \mathrm{z}$ od. ${ }^{*} z$. Wir 
brauchen hier weder das umfangreiche Schrifttum zur Frage des lappischen Stufenwechsels zu referieren noch die Vertretung in den einzelnen Dialekten genau zu analysieren. S. K. B. Wiklund MSFOu 10 S. 71-122, MO 7 S. 197-239, 9 S. 171 -239, 13 S. 55-121, FUF 6 S. 5-16, Vir. 1921 S. 7--14; T. I. Itkonen $M S F O u$ 39; Frans Äinä JSFOu 23,25, FUF 6 S. 181-211, MSFOu 45, Vir. 1922 S. 1-29; Björn Collinder, Über den finnisch-lappischen Quantitätswechsel I (Uppsala 1929); Paavo Ravila MSFOu 62, Vir. 1951 S. 292-300, FUF 33 S. 285--325; Erkki Itkonen FUF 27 S. 137-167, MSFOu 88; Knut Bergsland Studia Septentrionalia II S. 1-53.

Mit ziemlicher Sicherheit kann der Stufenwechsel auf das Frühurfinnische zurückgeführt werden, doch ist vorläufig nicht entschieden, ob er eine noch ältere Erscheinung darstellt (s. Ravila FUF 33 S. 324--325; E. Itkonen, Suomalaisugrilaisen kielen- ja historiantutkimuksen alalta S. 60-61; Björn Collinder, Comparative Grammar of the Uralic Languages S. 215-218). Die meisten Forscher sind sich wohl heute darin einig, dass der Stufenwechsel im Frühurfinnischen nur die Klusile, die Affrikaten und eventuell die Einzelsibilanten betraf und dass erst in urlappischer Zeit auch andere Konsonanten in seinen Einflussbereich kamen.

Die Datierung des Stufenwechsels wird dadurch erschwert, dass bindende Kriterien fehlen, die zu einer Bestimmung des terminus post quem berechtigten. Es sind nur zwei Lautwandel bekannt, die älter zu sein scheinen als der Stufenwechsel im Lappischen und Ostseefinnischen und die prinzipiell als Kriterien herangezogen werden könnten, deren Glaubwürdigkeit aber aus später angeführten Gründen fragwürdig ist. Das eine ist der Wandel ${ }^{*} n m>{ }^{*} n n>n$, der in den Possessivsuffixen der 1. P. mit dem Koaffix $n$ eingetreten sein soll. Im Lappischen ist z.B. der Konsonantismus im Gen. Sg vor dem $n\left(<{ }^{*} n m\right)$ der 1. P. starkstufig, was auch vor $m$ der Fall ist, z.B. lpSk. Nom.Sg. + Px 1. Sg. $e^{J} t$ ť̌am $\left(<^{*}-m e \check{)}\right)$ : Gen.Sg. + Px 1. Sg. e $e^{J} t$ šan $\left(<^{*}-n \check{e}<{ }^{*}-n m e\right)$ 'Vater', vgl. Nom.Sg. + Px 2. Sg. kušsad $\left(<{ }^{*}-\right.$-ĕ $)$ : Gen.Sg. + Px 2. Sg. küžzaD (<*-nDĕ) 'Kuh' (Asbjörn Nesheim Studia Septentrionalia II S. 153-160). Dieselbe Situation herrscht auch in 
den Dialekten, wo sich $m$ anstelle von $n$ durchgesetzt hat, z.B. $\operatorname{lpN}$ Nom.Sg. + Px 1. Sg. ač čam : Gen. Sg. + Px 1. Sg. ač́čam, vgl. Nom.Sg. + Px 2. Sg. ač́čad: Gen.Sg. + Px 2. Sg. aččad 'Vater'. Auch im Ostseefinnischen steht vor dem Px

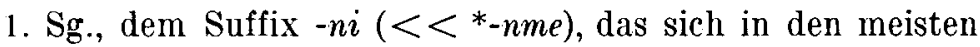
Dialekten in allen Stellungen durchgesetzt hat, normalerweise die starke Stufe, z.B. fi. poikani, tupani. Das genügt jedoch noch nicht als Beweis, denn es kann eingewandt werden, dass die starke Stufe hier von auf $-m i$ endenden Formen herrührt (poikami, tupami), wobei die heutigen Formen als eine Art Kontamination anzusehen wären (poikani $<{ }^{*}$ poirani + poikami). Die schwache Stufe im Dialekt von Iitti (Nom. Sg. + Px 1.Sg.tupam: Gen.Sg. + Px 1. Sg.tuvain) ist ebenfalls kein zuverlässiges Kriterium, demn hier kann die Analogie der übrigen Personen gewirkt haben, vgl. z.B. Nom.Sg. + Px 2. Sg. tupas: Gen.Sg. + Px 2. Sg. tuvans (Julius Mark MSFOu 54 S. 110-111, 119-120). Diese Formen tragen schwerlich zu einer eindentigen Entscheidung der Frage bei. Wichtiger ist dagegen, dass die starke Stufe in der alten finnischen Schriftsprache bei den Formen Gen., Akk.Sg., Nom. Pl. + Px 1. Sg. vorherrscht, während die entsprechenden Formen mit einem $\mathrm{Px} 2$. und 3 . Sg. sehr allgemein oder direkt in der Mehrheit die schwache Stufe haben (a.a.O. 218-219). Diese Beobachtung stimmt mit dem System im Lappischen gut überein. Es kann also für nöglich gehalten werden, dass die Stufenwechselverhältnisse im Frühurfinnischen folgendermassen aussahen:

$$
\text { Px 1. Sg. Px 2. Sg. Px 3. Sg. }
$$

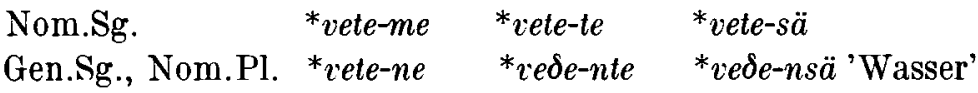

Der Stufenwechsel wäre somit eine jüngere Erscheinung

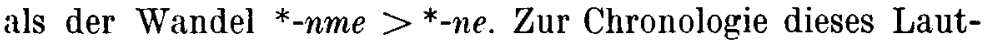
wandels lässt sich wiederum feststellen, dass das Mordwinische keinerlei Anhaltspunkt gibt, denn die durch den Typus Gen. Sg., Nom.Pl. + Px 1. Sg. tsoran 'Sohn' (contra Nom.Sg. + $\mathrm{Px}$ 1. Sg. t'soram) vorausgesetzte Entwicklung * ${ }^{*}$ me $>^{*}{ }^{*}$ nne 
$>n$ kann ebenso gut eine aus der Zeit der mordwinischen Eigenentwicklung stammende Parallelerscheinung zum finnisch-lappischen Lautwandel sein wie auf die Zeit ostseefinnisch-lappisch-mordwinischer oder finnisch-wolgaischer Gemeinschaft zurückgehen. Dagegen sind $n$ und $m$ im Px 1. P. im Syrj., Wog. und Selk. erhalten, zwar bezeichnet das ursprünglich für die Pluralität des Besitzes stehende $n$ im Syrj. nunmehr die Pluralität der Besitzer, z.B. syrj. Nom.Sg. + Px 1. Pl. vegunim, In., Ill.Sg. vęlanim (vgl. In., Ill. Sg. + Px 1. Sg. velam, vom Wort vel 'Pferd', ebenso In., Ill. Sg. + Px 2. Sg. velad : Px 2. Pl. velanid, Px 3. Sg.velas : Px 3. Pl.velanis); wog. Nom.Pl. + Px 1. Sg. sãlianum (vgl. Nom.Sg. + Px 1. Sg. sālim vom Wort sāli 'Rentier'); selk. Nom.Pl. + Px 1. Sg. loganim (vgl. Nom.Sg. + Px 1. Sg. logau vom Wort loga 'Fuchs') (S. Budenz, Ugrische Sprachstudien I, Pest 1869, $\mathrm{Az}$ ugor nyelvek összehasonlító alaktana, Budapest 18841894, S. 329-330; Paasonen MSFOu 22 S. 120-123; Wiklund MO 9 S. 1-11; Mark a.a.O. 245-251; Collinder, Indouralisches Sprachgut, Uppsala 1934, S. 26-29; Nesheim Studia Septentrionalia II S. 149-175; Liimola MSFOu 127 S. 237-241). Mit Vorbehalt kann hieraus die Schlussfolgerung gezogen werden, dass sich der Wandel $*_{-n m-}>-n$-frühestens nach der finnisch-permischen Zeit vollzogen hat. ${ }^{1}$ Da der Stufenwechsel eine jüngere Erscheinung zu sein scheint, könnte seine Entstehung aufgrund dieses Kriteriums in die finnisch-wolgaische oder die frühurfinnische Zeit verlegt werden.

Das zweite möglicherweise für die zeitliche Festlegung des Stufenwechsels geeignete Kriterium ist die Entwicklung des ostseefinnisch-lappischen suffixalen Labialvokals, indem der illabiale Vokal mit einem folgenden $v$ verschmilzt (E. Itkonen, Scandinavica et Fenno-Ugrica, Uppsala 1954, S. 183-191). In all den zu dieser Gruppe gehörenden Wörtern sind die Stufenwechselverhältnisse sowohl im Ostseefinnischen als auch

1 Es würde zu weit führen, wollten wir hier die Vokalverhältnisse von osfi.-wolg. *-nm- und der -nam- -Verbindungen der östlichen fiu. Sprachen klären; die Frage ist ausserdem irrelevant bei der Erörterung des Alters des Wandels ${ }^{*} n m>n$. 
im Lappischen konsequent auf demselben Stand wie in den ursprünglich auf Vokal auslautenden Wörtern, z.B. fi. pato : padon, $\operatorname{lp} \mathrm{N}$ buoddo : buodo; $\operatorname{lp} \mathrm{N}$ goč́čot : goččm (fi. kutsua: kutsun); lp N bossot: bosom 'blasen' (fi. puhua: puhun). Mit dieser Begründung könnte das Alter des Stufenwechsels noch präzisiert werden. Er scheint nämlich jünger zu sein als illab. Vok. $+v>$ lab. Vok. und somit also frühurfinnisch.

Keine der beiden genannten Lautveränderungen liefert ein völlig stichhaltiges Kriterium. Beidemal muss die Angleichung der Stufenwechselverhältnisse an im herrschenden System vorhandene Muster berücksichtigt werden. Für eine solche Angleichung liegen zahlreiche Beispiele vor in den ostseefinnischen Sprachen (vgl. fi. kukko 'Hahn', Part. Pl. tapoja 'Sitte', 3. Sg. Prät. antoi 'geben', in den südwestlichen Dialekten aber kuko, tavo, anno). Besonders klar auf der Hand lag die Möglichkeit der Angleichung so lange, wie der Stufenwechsel eine völlig mechanische phonetische Erscheinung war, und das war noch im Frühurfinnischen der Fall und auch im Urlappischen, vielleicht mit Ausnahme des letzten Stadiums. Phonologisch wurde der Stufenwechsel erst dann, als die in den nichtersten Silben eingetretene Entwicklung die Faktoren zunichte machte, die den Stufenwechsel als phonetische Erscheinung bedingt hatten, d.h. als der Stufenwechsel unabhängig wurde von der Offen- oder Geschlossenheit der folgenden Silbe.

2.2.2. Die Überdehnung. Zu einem vom Stufenwechsel unabbängigen quantitativen Wechsel führte im Lappischen die Überdehnung, worunter verstanden wird, dass nach betontem Vokal die starke Stufe der $x$-Reihe unter bestimmten Bedingungen quantitätsmässig wie die starke Stufe der $x x$-Reihe vertreten ist. Hierdurch kamen paradigmatische Wechsel zustande wie lp N Nom.Sg. suolo : Gen., Akk.Sg. sul'lu 'Insel', 1. Sg.Prs. mânâm : 1. Pl. mânnâp : Part.Prs. mân'ne 'gehen'. Überdehnung begegnet heutzutage im Pite-, Lule-, Norwegisch-, Inari- und Skoltlappischen. Wie schon Wiklund nachwies (MO 9 S. 193,13 S. 86-87, Vir. 1921 S. 8-), scheint die Überdehnung ursprünglich im Konsonantismus vor dem Kontraktionsvokal aufgetreten $\mathrm{zu}$ sein, doch sind für viele Dialekte Veränderungen anzunehmen, die die Überdehnung 
tilgten oder sie in Zusammenhänge brachten, in die sie ursprünglich nicht gehörte. Die Frage nach dem Alter der Überdehnung hängt eng zusammen mit der vom Alter der Kontraktion. Trotz der Unterschiede, die sowohl in der Überdehnung als auch in der Kontraktion zwischen den einzelnen Dialekten auftreten, kann doch zumindest eine teilweise historische Zusammengehörigkeit der Überdehnungserscheinungen in den verschiedenen Mundarten wohl kaum geleugnet werden. Die Kontraktionsvokale des Lappischen blicken sicher auf ein unterschiedliches Alter zurück. Die ältesten gehen - nach dem Umfang ihres Beleggebietes zu schliessen - auf das Urlappische zurück. Ob die Überdehnung bis ins Urlappische zurückgeführt werden kann, ist fraglich; sehr lange nach der urlappischen Zeit hat sie allerdings nicht aufkommen können, denn Voraussetzung für sie ist, dass die sie bewirkenden Kontraktionsvokale quantitätsmässig deutlich unterschieden sind von den nichtkontrahierten Vokalen. Näher dazu s. Wiklund a.a.O., Nielsen MSFOu 20 S. 17-33, Äimä Vir. 1922 S. 1329, Collinder MSFOu 74 S. 148-153, The Lappish Dialect of Jukkasjärvi (Uppsala 1949) S. 184-185, 191-192, Ravila MSFOu 62 S. 84-, E. Itkonen MSFOu 88 S. 113-116, 187 -192 .

2.2.3. Der Strukturtypenwechsel. Mit Strukturtypenwechsel ist in dieser Untersuchung der im Inarilappischen herrschende quantitative Wechsel zwischen dem Normaltypus, dem Dehnungstypus und dem Kurztypus gemeint sowie andere inarilappische quantitative Wechsel, die sich nicht auf der Basis des Stufenwechsels oder der Überdehnung erklären lassen. Nach Erkki Itkonen tritt der Normaltypus in ursprünglich zweisilbigen Wörtern auf, der Dehnungstypus in ursprünglich dreisilbigen Wörtern und in solchen fünfsilbigen, deren Stamm dreisilbig ist, und der Kurztypus in ursprünglich viersilbigen Wörtern und solchen mehrsilbigen, deren Stamm nicht dreisilbig ist (MSFOu 88 S. 54). Der Wechsel zwischen den Typen ist in gewissem Umfang phonologisch und kann auch eine morphologische Funk-

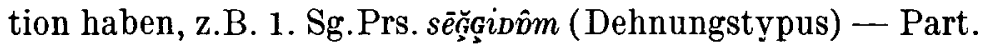
Perf. sẹ̀̆idôm (Kurztypus) 'schmaler machen' ( $<{ }^{*}$ sęqendam 


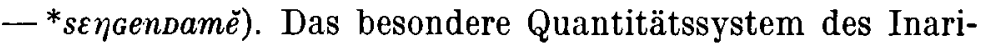
lappischen ist als Folgeerscheinung der verschiedenen Intensitätsverhältnisse entstanden, die in verschieden strukturierten Wörtern herrschten (a.a.O. 49-52).

2.2.4. Der Metaphoniewechsel. Ein beträchtlicher Teil der paradigmatischen Wechsel der Vokalqualität im Lappischen ist durch Metaphonie entstanden. Die ältesten Erscheinungsformen der Metaphonie sind bereits aus dem frühen Stadium des Urlappischen bekannt, wo die mittleren Vokale der ersten Silbe $e$ und $o$ durch Einwirkung des weiten Vokals der zweiten Silbe offener wurden, z.B. pesä $>{ }^{*}$ pes̀ä $>>\operatorname{lp~N}$ bresse 'Nest' (vgl. *vere $>{ }^{*}$ vęre $>>1 \mathrm{pN}$ vârrâ 'Blut'), kota $>{ }^{*} k$ ita $>>$ lpN goatte 'Zelt' (vgl. * polve $>$ * polve $>>\operatorname{lp~N}$ buol'vâ 'Knie'), und die offenen $\ddot{a}$ und $a$ wurden vor halbengen Vokalen der folgenden Silbe halbeng, z.B. ${ }^{*} k a ̈ t e ~>{ }^{*} k$ ete $>>\operatorname{lpN}$ giettâ 'Hand' (vgl. *äjmä $>*^{*} \ddot{a} m \ddot{a}>>1 \mathrm{pN} a i^{\prime} b m e$ 'Stopfnadel, Nadel'), *lakte $>{ }^{*}$ lok̀te $>>\operatorname{lpN}$ luok'ta 'Bucht' (durch Substitution auch kala $>{ }^{*}$ kola $>>\operatorname{lpN}$ guolle 'Fisch', vgl. jedoch ${ }^{*}$ vanțșa- $>{ }^{*}$ vaǹoza- $>>\operatorname{lp} \mathrm{N}$ vaz'zet 'gehen' $\sim$ tšerW $\beta a \cdot n$ -

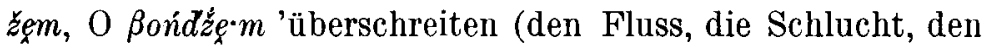
Graben, die Strasse, den Zaun usw.)') (E. Itkonen MSFOu 79 S. 56-75, Vir. 1950 S. 69-, Suomen Akatemia puhuu, Porvoo 1968, S. 54-55). Die älteste Metaphonie-Schicht hat jedoch keine paradigmatischen Wechsel bewirkt, sondern in den Fällen, wo man einen Wechsel erwarten würde, ist ein Ausgleich zugunsten der allgemeinen Vertretung erfolgt, z.B. $\operatorname{lpN} v \hat{a} r^{\prime} d e t$

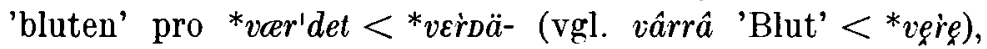
3. Sg.Prs. guos'ka pro ${ }^{*}$ goas'ka $<{ }^{*}{ }^{\prime} \wedge \hat{s} k a$ vom Verb guos'kât $\left(<{ }^{*}\right.$ kosskę-) 'berühren'. Zum letzten Beispiel ist jedoch zu sagen, dass das als Auslautvokal der 3. Sg.Prs. bei $\breve{a}$-stämmigen Verben auftretende $a$ vielleicht jünger ist als die Metaphonie (s. MSFOu 143 S. 190, Erkki Itkonen FUF 37 S. 110111).

Die spätere Metaphonie dagegen, die ebenfalls auf das Urlappische zurückgehen kann oder wenigstens auf eine späturlappische Prädisposition, hat paradigmatische Wechsel bewirkt. In den Fällen, wo die eine Voraussetzung der Metaphonie bildenden Vokaloppositionen in den nichtersten Silben 
geschwunden sind, ist der durch Metaphonie entstandene Vokalwechsel phonologisch geworden. Am häufigsten begegnet Wechsel durch Metaphonie heute im Süd- und Ostlappischen, am wenigsten im Lulelappischen, von wo aus der Anteil der Metaphonie sowohl in Richtung Süden als auch Osten stufenweise zunimmt.

Fälle von Metaphonie sind ausser in der betonten auch in der unbetonten Silbe bekannt. In allen Dialekten begegnet der urlappische Wechsel der gleichen Silbe $a \sim e$ und in den Westdialekten (Anzeichen davon möglicherweise auch im $\mathrm{Ko}$ lalappischen, s. S. 286-287) der Wechsel $o \sim u$, die teilweise durch die Metaphonie hervorgerufen sind, z.B. $1 p \mathrm{~N}$ Ill.Sg. guollai - Lok.Sg. guolest'Fisch' $<{ }^{*}$ kolajěn $-{ }^{*}$ koleste $<{ }^{*} k a-$ lajęn - *kalasta, Akt. goč čom - Part. Perf. goč'čum 'einladen'

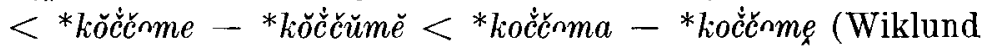
FUF 1 S. 81-99, 2 S. 41-71, E. Itkonen MSFOu 79 S. 113 $-114,151-156)$.

Über den Entstehungsmechanismus der lappischen Metaphonie ist nicht viel geschrieben worden. Zu regen Debatten kam es aber immer wieder im Zusammenhang mit den Erscheinungen der Metaphonie in den germanischen Sprachen und auch im Livischen, die stark an die lappische Metaphonie erinnern. Gegenstand der Auseinandersetzung war vor allem, ob die Metaphonie als Ergebnis von Kontaktassimilation so entstand, dass der Vokal der unbetonten Silbe auf den vorangehenden Konsonantismus "abfärbte", was wiederum auf den vorangehenden Vokal assimilierend wirkte, oder als Resultat einer Fernassimilation, indem die Artikulation des Vokals der zweiten Silbe ohne Vermittlung des Konsonantismus in der Artikulation des Vokals der ersten Silbe vorweggenommen wurde (zur Metaphonie im Livischen und in den germanischen Sprachen s. näher S. 350-361). Im Prinzip ist Metaphonie durch Kontaktassimilation dann möglich, wenn ihre Einwirkung in Palatalisierung oder Velarisierung zum Ausdruck kommt, in bestimmten Fällen auch in Labialisierung. Dass der Konsonantismus durch den Einfluss des vorderen oder hinteren Charakters des nachfolgenden Vokals palatalisiert oder velarisiert wird, ist aus zahlreichen Sprachen be- 
kannt, ebenso wie die Färbung des Vokals entsprechend einem folgenden palatalen oder velaren Konsonanten.

Wenn man das Lappische in den Bereich dieser im Grunde wichtigen und interessanten Frage bringen will, muss gleich festgestellt werden, dass Metaphonie im Lappischen nur teilweise Palatalisation und Velarisation ist. Ausser einer solchen die Artikulationsstelle betreffenden Metaphonie gibt es ferner eine des Artikulationsgrades, eine Grössenangleichung des bei der Vokalartikulation auftretenden Zungengaumenkanals an den Vokal der folgenden Silbe. Für die erste lappische Metaphonie lässt sich nichts anderes feststellen als ein Offenerwerden der mittleren Vokale $*_{e}$ und $*_{o}$ vor einem weiten Vokal der zweiten Silbe $\mathrm{zu}^{*} \varepsilon$ und $*_{n}$ sowie ein Engerwerden der Vokale $* \ddot{a}$ und $*_{a} a$ vor einem halbgeschlossenen Vokal der zweiten Silbe zu ${ }^{*} e$ und ${ }^{*} o$. Nicht einmal die Spur einer Palatalisierungs- oder Velarisierungsassimilation ist festzustellen, obwohl offenbar damals in der zweiten Silbe ${ }^{*} e$ und ${ }^{*} \ddot{a}$ noch palatal und ${ }^{*} o$ und ${ }^{*} a$ noch velar waren.

Auch die Metaphonie im Süd- und Ostlappischen betrifft grösstenteils den Artikulationsgrad. So entsprechen im südlappischen Dialekt von Röros den Vokalen $u o, i e, u$ und $i$ vor einem $*_{e}$ der zweiten Silbe $o \dot{a}, e a, a$ und $\ddot{a}$ vor einem $*_{n}$ der zweiten Silbe. Sowohl zwischen dem $*_{o} \underset{v}{\text { und }} *_{n}$ der zweiten Silbe als auch zwischen den Vokalreihen der ersten Silbe ist der Unterschied im Artikulationsgrad wesentlich. Der Konsonantismus ist in beiden Fällen konstant velar. S. Bergsland, Røros-lappisk grammatikk S. 82. Gustav Hasselbrink unterscheidet in den beiden durch Metaphonie entstandenen Hauptreihen des Mittelsüdlappischen, der palatalisierten und der velarisierten, eine offene und eine enge Unterreihe ${ }^{1}$ (Alternative Analyses of the Phonemic System in Central SouthLappish, Uralic and Altaic Series 49, The Hague 1965 S. 2529), z.B.

Pal. offen Inf. bel,dedh<* pè̀ne- 'erschrecken' eng 3. Sg.Prät. bil,dij $<{ }^{*}$ pělbǐ

1 Zur velarisierten Hauptreihe gehört ausserdem eine labialisierte Unterreihe, z.B. buldedh 'erschreckt werden' < *pĕì ŭ-. 
Vel. offen Part. Perf. beldeme < ${ }^{*}$ pëlDamĕ, berguo $<{ }^{*}$ pĕr̆gn 'Arbeit'

eng Part. Perf. bargeme < *pĕrGëmĕ 'arbeiten'

Die offene und enge Unterreihe entstanden als Resultat der Metaphonie, die den Artikulationsgrad betrifft.

In der ostlappischen Metaphonie unterscheidet sich deutlich die geschlossenere Metaphoniereihe von $*_{\breve{a}}\left(=*^{*} \breve{e}={ }^{*} e\right)$, $*_{u}$ und $* i$ der zweiten Silbe von der offeneren von $*_{a}, *_{n}$ und ${ }^{*} e$ (s. E. Itkonen MSFOu 79 S. 8-43), z.B. (Urlp. mit genauerer phonetischer Transkription bezeichnet)

2. Silbe

$*_{i} \quad$ 1. Sg.Prät.

$* u$ Part.Perf.Pass.

* $e_{2}$ 1. Sg.Prs.

$*_{\bar{e}}$ 3. Pl.Prs.

$*_{\bar{n}}$ 3. Sg.Imp.

$*_{\bar{a}} \quad$ 3. Sg.Prs.
$\operatorname{lpI}$

mōñnim

monnum

mōnp̣m

maǹ̀̀h

manòs

maǹà
lpSk. (Snk.) urlp.

\begin{tabular}{|c|c|}
\hline meñnem & $<*_{m e n ̀ n}(n) i m$ \\
\hline moǹnom & $<{ }^{*} \operatorname{men}(n) u m e$ \\
\hline$m \bar{\theta} n a m$ & $<{ }^{*}$ menem \\
\hline mäñne & $<{ }^{*} \operatorname{men}(n) \bar{e} k$ \\
\hline mānas & $<{ }^{*} m e \dot{n} \bar{n} s \vec{e}$ \\
\hline$m \bar{a} \breve{n} n n_{>}^{A}$ & $<{ }^{*}$ mén் $\bar{a}$ 'gehen' \\
\hline
\end{tabular}

Die Dimension offen - geschlossen als Eigenschaft von Vokalen dürfte wohl kaum etwas enthalten, was auf die Konsonanten abfärben könnte. Die Unterscheidung palatal velar ist aber umso schärfer, je geschlossener der Vokal ist, weshalb die engen Vordervokale ihre Umgebung leichter palatalisieren als die offenen, und die engen Hintervokale (labio)velarisieren leichter als es die offenen tun. Durch Palatalisierung und Velarisierung hat jedoch die auf den Artikulationsgrad wirkende Metaphonie nicht entstehen können. Im Südlappischen wie auch im Russischlappischen (schwächer auch im Inarilappischen) begegnet aber neben der eben genannten auch eine Metaphonie, die die Artikulationsstelle beeinflusst (s. z.B. oben). Doch steht im Ostlappischen vor engen Vokalen die enge Metaphonievariante und vor offenen Vokalen die offene, unabhängig davon, ob die durch Metaphonie entstandenen Vokale palatal oder velar sind. So liegen die Dinge in der Hauptsache auch im Südlappischen. Die einzige Möglichkeit ist, dass der Artikulationsgrad des Vokals 
der zweiten Silbe direkt auf den des Vokals der ersten Silbe eingewirkt hat, ohne Vermittlung des Konsonantismus.

Die Entstehung einer Metaphonie, die die Artikulationsstelle beeinflusste, durch Kontaktassimilation wirkt ebenfalls zumindest im Ostlappischen unwahrscheinlich. Soweit die zur Verfügung stehenden Quellen für das Inarilappische zeigen, wird der Konsonantismus dort nicht durch den Einfluss des Vokals der zweiten Silbe palatalisiert oder velarisiert, obgleich eine Metaphonie der Artikulationsstelle eindeutig im Vertreter von urlp. ${ }^{*} a$ in der ersten Silbe begegnet und andeutungsweise auch in den Vertretern von urlp. $*_{n}$ und $*_{o}$ (bei Äimä anch andeutungsweise in den Vertretern von urlp. ${ }^{*} \breve{a},{ }^{*} \breve{\imath},{ }^{*} \breve{o},{ }^{*} \breve{u},{ }^{*} \varepsilon$ und ${ }^{*} e$ ), z.B. 3. P.Sg. $t \dot{s} \bar{a} \bar{l} \dot{a}$ 'schreiben' (< urlp. *čala) -- pä̀ni 'Zahn' (< urlp. *paǹe), póaruì 'Bremse'

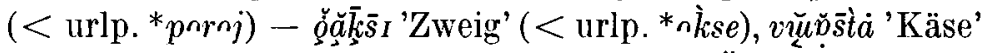
(<urlp. *os̀ta) - puarì 'gut' (< urlp. * pore); ( Äimä) Part.Perf. Pass. mònum 'gehen' (< urlp. *măǹumă) - 1. Sg.Prät. mòñim id. (< urlp. *mănim) usw. (E. Itkonen a.a.O.). Im Russischlappischen scheint die Metaphonie der Artikulationsstelle stärker zu sein als im Inarilappischen, doch auch im Konsonantismus zeigt sich eine Wirkung des nachfolgenden Vokals. Die Palatalisierung ist ausserordentlich stark bei den palatalen Konsonanten und am schwächsten bei den Labialen. Die Velarisierung kommt am deutlichsten beim $l$ zum Ausdruck, das vor einem hinteren Vokal als $n$ ausgesprochen wird, mitunter sogar wie $u$ (s. T. I. Jtkonen, Wörterbuch des Kolta- und Kolalappischen, Helsinki 1958, S. XXXI, XXXVI). Im Zusammenhang mit der Palatalisierung des Konsonantismus wurde auch das Ende des vorangehenden Vokals palatalisiert. Hierauf machte bereits Arvid Genetz aufmerksam, der die regressive Palatalisierung durch ein kleines untergestelltes $i$ bezeichnete, z.B. ta $i$ lle 'damals', soirme 'Tod' usw. (Wörterbuch der Kola-lappischen Dialekte nebst Sprachproben, Helsinki 1891, S. XII). Seit dem Jahre 1927 hat man diese Erscheinung bei einem Palatalklusil mit dem

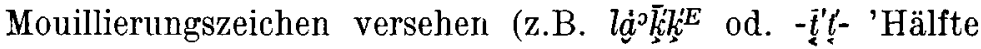
eines Gegenstandes'), während die anderen Konsonanten das Zeichen der Koronalisation erhielten (z.B. sịngn $n^{\dot{e}}$ 'Wort') mit 
Ausnahme der Labialen, bei denen sie überhaupt nicht bezeichnet wurde (T. I. Itkonen a.a.O. XXXVI). Wie Terho Itkonen feststellte, handelt es sich in erster Linie um einen auf die Grenze zwischen Vokal und Konsonant konzentrierten Mouillierungsübergang, der kaum jemals den ganzen Konsonanten betroffen hat, denn das koronalisierte $n$ hat sich ja getrennt vom ursprünglichen mouillierten $n$ erhalten (MSFOu 145 S. 84). Auch wenn die kombinatorische Koronalisierung resp. Palatalisierung des Konsonantismus mit der Metaphonie der Artikulationsstelle des Vokalismus zusammenhängen würde, kann also offenbar von einer direkten Kontaktassimilation nicht die Rede sein.

Im Südlappischen betrifft die Metaphonie der Artikulationsstelle systematisch sowohl den Vokalismus der vorangehenden Silbe als auch den dazwischen stehenden Konsonantismus mit Ausnahme von $\dot{n}, \breve{c}(\check{z}), j$ und $h$. Von allen anderen Konsonanten begegnet die vordere und die hintere Variante, die erstere vor einem ursprünglichen Palatalvokal und die letztere vor einem ursprünglichen Velarvokal. Regressive Palatalisierung und Velarisierung des Konsonanten treten auch im Anlaut des Wortes auf, je nachdem, ob der Vokal der ersten Silbe vor der Metaphonie palatal oder relar war, z.B. Rör. 1. Sg.Prät. b ötom 'kommen' $<{ }^{*}$ pnìim (das $i$ der 2. Silbe hat den Vokal der 1. Silbe palatalisiert, die Velarität von $b$ ist erhalten) $-b \ddot{o} k k<a j h$ 'dem Wind ausgesetzt sein od. werden' $<{ }^{*}$ pèे $\check{u}^{-}$( $b$ ist monilliert, weil der folgende Vokal ursprünglich palatal ist) (s. Bergsland, Roros-lappisk grammatikk S. 13). Nun liegt die Schlussfolgerung nahe, dass die teilweise Assimilierung der Konsonanten mit dem nächstfolgenden Vokal eine ältere Erscheinung ist als die Palatalisierung und Velarisierung der Vokale in der betonten Silbe; die Metaphonie der Artikulationsstelle wäre somit als eine durch den Konsonantismus herbeigeführte Kontaktassimilation zu betrachten. Das ist jedoch nicht die einzige Möglichkeit, es gibt noch eine andere Erklärung.

Gustav Hasselbrink teilt die Vokale der ersten Silbe in den mehrsilbigen Wörtern des Südlappischen in die folgenden vier Klassen: 
1. Palatal, durchweg vorn gebildet, z.B. $b$,iss,edh 'rösten' < *pisse-, lp $\mathrm{N}$ bâsset.

2. Velarisiert, nach hinten gleitend, z.B. $b$,issedh 'waschen' $<{ }^{*}$ pissă-, Ip N bâssât.

3. Velar, durchweg hinten gebildet, z.B. El.Sg. laddesde 'Rachitis' < *lăddă-.

4. Palatalisiert, nach vorn gleitend, z.B. El.Sg. ledd,esde 'Vogel' < *lădde-, lpN lod'de.

Palatale und velarisierte Vokale, deren Anfangsteil palatal ist, haben einen palatalisierenden Einfluss auf den vorangehenden Konsonanten, während velare und palatalisierte Vokale mit einem velaren Einsatz einen velarisierenden Einfluss auf den vorangehenden Konsonanten ausüben (Alternative Analyses S. 19). Hasselbrinks Darstellung ist synchronische Deskription, sie kann aber auch den Schlüssel für die historische Erklärung enthalten. Es ist nämlich möglich, dass die Metaphonie der Artikulationsstelle ursprünglich ausdrücklich den Schlussteil des Vokals beeinflusste. Die ursprünglich vorderen Vokale sind ja immer noch vollständig oder in ihrem Ansatz palatal (Gruppe 1 und 2) und die ursprünglich hinteren Vokale vollständig oder in ihrem Ansatz velar (Gruppe 3 und 4). Vielleicht vollzog sich die Metaphonie der Artikulationsstelle so, dass ein ursprünglich hinterer Vokal durch den Einfluss eines Vordervokals der zweiten Silbe einen palatalen Schlussteil erhielt und ein ursprünglich vorderer Vokal durch den Einfluss eines hinteren Vokals der zweiten Silbe einen velaren Schlussteil. Die Anfangsphasen der Entwicklung hätten also der Koronalisierungserscheinung des Russischlappischen sehr ähnlich sein können. Der Anfangsteil des Vokals, der seinen ursprünglichen Charakter beibehalten hatte, konnte später den anlautenden Konsonanten palatalisieren resp. velarisieren. Die Entwicklung hätte sich also folgendermassen vollzogen:

${ }^{*}$ priim $>{ }^{*} p o^{i}$ tim $>{ }^{*} p o^{i}$ tim $>{ }^{*}$ po ${ }^{i}$ tam $>b_{<}$tam, ${ }^{*}$ pè่Gü- $>{ }^{*}$ peGGu- $>{ }^{*} p e^{u} G G u->{ }^{*} \dot{p} e^{u} G G u->{ }^{*} \dot{p} o ̈ G G<a->$ bökti<a-. 
Für die zuletzt angeführte Erklärung sprechen zwei Umstände. Knut Bergsland teilt mit, die Palatalität des vorderen Stammkonsonantismus komme am stärksten in der Anfangskomponente der Dentalgeminata zum Ausdruck, z.B. mattajh wird màt $t \cdot i_{I}$ ausgesprochen ( $t$ ' stark mouilliert, $t$. schwach mouilliert) 'bitten, einladen, auf den Weg schicken' (Røros-lappisk grammatikk S. 43-44). Die vorderen Labialund Velarkonsonanten sind nicht palatalisiert. Der Verlauf der Mouillierung bei den Dentalgeminata lässt vermuten, dass sich die Palatalisierung ursprünglich auf die Grenze zwischen Vokal und Konsonant konzentrierte. Zweitens ist auch dann eine Metaphonie der Artikulationsstelle eingetreten, wenn zwischen den Vokalen $j$ oder ein absolut mouilliertes $\check{c}(\tilde{z})$ oder $\dot{n}$ steht, z.B. Rör. vuøjajh 'fahren' (<*vuoje-, lp N vuoggjet $) \sim$ vuojajh 'schwimmen' ( $<*^{*}$ vuojă-, lp N vuoggjât) (Bergsland a.a.O. 28), 3. Sg.Prs. čöččasta 'anhalten' ( $<{ }^{*}$ čuo ̌̀-

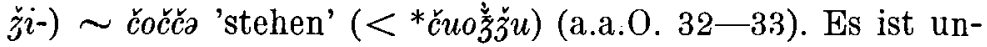
möglich, dass die Velarität erst vom Vokal der zweiten Silbe auf einen mouillierten Konsonanten oder $j$ übergeht und dann vom Konsonanten auf den vorangehenden Vokal und dass der Konsonantismus trotzdem seinen mouillierten oder palatalisierten Charakter behält. Die Palatalmetaphonie kann auch nicht durch diese Konsonanten weitergegeben werden, denn mouillierte Konsonanten und $j$ können ja nicht mehr mouilliert werden.

Möglicherweise handelt es sich also auch bei der die Artikulationsstelle betreffenden Metaphonie im Südlappischen ursprünglich um den Einfluss des Vokals der zweiten Silbe auf den der ersten oder eher die Fuge zwischen dem Vokal der ersten Silbe und dem folgenden Konsonanten, ohne dass der dazwischen befindliche Konsonantismus eine vermittelnde Rolle gespielt hätte. Natürlich haben sich im Konsonantismus gleichzeitig Änderungen vollziehen können, die einer Metaphonie entsprechen. In velarer Umgebung ist die Artikulation eines Konsonanten verständlicherweise velar und in palataler eben palatalisiert. Das ist jedoch etwas ganz anderes als das nach der Hypothese der Kontaktassimilation geschehende allmäh- 
liche Gleiten der Palatalität oder Velarität vom Vokal der zweiten Silbe an nach rückwärts, zunächst in den Konsonantismus und von dort in den Vokal der ersten Silbe.

Aufgrund der obigen Überlegungen kann es als wahrscheinlich gelten, dass die Metaphonie-Erscheinungen im Lappischen in ihrer Gesamtheit auf regressiver Fernassimilation beruhen.

2.2.5. Qualitativer Wechsel durch Kontralition. Ein als Grenzvokal auftretender Kontraktionsvokal weicht qualitätsmässig oft ab von einem in nichtkontrahierten Formen begegnenden erhaltenen Grenzvokal, was einen paradigmatischen Wechsel zur Folge hat, z.B. lp N Nom. act. mânnâm-1. Sg.Prät. mânnim 'gehen' (< *měnème - * *měnèjèm), suolo - Gen.Sg. sul'lu 'Insel' (<*solıj_* *solıjën). Die Kontraktion des Urlappischen betraf ursprünglich offenbar zumindest das $j$ an der Grenze zwischen 2. und 3. Silbe resp. 4. und 5. Silbe vor einer geschlossenen Silbe. Im Ostlappischen ist die auch in entsprechender Stellung befindliche Verbindung $n \tilde{n} \tilde{z}$ geschwunden und hat eine Kontraktion bewirkt. Ferner haben wir dialektweise Fälle, die auf eine Kontraktion durch den Schwund von ${ }^{*}{ }_{D},{ }^{*} z$ und ${ }^{*} v$ hinweisen, deren Geschichte aber noch nicht geklärt ist, z.B. $\operatorname{lpN}$ Attr. los'sis vom Wort lossâd 'schwer' (anders Collinder MSFOu 74 S. 160-161), 2. Du.Imp. diet'te 'wissen' (<*teteden, anders E. Itkonen Vir. 1955 S. 163164), Gen.Sg. sâr'va vom Wort sârves 'Rentierbock' (<*sĕrvazèn, vgl. jedoch Gen.Sg. oap'pas(â) vom Wort oappes 'Führer' < *np̀pazĕn), oar're 'Eichhörnchen' (vgl. lpSk. vŭăìrev). Analoge Kontraktion begegnet auch an der Grenze zwischen der 3. und 4. Silbe, z.B. 1. Sg.Prät. mui'tâlim $\left(<^{*} m u\right.$ ǔstělèjèm, s. MSFOu 143 S. 196), und besonders in den mittleren Dialekten des Lappischen auch vor der offenen Silbe, z.B. lpN Part.Prs. diet'te 'wissen' ( $<*$ teleje). Vgl. die in Abschnitt 2.2.2. S. 237 genannte Literatur sowie Wiklund $M O 7,9,13$; E. Itkonen MSFOu 79 S. 307-382, FUF 27 S. 137-167, Bergsland Studia Septentrionalia II S. 1-53.

2.2.6. Wechsel durch Verschmelzung von Vokalen. Der auf $i$ ausgehende Diphthong der unbetonten Silbe ist dialektweise monophthongisiert worden. In einigen alten Quellen lässt sich 
schwer schliessen, ob das Zeichen $\bar{\imath}$ wirklich einen langen Monophthong bezeichnet oder aber, ob es sich um den Diphthong $i j$ oder $i i$ handelt. Wiklund z.B. bezeichnet den Vertreter des Diphthongs $i j$ im Lulelappischen stets mit $\bar{\imath}, z$. .B. Gen.Pl. kà ti 'Hütte, Zelt', 3. Sg.Prät. kiesi 'ziehen' (s. z.B. Lärobok i lapska språket, 2. Aufl.; Grundström, Lulelappisches Wörterbuch, von Wiklund aufgezeichnete Paradigmen S. 1717-1918, in seinem ältesten Schaffen, z.B. der Laut- und Formenlehre der Lule-Lappischen Dialekte, Stockholm 1891, verwendet Wiklund das Zeichen 2 ), während die jüngeren Forscher folgende Bezeichnungen verwenden: $i i, i_{i}^{i}, i j, i^{j}$ usw., z.B. kiótiĭ, kóotij usw. (s. Grundström a.a.O.). Wirkliche Verschmelzungsvokale, die als Grenzvokale mit dem erhaltenen Auslantvokal des Stammes wechseln, begegnen im Skoltlap-

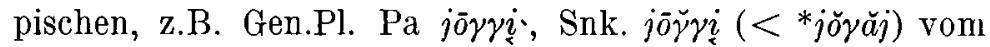
Substantiv $\mathrm{Pa} j \sigma^{\circ} \bar{k} k^{A}$, Snk. jos $\bar{k} k_{\varepsilon}^{A}$ 'Fluss', 3. Sg.Prät. Pa

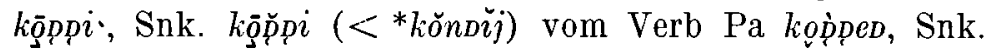
kopipe. 'töten' (E. Itkonen MSFOu 79 S. 383-385).

2.3. Die morphologischen Funktionen der Lautwechsel im Stamminnern

Alle oben behandelten Wechseltypen waren ursprünglich mechanisch und nicht distinktiv. In den heutigen lappischen Dialekten können sie sein:

1. nicht distinktiv, z.B. mit dem Stufenwechsel verknüpfter quantitativer Wechsel des Vokalismus der ersten Silbe in dem Wortpaar lp N Nom.Sg. kualli - Gen., Akk.Sg. ku.ali 'Fisch', 2. distinktiv

a. phonologisch, z.B. der Stufenwechsel in dem Wortpaar lp N Lok. Sg. guolest -- Ess.Sg. guollen $\left(<{ }^{*}\right.$ koleste *kolene),

b. morphologisch, z.B. der Stufenwechsel in dem Wortpaar $1 \mathrm{pN}$ Nom. Sg. guolle - Gen., Akk.Sg. guole $\left(<{ }^{*}\right.$ kole - *kolen, $\left.{ }^{*}-m\right)$,

c. lexikalisch, z.B. der Stufenwechsel in dem Wortpaar lp N adno 'grosser Fluss' - ano 'Onkel (mütterlicherseits)' $\left(<{ }^{*} \varepsilon \dot{n}^{n} \ldots{ }^{*} \varepsilon n n j\right)$. 
Ein morphologisch und lexikalisch distinktiver Wechsel ist immer gleichzeitig auch phonologisch distinktiv. Ein morphologisch distinktiver Wechsel kann entweder in der Flexion oder in der Derivation distinktiv sein. Der durch Derivation distinktive Wechsel ist gleichzeitig auch lexikalisch distinktiv, z.B. der durch Überdehnung hervorgerufene Wechsel in dem Wortpaar lpN 3. Sg.Prs. alla 'leben' - al'la

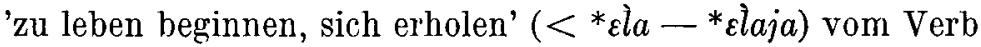
ellet und dessen Inchoativableitung al'lat. Ich beschränke mich im folgenden nur auf die Behandlung von morphologisch distinktiven Wechseln, wobei das Hauptaugenmerk auf die Flexionslehre gerichtet wird.

2.3.1. Wechsel in Flexionsfunktion. Die Distinktivität eines morphologische Funktion besitzenden Wortteiles, eines Suffixes oder eines zum Stamm gehörenden Elementes ist nicht immer absolut, besonders wenn die betreffende Sprache verschiedene morphologische Methoden nebeneinander anwendet. Eine Besonderheit, die die Formen A und B desselben Paradigmas voneinander trennt, kann zwischen den Formen A und $\mathrm{C}$ nichtdistinktiv sein. So unterscheidet sich z.B. die Form lpN 1. Sg.Prs. gulâm 'hören' von der 2. Sg.Prs. gulâk durch das Suffix, während der trennende Faktor zwischen den Formen gulâk und der 3. Pl.Prs. gullik kein Suffix ist, sondern der Stufenwechsel des Inlautkonsonantismus und der qualitative Wechsel des Grenzvokals. Um die morphologische Distinktivität der einzelnen Teile eines Wortes zu definieren und ein genaues Bild von der morphologischen Methode einer Sprache zu erhalten, müsste jede Form eines Paradigmas paarweise mit jeder anderen Form desselben Paradigmas verglichen und festgestellt werden, wodurch sich die Glieder eines jeden Paares voneinander unterscheiden. Das Ergebnis kann in einer Tabelle dargestellt werden, wo einer jeden Form im Paradigma eine Reihe und eine Spalte entspricht und wo im Schnittfeld der Reihe der Form A und der Spalte der Form B die trennenden Züge eingetragen werden. Das absolute Paradigma der im Stamm gleichsilbigen $e$-stämmigen Substantive im Lp N ergibt $\mathrm{z}$.B. folgende Tabelle (3) (Beispielwort jâkke 'Jahr'). 


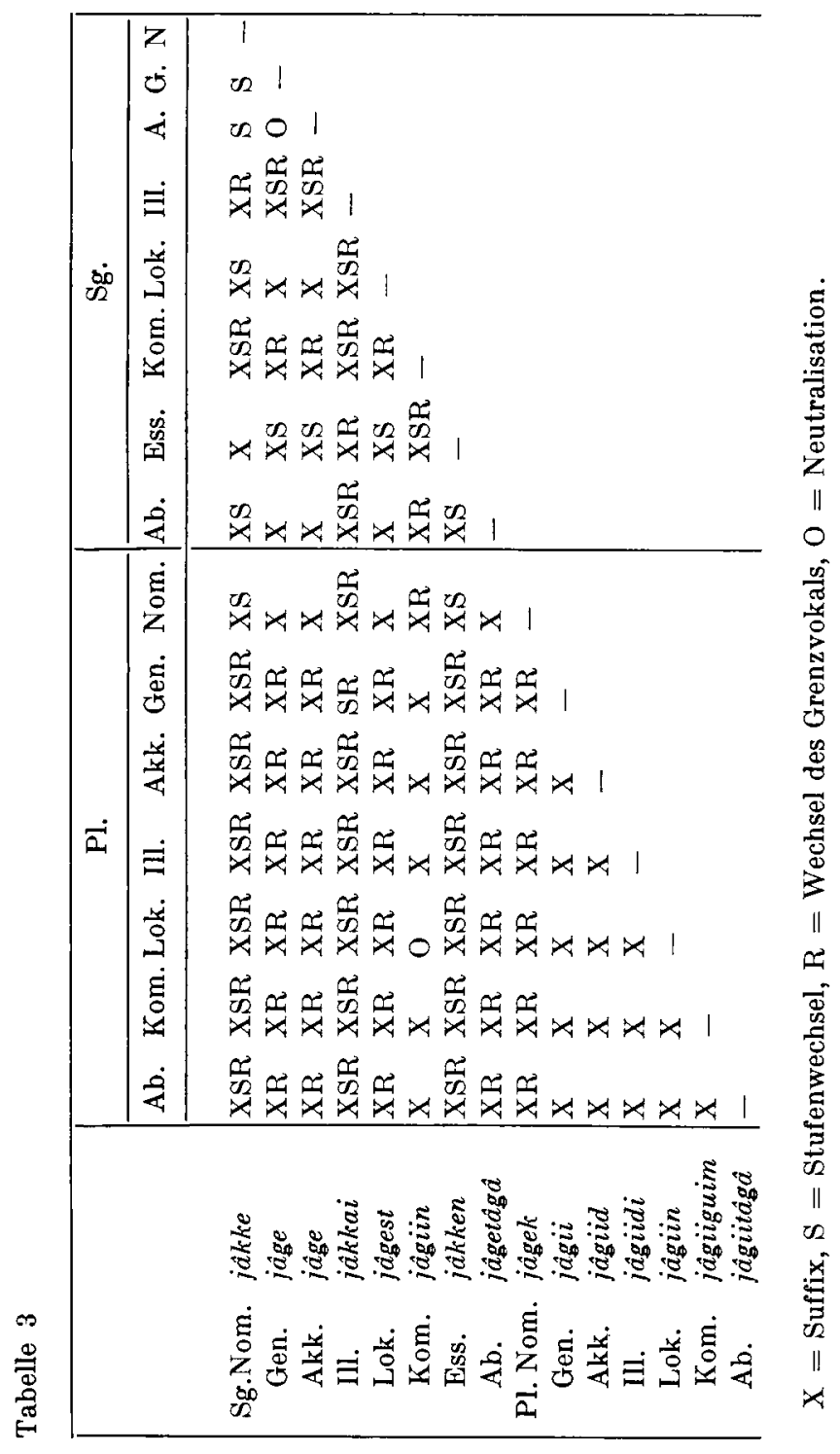


Die Tabelle zeigt, dass im absoluten Paradigma der $e^{-}$ stämmigen Substantive im Norwegischlappischen in 30 Paaren ausschliesslich das Suffix (X) den distinktiven Faktor ausmacht, neben dem Suffix wirkt in 8 Paaren der Stufenwechsel (XS) trennend, ein Suffix und der Wechsel des Grenzvokals (XR) in 37 Paaren sowie Suffix, Stufenwechsel und Wechsel des Grenzvokals (XSR) in 25 Paaren. Der blosse Stufenwechsel (S) ist nur in 2 Paaren disktinktiv, Stufenwechsel und Wechsel des Grenzvokals (SR) in 1 Paar. Neutralisiert (O) ist die Opposition in 2 Paaren.

Auch eine solche Überprüfung zeigt deutlich die interdialektalen Unterschiede. Das wird klar, wenn man die Resultate einer Untersuchung vergleicht, die an den Paradigmen der absoluten Deklination von $e$-stämmigen gleichsilbigen Substantiven im Süd-, Lule-, Norwegisch- und Skoltlappischen durchgeführt worden ist, s. Tabelle 4. Durch die Zahlen wird ausgedrückt, in wie vielen inneren Oppositionen des Paradigmas ein jedes Charakteristikum bzw. eine jede Gruppe von Charakteristika distinktiv ist und in wie vielen Fällen sich die Opposition neutralisiert hat. Da die Anzahl der Opposi-

Tabelle 4

\begin{tabular}{|c|c|c|c|c|c|c|c|c|}
\hline & \multicolumn{2}{|c|}{ Sd. (Rör.) } & \multicolumn{2}{|c|}{$\mathrm{L}(\mathrm{J})$} & \multicolumn{2}{|c|}{$\begin{array}{l}\mathrm{N} \text { (Ostfinn- } \\
\text { mark) }\end{array}$} & \multicolumn{2}{|c|}{ Sk. (Snk.) } \\
\hline & Stck. & $\%$ & Stck. & $\%$ & Stck. & $\%$ & Stck. & $\%$ \\
\hline $\mathrm{X}$ & 34 & 37 & 30 & 29 & 30 & 29 & 29 & 24 \\
\hline $\mathrm{XS}$ & - & - & 9 & 9 & 8 & 8 & 12 & 10 \\
\hline $\mathrm{XR}$ & 52 & 58 & 37 & 34 & 37 & 34 & 31 & 25 \\
\hline XSR & - & - & 25 & 24 & 25 & 24 & 17 & 14 \\
\hline XER & - & - & - & - & - & - & 3 & 3 \\
\hline XSER & - & - & - & - & - & - & 8 & 7 \\
\hline $\mathrm{S}$ & - & - & 2 & 2 & 2 & 2 & 3 & 3 \\
\hline $\mathrm{R}$ & 1 & 1 & - & - & - & - & 4 & 3 \\
\hline SR & - & - & 1 & 1 & 1 & 1 & 3 & 3 \\
\hline ER & - & - & - & - & - & - & 1 & 1 \\
\hline SER & - & - & - & - & -- & - & 4 & 3 \\
\hline O & 4 & 4 & 1 & 1 & 2 & 2 & 5 & 4 \\
\hline Insges. & 91 & 100 & 105 & 100 & 105 & 100 & 120 & 100 \\
\hline
\end{tabular}

$\mathrm{E}=$ Wechsel durch Überdehnung 
tionen infolge der verschieden grossen Zahl von Kasus in den einzelnen Dialekten schwankt, wurde auch der prozentuale Anteil eines jeden Charakteristikums, einer jeden Gruppe von ('harakteristika und der Neutralisation an der Gesamtmenge der Oppositionen errechnet.

Der Anteil der Suffixe als distinktiver Faktor nimmt nach Osten zu ab. Schon im Südlappischen verbindet sich mit der Verwendung von Suffixen meist ein Wechsel des Grenzvokals, ein Zug, der typisch ist für die fusionierende Methode. In den anderen Dialekten kommt der Stufenwechsel hinzı und im Skoltlappischen noch ein durch Überdehnung verursachter Wechsel (s. näher S. 273). Oppositionen, deren Suffixe nichtdistinktiv sind und die also die fusionierend-symbolische und die symbolische Methode vertreten, gibt es im Südlappischen nur eine. Der unterscheidende Faktor dabei ist der Wechsel des Grenzvokals (s. näher S. 254). Im Lule- und Norwegischlappischen beruht die Opposition in zwei Fällen auf dem Stufenwechsel und in einem Fall auf Stufenwechsel und Wechsel des Grenzvokals. Das Skoltlappische weist am meisten Oppositionen auf, die unabhängig sind von Suffixen und auf Wechsel im Stamminnern und deren Kombinationen basieren. Wie die morphologische Methode beim Übergang von den westlichen zu den östlichen Dialekten immer mehr Wechsel im Innern des Stammes vorzieht, geht anschaulich aus der Tabelle 5 hervor, wo die Ergrebnisse der Tabelle 4 dahingehend verwendet wurden, dass einmal jene oppositionen addiert wurden, in denen die Suffixe allein oder mit anderen Faktoren distinktiv sind (X, XS, XR, XSR, XER, XSER) und zum andern jene, wo die Suffixe nichtdistinktiy sind (S, R, SR, ER, SER).

\section{Tabelle 5}

\begin{tabular}{|l|r|r|r|r|r|r|r|r|r|}
\hline & \multicolumn{2}{|c|}{ Sd. } & \multicolumn{2}{|c|}{$\mathrm{L}$} & \multicolumn{2}{|c|}{ N } & \multicolumn{2}{|c|}{ Sk. } \\
\cline { 2 - 9 } & Stck. & $\%$ & Stck. & $\%$ & Stck. & $\%$ & Stck. & $\%$ \\
\cline { 2 - 9 } & & & & & & & & \\
Suffixe dist. & 86 & 95 & 101 & 96 & 100 & 95 & 100 & 83 \\
Suffixe nichtdist. & 1 & 1 & 3 & 3 & 3 & 3 & 15 & 13 \\
Neutr. & 4 & 4 & 1 & 1 & 2 & 2 & 5 & 4 \\
\hline Insges. & 91 & 100 & 105 & 100 & 105 & 100 & 120 & 100
\end{tabular}


In gleicher Weise könnten die Paradigmen aller Nominalund Verbalstammtypen dialektweise analysiert werden. Das erhaltene Resultat würde durch eine vollständige Analyse wahrscheinlich nur bestärkt. Interessanterweise gibt es in allen Dialekten, auch im Russischlappischen, letzten Endes sehr wenig Oppositionen, wo die Suffixe nichtdistinktiv sind. Dies beruht darauf, dass bei der Berechnung der Oppositionen an den Paradigmen auch die seltenen Formen ebenso berücksichtigt werden wie die üblichsten. Und doch kommt die symbolische Methode offenbar gerade bei den häufigsten Oppositionen zur Anwendung. Ich habe eine kleine Statistik ausgearbeitet über die Frequenz der Kasus im Norwegischund Skoltlappischen. Als Textmaterial wurden die Texte von Teil I von Qvigstads Lappiske eventyr og sagn S. $2-30$ verwendet, insgesamt 1816 Belege deklinierter Nomina, und die Seiten 163-184 der Sammlung von T. I. Itkonen, Koltanja kuolanlappalaisia satuja, die insgesamt 1080 Belege deklinierter Nomina enthalten. Das Ergebnis sieht folgendermassen aus:

Nom. Akk. Gen. Ill. Lok. Kom. Ess. Part. Abess. Instr. $\operatorname{lpN} \quad 44 \% \quad 22 \% \quad 15 \% \quad 8 \% \quad 6 \% \quad 3 \% \quad 2 \% \quad-\quad 0 \%-$ lpSk. $\quad 47 \% \quad 21 \% \quad 11 \% \quad 8 \% \quad 10 \% \quad 0,6 \% \quad 2 \% \quad 0,2 \% \quad 0,1 \% \quad 0,1 \%$

Obwohl die Statistik auf einem relativ einseitigen Material beruht, dürften die Zahlen nicht wesentlich von den wirklichen Frequenzverhältnissen abweichen. Die gemeinsame Frequenz von Nominativ, Akkusativ und Genitiv beträgt im Norwegischlappischen $81 \%$ und im Skoltlappischen $79 \%$. Gerade die Oppositionen zwischen diesen Kasus werden normalerweise mit symbolischen Mitteln ausgedrückt. Die gemeinsame Frequenz der genannten Fälle und des Illativs beträgt im Norwegischlappischen $89 \%$ und im Skoltlappischen $87 \%$. Von den 15 Oppositionen, wo im Skoltlappischen kein Suffix als distinktiver Faktor wirkt, bestehen 12 zwischen den Formen des Nom., Akk., Gen. und Ill. Sg. und Pl. Von den genannten Formen enthalten nur der Akk. und der Ill.Pl. ein Suffix. Obgleich es also in den Paradigmen relativ wenig 
Oppositionen ohne distinktive Suffixe gibt, kann der Anteil der symbolischen Methode im Text und in der Rede dennoch beachtlich gross sein, wie auch die Indexe der morphologischen Methode beweisen (Tabelle 2 S. 221).

Im folgenden kommen wir zur detaillierten Untersuchung jener Oppositionen, in denen ein Lautwechsel im Innern des Stammes flexionsmässig distinktiv ist. Untersucht werden das Südlappische, das Lulelappische, das Norwegischlappische, das Inarilappische und das Skoltlappische; die Wechsel sind in jedem Dialekt nach ihrem Ursprung gruppiert. Für die einzelnen Wechseltypen werden die auf S. 230 genannten $\mathrm{Ab}$ kürzungen verwendet. In Kursivschrift stehen die Abkürzungen für die Flexionsklassen. Die erste Buchstabenabkürzung steht für die Wortklasse $(N=$ Nomen, $V=$ Verb, $P P r .=$ Personalpronomen), die Ziffer bezeichnet die Silbenzahl des Stammes ( $2=$ gleichsilbig, $3=3-, 5-$ usw. ungleichsilbig) und der sich anschliessende Buchstabe den ursprünglichen Auslautvokal eines gleichsilbigen Stammes und den ursprünglichen Vokal der zweiten Silbe eines dreisilbigen Stammes, z.B. $V 2=$ 2 silbige Verben, $V 2 e=2$ silbige $e$-stämmige Verben, $N 3 \check{a}=$ 3 silbige Nomina, in deren 2. Silbe der Vertreter eines ă steht. Bei den Abkürzungen der Flexionstypen mit Kontraktionsstamm steht nach dem Kennzeichen der Wortklasse das Symbol des Ableitungselements, das charakteristisch ist für diese Typen, z.B. je, aje, ije, uje, s, ăk, dit. Eigens zu erwähnen ist $V l a-=l a-$ 'sein' und $V n=$ Verneinungsverb. Bei der Aufzählung mehrerer Flexionstypen sind weder die Abkürzungen für Wortklasse noch die für Silbenzahl des Stammes wiederholt angegeben, z.B. V2ă e 3 ije $n=$ gleichsilbige $\breve{a}$ - und $e$-stämmige, 3silbige und $i j e$-Verben sowie Verneinungsverb.

Südlappisch, Dialekt von Röros (Bergsland, Røros-lappisk grammatikk)

M 1

PPr. Akk.Sg. - Akk.Dıl: mânnam - munnam 1. P.;

El.Sg. - El.Du.: mânnasta - munnasta;

Kom.Sg. - Kom.Du.: mânnańa - munnaria.

V2e 2. Du.Prs. - 2. Du.Prät.: boåtaran - b<ötrŕran'kommen'; 
1. Pl.Prs. - 1. Pl.Prät.: boåtzuh - b<ötzuh;

2. Pl.Prs. - 2. Pl.Prät.: boâtára - b<ötára.

Vn 2. Pl.Imp. I - 2. Pl.Imp. II.: allara - uttaŕa.

Q

N2e Gen.Sg. - Ill.Sg.: goåtien - goåtan 'Zelt'.

PPr. Ill.Sg. - Gen. Dil.: munnan - munnan.

V2ă e 3 ije $n$ 2. Sg.Prs. - 3. Pl.Prs.: juzvk<astatth-juuk<aställieh ${ }^{1}$ 'saufen', boåtah - boåtieh, vardash — vardašech 'nachsehen, betrachten', čekkh - čekkieh 'stellen, setzen (einen Gegenstand, einmal)', ih-reeh N.

V2e 3 2. Sg.Prs. - 2. Sg.Prät.: boåtah - b<öth, vardashvardaših.

V2e 1. Sg.Prs. -- 1. Sg. Prät.: boåtam - b<ötəm;

1. Du.Prs. - 1. Du.Prät.: boåtien - b<ötan.

Vle- 2. Sg.Prs. , 3. Pl.Prs. -- 2. Sg.Prät.: leceh--lih.

Das Südlappische kennt weder Stufenwechsel noch vom Stufenwechsel abhängige oder unabhängige quantitative Wechsel. Phonologisch distinktive qualitative Wechsel gibt es zahlreich; darunter sind besonders die Metaphoniewechsel charakteristisch für das Südlappische. Die morphologische Distinktivität der qualitativen Wechsel ist jedoch relativ gering. Auch von den oben angeführten, auf Metaphonie beruhenden Oppositionen ist die bei den gleichsilbigen $e$ stämmigen Verben in der 2. Du., 1. und 2. Pl. auftretende Opposition Prs. - Prät. sclten. Normalerweise wird das Präteritum der gleichsilbigen Verben mit einem Suffix ausgedrückt (2. Du.Prät. b<ötajijtaran, boåtiejijtaran usw., s. Bergsland a.a.O. 161-164). Der Übergang der morphologischen Funktion rom Suffix auf den Stamm beruht bei den Fällen von Metaphonie auf der Ausgleichung der Vokaloppositionen in den nichtersten Silben. In den Beispielen vertritt das a der

1 Hier wie auch in mehreren folgenden Formpaaren kommt die Opposition auch als Metaphoniewechsel des Inlautvokalismus zum Ausdruck, da aber der Wechsel des Grenzvokals als primăr und der Metaphoniewechsel als eine Folgeerscheinung dessen anzusehen ist, wird die Opposition nur im Zusammenhang mit dem Wechsel durch Kontraktion angeführt. Auch in der Folge wird ein Metaphoniewechsel nicht berücksichtigt, falls die Vokalopposition der folgenden Silbe erhalten ist. 
zweiten Silbe ein *e (Prs.-Formen boataran usw., 2. Pl.Imp. I allara), ein $*_{i}$ (Prät.-Formen $\left.b<o ̈ t a r a n ~ u s w.\right), ~ e i n ~ * \breve{a ~(P P r . ~}$ Akk.Sg. mannam usw.) und ein ${ }^{*} u$ (2. Pl.Imp.II uttaŕa, PPr. Akk. Du. munnam usw.).

Von den Wechseln durch Kontraktion begegnet Gen.Sg. $i e-$ Ill.Sg. a (goåtien - goåtan) nur in $e$-stämmigen Substantiven, deren Ill.Sg. denselben Kontraktionstypus hat wie im Ostlappischen (Rör. goåtan, I koat'p̣n, Sk. Snk. kŏăătta < urlp. *kntajën). Der Ill.Sg. der anderen Substantive vertritt einen anderen, nichtkontrahierten Typ, z.B. Ill.Sg. becerg<asa vom Wort bearga 'Fleisch' (s. Bergsland a.a.O. 93, 96, 112 und CSIFU I S. 77-85). Die durch Kontraktion bewirkten Oppositionen Prs. - Prät. (boåtam - b<ötzm usw.) sind ebenso selten wie die entsprechenden durch Metaphonie bewirkten (Oppositionen (s. oben). Die bei den Personalpronomina vorhandene Opposition Ill.Sg.--Gen.Du. (munnan - munnən) geht zurück auf die verschiedenen Kontraktionsvokale in der zweiten Silbe. Die erstere Form hat als unregelmässig zu gelten. Nach Bergsland stammt das $a$ aus dem Illativ der $e$-stämmigen Substantive (Roros-lappisk grammatikk S. 96). Das a im Typ munnan vertritt ein Kontraktions- *u $\left(<{ }^{*}\right.$ mŏǹu $<$ ${ }^{*}$ moǹnjĕn) (vgl. Ravila MSFOu 62 S. 88-89). Eine regelmässige, auf dem Wechsel zwischen nichtkontrahiertem und kontrahiertem Vokal beruhende Opposition liegt vor in 2. Sg.Prs.3. Pl.Prs. (boåtah - boåtieh usw.) (s. MSFOu 143 S. 331335). Neutralisiert ist die Opposition im Präsens der o-stämmigen Verben und des Verbs la-, da der nichtkontrahierte und der kontrahierte Vokal identisch sind (2. Sa., 3. Pl.Prs. daịtah 'machen', lech). Die in der Flexion des Verneinungsverbs begegnende Opposition 2. Sg.Prs. - 2. Pl. Prs. iheceh beruht auf unregelmässigem Wechsel zwischen nichtkontrahiertem und kontrahierten Vokal. Über das im Westlappischen in den singularischen Personen auftretende $i$ (im, ih, ij) s. Bergsland Vir. 1960 S. 327.

Das Obige trifft zu für den Dialekt von Röros, der zur Dialektgruppe von Jämtland gehört. Ein wenig anders liegen die Dinge in der Gruppe von Assele. Gustav Hasselbrink stellte in seiner Studie "Metaphonie als distinktives und re- 
dundantes Merkmal im Südlappischen»(CSIFU I S. 168177) fest, dass die Metaphonie in den mittleren südlappischen Dialekten nur bei der Trennung von Nomen actionis und Part.Perf. bei $e$-stämmigen Verben "binnenparadigmatisch" distinktiv ist, z.B. Nomen act. vuo jneme - Part.Perf. vuejneme 'sehen' 1 sowie bei undeutiger Aussprache in einigen anderen Fällen. Eine "zwischenparadigmatische», lexikalische Distinktivität gäbe es dagegen häufiger. Nach Lagercrantz begegnet in der Mundart von Vefsen, die zur Dialektgruppe von Åsele gehört, der Unterschied zwischen Nomen act. und Part.Perf. bei $\boldsymbol{e}$-stämmigen Verben auch in der Vokalqualität

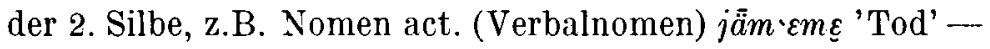
Part. Perf. jăm ‘àm $\xi^{\prime}$ gestorben' (Sprachlehre des Südlappischen, Kristiania 1923, S. 80).

Lulelappisch, Dialekt von Mittel-Jokkmokk (Grundström, Lulelappisches Wörterbuch S. 1717-1918)

$\mathrm{S}$

N2 Nom.Sg. - Gen.Sg., Nom.Pl.*: māanna $\underset{\sim}{a}-m \bar{a} n \bar{a}$ ' Kind',

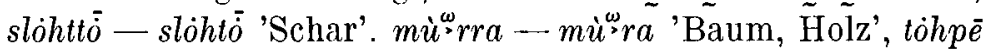

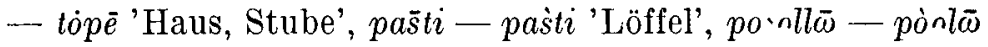
'Knopf', jötō ${ }^{h}$ tahka - jöto ${ }^{h} t a k \bar{a}$ 'der übliche Weg des Wanderzuges' 2;

Ill.Sg. - Gen.Pl. (alle ausser $2 e$ und 2o): mãannài - mānài,

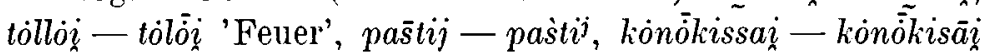
'König';

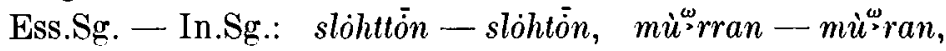

1 Im Jämtlandlappischen ist wenigstens nach Bergsland die Metaphonie in diesem Fall nicht distinktiv, sondern die Opposition kommt auch in den Suffixen zum Ausdruck, Part.Perf. - $m_{<} a$, Nomen act. -ma (Røroslappisk grammatikk S. 161, 232).

${ }^{2}$ Wegen des konsonantischen Auslauts des Yom.Sg. ist der Stufenwechsel zwischen dem Nom.Sg. und dem Gen.Sg. (Nom.Pl.) im vier-

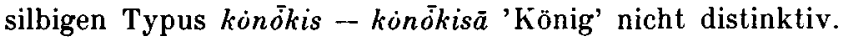

${ }^{*}$ Im Auslaut der mit ${ }^{*}$ bezeichneten Formen kann ein ${ }^{h}$ zu hören sein, wobei dann der Wechsel im Stamminnern für die diesbezüglichen Formpaare redundant ist. 
kònhtēn - kǒn̆tēn 'Lappenzelt', pas̄tin - pas̀tin, pörromussan - pórromusān od. -muhan - -muhān 1 '(etw. zum) Essen'; El.Sg. - Nom.Sg. + Px 3. Sg.: mānàs - mãnnnàs;

Nom.Sg + 2., 3. Px - Gen., Akk.Sg. + 2., 3. Px: ${ }^{o} n b b^{\circ}$ àt -_onppāt, onbb'ās —onppās 'Schwester'.

Naje Nom.Sg. - Ill.Sg., Gen.P1.: ólmāi - òlàmài 'Mann';

In.Sg. - Ess.Sg.: ólàmān-òlmān.

$\operatorname{PPr}$ Nom.Pl. - Gen.Pl.: midja - miinā 'wir'.

V2ă $e$ 2.-3. Sg. Prs.: kulä* -kullā 'hören', pònt $\bar{a}^{*}-$ pònhtā 'kommen'.

V2ă 3. Sg.Prs. -- 2. Sg.Imp. I: kullā-kulā

V2e n 2. Sg.Imp. I - 2. Du.: pòntēe-pònhté, ale $\bar{e}^{*}-$ allē.

$V 2 o$ 2. Sg.Prs., Imp. I -- 2. Du.Imp. I: kòrr $\bar{\omega}^{*}-k^{\circ} r r \bar{\omega}$ 'nähen'.

$\mathrm{E}$

V2 2. Du.Imp. I -- Part.Prs.: kullēe kullēe 'hören', pònhtē

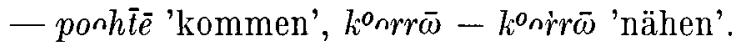

M 2

V2e o 3 Part.Perf. — Nomen act.: pònhtām-pònhtēm, körrum - kònrrōm, mālēstam-mälēstim 'kochen'.

$\mathrm{C}$

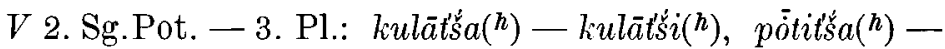

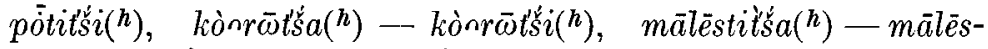

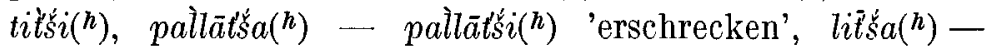
$\operatorname{lits} i(h)$.

V2ă e 3. Sg.Prs. - 3. Pl.Prs., 2. Sg.Prät. - 2. Du.Imp. I: kullā - kulli* - kullēe, pònhtā-- pōhti* - pònhtē.

V2o 3. Sg., 3. Pl.Prs.*, 2. Sg. Prät.* - 2. Du.Imp. I: körru $-k^{\circ}$ nrrī.

${ }^{1}$ Das $h$ unterlag nicht dem quantitativen Wechsel, der Stufenwechsel aber geht aus der Vokalquantität der 4. Silbe hervor: in einer die starke Stufe voraussetzenden Form ist sie kurz, in einer die schwache Stufe verlangenden Form lang. Ein solcher Vokalwechsel ist regelgemäss nach einem etymologisch kurzen Vokal der 3. (resp. 1.) Silbe und bei etymologisch kurzem $a$ und $o$ nach einem an der Grenze zwischen 3. und 4. (resp. 1. und 2.) Silbe stehenden, zur $x$-Reihe gehörenden Konsonantismus. 
I3 2. Sg.Prs., 2. Sg.Imp. - 3. Pl.Prs., 2. Sg. Prät.: mālēsta $\left.{ }^{h}\right)-\operatorname{mā}{ }^{2} s t i\left(^{h}\right)$.

Vle- 2.*, 3. Sg.Prs. - 3. Pl.*: là, lä - li (3. Pl. auch lä $(h))$. I'n 2. Sg.Prs. - 3. Pl.: $i\left({ }^{h}\right)-\bar{a}\left({ }^{h}\right), \grave{a}, \dot{\varepsilon}$.

$\mathrm{S}+\mathrm{E}$

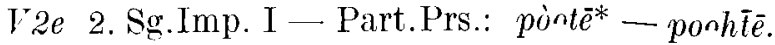

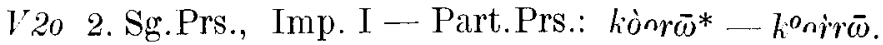

$\mathrm{S}+\mathrm{C}$

Ns Nom.Sg. -- El.Sg.: pàl'kès - pàlakãs 'Fusssteig';

In.Sg. - Ess.Sg.: pàlakān - pàl'kèn.

V2 1. Sg.Prs. - 1. Sg.Prät.: lókōw-lónkive, pòntàupóhtiw, kìnrīw - körruw;

2. Sg.Prs., 2. Sg. Imp. I - 3. Pl.Prs., 2. Sg.Prät.: kulā(h)

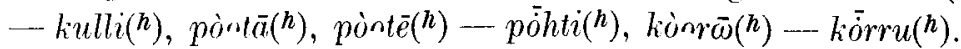
$\mathrm{S}+\mathrm{E}+\mathrm{C}$

Nuje Nom.Sğ. - Ill., Sg., Gen.Pl.: pòntscì - ponhtsui 'Rentier';

In.Sg. - Ess.Sị.: ponhtsun--- pòtsín.

V2ă 2. Sg.Prs., 2. Sgr.Imp. I - Part.Prs.: kulä* - kul̀ ē.

V2e 2. Sg.Prs. - Part.Prs.: pòntä $\bar{a}^{*}$ ponht̄̄e.

$\mathrm{S}+\mathrm{M} 2$

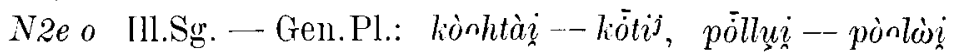
$\mathrm{E}+\mathrm{C}$

Y2 3. Pl.Prs.*, 2. Sg.Prät.* - Part.Prs.: kulli-kullēe,

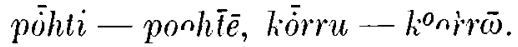

A

PPr. Nom.Sg., Du. - Gen.: món-mū Sg., mò̀ ${ }^{J}$, mōdjamunnu Du.

V2 2. P1.Imp. I. 一 Inf.: kullit - kullat, kòtit - kòtēt' Netze stricken', körrut - konrr⿳亠丷厂t.

Von den morphologisch distinktiven Wechseln im Innerı des Stammes ist der Stufenwechsel im Lulelappischen am häıfigsten. Die morphologische Distinktivität des Stufenwechsels ist eine Folge dessen, dass am Ende des Wortes ein oder mehrere Laute geschwunden sind und das Suffix entweder ganz verschwand oder einem anderen Morphem ähnlich

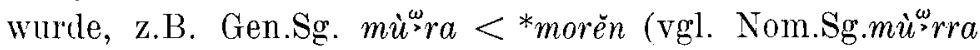




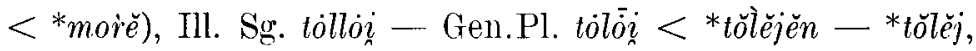
Ess.Sg. kiònhtēn - In.Sg. kŏntēn <*kntene - *knpesne, El.Sg. mānàs - Nom.Sg. + Px. 3. Sg. mānnàs < $<$ manaste *mañase, Nom.Sg. + Px 2. Sg. ${ }^{\circ}$ obb àt - Gen.Sg. + Px 2. Sg.

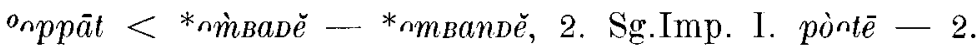
Du.Imp. I. pòshtē $<{ }^{*}$ pndek $-{ }^{*}$ pnieven (kontr.).

Durch die Überdehnung wird das Part.Prs. von der 2. Du. Imp. I der im Stamm gleichsilbigen Verben getrennt. In der ersteren Form steht in der 2. (oder 4.) Silbe ein Kontraktionsvokal, der die Überdehnung bewirkt hat. Auch der Grenzvokal der letzteren Form kann das Ergebnis einer Kontraktion sein, hat aber keine Überdehnung veranlasst (s. MSFOu 143 S. 271-273 und die dort genannte Literatur).

Im Lulelappischen begegnet kein morphologisch distinktiver Metaphoniewechsel in der betonten Silbe. Metaphonie in der unbetonten Silbe ist nur in einer Beleggruppe distinktiv geworden (Part.Perf. - Nomen act.: pùnhtäm - pònhtēm < ${ }^{*}$ prìtamĕ $-{ }^{*}$ priteme $<{ }^{*}$ potame $-{ }^{*}$ potama $)$. Metaphonische Erscheinungen an sich sind dem Lulelappischen nicht völlig fremd, doch begegnen sie fast ausschliesslich auf phonetischer Ebene (s. Collinder MSFOu 74 S. 57-71).

Die durch Kontraktion bewirkten qualitativen Wechsel des Grenzvokals sind in der Konjugation morphologisch distinktiv. In einigen Fällen wechselt der nichtkontrahierte Vokal mit dem kontrahierten, z.B. 3. Sg.Prs. kulla - 3. Pl.Prs., 2. Sg.Prät. kulli<*kŭla - *külèjet, *külèjèt, in anderen Fällen handelt es sich um den Wechsel zwischen zwei verschiedenen Kontraktionsvokalen, z.B. 2. Sg.Prät. kòorru -

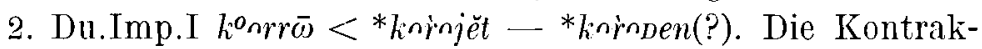
tion in der 3. Pl.Prs. und der 2. Sg.Prät. der im Stamm ungleichsilbigen Verben ist sekundär (s. MSFOu 143 S. 196, 336).

Von den übrigen Wechseln im Innern des Stammes sind die in der Flexion der Personalpronomina auftretenden unregelmässig ( Nom.Sg. mòn - Gen. mū; mòñ ${ }^{J}-m u n n u<{ }^{*} m o ̈ n n j-$ ${ }^{*}$ mŏǹnjèn). Vorläufig ungeklärt sind die in der zweiten Silbe der 2. Pl.Imp.I auftretenden $i$ und $u$, wodurch diese Form getrennt wird von dem auf dem regelmässigen $\check{a}^{-}, e$ - und $o$-Stamm beruhenden Infinitiv (vgl. a.a.0. 181-182). 
Norwegischlappisch (Nielsen, Lærebok i lappisk I, Grammatikk)

$\mathrm{S}$

N2 Nom.Sg. - Gen., Akk.Sg. 1: oab'ba - oabba 'Schwester', jokkâ - jogâ 'Fluss', guolle — guole 'Fisch', bâs'ti - bâsti 'Löffel', fabmo - fāmo 'Kraft', bel'ku -- bëlku 'Schlagklotz';

Nom.Sg. + 2., 3. Px - Gen., Akk.Sg. + 2., 3. Px: oab'bad, $-d e,-d e k,-s,-s k \hat{a},-s e k-o a b b a d,-d e,-d e k$ usw.

N2 a $i$ u Ill.Sg. - Gen.Pl.: bal'kai - balkai 'Lohn', bâs'tii

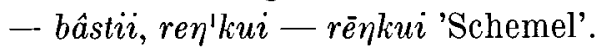

N3ă $i$ Nom.Sg. - Gen., Akk.Sg.: gāmâs - gābmâs 'Beinhaut des Rentiers', geris - gerris 'Lappenschlitten'.

Naje Nom.Sg. -- Ill.Sg., Gen.Pl.: âlmai - âl'bmai 'Mann'.

V2ă e 1.Sg.Prs. — Part.Perf.: gulâm-gullâm 'hören', dieđam - diettam 'wissen'.

V2ă o 1. Sg.Prs. - Nomen act.: gulâm - gullâm, goččom goč́čom 'rufen, befehlen'.

$V 2 o$ 3. Sg.Prs. - 2. Sg.Imp.: divvo - divo, -ó 'reparieren'. $\mathrm{E}$

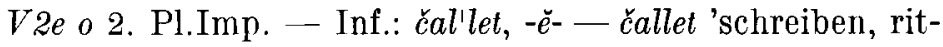
zen', goar'rot, -̌̌- - goarrot 'nähen'.

V2o 3. Sg.Prs. - 1., 2. Du.Imp., Part.Prs.: divvo - div'vo;

1. Pl.Prs. - 1. Pl.Imp.: divvop-- div'vop, - o- $^{\prime}$

M 2

N2o (ohne Stufenwechsel) Ill.Sg. - Gen.Pl.: ak'sui ak'šoi 'Axt'.

V2e o Part.Perf. - Nomen act.: giel'dam - giel'dem 'verbieten', sup'pum (auch 1. Sg.Imp.) - suop'pom 'werfen (mehrmals)'.

$\mathrm{C}$

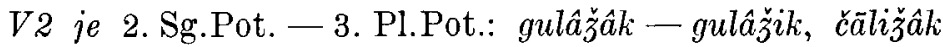

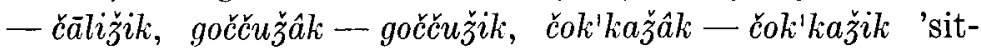

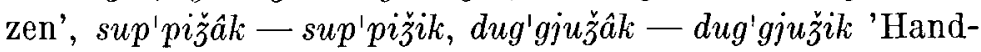
arbeiten machen'.

V2 3. Sg.Prs. - 1. Du.Prs., 3. Pl. Prät.: doalla - dōlli 'halten', čalla - čālli, goč čo - goččcu;

1 Betrifft nicht die vier-, sechs- usw. silbigen $\check{a}$-Stämme. 
1. Sg.Prät. - Nomen act.: gullim - gullâm, dīttim - diettem, divvum-- divvom.

V2ă e 1. Sg.Prät. - Part.Perf.: gullim - gullâm, dĩttim diettam;

1. Du.Imp. - 2. Du.Imp., Part.Prs.: gul'lo-gul'le, âd'no - $\hat{d} d^{\prime} n e$ 'gebrauchen'.

V3 1. Sg.Prs., Part.Perf., Nomen act. - 1. Sg.Prät.: mui'tâlâm - mui'tâlim 'erzählen';

2. Sg.Prs. - 3. Pl.Prs., 2. Sg.Prät.: mui'tâlâk-mui'tâlik;

V3 la- 1. Du.Prs.- 1. Du.Imp.: mui'tâled $n i-m u i^{\prime} t \hat{a}-$ lød'no, led'ni-led'no.

Vn 1. Sg. - 1. Du.: im- $\vec{e} m$;

1. Du.Imp. - 2. Du.: âl'lo-âl'le.

$\mathrm{S}+\mathrm{E}$

V2e $n$ 2. Sg.Imp. - 2. Du. Imp., Part.Prs.: ânĕ, -e $\hat{a} d^{\prime} n e, \hat{a l e \check{~}}-\hat{a} l^{\prime} l e$.

V2o 2. Sg.Imp. - 1., 2. Du. Imp., Part.Prs.: goarŏ, -ogoar'ro.

$\mathrm{S}+\mathrm{M} 2$

N2e o Ill.Sg. — Gen.Pl.: jâkkai - „âgii 'Jahr', dâkkuidâgoi 'Tat, Werk'.

N3e o Nom.Sg. - Gen., Akk.Sg.: nâger - nâkkar 'Schlaf, Traum', àlos - allus 'saftige, untere Schicht der Baumrinde'.

V2e 1. Sg.Prs. - Nomen act.: čālam-čallem.

V2o 1. Sg.Prs. - Part.Perf.: divom-divvum.

$\mathrm{S}+\mathrm{C}$

V2 1. Sg.Prs. - 1. Sg.Prät.: gulâm-gullim, čălamčăllim, suoppom-sup'pum;

2. Sg.Prs. - 3. Pl.Prs., 2. Sg.Prät.: doalâk - dōllik 'halten', gieldak - gil'dik, goarok - gōrruk;

1. Du.Prs., 3. Pl.Prät. - 2. Sg.Imp.: gaw'dni-gāwnâ 'finden', cab'mi - cabmé, -e 'schlagen', divvu - divo, -o.

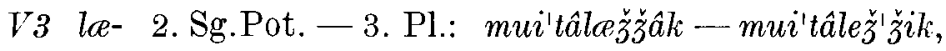

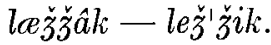

$\mathrm{S}+\mathrm{E}+\mathrm{C}$

Nuje Nom.Sg. - Gen., Akk. Sg.: suolo-sul'lu 'Insel'. 
V2ă 2. Sg.Imp. - 1., 2. Du.Imp., Part.Prs.: gulâ - gul'lo, gul'le.

V2e 2. Sg.Imp. --. 1. Du.Imp.: $\hat{a} n \breve{e},-e-\hat{a} d^{\prime} n o$.

$\mathrm{E}+\mathrm{C}$

V2 1. Du.Prs., 3. Pl.Prät. - 1., 2. Du.Imp., Part. Prs.: dōlli--doal'lo, doal'le, dïli---diet'to, diet'te, gōrru--goar'ro.

V2ă e 3. Sg.Prs. - 1., 2. Du. Imp., Part.Prs.: doalla-doal'lo, doal'le, čalla - čal'lo, čalle.

V3 3. Pl.Prät. - Part.Prs.: mui'tâleggïi--mui'tâlag'gje.

$A$

PPr. Nom.Sg., Du. - Gen.,Akk.: mōn--mū, dōn-- dū, sōn-sī; moai - mon'nu, doai-.. dod'nu, soai-- sod'nu.

$\mathrm{E}+\mathrm{A}$

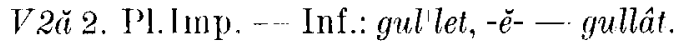

Das ()bige ist nach der in gewissem sinne normativen Grammatik von Nielsen dargestellt. In Wirklichkeit vertreten gerade diesen Stand vor allem die norwegischlappischen Dialekte von Ostfinnmark und speziell die Mundart von Polmak (Nielsen, I ærebok i lappisk I S. VII).

Die morphologischen Funktionen der Wechsel im Stammesinnem entsprechen im Norwegischlappischen in grossen Zügen denen im Lulelappischen. Stufenwechsel und ein auf Kontraktion bermhender qualitutiver Wechsel herrschen vor. Auch die Üherdehnung - allein oder in Verbindung mit anderen Faktoren - hat eine beachtliche Menge morphologisch distinktiver Wechsel verursacht. Die morphologische Distinktivität der Metaphonie dagegen kommt nur in einigen Oppositionsfällen $e(i)-a$ und $o-u$ der zweiten Silbe vor.

Der grösste Teil der Differenzen zwischen dem Lule- und dem Norwegischlappischen erklärt sich aus der unterschiedlichen Vertretung von auslautend $m, n$ und $k$ in diesen Dialekten. Abweichend vom Lulelappischen ist im Norwegischlappischen der Stufenwechsel zwischen dem Nom. und Akk. Sg. der im Stamm zweisilbigen Substantive distinktiv, was auf dem im $\mathrm{LpN}$ in vielen Formgruppen, u.a. im Akk.Sg., begegnenden Schwund des anslautenden $m$ beruht (jokkâ jogâ <*jŏkě — jŏgĕm), sowie zwischen dem Nom.Sg. und 
dem Gen.-Akk.Sg. von im Stamm dreisilbigen, dem Stufenwechsel unterliegenden Substantiven mit einem $\breve{a}$ oder $i$ in der zweiten Silbe (gämâs - gäbmâs < *kaměs — *laà̀èsèn, $-m$ ). Dass im Lulelappischen ein urspr. auslantendes $m>w$, ein sekundär auslautendes $m$ aber $\equiv$, hat die Distinktivität der Suffixe in den Formpaaren 1. Sg.Prs. -- Part.Perf.

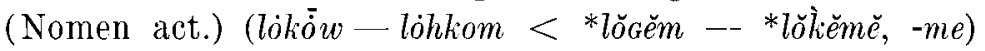
und 1. Sg.Prät. -- Part.Perf. (Nomen act.) (1. Sg. Prät. lóhkiw $<$ *lŏkĕjĕm) erhalten, während das - ungeachtet seines Ursprungs - erhaltene $-m$ im Norwegischlappischen dem Stufenwechsel und dem qualitativen Wechsel des Grenzvokals in den entsprechenden Fällen eine morphologische Funktion verliehen hat (logâm-lokkâm, lokkim-lokkâm). Da weiterhin der Schwund des auslautenden ${ }^{h}(<k)$ im Lulelappischen in vielen Fällen eine morphologische Distinktivität des Wechsels im Innern des Stammes bewirkt hat, wurde im Norwegischlappischen durch das nicht geschwundene $-k$ die Opposition besser in den Suffixen bewahrt, z.B. 3. Sg.Prs. lpL kullā, N gulla - 3. Pl.Prs., 2. Sg.Prät. L kulli, N gullik. Andererseits wiederum ist der Schwund des Auslaut-n im Norwegischlappischen regelmässig, im Lulelappischen nur auf einige Formgruppen beschränkt, worauf dann Oppositionen beruhen wie die im Norwegischlappischen herrschenden 3. Sg.Prs. čalla - 1. Du.Prs. čălli und 1. Du.Imp. čal'lo-2. Du.Imp. (Part.Prs.) čal'le und die entsprechenden lulelappischen Oppositionen vom Typus pònhtä - póhtin und porrōon -- pórrē.

Durch unregelmässige Überdehnung wurden die Oppositionen verursacht, die zwischen der 2. Pl.Imp. und derr Inf. sowie der 1. Pl. Prs. und der 1. Pl. Imp. herrschen (čal'letčallet, divvop - div'vop) (s. MSFOu $143 \mathrm{~S} .182$ ).

In den mittleren und westlichen Dialekten des Norwegischlappischen sieht die morphologische Distinktivität der Lautwechsel im Stamminnern in gewissem Masse anders aus als in den Ostdialekten. Die Unterschiede beruhen hauptsächlich darauf, dass auslautend $p, t$ und $k>k$, und zwar in Karasjok, Outakoski und heute immer allgemeiner auch anderwärts im Kirchspiel von Utsjoki sowie östlich von Polmak wenigstens 
in Maattivuono und Kaakkuri (Ravila JSFOu 45,6 S. 1011) und $>t$ in den Dialekten von Kautokeino, Loppa, Repperfjord, Kvänangen, Lyngen, Balsfjord und Karesuando (a.a.O.), sowie $m$ und $n>n$ anderwärts ausser in Polmak und eventuell noch in einigen kleineren Küstendialektinseln (a.a.0. 17). Ferner würden $-B,-G$ und $-D>-k$ in Karasjok und Outakoski, aber zu $-t$ in den meisten norwegischlappischen Westdialekten, wie in den Mundarten von Kautokeino, Repperfjord, Kvänangen, Lyngen und Balsfjord (a.a.O. 14-15). Wegen dieser Lautwandel ist eine grosse Anzahl von Oppositionen zwischen Suffixen geschwunden, während gleichzeitig Lautwechsel im Innern des Stammes morphologisch distinktiv wurden; falls für das Stammorphem in beiden Formen dasselbe Allomorph verwendet wurde, sind sich die Formen in ihrer Phonemgestalt gleich geworden, z.B. Kautokeino ${ }^{1}$ (als distinktiver Faktor S) Nom.Pl. oabbat - Nom.Sg. + Px 2. Sg. oab'bat, 2. Sg.Prs. gulât - 1. Pl.Prs., Inf. gullât; (C) 3. Pl.Prs., 2. Sg.Prät., 2. Pl.Imp. gullit - 1. Pl.Prs., Inf. gullât; (nicht dist.) Ess.Sg., Nom.Sg. + Px 1.Sg. oab'ban < oab'ban - oab'bam, Nom.Pl.Adj., Nom.Sg.Komp. nuorât < nuorâk - nuorâb 'jung'.

Inarilappisch (E. Itkonen $M S F O u$ 79, 88, Msk) ${ }^{2}$ $\mathrm{S}(+\mathrm{T})$

N2 Nom.Sg. - Gen.,Akk.: mềे $\hat{x}-m \hat{\varepsilon} r \hat{p}$ 'Meer', ńù̀è -

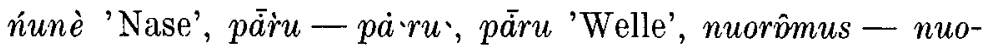
rômùz 'der jüngste';

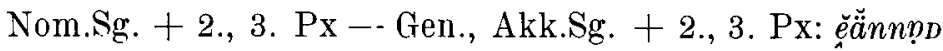
- eä̆nnp 'Mutter'.

N3ă $i$ o Nom.Sg. - Gen., Akk.: tuo'nôr - tŭŏndôr 'Fjell', nanòs - nannōs 'fest, stark', hĕ

Naje Nom.Sg.--Ill.: almàì' -- alimăì 'Mann';

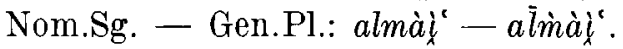

${ }^{1}$ Die Schreibweise folgt der Orthographie von Nielsen, mit Ausnahme der Auslautkonsonanten.

${ }^{2}$ Herr Prof. Erkki Itkonen hat mir freundlicherweise seine inarilappischen Aufzeichnungen zur Verfügung gestellt, wofür ich ihm hiermit meinen besten Dank ausspreche. 
V2 1. Sg.Prs. - Part.Perf.: tōp' $\underline{p} m$-- topp'p̣m 'schliessen', t'śàlàm - tśăllumm 'schreiben', polt'òm — poîtt'om 'drehen, durchwühlen';

3. Sg.Imp. -- 3. Du., Pl.: tap'òs - tapp'os, tšàlus - tšáăllus, polt'os - poltt'os;

3. Pl.Prs. - 2. Sg.Pot.: äseh - äzzēh 'wohnen', tšäleh -

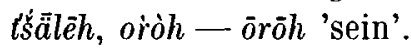

V2ă o 1. Sg.Prs. - Nomen act.: tōp'p̣m-topp'p̣m, polt'òm - poltt'om.

$V 2 e$ (in der 1. Silbe ein etym. kurzer Vok.), V2o 1. Sg.Pot.

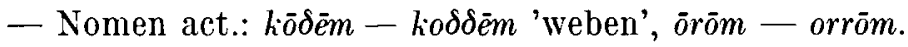

$V 20$ (in der 1. Silbe ein etym. kurzer Vok.) 2.Sg.Prs. 3. Pl.: polt'ò - politt'oh;

3. Sg.Prs. - 2. Sg.Imp.: poltt'o-polt $\grave{o}$.

$\mathrm{E}$

V20 (in der 1. Silbe ein etym. kurzer Vok.) 3. Sg.Prs. 2. Du.Imp., Part. Prs.: orò --- orrō.

$\mathrm{T}$

N2a ă o 3 Ill.Sg. - Ess., Gen.-Akk.Sg. + 1. Px: mêrîn -

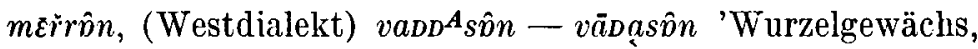
Wurzel'.

$V 20$ (in der 1. Silbe ein etym. kurzer Vok.) 1. Sg.Prs. 1. Sg. Pot.: polt' òm - poltt'ōm, oròm - ōrōm;

2. Sg.Prs. - 2. Sg.Pot.: polt'òh - poltt'ōh, oròh - örōh.

V3 je la- 2. Sg.Pot. - 3. Pl.Pot.: almòtt'ióñzéh - almòtt i-

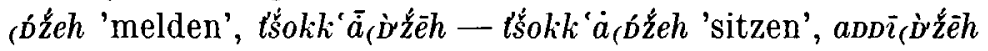

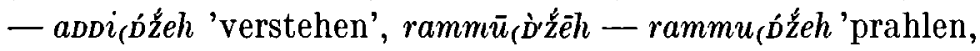

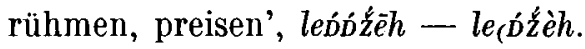

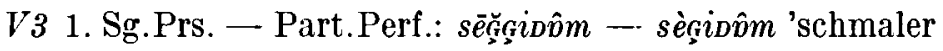
machen'.

Vje 1. Sg.Prs. - Part.Perf., Nomen act.: t'sokk' $\vec{a} m-$ t'škk'àm, tuhhim - tuhhim 'taugen', rammüm-rammum.

M 1

V2e o (in der 1. Silbe ein etym. langer Vok.) 1. Sg.Prät. Nomen act. (2o auch Part.Perf.): tšã än̆num.

$\mathrm{M} 2$

N2e Part.Sg. - Nom.Sg. + 2. Px: ennis - ęănnppd. 
V2e 3 Part. Perf. - Nomen act.: tśāillam - tšáĭlim, almòtt'pqm-almòtt'im.

$\mathrm{C}$

V2ă e 3 je 1. Du.Prs. -- 1. Dı.Imp.: ässēn- $\operatorname{assōn,~piūi-~}$

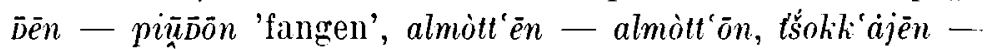

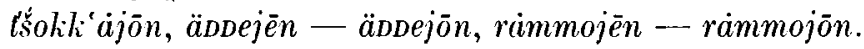

V2ă, V2o (in der 1. Silbe ein etym. kurzer Vok.) 1. Sg. Prät. - Nomen act., Part. Perf.: topp'im - top'pôm, puitt'um poitt'om.

$V 2 e 3$ 1. Sg.Prät. - Part.Perf.: tśállim -- tšăllỵm, almì̀tt'im - almòtt'p̣m.

V2e (in der 1. Silbe ein etym. kurzer Vok.) 1. Sg. Prät. Nomen act.: piū̄oim-- piū̄ōem.

$V 23$ je 3. Pl.Prät. - 2. Du.Imp. (V2 auch Part.Prs.):

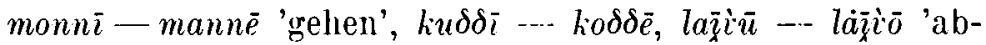

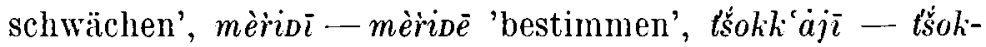

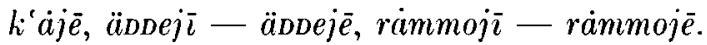

V3 2. Sg.Prs. - 3. Pl.Prs.: alìnōt ąh-almōt eh;

Vje 3. Pl.Prs. - 2. Sg.Prät.: tśskliajeh-tśokk'ajih, ändejeh -- äDDejih, rämmojeh - rammojih.

Vije uje 3. Sg.Prs. - 2. Sg.Imp.: tohhe- -- tuhh $\bar{\imath}$, rämmōrammī.

Vla- 3. Sg.Prs.-3. Pl.: lī-lī.

$\mathrm{S}+\mathrm{E}$

$V 2 e$ o (in der 1 . Silbe ein etym. kurzer Vok.) 2. Sg. Imp. -

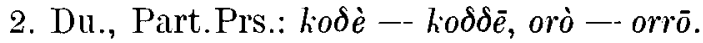

$\mathrm{S}+\mathrm{M} 2$

N3e Nom.Sg. - Gen., Akk.: naŭvèr-nahhär 'Schlaf, 'Traum'.

V2e 1. Sg.Prs. - Nomen act.: tóálàm-t tśăllim.

$\mathrm{S}(+\mathrm{T})+\mathrm{C}$

$V 2$ 1. Sg.Prs. - 1. Sg.Prät.: mōṇ̣m - mōñnim, hodìm lī̄ंठim, polt'òm-- puiltt'um;

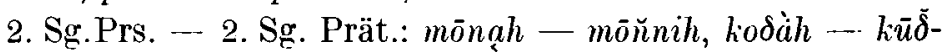
$\delta i h$, polt'òh-·pultt'uh;

1. Sg. Prät. - 1. Sg. Pot.: topp'im - tapp'èm, tśăllim -

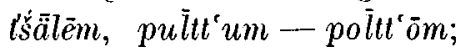

2. Sg.Prät. - 2. Sg.Pot.: topp'ih - tapp'ēh, tśăllih -t'śälēh, puìltt uh - politt'ōh. 
$V 2 \check{a} e$ 1. Sg.Pot. - Part.Perf., (V2ă, V2e in der 1. Silbe ein etym. langer Vok.) Nomen act.: äzzēm - (Part.Perf.,

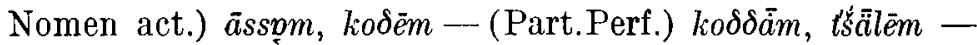
(Part.Perf.) tšállụm, (Nomen act.) t'śăllim.

$V 2 a$ e, V2o (in der 1. Silbe ein etym. langer Vok.) 2. Sg. Prs.

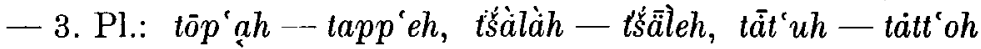
'wollen'.

V2ă 3. Sg.Prs. - Pot. neg.: kulà - kūli 'hören'.

\section{$\mathrm{S}+\mathrm{E}+\mathrm{M} 1$}

$V 2 e o$ (in der 1. Silbe ein etym. langer Vok.) 3. Pl. Prät. -

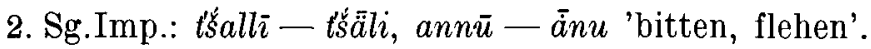
$\mathrm{S}+\mathrm{E}+\mathrm{C}$

Nuje s Nom.Sg. - Ill.: pojarul’ - porruị' 'Bremse'. välis - vällps 'Wal'.

Nuịe Nom.Sg. - Gen.Pl.: sụ̌̆

Năk Nom.Sg. - Gen., Akk.Sg.: mie'st

V2ă, V2e o (in der 1. Silbe ein etym. langer Vok.) 2. Sg.Imp.

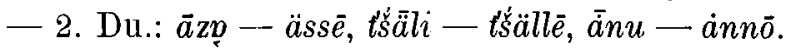

$\mathrm{E}+\mathrm{M} 1$

$V 20$ (in der 1. Silbe ein etym. langer Vok.) 3.Sg. Prs.

3. Pl.Prät.: á̀̀̀u-annū.

$\mathrm{E}+\mathrm{C}$

V2ăe, V2o (in der 1. Silbe ein etym. langer Vok.) 3. Sg.Prs. -

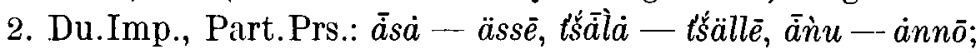
$V 2 a ̆ e, V 2 o$ (in der 1. Silbe ein etym. kurzer Vok.) 3. Sg.Prs.

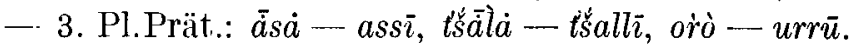
$\mathrm{T}+\mathrm{C}$

N2e 3 Ill.Sg. - Ess.: niuñän - nunnēn 'Nase', (Ost- und Norddial.) nahhäran - nohàrin.

$V 2 a \check{e}, V 2 o$ (in der 1. Silbe ein etym. langer Vok.) 1. Sg.Prs.

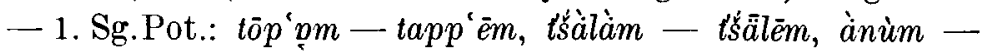
änōm;

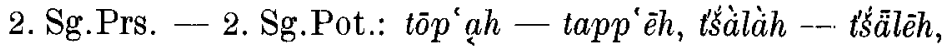
$\grave{a} n \grave{u} h-\bar{a} n o ̄ h$.

$V 2 a \check{a} e, V 2 o$ (in der 1. Silbe ein etym. kurzer Vok.) 2. Sg.Imp.

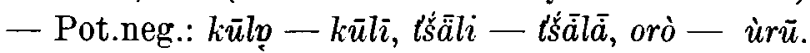

V2 3 3. Pl.Prs. - 2. Sg.Prät.: $\ddot{a} s e h--\bar{a} \breve{s} s i h, k o \delta \grave{e ̀ h}--k \bar{u} \check{\delta}-$

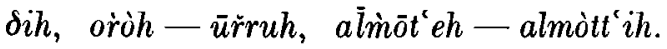

V3 2. Sg.Prs. - 2. Sg.Prät.: aìmōt'ąh-almòtt'ih. 
A

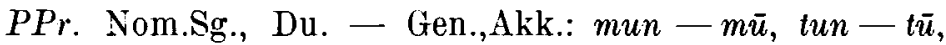

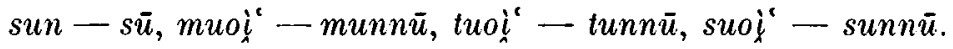

Im Inarilappischen begegnen bedeutend mehr Formpaare mit morphologisch distinktivem Lautwechsel im Innern des Stammes als in den westlappischen Dialekten. Der in gewissem Ausmass heterogene Strukturtypenwechsel hat im Inarilappischen eine sehr vorherrschende Stellung inne. Beeinflusst wurde die Entwicklung der Strukturtypen durch ursprüngliche Qualität und Quantität der Laute, die Stufenwechselverhältnisse, die ursprüngliche Silbenzahl des Wortes usw. Weil in einem und demselben Paradigma nur ein kleiner Teil aller im Inarilappischen vorhandenen Strukturtypen auftreten kann, ist auch die Anzahl der morphologisch distinktiven Strukturtypenwechsel beschränkt.

In allen Fällen, wo der Strukturtypenwechsel morphologisch distinktiv wurde, basierte die Opposition ursprünglich ausschliesslich auf den Suffixen. Die Opposition zwischen Ill. und Ess.Sg. beruht auf der im Ill.Sg. eingetretenen abweichenden Quantitätsentwicklung, die von E. Itkonen näher behandelt worden ist (s. MSFOu 79 S. $373-374,88$ S. 119-

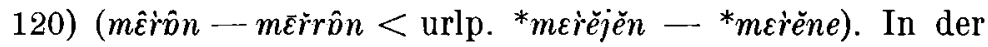
1. und 2. Sg.Prs. und Pot. der o-stämmigen Verben ist es die unterschiedliche Quantitätsstruktur, die die Formen voneinander trennt. Beidemal vertritt der Konsonantismus die ursprünglich schwache Stufe, doch der zur $x x$ - und $x y$-Reihe gehörende Konsonantismus ist in der Form des Pot. gedehnt. Die Prs.-Formen repräsentieren den normalen Strukturtyp zweisilbiger Wörter, in den Pot.-Formen haben wir wiederum erwartungsgemäss in der 2. Silbe den langen Kontraktionsvokal (s. E. Itkonen $M S F O u$ 88 S. 84, 91, 120-122). In der 2. Sg.Pot. der ungleichsilbigen und der $j e$-Verben steht ausser dem Potentialzeichen (b́D́ź, ’́ Kontraktionsvokal, während die Formen der 3. Pl. sekundär verkürzt sind (s. näher MSFOu 143 S. 112-113, 115-116). Der Unterschied in der Quantitätsstruktur zwischen der 1. Sg.Prs. und dem Part. Perf. bei den im Stamm dreisilbigen 
Verben geht zurück auf den unterschiedlichen Verlauf der Intensität dieser Formen, was wiederum auf der ursprünglich verschiedenen Silbenzahl beruht (s. S. 237). Der distinktive Faktor in der Opposition zwischen 1. Sg. Prs. und Parf.Perf. sowie Nomen actionis der Kontraktionsverben liegt in der Quantität des Kontraktionsvokals in der zweiten Silbe. Der Kontraktionsvokal der ersteren Form ist lang, da von den kontrahierten Vokalen der erstere betonter war ( $t^{\prime} s_{0 k} k^{\prime} \dot{d} m<$ *čŏkkajěm), der der letzteren Form dagegen kurz, da der letztere der kontrahierten Vokale betonter war (t'sokk'am< *čókkajèmè, -me) (s. E. Itkonen FUF 27 S. 161-162, MSFOu 88 S. 115).

Ausser dem Südlappischen ist dem Westlappischen auch jener Zug des Inarilappischen fremd, dass der Metaphoniewechsel der ersten Silbe morphologisch distinktiv geworden ist. Das trifft zwar nur auf einen Fall zu, und zwar auf die Opposition zwischen der 1 . Sg.Prät. und dem Nomen actionis bei den gleichsilbigen $e^{-}$und $o$-stämmigen Verben (und auch dem Part.Perf. der o-stämmigen Verben), unter der Voraussetzung, dass in der ersten Silbe ein etymologisch linger $\mathrm{V}_{0-}$ kal steht. Die dann in der 2. Silbe beim Nomen act. (bei den o-stämmigen Verben auch im Part.Perf.) auftretenden Vokale ${ }^{*} e$ und $*_{n}$ wurden zu $i$ und $u$. Da auch in der 2. Silbe der 1. Sg. Prät. $i$ und $u$ stehen (Kontraktionsvokale), bleibt die Opposition auf die Vokalqualität der 1. Silbe angewiesen, denn vor urspr. ${ }^{*} e$ und ${ }^{*} n$ hat sich eine andere Vokalqualität entwickelt als vor urspr. $i$ und $u\left(\bar{a} \breve{n} n u m--\bar{a} \breve{n} n u m<*^{*}\right.$ aǹum - *aǹnme, $-m \check{e})$. Die Opposition ist jedoch völlig neutralisiert in den $e$-stämmigen Verben, die in der 1 . Silbe einen Vertreter von urlp. ${ }^{*} \varepsilon$ oder ${ }^{*} e$ haben, denn ${ }^{*} \varepsilon>e$ und $*_{e}>i e$ sowohl vor einem $e$ als auch $i$ der 2. Silbe (s. E. Itkonen MSFOu 79 S. 42-43), z.B. 1. Sg.Prät. pett' $i m<{ }^{*}$ pettim - Akt. pett' $i m<$ *pètteme 'betrügen'.

Abweichend von den westlappischen Dialekten begegnet im Inarilappischen auch ein solcher Wechsel der Vokalquantität in morphologisch distinktiver Funktion, dessen eine Seite ein durch den Schwund der Verbindung * $n_{3} \zeta$ hervorgerufener Kontraktionsvokal ist (s. näher E. Itkonen $F U F 27$ 
S. $159-160$, MSFOu 88 S. 120-122). Derartige Kontraktionsvokale begegnen in den Paradigmen der mit $n_{3} z$ abgeleiteten Nomina und im Potentialis der gleichsilbigen Verben. In allen Fällen ist mit dem qualitativen ein quantitativer Wechsel verbunden, entweder der Stufenwechsel (1. Sg.Pot.

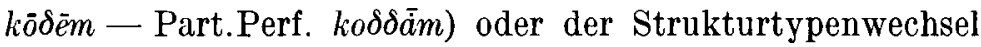
(1. Sg.Prs. polt'òm-1. Sg.Pot. poltt'ōm) oder beide (1. Sg. Prät. tśăllim - 1. Sg.Pot. tšālèm). Der durch den Schwund von ${ }^{*} n \check{z}$ bewirkte Kontraktionsvokal ist, wie diese Vokale im allgemeinen, in gewissen Fällen identisch mit einem anderen Grenzvokal. Auch dann können der Stufenwechsel, der Strukturtypenwechsel oder beide den distinktiven Faktor ausmachen (s. z.B. unter $\mathrm{S}(+\mathrm{T})$ und $\mathrm{T}$ ).

Auch im Paradigma der mit $\breve{a k}$ abgeleiteten Nomina begegnen Kontraktionsformen (s. E. Itkonen MSFOu $79 \mathrm{~S} .378$, $88 \mathrm{~S} .113-115$ ). Es ist nicht der einzige morphologisch distinktive Faktor, erscheint aber zusammen mit einem durch Überdehnung verursachten Wechsel und Stufenwechsel in dem Formpaar Nom.Sg. - Gen., Akk.Sg. (mie sts - mĭĕšstu, in Konsonantismen der Reihe $x x$ und $x y$ keine Überdehnung), wo der nichtkontrahierte Vokal mit dem Kontraktionsvokal wechselt.

Skoltlappisch, Dialekt von Suonikylä (E. Itkonen MSFOu 79 und 88 i.f. abgekürzt als E.I., eigene Aufzeichnungen)

$\mathrm{S}$

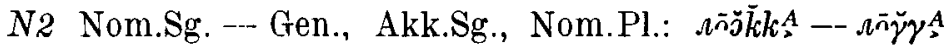

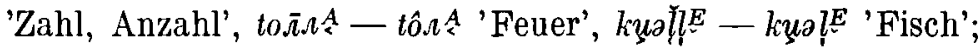

Nom.Sg. + 2., 3. Px - Gen., Akk.Sg., Nom.Pl. + 2., 3. Px: kus̀sà (E.I.) - kūžzą (E.I.) 'Kuh', kus̀sęz (E.I.) -

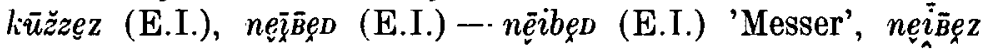
(E.I.) - nèibęz (E.I.).

N3 (in der 2. Silbe ein anderer Vokal als $e \sim a$ ) Nom. Sg. Gen., Akk.Sg., Nom.Pl.: kñ̄̆

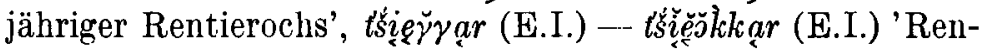
tierherde'.

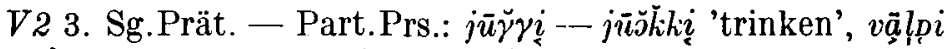

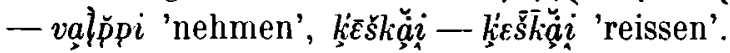


$V 2 e o$ 1. Sg.Prs. - Part.Perf. (V2o auch Nomen act.):

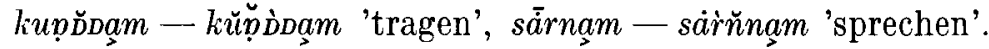

V2o 3. Sg.Prs. -- 2. Sg.Imp.: sär̆nna-särn $\vec{\sim}$.

N3 Nom.Sg. - Gen., Akk.Sg., Nom.Pl.: leə̆kkaš (E.I.) leăk̀kaž (E.I.) 'glücklich'.

$\mathrm{E}$

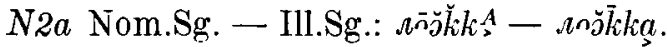

V2o 3. Sg.Prs. - 3. Pl.: ān̆nA - $\dot{a} \bar{n} n a$ 'bitten, flehen'.

M 1

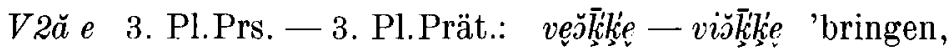

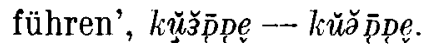

M 2

N2e Nom.Sg. + Px 2. Du.-Pl., Part.Sg. - Nom.Sg. + Px

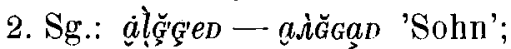

Nom., Gen., Akk.Sg., Nom.Pl. + Px 1., 2. Sg. - Nom., Gen., Akk. Sgr., Nom.Pl. + Px 1., 2. Du., Pl.: ajĭgam-

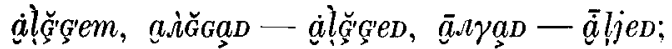

Gen., Akk.Sg., Nom.Pl. + Px 1. Du., Pl., Ess.Sg. - Gen.,

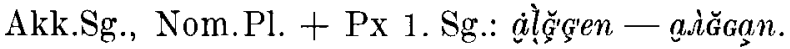

$V 2 e 3$ je Part.Perf. - Nomen act.: kŭọ̆̀̀ muš̄tsam - muš̄tlem 'erzählen', vell lijam - vell lijem 'springen'.

$\mathrm{C}$

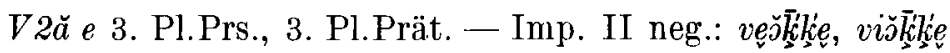

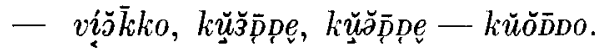

V20 3. Pl.Prs. -- 3. Pl.Prät., Imp. II neg.: särn̆na-saìnno.

$V 3$ je dit 1. Sg. Prs., Part.Perf. - 1. Sg.Prät. (Vdit auch Nomen act.): màinn stam-màin $^{A} s t e m$ 'Märchen erzählen',

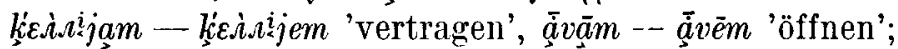

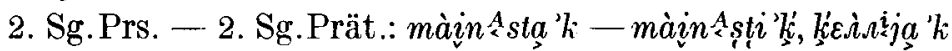

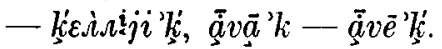

Vdit 3. Sg.Prs., 2. Sg.Imp. 一 Inf.: ạa vad, $\bar{a} v e D-\bar{a} v e \bar{D} D$.

$\mathrm{F}$

N2ă $e$ Gell., Akk.Sg., Nom.Pl. - Gen.Pl.: jōy̆yః (E.I.) $j \bar{o} \bar{\gamma} \gamma^{i}$ (E.I.) 'Fluss', pạạn (E.I.) - päni (E.I.) 'Zahn';

Gen., Akk.Sg., Nom.Pl. + 2. Px-Akk., Ill.Pl.: (Px 2.

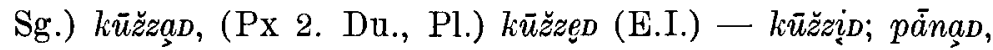
pạạned - päñid (E.I.). 


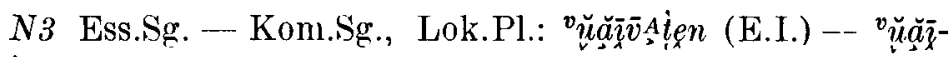
गैatin (E.I.) 'Knäuel';

Part.Sg. - Akk., Ill.Pl.: voǹn Așed - voǹn ş̦io 'Boot';

Nom., Gen., Akk.Sg., Nom.Pl. +- 2. Px. - Akk., Ill.Pl.:

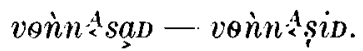

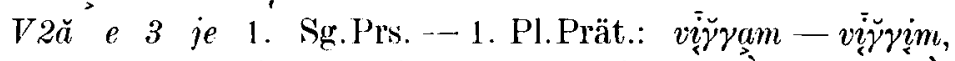

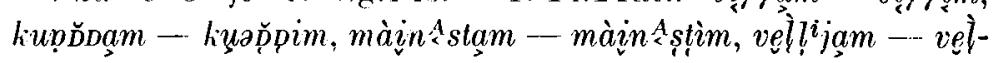
$l^{i}$ jim.

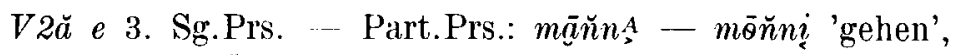
puaว̌t ${ }_{>}^{A}-$ puasttti 'kommen';

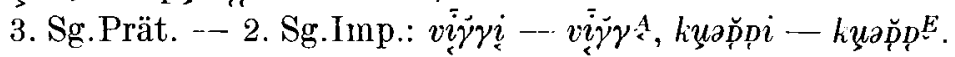

V3 je 1. Sg.Prs., Part.Perf., Nomen act. - 1. Pl.Prät.: màin Astam, -stem - màin Astim;

2. Pl.Prät. - Inf.: muš̃ $\delta$ ijed 'bleiben, verlassen werden'.

$\mathrm{S}+\mathrm{E}$

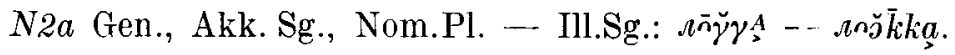

$V 2 e$ (in der 1 . Silbe ein anderer Vokal als $\breve{u}, e$ oder o), V2o 3. Pl.Prs. - 2.Sg.Imp.: pe $\bar{\delta} \delta e-p \bar{e} \delta^{E}$ 'schmelzen', $\dot{a} \bar{n} n a-\vec{a} n_{>}^{A}$.

$\mathrm{S}+\mathrm{M} 2$

N2e Nom.Sg. + Px. 2. Sg. -- Gen., Akk. Sg., Nom.Pl. + $\mathrm{Px}$ 2. Du., Pl.: aj̆ĞGaD - $\dot{a} l j e n ;$

N3e Nom.Sg. - Gen., Akk.Sg., Nom.Pl.: sūvęs (E.I.) sŏ̌̀ppaz (F.I.) 'Kiel des Bootes'.

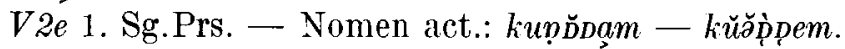

$\mathrm{S}+\mathrm{F}$

N2ă e Nom.Sg. - Gen.Pl.: jō̌kl

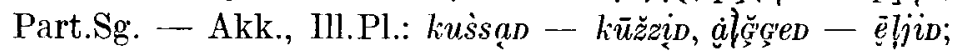

Nom.Sg. + 2. Px-Akk., Ill.Pl.: (Px 2. Sg.) kussad, (Px

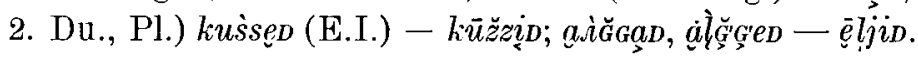

N2ă Ess.Sg.- Kom.Sg., Lok.Pl.: kus̉sąn - kiūzzżn.

N2e Ess.Sg.; Gen., Akk.Sg., Nom.Pl. + Px 1. Du., Pl. --. Kom.Sg., Lok.Pl.: pāăñnen-pānin.

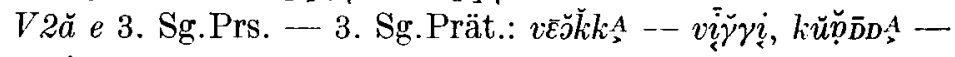

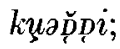

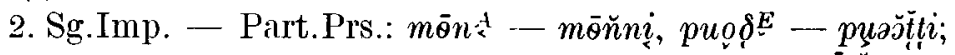

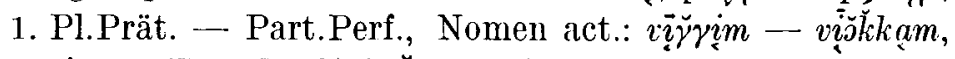

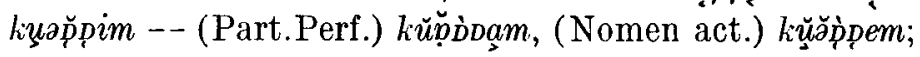


2. Pl.Prät. - 2. Pl.Imp., Inf.: viุ kưăpุped.

$\mathrm{S}+\mathrm{E}+\mathrm{M} 1$

V2e 3. Pl.Prät. - 2. Sg.Imp.: pưăšțe - puod $\delta^{E}$.

$\mathrm{S}+\mathrm{E}+\mathrm{C}$

N2ă e o Gen., Akk.Sg., Nom.Pl. - Ill.Sg.: muora - mŭăr̆ re

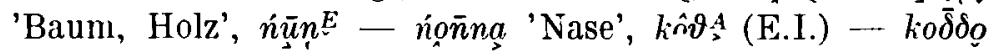
(E.I.) 'Laich'.

Nje Nom.Sg. - Gen., Akk.Sg., Nom.Pl.: sup̣.s. (E.I.) sǔŏiso (E.I.) 'Insel'.

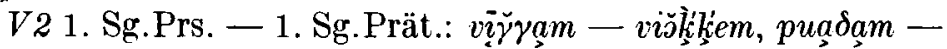
pŭăòttem, ảnam - à̀nom;

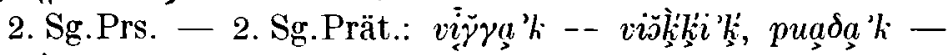
pŭăŏțti 'kì, ána' $k$ - aǹno' $k$;

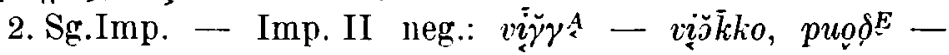

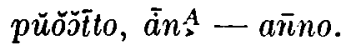

V2ă 3. Pl.Prs., 3. Pl.Prät. - 2. Sg.Imp.: veojhke, viokilie vījys.

V2o 3. Pl.Prät. - 2. Sg.Imp.: añno- $\bar{a} n_{s}^{A}$.

$\mathrm{S}+\mathrm{C}+\mathrm{F}$

N2ă $e$ Ess.Sg. - Akk., Ill.Pl. + 1. Px: no d̀ $\delta a n-(\mathrm{Px} 1 . \mathrm{Sg}$.

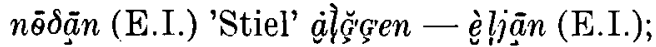

Gen., Akk.Sg., Nom.Pl. + 1. Px. - Akk., Ill.Pl. + 1. Px:

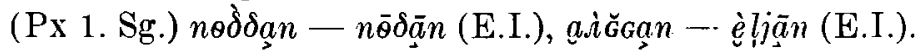

$\mathrm{S}+\mathrm{E}+\mathrm{C}+\mathrm{F}$

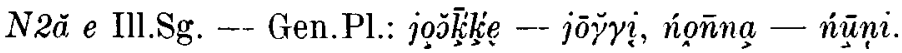

Nje Nom.Sg. -- Gen.Pl.: sup $\wedge_{A}$ (E.I.) - (selten) sŭŏsiıi (E.I.).

V2ă e 3. Pl.Prs., 3. PI.Prät., Imp. II neg. - 3. Sg.Prät.:

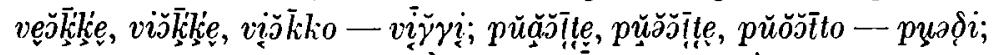

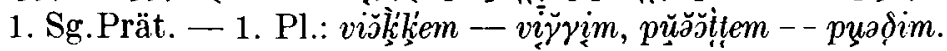
$\mathrm{E}+\mathrm{C}$

V2ă e 3. Sg.Prs. - 3. Pl.Prs., 3. Pl.Prät., Imp. II neg.: mān̆n

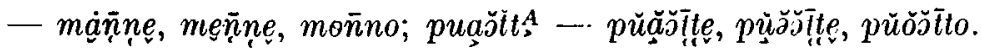
V2o 3. Sg.Prs. - 3. Pl.Prät., Imp. II neg.: än̆ns - añno.

V2ă o 1. Sg.Prät. - Part.Perf., Nomen act.: juŏkgkem jūŏkkạm, à̀nom - àn̆nam.

V2e 1. Sg.Prät. - Part.Perf.: pŭăŏttem - puẵ̆tam. 
$\mathrm{E}+\mathrm{C}+\mathrm{F}$

Năk Nom.Sg. - Gen., Akk.Sg., Nom. Pl.: kāămmį (E.I.) kā̄me (E.I.) 'Schuh'.

V2ă e 3. Pl.Prs., 3. Pl.Prät., Imp. II neg. - Part.Prs.: veškske,

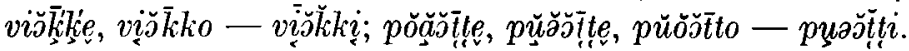

$\mathrm{C}+\mathrm{F}$

N3 Ill.Sg. - Gen.P1.: voǹn ${ }^{A} s a-v \theta \grave{n} n^{A} s i$

Lok.Pl. - Akk., Ill.Pl. + Px 1. Sg.: voìn $n^{A} \sin -v \theta n \cdot n<\operatorname{sān}$ (E.I.).

Nje Gen., Akk.Sg., Nom.Pl. - Gen.Pl.: sǔŏsiso (E.I.) (selten) sŭŏdıi (E.I.).

$V 3$ je dit 1. Sg.Prät. -- 1. Pl.: màin Asstem - màincstìn,

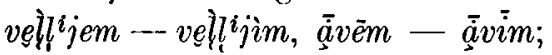

3. Pl.Prs., 3. Pl.Prät. - 3. Sg.Prät.: muštzle -- mušț li,

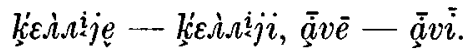

$V$ dit 1. Sg.Prs., Part.Perf. - 1. Pl.Prät.: ạa $\bar{a} m-\bar{a} v i m$; ävin.

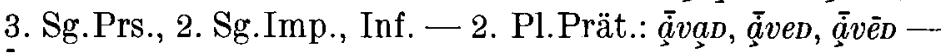

$\mathrm{A}$

N2ă Nom., Gen., Akk.Sg., Nom.Pl. + Px 1., 2. Sg - Nom., Gen., Akk.Sg., Nom.Pl. + Px 1., 2. Du., Pl.: kus̀sam (E.I.) kuśsem (E.I.), kus̀sad (E.I.) - kus̀sẹp (E.I.), kussan (E.I.) kussen (E.I.), kūžzaad (E.I.) - kūžzę (E.I.);

Ess.Sg. - Gen., Akk.Sg., Nom.Pl. + Px 1. Sg. : kus̀sąn-kus̀san (E.I.).

PPr. Nom.Sg. - Gen., Akk.Sg.: mon - $m \bar{u}$ 'ich'.

$V 3$ dit 3. Sg.Prs. - 2. Sg.Imp.: māinast - mäinąst, àa vă - $\bar{a} v e D$.

Das Skoltlappische hat mehr morphologisch distinktive Lautwechsel im Innern des Stammes als irgendein anderer der hier behandelten Dialekte und wahrscheinlich mehr als überhaupt eine lappische Mundart. Und das ungeachtet dessen, dass der untersuchte skoltlappische Dialekt, der von Suonikylä, den Dual in der Konjugation und in der possessiven Deklination verloren hat, wodurch die Anzahl der paradigmatischen Oppositionen beträchtlich abgenommen hat.

Der Reichtum der morphologischen Funktionen der Wechsel im Stamminnern beruht im Skoltlappischen entscheidend 
auf bestimmten Vorgängen von Kontraktion und Verschme]zung der Vokale, wie sie in den anderen Dialekten nicht bekannt sind. Das an der Grenze zwischen der 2. und 3. Silbe und weiter entfernt im Wort zwischen Vokalen stehende $D$ ist geschwunden und die auf diese Weise nebeneinander gelangten Vokale haben einen Kontraktionsvokal gebildet (s. E. Itkonen MSFOu 79 S. $380-382,88$ S. 194-196, 207). Eine Folgeerscheinung dieser Kontraktion ist die morphologische Distinktivität des im Paradigma der mit dit abgeleiteten Verben auftretenden Wechsels der verschiedenen Kontraktionsvokale (1. Sg.Prs. $\bar{\alpha} v a \bar{a} m--1$. Sg.Prät. $\bar{a} v e \bar{e} m<{ }^{*} \bar{a} v^{e}-$ Dam - * $\bar{a} v^{e}$ pem usw., 1. Sg. Prät. doppelte Kontraktion ${ }^{*} \bar{a} v^{e}-$ pem < *aveDějĕm). In der 3. Sg.Prs. und der 2. Sg.Imp. hat sich keine Kontraktion vollzogen, so dass diese Formen durch einen Wechsel von nichtkontrahiertem und kontra-

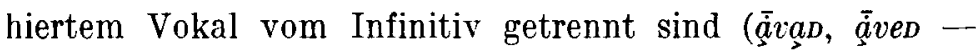
åvẽs). In der Flexion der ăk-Nomina begegnet ein auf ähnlicher Kontraktion beruhender Wechsel wie auch im Inarilappischen, mit dem Unterschied, dass im Skoltlappischen der Nom.Sg. in der starken Stufe steht und im Auslaut einen Verschmelzungsvokal hat $\left(k \bar{a} \bar{m} m i<{ }^{*} k \bar{a} \bar{m} m a j-<{ }^{*} k \bar{a} \bar{m} m a G^{\prime}-\right)$ (vgl. F. Itkonen MSFOu 79 S. 384).

Die im Skoltlappischen in der hauptbetonten Silbe eingetretenen Veränderungen ${ }^{*} \check{a} j>i$ und ${ }^{*} i j>i$ (s. a.a.o. 383-386) haben eine ganze Reihe von Fusionen bewirkt, wo das suffixale ${ }^{*} j$ und der vorangehende Auslautvokal des Stammes einen Verschmelzungsvokal gebildet haben. Der Wechsel zwischen ihm und dem nicht verschmolzenen Auslautvokal des Stammes ist in zahlreichen Fällen morphologisch distinktiv. Das fusionierte ${ }^{*} j$ war dann das Zeichen des Pl., des Prät. oder des Part.Prs. (Gen.Pl. jō $\breve{\gamma} \gamma \underline{i}<{ }^{*} j$ jŏGĕj,

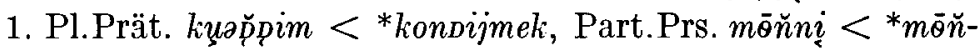
nai $<{ }^{*}$ mènèje). Schon die hohe Frequenz dieser Morpheme bewirkt, dass es auch viele durch den Verschmelzungsvokal hervorgerufene morphologisch distinktive Wechsel gibt.

Das Skoltlappische ist der einzige Dialekt, der den qualitativen Stufenwechsel der Sibilanten an der Grenze zwischen 2. und 3. Silbe erhalten hat, wie er zu Beginn dieses Jahrhun- 
derts auch noch im Inarilappischen auftrat (s. E. Itkonen FUF 27 S. 145-148). Als einziger morphologisch distinktiver Faktor tritt diese Art des Stufenwechsels nur in der Flexion solcher im Stamm dreisilbiger, auf $s$ und $s$ endenden Nomina auf, deren Konsonantismus an der Grenze zwischen 1. und 2. Silbe nicht dem Stufenwechsel unterliegt (leăkłkašs - leăkkaž $)$.

Durch den Schwund der Endung $n$ des Ill.Sg. wurde die Quantität zum einzig unterscheidenden Faktor zwischen dem Nom.Sg. und dem Ill.Sg. der zweisilbigen $a$-stämmigen

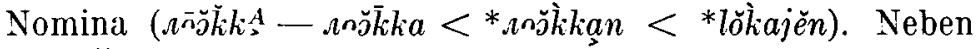
der Überdehnung ist die unterschiedliche Quantität des Auslautvokals distinktiv. Mit der überkurzen Vokalquantität ist ferner Stimmlosigkeit verbunden.

Wegen des Zusammenfallens von $*_{i}$ und $*_{\boldsymbol{e}}$ in der unbetonten Silbe hat die Metaphonie eine morphologisch distinktive Funktion zwischen dem Prs. und dem Prät. in der 3. Pl. der $\breve{a}$ - und $e$-stämmigen gleichsilbigen Verben (vešlikge viōkike usw.).

Von den Fällen, wo mehrere Arten von Wechseln im Stamminnern gleichzeitig morphologisch distinktiv sind, unterscheiden sich die folgenden Gruppen als typisch für das Skoltlappische, wo der Wechsel jeweils durch Kontraktion und Verschmelzung verursacht wurde: $\mathrm{S}+\mathrm{C}+\mathrm{F}, \mathrm{S}+\mathrm{E}+\mathrm{C}+$ $\mathrm{F}, \mathrm{E}+\mathrm{C}+\mathrm{F}$ und $\mathrm{C}+\mathrm{F}$. Kontraktion und Verschmelzung können auch in ein und derselben Form begegnen, z.B. Akk.,

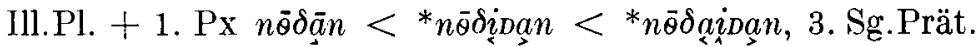
$\bar{a} v \bar{i}<{ }^{*} \dot{a} v^{e} p i<{ }^{*} \overrightarrow{\dot{a}} v^{e}$ pii (vgl. E.Itkonen MSFOu 79 S. 381);

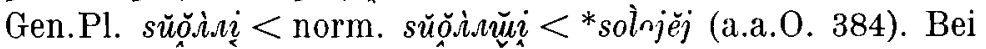
dem grössten Teil der Fälle, wo Kontraktion und Verschmelzung zusammen den Vokalwechsel bewirkt haben, steht in der einen Form des Wechselpaares der Kontraktionsvokal und in der anderen der Verschmelzingsvokal, z.B. Ill.Sg.

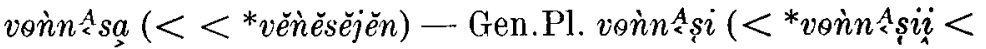
*věnĕš̆j).

In Gruppe A (= sonstige qualitative Wechsel) beruhen die unregelmässigen $a$ - und $e$-Vokale in einigen Formen der possessiven Deklination von gleichsilbigen $\breve{a}$-stämmigen Nomina, 
die einen paradigmatischen Wechsel verursachen (kussam kuśsem, kussan - kuśsan usw.), auf der Analogie zu den $e^{-}$ stämmigen Nomina (s. a.a.O. 96-98).

Aus dem oben Dargestellten ging hervor, dass ron den Wechseln im Innern des Stammes der Stufenwechsel und die damit verbundenen quantitativen Wechsel am häufigsten für die Flexion distinktiv auftreten. Im Südlappischen fehlt der Stufenwechsel und es gibt dort auch am wenigsten morphologisch distinktiven Lautwechsel im Stamminnern. Schon früher (S. 224, 251) wurde festgestellt, dass sich das Südlappische hinsichtlich seines geringen Symbolismus stark von den übrigen lappischen Mundarten unterscheidet. Andererseits ist für das Südlappische charakteristisch, dass sich die Auslautlaute des Wortes gut erhalten haben. Die ältesten nach dem Urlappischen eingetretenen Schwundfälle eines Auslautes betreffen alle anderen Dialekte ausser dem südund Umelappischen. Und doch dürfte das nicht ohne weiteres so ausgelegt werden, dass die Schwunderscheinungen im Norden des Umelappischen zurückgingen auf irgendeine "ost-nordlappische" Ursprachenform, von der sich das Südlappische schon getrennt gehabt hätte. In den Dialekten, wo der Stufenwechsel systematisch alle Konsonantismen nach der betonten Silbe betrifft, konnten auslautende Vokale und Konsonanten auch ohne eine direkte Verbindung zwischen den Dialekten verschiedenerseits ausfallen. Die einzelnen Mundarten haben unabhängig voneinander die Eigenschaft des Stufenwechsels, den offenen oder geschlossenen Charakter der nachfolgenden Silbe anzuzeigen, ausnutzen können. So schwand in zahlreichen Fällen der Auslautkonsonant eines zweisilbigen, auf Konsonanten endenden Wortes, ohne dass eine störende Homonymie entstand, denn die schwache Stufe trennte ein urpr. auf Konsonanten endendes von einem urspr. auf Vokal ausgehenden zweisilbigen Wort, dessen Stammkonsonantismus die starke Stufe aufweist. Entsprechend hat die in einem dreisilbigen auf Vokal endenden Wort eingetretene Apokope keine Vermischung zustande kommen las- 
sen mit den urspr. auf Konsonanten endenden zweisilbigen Wörtern. Der Schwund der Endung $n$ des Gen.Sg. z.B., der sehr konsequent in allen Dialekten ausser dem Süd- und Umelappischen zum Ausdruck kommt, braucht durchaus nicht dasselbe zu sein wie die auf den Urdialekt zurückgehende Erscheinung. Bei den meisten Substantiven, nämlich bei den im Stamm gleichsilbigen und bei den ungleichsilbigen, deren Nom.Sg. auf einen Konsonanten endete, unterschied der Stufenwechsel den Nom. vom Gen.Sg., wodurch in allen Stufenwechseldialekten die gleichen Voraussetzungen vorhanden waren für den Schwund von $n$ (urlp. ${ }^{*}$ kole $:{ }^{*}$ kolen $>$ lp N guolle: guole 'Fisch', urlp. *nĕger : nĕkarèn $>\operatorname{lp~N}$ nâger : nâkkar 'Schlaf'). Dass der Schwund des $n$ des Gen.Sg. in einigen Dialekten eine relativ junge Erscheinung ist, geht aus jenen vielen Angaben aus dem letzten Jahrhundert hervor, wonach im Pite- und Lulelappischen sowie dialektweise im Norwegischlappischen unter wechselnden Voraussetzungen auf - $n$ endende Formen des Gen.Sg. üblich waren (s. Halász, Pite lappmarki szótár és nyelvtan, Budapest 1896, S. XV, XVII, XX-XXI; Wiklund, Laut- und Formenlehre der Lule-Lappischen Dialekte, Stockholm 1891, S. 176, Festskrift til Rektor J. Qvigstad, Oslo 1928, S. 327; Ravila MSFOu 62 S. 92-93). Die Apokope von Vokalen in der dritten oder einer entfernteren Silbe ist offenbar älter als die meisten Fälle von Konsonantenschwund im Auslaut, denn sie hat sich ausserhalb des Süd- und Umelappischen sehr regelmässig vollzogen. Der Ess.Sg. z.B. lautet überall ausser im Süd- und Umelappischen auf $-n$ aus $\left(<^{*}-n e<{ }^{*}-n a\right)$. Wahrscheinlich gab es auch in einigen anderen Westdialekten als dem Pitelappiscben einmal eine Phase, wo sowohl im Gen. als auch im Ess. Sg. die Endung $n$ auftrat, vgl. LpPi Gen.Sg. tålōn : Ess.Sg. tållon 'Feuer' (Halász a.a.O. XVII). Irgendeinen Unterschied zwischen diesen Endungen muss es jedoch gegeben haben. Im Pitelappischen wechselt die Schwundstufe mit dem $n$ im Gen.Sg. Offenbar war das urspr. auslautende $n$ des Gen. schon geschwächt, wenn nicht sogar unter bestimmten Bedingungen schon geschwunden, als im Essiv Apokope eintrat. Später schwand dann in den einzelnen Dialekten speziell das geschwächte oder mit $\emptyset$ wechselnde $n$. 
Die schwache Stufe wurde auch zum Charakteristikum einiger urspr. auf $-k$ endenden Formen, in denen der Auslautkonsonant geschwunden ist. Dies trat schon sehr frühzeitig in einigen längeren Suffixen ein, in denen das $-k$ keine lebendige Funktion besass. Es ist durchaus begründet, den Schwund des angenommenen Auslaut- $k$ in der Endung der 2. Pl.Prs., Pot. und Imp. zurückzuführen bis ins Urlappische (vgl. 1pSd. 2. Pl.Prs. boàtaŕ, N boattebēttit, Kld. puaptippț 'kommen', s. ausführlicher MSFOu $143 \mathrm{~S}$. 327-329). Als relativ alt hat auch der Schwund des Auslaut-k des Infinitivzeichens zu gelten, der alle anderen Dialekte betrifft ausser dem Südlappischen, schon aus dem Grund, weil der infolge des Schwunds von $-k$ in den Auslaut gelangte Vokal überall von der Apokope mit erreicht wurde, vgl. lpSd. boåtzjh $\sim$ U bååhteet ${ }^{1}$ (Schlachter), $\mathrm{N}$ boattet, Kld. puaptc $(\delta)<{ }^{*}$ pritedek. Ferner ist das $-k$ in den zweisilbigen, mit $k$ abgeleiteten Nomina anderswo ausser im Südlappischen geschwunden $(\operatorname{lp} \mathrm{N} b \bar{a} n \hat{a}: b \bar{a} d n \hat{a} g(\hat{a})$ 'Hund' usw.), wo es auch keine eindeutige Funktion besitzt. Sonst hat sich das $(-k>)-h$ im Schwedischlappischen erhalten, wenn auch schwach und mit der Schwundstufe wechselnd, während im Norwegisch- und Ostlappischen die Erhaltung des $-k$ abhing von seiner Funktion. Je weiter östlich wir kommen, auf desto mehr Schwundfälle treffen wir. Teilweise ist das dadurch verständlich, dass die symbolischen Ausdrucksmöglichkeiten in den östlichen Dialekten besser

${ }^{1}$ Der Schwund des Infinitiv- - $k$ im Umelappischen braucht nicht in Verbindung zu stehen mit der entsprechenden Erscheinung in den nördlicheren Dialekten, sondern es scheint sich dabei eher um den Schwund eines kurzen unbetonten Vokals vor $h\left(<{ }^{*} k\right)$ zu handeln, wie er auch in einigen anderen Fällen auftritt und wodurch dann das $h$ mit dem vor dem Vokal stehenden Konsonanten verschmolz, z.B. Nom.Pl. juhk (<*juhkh < *juhkah<*juhkak) vom Wort juhka 'Fluss' (vgl. gied'geeh vom Wort geäd'gee 'Stein'), gàbmas (<*gàbmash < *gabmasah < *gabmasak) vom Wort gaamas 'Renbeinhaut' (vgl. bàlgaah vom Wort baalgès 'Weg'); 3. Pl.Prs. büüht (<*büühth < *büühtah) vom Verb bååhteet 'kommen' usw. (s. Schlachter, Wörterbuch des Waldlappendialekts von Malå, Helsinki 1958, S. XIV-XIX). Ebenso vielleicht Inf. bååhteet $<{ }^{*}$ bååhteeth $<{ }^{*}$ bååhteetah. Die Erscheinung dürfte historisch zusammenhängen mit dem im Südlappischen auftretenden Schwund eines a vor einem $h \mathrm{im}$ Wortauslaut, vgl. Rör. Inf. boålajh < *boàtajah (Bergsland, Røros-lappisk grammatikk S. 66-67, 74-75). 
sind. So konnte das $-k$ z.B. in der 3. Pl.Prs. der im Stamm gleichsilbigen Verben des Russischlappischen schwinden, ohne dass die Form identisch geworden wäre mit der 3. Pl. Prät., in der das $-n$ schon früher geschwunden war, wenn der Metaphoniewechsel der 1. Silbe phonologisch ist, z.B. lpSk. 3. Pl.Prs. mänñe: 3. Pl.Prät. meñne < ${ }^{*}$ màñnek : meñne < *mĕǹnek: *měñnin 'gehen', vgl. lp N mânnik : mânni. In der 3. Pl.Prs. der dreisilbigen und der Kontraktionsverben ist das -k auch geschwunden, obgleich diese Verben nicht die Möglichkeit haben, die Formen der 3. Pl.Prs. und Prät. auseinanderzuhalten (z.B. lpSk. 3. Pl.Prs., Prät. muš̄lle $\sim$ N Prs. mui'tâlik : Prät. mui'tâleqgji 'erzählen'). Das Muster der gleichsilbigen Verben verursachte offenbar auch den Schwund von $-k$ in den dreisilbigen und den Kontraktionsverben. $\mathrm{Zu}$ beachten ist, dass die Frequenz des Auftretens dieser Verben viel kleiner ist als die der gleichsilbigen. Von den 611 Verbformen z.B., die auf den Seiten 163-175 der in der Sammlung von T. I. Itkonen "Koltan- ja kuolanlappalaisia satuja" ( $M S F O u$ 60) veröffentlichten Texte im Dialekt von Suonikylä stehen, sind nur $17 \%$ im Stamm dreisilbig und Kontraktionsverben. Und doch hat die Fülle der Schwundfälle von $-k$ im Russischlappischen auch eine systematischere Neutralisierung der Oppositionen verursacht, i.a. jedoch nur da, wo die Distribution der Glieder so unterschiedlich ist, dass die Funktion der Formen aus dem Satzzusammenhang hervorgeht. Der Schwund des $-k$ als Zeichen des Nom.Pl. der Substantive z.B. machte im Russischlappischen diese Form dem Gen. und Akk.Sg. gleich (z.B. lpSk. Gen., Akk.Sg., Nom.Pl. tô.sa $\sim \mathrm{N}$ Gen., Akk.Sg. dolâ: Nom.Pl. dolâk 'Feuer'), die Distribution der Formen ist jedoch so unterschiedlich, dass durch die Gleichheit nur äusserst selten Verwirrung gestiftet wird.

Der Schwund der Numeruszeichen $-n$ und $-k$ in den Px des Pl. und Du. und in den Formen der 1. und 2. Du. und Pl.Prät. und Kond. im Ostlappischen hat verursacht, dass die Opposition zwischen in gleichen Satzstellungen auftretenden Formen neutralisiert wurde. Im Inarilappischen hat sich die Opposition in der Flexion der Verben so erhalten, dass der durch die zur Personalendung gehörende Vokalqualität be- 
dingte, im Präteritumzeichen erscheinende Wechsel $i \sim i$ nunmehr den Numerusunterschied auszudrücken hatte, z.B. 1. Du.Prät. politt’òim, 2. -D (<* pölt̀njmèn, *-pĕn), 1. Pl. politt'ò̀m, 2. -D (<* ${ }^{*}$ oŏltinjmek, *-Dek) 'drehen, durchwühlen'. Die Opposition kann auch im Auslautvokal des Stammes oder im Sekundärstamm des Konditionalis zum Ausdruck kom-

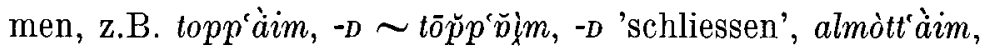
$-D \sim$ almòtt'rìm, $-D$ 'melden, mitteilen', làim, $-D \sim$ leìm, $-D$

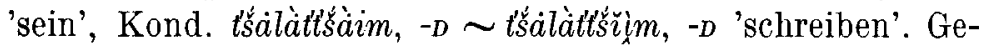
nauer über den Wechsel s. Collinder, Über den finnisch-lappischen Quantitätswechsel S. 140-145, 189-191; E. Itkonen MSFOu 79 S. 86-95. In der possessiven Flexion hat das Inarilappische den Unterschied zwischen Du. und Pl. nicht erhalten und im Russischlappischen sind der Du. und der Pl. in der possessiven Flexion wie auch in der 1. und 2. P.Prät. und Kond. völlig zusammengefallen, ohne dass die Opposition auf den Stamm oder irgendein anderes Suffix übergegangen wäre. Es ist jedoch anzumerken, dass die funktionale Opposition der Dual- und Pluralformen - obwohl diese in gleicher syntaktischer Umgebung auftreten - sehr selten wirklich bedeutend ist. Hierauf wird es wohl zurückzuführen sein, dass der Dual in der possessiven Deklination und Konjugation in den anderen russischlappischen Dialekten ausser in Paatsjoki völlig hat schwinden können und dass der Dual überhaupt im Lappischen wie auch dialektweise in den obugrischen und samojedischen Sprachen eine zurückgehende Kategorie ist. (Tauli, Structural Tendencies in Uralic Languages, Uralic and Altaic Series 17, The Hague, S. 151-154.) Dass im Ostseefinnischen und Ungarischen Reste eines ehemaligen Duals festgestellt wurden (E. Itkonen Vir. $1955 \mathrm{~S}$. 171-; Bouda JSFOu 47,2 S. 53; Hajdú, Bevezetés az uráli nyelvtudományba, Budapest 1966, S. 131), scheint zu beweisen, dass das Zurückweichen des Duals in den uralischen Sprachen eine allgemeinere Tendenz darstellt. Das gleiche ist auch für die indoeuropäischen Sprachen festgestellt worden (Wackernagel, Vorlesungen über Syntax I, 2. Aufl., Basel 1950, S. 73---; E. Itkonen, Kieli ja sen tutkimus, Helsinki 1966, S. 253). 
Auch der Unterschied zwischen den singularischen und pluralischen Possessivsuffixen gleicht sich im Ostlappischen allmählich aus. Der Schwund des $-k$ und des ihm vorangehenden Vokals hat jedoch die Differenz nicht überall abgeschafft. In der 3. P. wurde der Stufenwechsel des urspr. nur auf die Person hinweisenden $s$ zum Ausdruck des Numerus (Snk. Nom. Pl. + Px 3. Sg. èmes - Px 3. Pl. èmęz 'Schwägerin') und die Opposition zwischen den Numeri der 1. und 2. P. der $e$-stämmigen Nomini wurde nunmehr durch den Metaphoniewechsel des Grenzvokals ausgedrückt (Snk. Nom.Sg. + Px 1.

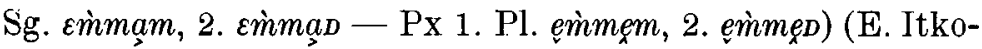
nen Vir. 1955 S. 166). Im Inarilappischen kamen die mit singularischen Possessivsuffixen versehenen Formen später an die Stelle der mit pluralischen und dualischen $\mathrm{Px}$ ausgerüsteten Formen (a.a.O.), aber wenigstens im skoltlappischen Dialekt von Suonikylä ist die symbolische Methode in den Bereich der $\breve{a}$-stämmigen Nomina eingedrungen, indem die mit dem Px der 1. und 2. Du. und Pl. versehenen Formen als Grenzvokal analog ein $e$ erhielten, während in den entsprechenden singularischen Formen ein $\underset{z}{a}$ steht (s. S. 274, 276-277).

Der einzige Fall, wo ein ursprünglich auslautendes $k$ in der nichtersten Silbe in allen Dialekten vom Südlappischen bis zum Terlappischen den Schwund vermieden hat, ist die Konjugationsform der 2. Sg. Ind., Pot. und Kond. Dies wie auch die Tatsache, dass ein urspr. auslautendes $m$ in der nichtersten Silbe den Schwund im Norwegisch- und Ostlappischen nur als Endung der 1. Sg.Ind., Pot. und Kond. vermieden hat, beruht darauf, dass die Unterscheidung dieser in gleichen syntaktischen Zusammenhängen auftretenden Formen von erstrangiger Wichtigkeit ist und es zwischen ihnen keinen im Stamm verankerten Unterschied gibt, der das Suffix hätte ersetzen können (s. MSFOu 143 S. 224, 227). Obgleich das $-m$ der 1. P.Sg. im Kolalappischen zumindest dialektweise erst kürzlich geschwunden ist, wird die Opposition doch weiter durch das $-k$ der 2 . P.Sg. oder seine Vertretung $-\chi$ ausgedrückt: Trb.

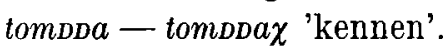

Ausser $n, m$ und $k$ ist auch das $j$ in einigen Fällen im Wortauslaut geschwunden. Das ist an sich auch bei der Vokalver- 
schmelzung geschehen (s.S. 45-46), doch begegnen im Norwegisch- und Russischlappischen auch Fälle, wo - $j$ geschwunden und doch nicht mit dem vorangehenden Vokal verschmolzen ist. Im lulelappischen Dialekt von Nord-Gällivare, im Norwegischlappischen sowie im Skolt- und Kildinlappischen ist das - $j$ im Nominativ der uje-Nomina geschwunden, z.B. IpL $\mathrm{NG}$ (Grundström) sướl̄̄, lp N suolo, lpSk. (E. Itkonen) sup̣ $\Omega A$, lp Kld. (T. I. Itkonen) şəə⿻コ一 $\left(^{(a)}\right.$ 'Insel'. In diesem ziemlich unproduktiven Ableitungstypus hat das - $j$ weder eine grammatische noch eine lexikalische Funktion.

Neben dem Schwund der Schlusslaute eines Wortes war es auch die Kontraktion, die eine Verdunklung der phonetischen Voraussetzungen des Stufenwechsels verursacht hat. In *mèǹim > lp N mânnim z.B., der Fortsetzung der urlp. Form der 1. Sg.Prät. *mënèjèm, gelangte die starke Stufe nach der Kontraktion vor eine geschlossene Silbe. Das ist relativ regelmässig geschehen, denn -- wie bereits K. B. Wiklund nachwies - die Kontraktion ist ursprünglich speziell vor einer geschlossenen Silbe anzunehmen (MO 9 S. 195). Durch diesen Umstand wurden eine Reihe von Belegen bewirkt, wo die morphologische Opposition ausgedrückt wird durch den Stu-

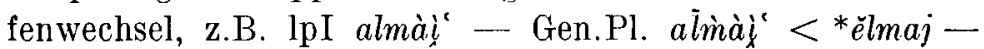
ëlmajëj 'Mann'. Meist verband sich mit diesen Fällen auch der Schwund der Schlusslaute des Wortes, z.B. IpL 2. Sg.Imp. pòntē -- 2. Du. pònhtē < * propek - *priteden 'kommen'. Ferner konnte die morphologische Funktion des durch Kontraktion eingebüssten Suffixelementes auf einen üherdehnten Stammkonsonantismus übergehen, z.B. $1 \mathrm{pN}$ 3. Sg.Prs. divvo - 1., 2. Du.Imp., Part.Prs. div'vo < ${ }^{*} t \grave{i} \dot{v}^{\prime}-{ }^{*} t i \grave{v} n j e n,{ }^{*}-$ Den, *-je 'reparieren'. Dies ging jedoch nicht, wenn der Stammkonsonantismus zur Reihe $x x$ oder $x y$ gehörte (suop'po suop'po 'werfen' usw.).

Die morphologischen Funktionen der durch Kontraktion geschwundenen Suffixe sind in der Hauptsache auf die Kontraktionsvokale übergegangen. Man muss die Entwicklung vom Frühurlappischen an verfolgen. Der Vokalismus der zweiten und weiter entfernten Silbe im Frühurlp. hatte wahrscheinlich die drei Phoneme ${ }^{*} a,{ }^{*} n$ und ${ }^{*} \check{e}$. Vor den urlp. Kontraktionen 
wurde ${ }^{*} a>e$ in der zweiten Silbe, wenn die Silbe die letzte im Wort war oder wenn in der dritten Silbe der Vertreter eines offenen Vokals stand, und offenbar auch $*_{n}>\breve{u}$, wenn sich in der dritten Silbe ein enger oder halbenger Vokal befand. Erkki Itkonen ist zwar in seiner Arbeit "Der ostlappische Vokalismus vom qualitativen Standpunkt aus" zu dem Ergebnis gekommen, dass in der zweiten Silbe der Wandel $*_{n}>\check{u}$ nicht im Urlp. eintrat, sondern im Westlappischen, nachdem sich das Ostlappische bereits vom Urlp. getrennt hatte ( $M S F O u$ 79 S. 113-114). Das ist auch das wahrscheinlichste Resultat, wenn die Vertretung der nichtkontrahierten Labialvokale vor allem im Inari- und Skoltlappischen untersucht wird. Die Qualität der Kontraktionsvokale aber und bestimmte Vokalverhältnisse im Kolalappischen scheinen in eine andere Richtung zu weisen, danach ist sowohl $*_{a}>e$ als auch $*_{n}>\check{u}$ schon im Urlappischen geschehen. Wie Erkki Itkonen nachgewiesen hat, ergab ${ }^{*} a+{ }^{*} \check{e}$ in der Kontraktion ein anderes Ergebnis als ${ }^{*} e+{ }^{*} e^{1}\left(<{ }^{*} a+{ }^{*} a\right)$ : aus ersterem wurde ${ }^{*} a$, aus letzterem *e (MSFOu 79 S. $315-319,324-329$ ), z.B. lp N Gen.Sg. âl'bma <*ĕlmajĕn 'Mann', sâr'va <* sĕrvazĕn 'Rentierbock' (Nom.Sg. âlmai, sârves) Part.Prs. diet'te < *tèteje < *tetaja 'wissen' (Inf. diettet). In gleicher Weise ergab die Kontraktion ${ }^{*} n+{ }^{*} e\left(<{ }^{*} n+{ }^{*} a\right)$ ein anderes Ergebnis als $*^{*} \check{u}+*_{\breve{e}}\left(<*_{n}+*_{\breve{e}}\right)$. Im vorhergehenden Fall weisen alle Dialekte auf den urlp. Kontraktionsvokal $*_{n}$, im letzteren zeigen alle Dialekte, auch die ostlappischen, in denen keine Anzeichen für ein nichtkontrahiertes ${ }^{*} u$ vorhanden sind, dass der urlp. Kontraktionsvokal $u$ war. Z.B.

LpSd. Rör. (Bergsland) Kontraktions- ${ }^{*}$ : 3 3. Pl.Prs. dajtah 'machen' (<*-njek); Kontr. -*u: 1. Du.Pers.-Pron. Gen.

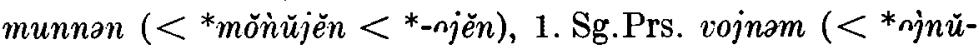

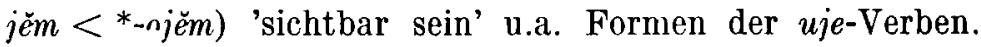

1 Offenbar wurde auch in der 3. Silbe frühurlp. ${ }^{*} a>e$ vor der Kontraktion, denn der Wandel in der 2. Silbe dreisilbiger Wörter ${ }^{*} a>e$ hat kaum früher als in der 3. Silbe eintreten können. Die Entwicklung ${ }^{*} a>e$ lässt sich auch nicht erst nach der Kontraktion annehmen (also ${ }^{*} a+{ }^{*} a>a>e$ ), denn das bei der Kontraktion ${ }^{*} a+{ }^{*} \check{e}$ entstandene $a$ hat sich erhalten. Wir rekonstruieren also lieber ${ }^{*} e+{ }^{*} e$ als ${ }^{*} e+{ }^{*} a$ oder ${ }^{*} a+{ }^{*} a$. 
U Malä (Schlachter) Kontr.-*n: 2. Du.Imp. gà̀rròn 'nähen' (<*kninnen); Kontr. -*u: 1. Du.Pers.-Pron.Gen. mūnan, Gen.Sg. buutsan 'Rentier' (<*pnc̀ŭjĕn <*-njèn, Nom.Sg. bååtsòi $\left.<{ }^{*} p^{n} z^{n j}\right)$, 3. Pl.Prät. gùrran 'nähen' (<*knìüjĕn

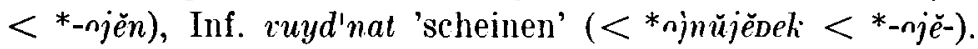
Im Dialekt von Malå ist in der 2. Silbe $i$ und $u>a$ geworden (Schlachter, Wb. des Waldlappendial. von Malå S. XIII).

Pi (Halász) Kontr. -*n: 1. Du.Imp. II ähhcun 'lieben' (< ${ }^{*}$ ec̀cojen; das $\ddot{a}$ der 1 . Silbe zeigt, dass in der 2 . Silbe $u<{ }^{*}$, vgl. 2. Sg.Imp. II ihhcu $<{ }^{*}$ ec̀cǔjĕk, wo ein Kontr.-u steht); Kontr. ${ }^{*} u$ : 1. Du. Pers.-Pron. Gen. munū (n) (Nom. mōi, mōjo), Inf. svärrūt 'antworten' $\left(<^{*}-\tilde{u} j \ddot{e}^{-}\right)$u.a. Formen der uje-Verben.

L J (Grundström) Kontr.- *n: 1. Du.Imp. II $v^{u} \dot{o} l^{p} k \bar{\omega} n$ 'fah-

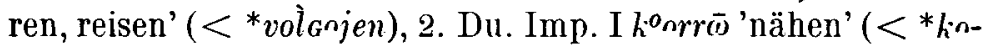
impen), Part.Prs. $k^{o}$ nirm $\bar{\omega}\left(<{ }^{*} k_{i} \dot{r} n j e\right)$; Kontr. -*u: Gen.Sg. $p^{o} n h \bar{t} s u$ 'Rentier' (s. oben), 1. Du. Pers.-Pron. Gen. munnu,

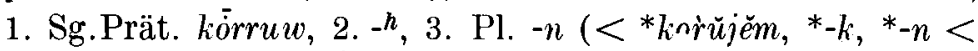
*-njèm usw.), Inf. tolòkut 'dolmetschen' u.a. Formen der ujeVerben.

N (Nielsen) Kontr. -*n: 1. Du.Imp. gul'lo 'hören' $\left(<{ }^{*} k \grave{\text { ŭ }}\right.$ lnjen), 2. Du.Imp., Part.Prs. goar'ro s. oben; Kontr. -*u: Gen., Akk.Sg. sul'lu 'Insel' $\left(<{ }^{*}\right.$ solüjën $<{ }^{*}$-njën, Nom.Sg. suolo $<{ }^{*}$ solnj), 1. Du. Pers.-Pron. Gen. mon'nu, mun'nu s. oben, 1. Sg.Prät; gōrrum, 2, -uk, 3. Pl. $-u$ s. oben, dug'gjut 'Handarbeiten machen' u.a. Formen der uje-Verben.

I (E. Itkonen) Kontr. -*n: 1. Du.Imp. kullōn s. oben, 1. Du. Prs. kott'š̄on 'nennen, rufen' (<*koččcojen), 2. Du.Imp. kottšō

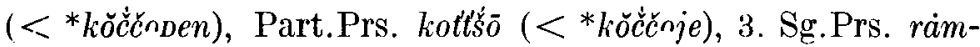
mō 'prahlen, rühmen, preisen' $\left(<*^{*}\right.$ raìnja $)$, 3. Pl.Prs. or̀̀̀h 'sein' (<*ŏŕnjeh); Kontr. -*u: Gen.,Akk.Sg. sǔŏllū 'Insel' s. oben, 1. Du.Pers.-Pron. Gen. munnū s. oben, 1. Sg.Prät.

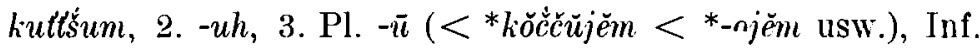
rammud u.a. Formen der uje-Verben.

Sk. Snk. Kontr. -*n: 3. Pl.Prs. särn̆na 'sprechen' $\left(<{ }^{*}\right.$ sarnnjek); Kontr. -*u: Gen., Akk.Sg., Nom.Pl. sǔŏ̄̃o (E.I.)

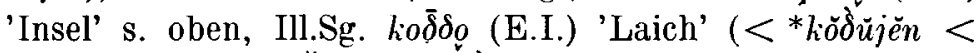

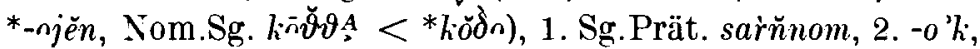




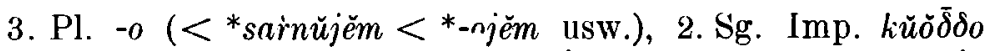
'bleiben, verlassen werden' $\left(<{ }^{*} k o \delta\right.$ ŭjĕk $<{ }^{*}-n j e ̆ k$, Inf. kŭy̆d̆$\delta i j e D)$.

Für Kld. Kontr. - $*_{n}$ kein Beleg, vgl. nichtkontr. ${ }^{*}$ : Trb.

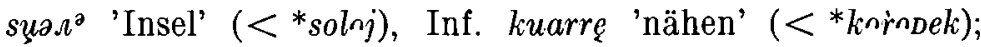
Kontr. -*u: Gen., Akk.Sg., Nom.Pl. sùлse s. oben, Ill.Sg. So pā $л л \varepsilon$ (E.I.) 'Ball' (< ${ }^{*}$ pallŭjĕn < * *njĕn, Nom.Sg. pāas),

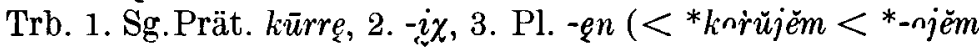
usw.). Das $*_{n}$ und $*_{u}$ der 2 . Silbe hat sich nicht getrennt erhalten, doch lässt sich der Unterschied an der Vokalquantität der 1. Silbe darin sehen, dass in den Formen mit einem urspr. ${ }^{*} u$ in der 2 . Silbe in der ersten Silbe ein engerer Vokal steht als in den Formen mit einem urspr: $*_{n}$ in der 2. Silbe.

$\mathrm{T}$ (T. I. Itkonen) für Kontr.- ${ }^{*}$ kein Beleg, vgl. nichtkontr. $*_{n}$ : siầıai 'Insel', Inf. kóarra ${ }^{d}$ 'nähen'; Kontr. -*u: Gen., Akk.

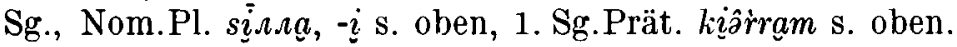

Über die labialen Kontraktionsvokale und den dialektal in einigen Formgruppen des Westlappischen vollzogenen Wandel $*_{n}>{ }^{*} u$ s. E. Itkonen MSFOu 79 S. 303-304, 354-373.

Da der Kontraktionsvokal bei den Kontraktionen ${ }^{*} a+{ }^{*} \check{e}$ $>{ }^{*} a,\left({ }^{*} a+{ }^{*} a>\right){ }^{*} e+{ }^{*} e>{ }^{*} e$ und $\left({ }^{*} n+{ }^{*} a>\right){ }^{*} n+{ }^{*} e$ $>*_{n}$ die Qualität des ersten der betroffenen Vokale befolgt, müsste auch das Kontraktions- ${ }^{*} u$ qualitätsmässig dem ersten jener Vokale entsprechen, aus deren Kontraktion es entstanden ist, also Kontr.- ${ }^{*} u<{ }^{*} \breve{u}+{ }^{*} \check{e}\left(<{ }^{*} n+{ }^{*} \breve{e}\right)$. Auch die Kontraktion $*_{\breve{e}}+*_{\breve{e}}>i$ richtet sich nach demselben Grundsatz, denn das $*_{\breve{e}}$ war im Urlp. offenbar eng (s. a.a.O. 343).

Das Kolalappische aber scheint auch auf einen Unterschied zwischen nichtkontrahiertem $*_{n}$ und $*_{u} u$ hinzuweisen. Sowohl bei Arvid Genetz als auch bei T. I. Itkonen finden wir Formen, wo vor einem engen Vokal der 3. Silbe in der 2. Silbe der Vertreter eines * $u$ und nicht eines $*^{*}$ steht. Zwar ist die Vertretung dieser Vokale oft zusammengefallen, doch kann die urspr. Vokalqualität der 2. Silbe aus der Vokalvertretung in der 1. Silbe geschlossen werden, denn durch den Einfluss der Metaphonie sind die meisten urlp. Vokale im Kolalappischen vor einem $* u$ der 2 . Silbe enger vertreten als vor einem $*_{n}$ (s. E.Itkonen, a.a.0. 8-43). Die Aufzeichnungen von Genetz und 
T. I. Itkonen geben kein einheitliches Bild vom Labialvokal der zweiten Silbe vor einem engen Vokal der dritten Silbe. Einige Fälle weisen auf ein ${ }^{*} u$ hin, andere auf ein ${ }^{*}$, z.B. ${ }^{*} u$ :

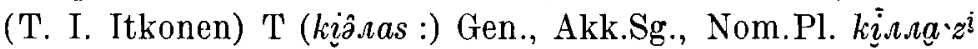
'Reihe unangespannter Rentiere, deren Riemen (Zügel) jeweils am Hals des vorhergehenden Tieres festgebunden ist'

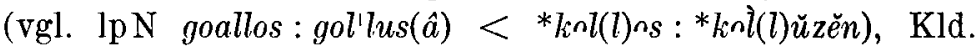

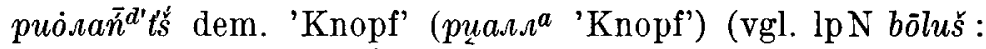
boallo $<{ }^{*}$ pnlüñ̌žĕ: *pnl̀n), T. (viâzvan :) Gen., Akk.Sg., Nom. Pl. vis̄va 'n $n^{i},-i \cdot n^{i}$ 'Barsch' (vgl. lp N vuosko : vus'kun(â)), Kld. rūps ${ }^{v}$ ne $e^{\delta}$ 'rot werden, erröten' (vgl. lpN rüvsudit < ${ }^{*}$ ropsŭnDë-), T pĩ dai dem. 'Damm' (pî̀nta 'Damm') (vgl. lp N büduš :

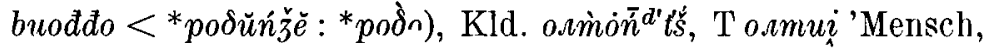
Mann' (vgl. Ip N olmušs), Kld. kiinnañ ${ }^{d^{\prime}} t^{\prime \prime s}$ dem. 'Richtung od. Platz des Winterwegs' (kéăân ${ }^{(a)}$ 'Richtung od. Platz des Winterwegs') (vgl. Ip N gēinuš́ : gei'dno), Kld. pūrrgbñ ${ }^{d} t^{\prime} t^{\prime}$ dem. 'Bremse' (puara 'Bremse') (<* *príŭjēêň̌̌ : *pnrnj); (Genetz) T villkine-, -lginta 'gebleicht werden' (vgl. $1 \mathrm{pN}$ vilgudit $<$ *velaŭnDë-), Kld. olmuńč́, T olmij 'Mensch' usw.; *n: (T. I. Itkonen) Kld. (sëam ${ }^{a}$ :) Gen., Akk.Sg., Nom.Pl. seäamman, T

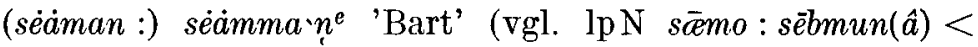
*seman : *scmŭunĕn), KId. (sàv ${ }^{a}$ :) sàvvan 'stillstehendes Wasser zwischen zwei Stromschnellen' (vgl. IpN sâvo : sâvvun $(\hat{a})<$

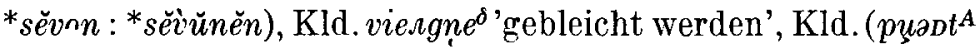

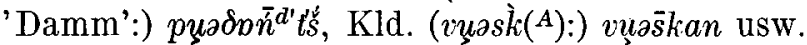

Erkki Itkonen hat in seiner Untersuchung der Vokale auf den terlappischen Verbtypus ćärkinta 'verbleichen, vergilben (von Pelzwaren)', vilginta 'weiss werden' hingewiesen, wo scheinbar eine Fortsetzung des $* \check{u}$ der 2 . Silbe steht, doch ist es s.E. nicht wahrscheinlich, dass es sich um eine Entsprechung des westlp. ${ }^{*} \breve{u}$ handelt (MSFOu 79 S. $167-168$ ). Wie die oben stehenden Beispiele zeigen, gibt es in zahlreichen kildinund terlp. Ableitungstypen Anzeichen für einen Wandel $*_{n}$ $>*_{u}$, aber nicht regelmässig: oft scheint der Wechsel $*_{n} \sim$ $*_{u} \breve{u}$ zugunsten von $*_{n}$ ausgeglichen worden zu sein.

Es wirkt unwahrscheinlich, dass der Wandel $*_{n}>*_{u}$ im Kolalappischen unabhängig von dem entsprechenden westlappischen Lautwandel eingetreten wäre, denn auch die Qua- 
lität der labialisierten Kontraktionsvokale ${ }^{*} u\left(<{ }^{*} \check{u}+{ }^{*} \check{e}\right)$ und $*_{n}\left(<*_{n}+*_{e}\right)$ im Inari- und Skoltlappischen weist hin auf einen urlp. Wandel $*_{n}>*_{\breve{u}}$. In diesern Zusammenhang braucht nicht überlegt zu werden, weshalb das $*_{n}$ im Inari- und Skoltlp. vollständig und im Kolalp. teilweise wieder eingeführt wurde. Beachtung verdienen jedoch zwei inarilp. Wörter, wo entgegen unserer Erwartung in der 2. Silbe

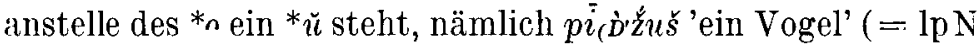

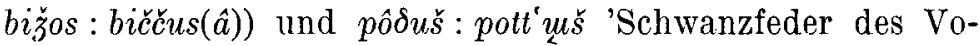
gels' (= $\operatorname{lp~N}$ bâdoš : bâttuš(â)) (s.E. Itkonen a.a.O. 112). Vielleicht wurde der Wechsel $*_{n} \sim *_{\breve{u}}$ in diesen Wörtern ausnahmsweise zugunsten von $* \breve{u}$ ausgeglichen.

Wenn das $* \breve{u}$ in der zweiten Silhe vor den urlappischen Kontraktionen existierte, war es auf jeden Fall ein Allophon von ${ }^{*} n$. Das Verhältnis zwischen ${ }^{*} a$ und ${ }^{*} e$ ist ein anderes, denn ${ }^{*} a$ hatte sich ausnahmsweise auch in der letzten Silbe von Formen der singularischen Personen der $e$-stämmigen Verben erhalten (s. MSFOu 143 S. 190 und das dort angegebene Schrifttum). Es lassen sich Minimalpaare rekonstruieren wie 2. Sg.Prs. *pnoak-- 2. Sg.Imp. *pnoek (>1pN boadak boadě) 'kommen'. Die funktionale Belastung der Opposition ${ }^{*} a-{ }^{*} e$ war jedoch ausserordentlich gering, denn hauptsächlich diese Vokale standen in komplementärer Distribution. Auch das im Urlp. vor ${ }^{*} j$ und einem mouillierten Konsonanten aus ${ }^{*} e$ entwickelte $i$ war ein Allophon des ${ }^{*} e$ (z.B. $\operatorname{lpN} 3$. Sg.Prät. böđii $<{ }^{*} p^{n}$ D $\ddot{j}<{ }^{*} p^{n}$ Dej $<{ }^{*} p^{n}$ Daj). Die Anzahl der Vokalphoneme stieg also im Urlp. wegen dieser Veränderungen nur um eins, da $*_{a}$ sich aufteilte in $*_{a}$ und $*_{e}$.

Die von Erkki Itkonen rekonstruierten urostlp. Kontraktionsvokale ${ }^{*} a,{ }^{*} e,{ }^{*} i,{ }^{*}$ (bei Itkonen ${ }^{*} \omega$ ) und ${ }^{*} u$ (Itkonen a.a.O. 310) begegnen alle auch im ganzen Westlappischen, und sogar hauptsächlich in denselben Formgruppen wie im Ostlappischen, so dass sie als urlappisch gelten können (vgl. S. 246). Die Entstehung von Kontraktionsvokalen bedeutete eine beachtliche Bereicherung des Vokalsystems der zweiten (und vierten) Silbe im Urlp. Anfangs waren offenbar alle Kontraktionsvokale selbständige Phoneme, die sich von den nichtkontrahierten Vokalen zumindest quantitätsmässig unterschieden, denn vor ihnen wurde der starkstufige Vertreter eines Einzel- 
konsonanten unter bestimmten Voraussetzungen geminiert (zur Überdehnung s. 236-237). Später verschmolzen die Kontraktionsvokale mit ihren nichtkontrahierten qualitativen Entsprechungen im Westlappischen völlig und im Ostlappischen teilweise. Trotzdem hatte die Kontraktion eine beachtliche Wirkung auf das Vokalsystem der gleichen Silbe, denn sie kommt wenigstens in der Phonemisierung von $i$ und $u$ und einer vermehrten funktionalen Belastung des $a$ zum Ausdruck. Der reichere und kombinationsfähigere Vokalismus übernahm einen wesentlichen Teil der Funktionen der in der Kontraktion verlorengegangenen Suffixe. So erhielten z.B. die Kontraktionsvokale $i$ und $u$ in der 1. und 2. Sg. und der 3. Pl. die Funktion des Präteritumszeichens $j$ ( $1 \mathrm{p} \mathrm{N}$ mânnim <

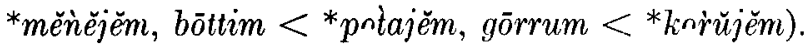

Auffallend im Südlappischen ist, dass in einigen zentralen Formgruppen einfache Kontraktionsformen ersetzt werden durch längere Formen, während die anderen Dialekte ausschliesslich den Kontraktionstypus verwenden. Dies kann zusammenhängen mit dem fehlenden Stufenwechsel. Wie oben festgestellt wurde, haben neben den Kontraktionsvokalen auch der Stufenwechsel und die Überdehnung einen Teil der morphologischen Funktion erhalten, die vor der Kontraktion die Suffixe hatten. In den meisten Fällen lässt sich die fehlende Kontraktion in südlappischen Formen leicht als Ergebnis einer späten Entwicklung feststellen, wenn sie nämlich in nördlicheren Entsprechungen vorhanden ist. Spät entstanden sind Formen wie 3. Pl. Prs. boåtiejievies 'kommen', pruwk<ajievies 'pflegen', neben denen noch der ursprüngliche Typ boåtieh, prüwkieh begegnet. Aus neuerer Zeit stammt ferner der Pleonasmus des Präteritumszeichens (boåtiejijjom, juwk<astâllajiijom), neben denen der ursprüngliche Typ fast vollständig geschwunden ist, so dass er nur noch selten, und zwar im Präteritum der $e$-stämmigen Verben, auftritt $\left(b<\ddot{o} t_{z}^{\prime} m\right)$ (s. Bergsland, Røros-lappisk grammatikk S. 159, 161). Die längere Form der 2. Du.Prs. und Imp. (urspr. wahrscheinlich nur Imp.), die auf der 2. Pl. beruht (boåtáran usw., vgl. 2. Pl. boålara, s. MSFOu 143 S. 280-281), hat die ursprüngliche Kontraktionsform abgelöst; veraltet und selten begegnet sie noch in der 2. Du.Imp. der $e$-stämmigen Verben $\left(b o a^{-}\right.$ 
tien, boåtan, s. Bergsland a.a.O.). Möglicherweise wurden diese Formen, da die urspr. Kontraktionsformen mit dem Schwund des Stufenwechsels einen wichtigen und für sie charakteristischen Zug verloren, die starke Stufe nämlich vor einer geschlossenen Silbe und eventuell die Überdehnung, ersetzt durch solche mit einem Suffix, wodurch ihre Funktionen deutlicher ausgedrückt waren. Wenn das zuträfe, hätten wir einen neuen interessanten Aspekt im Verhältnis zwischen Wechseln im Stamminnern und morphologischer Methode. Wir haben uns bisher mit Fällen beschäftigt, wo mit der Entwicklung von Wechseln im Stamminnern ein Verfall der Suffixe verbunden war. Die hier nun behandelte südlappische Erscheinung wäre ein Beispiel für das Gegenteil: der Ausgleich eines Wechsels inı Innern des Stammes, in diesem Fall des Stufenwechsels, hätte dazı geführt, dass fusionierend-symbolische Formen ersetzt wurden durch fusionierend-agglutinierende Formen. Mit dem oben Dargelegten harmoniert die Tatsache, dass die für das Südlappische typischen pleonastischen Formen bereits im Umelappischen fehlen, wo es Stufenwechsel gibt, das aber sonst eng mit dem Südlappischen verbunden ist.

Der Anteil der Metaphonie an den morphologisch distinktiven Wechseln im Innern des Stammes ist erstaunlich gering. Mit Ausnahme einiger Fälle, wo die Metaphonie der zweiten Silbe distinktiv ist (z.B. die weit verbreitete Opposition zwischen Part.Perf. und Nomen act. der $e$ - und $o$-stämmigen Verben: lp $\mathrm{N}$ giel'dam - giel'dem usw.), beschränkt sich die morphologische Distinktivität der Metaphonie auf das Süd- und Ostlappische. Das ist natürlich, denn im Pite-, Lule- und Norwegischlappischen begegnet wenig Metaphonie der betonten Silbe. Doch auch im Süd- und Ostlappischen ist die Metaphonie der betonten Silbe nur in einigen Oppositionen distinktiv, obgleich sich die Metaphonievarianten in grossem Ausmass zu selbständigen Phonemen entwickelt haben. Zur Erläuterung dieser Angelegenheit soll untersucht werden, unter welchen Voraussetzungen die Metaphonie morphologisch distinktiv wurde. Die Grundbedingung ist, dass vor der Metaphonie der sie auslösende Vokal paradigmatisch wechselt mit einem keine Metaphonie bewirkenden Vokal oder einem Vokal von anderer metaphonischer Wirkung und dass sich 
dieser Wechsel nach der Metaphonie ausgleicht. Zweitens ist vorauszusetzen, dass die früheren Morphe schwinden oder ihre Distinktivität in dem Masse verlieren, dass die als Ergebnis der Metaphonie entstandenen Vokaloppositionen die einzig morphologisch distinktiven Faktoren bleiben.

Sind diese Voraussetzungen nun gegeben in den lappischen Metaphoniedialekten, dem Süd- und Ostlappischen? Die oben besprochenen (S. 284, 288), unter bestimmten Bedingungen für den Vokal der zweiten Silbe geltenden qualitativen Veränderungen ${ }^{*} a>{ }^{*} e,{ }^{*} e>{ }^{*} \breve{\imath}$ und ${ }^{*} n>{ }^{*} \breve{u}$ führten in den $e\left(<{ }^{*} a\right)$ und $o$-stämmigen Wörtern zu paradigmatischen Vokalwechseln in der zweiten Silbe (vgl. lp N jâkke Ill.Sg. jâkkai $\sim$ Kom.Sg., Lok.Pl. jâgiin 'Jahr', dâkko Ill.Sg. dâklkui 'Tat'). Die Kontraktionsvokale haben die Vokalwechsel so vielseitig gemacht, dass in den meisten Flexionstypen von lappischen gleichsilbigen Nomina und Verba in der zweiten (oder vierten) Silbe ein paradigmatischer qualitativer Vokalwechsel auftritt oder auftrat. Ausserhalb dieser alten Wechsel stehen in allen Mundarten nur die relativ jungen und seltenen Nomina mit einem $a$-, $\check{\imath}$ - und $\check{u}$-Stamm. Im Südlappischen begegnet auch bei $\breve{a}$-stämmigen Substantiven kein paradigmatischer Wechsel des Vokals der zweiten Silbe.

Die den Vokalwechseln der zweiten Silbe entsprechenden Metaphoniewechsel im südlappischen Dialekt von Röros sehen folgendermassen aus (die Angaben gründen sich auf Knut Bergslands Darstellung, s. Røros-lappisk grammatikk S. 82-85):

Tabelle 6.

\begin{tabular}{|c|c|c|c|c|c|c|c|c|}
\hline \multirow{3}{*}{$\begin{array}{l}\text { in der } \\
\text { 2. Silbe }\end{array}$} & Stammtypus & \multicolumn{3}{|c|}{$e$} & \multicolumn{2}{|c|}{$o$} & \multicolumn{2}{|c|}{$\breve{a}$} \\
\hline & Urlp. & $*^{*} a$ & ${ }^{*} e$ & $*_{i}$ & $*_{n}$ & ${ }^{*} u$ & $* \check{e}$ & ${ }^{*} a$ \\
\hline & Rör. & $a \partial$ & ie a & a & $a \partial$ & $\partial$ & $a$ & $a$ \\
\hline & in der 1. Silbe & $\hat{a}$ & $\ddot{a}$ & $i$ & $\dot{a}$ & $u$ & $\hat{a}$ & $\hat{a}$ \\
\hline & & $\ddot{a}$ & $i$ & $i$ & $\ddot{a}$ & $\ddot{u}$ & $i(\dot{u})$ & $\ddot{a}$ \\
\hline & & $\hat{a}$ & $\dot{u}$ & $\dot{u}$ & $\dot{a}$ & $u$ & $u$ & $\hat{a}$ \\
\hline & & $a$ & $a$ & $e$ & $a$ & $o$ & $a$ & $a$ \\
\hline & & $e \mathscr{X}$ & $i e$ & $e$ & $e \mathscr{X}$ & $\ddot{o} 0$ & $\boldsymbol{e} \boldsymbol{X}$ & $e \mathscr{X}$ \\
\hline & & $e \mathscr{E}$ & ie & $e$ & $e \not{e}$ & $\ddot{o} 0$ & $i e$ & $e \mathscr{e}$ \\
\hline & & $o a ̊$ & $o \dot{a}$ & $b e$ & $o \mathfrak{a}$ & $o$ & $o \dot{a}$ & $o \dot{a}$ \\
\hline & & $u \mathscr{X}$ & uo & $\ddot{o} e$ & $\boldsymbol{o} \dot{a}$ & $o$ & uo & $u \circledast$ \\
\hline
\end{tabular}


In der Flexion der Substantive begegnet nur der Wechsel ${ }^{*} a \sim{ }^{*} e$ in der zweiten Silbe und die metaphonisch darauf beruhenden Wechsel im Vokalismus der ersten Silbe. Im Dual der Personalpronomina und in einigen Partikelparadigmen tritt in der 2. Silbe auch der Wechsel $*_{n} \sim{ }^{*} u$ auf und die davon abhängigen metaphonischen Wechsel in der 1 . Silbe. In der Konjugation haben wir in der 2. (resp. 4.) Silbe die Wechsel ${ }^{*} a \sim{ }^{*} e \sim{ }^{*} i\left({ }^{*} i\right.$ selten im Prät.), ${ }^{*} \sim^{*} u$ und ${ }^{*} \check{e} \sim{ }^{*} a$ und die ihnen entsprechenden Wechsel im Vokalismus der 1. Silbe. S. Bergsland a.a.0. 100--120, 161-171. Paradigmatischer Metaphoniewechsel erscheint nicht in jenen e-Stämmen, in deren erster Silbe ein Vertreter des ${ }^{*} a$ oder $*_{n}$ steht, denn als Vertreter von $*^{*} a$ und $*_{n}$ steht sowohl vor $*^{*} a$ als auch

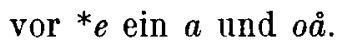

Eine Neutralisierung der Vokaloppositionen in der 2. Silbe -- die unumgängliche Voraussetzung für eine Distinktivität der Metaphonie - ist im Südlappischen häufig eingetreten, die zur Behandlung stehenden paradigmatisch wechselnden Vokale aber werden davon nur im dreisilbigen Takttypus betroffen (Bergsland: "Takttypus B", vgl. z.B. "Takttypus A" goaitie : El.Sg. "Takttypus B» goåtasto 'Zelt, Hütte', s. a.a.O. 80), wo alle urlp. Vokale durch a vertreten sind. Der Unterschied zwischen vorderen und hinteren Vokalen hat sich jedoch darin erhalten, dass der Inlautkonsonantismus vor einem velare Vokale vertretenden z velar ist, im anderen Falle palatal (a.a.O. 82), vgl. z.B. 2.Pl.Imp. I allaŕa $<*^{*}$ ale-, Imp.II ultaŕ $<* a \grave{a l u ́-~}$ (Verneinungsverb). Nach den phonetischen Bezeichnungen von Lagercrantz (Lappischer Wortschatz, Helsinki 1939) und Collinder (Lappische Sprachproben aus Härjedalen, Uppsala 1942) hat a ebenfalls ein vorderes und hinteres Allophon.

Auf die morphologische Verwendung des Wechsels im Innern des Stammes ist die Verteilung der am Wechsel beteiligten Phoneme im Paradigma von entscheidendem Finfluss. Auch in dieser Hinsicht ist die Situation im Südlappischen nicht günstig für eine morphologische Distinktivität der Metaphonie, denn die Aufteilung der wechselnden Phoneme ist in allen Fällen sehr ungleich. So wechseln $u$ und $\hat{a}$ im Dualparadigma der Personalpronomina so, dass letzteres nur im 
Nominativ steht, ersteres in allen anderen Kasus (Nom. mânnah, Gen. munnan, Akk. munnam, Ill. munnasa usw.). In der Flexion der $e$-stämmigen Nomina begegnet der durch das $a$ der 2. Silbe vorausgesetzte Vokal der 1. Silbe nur im Ill.S von Typ goåtan, in der Verbalflexion wiederum nur in der 1 ., 2. und 3. Sg.Prs. sowie im Part.Perf., während die übrigen Formen ein $*_{e}$ in der 2. Silbe voraussetzen (im Prät. der Verben alternativ ein $* i$ ). In der 2 . (oder 4 .) Silbe der $a$-stämmigen Verben hat der Vertreter des $\check{a}$ ïberall ausser in der 3. Sg.Prs. mit einem $a$ Alleinvertretung. Im Paradigma der 0 -stämmigen Verben steht nur in der 3. Sg.Prs. und im Part.Perf. in der 2. Silbe ein Vertreter des ${ }^{*} u$ und in der 1 . Silbe die entsprechende Metaphonievariante; in der 2. Silbe aller anderen Formen begegnet ein Vertreter des $*_{n}$. Die ungleichmässige Verteilung der Wechselvokale ist in einigen Fällen das Ergebnis einer späten Entwicklung. Z.B. sind im Präteritum der $o$-stämmigen Verben die Formen mit einem $* u$ in der 2 . Silbe (vgl. lp N 1. Sg.Prät. gōrrum, 2. gōrrul, 3. Pl. gōrru) von den pleonastischen Formen mit einem $*_{n}$ gänzlich verdrängt worden (Rör. dajtajijjam usw.). Entsprechend gibt es im Stamm der $\breve{a}$-stämmigen Verben keinen Metaphoniewechsel, bewirkt durch einen Wechsel $\breve{a} \sim i$ in der 2 . (oder 4.) Silbe, da pleonastische Präteritumsformen den Platz der alten Formen mit $i$ einnahmen (Rör. juwk<astâttajijjam usw., vgl. $\operatorname{lp~N}$ mânnim usw.). Wenn beim paradigmatischen Metaphoniewechsel einer der Wechselvokale in fast allen Formen des Paradigmas erscheint und der oder die anderen nur in einer oder zwei Formen, können Situationen, wo der Wechsel morphologisch distinktiv würde, natürlich nicht so leicht entstehen wie dann, wenn jeder Wechselvokal in ungefähr gleich vielen Formen des Paradigmas erscheint.

Die zweite Hauptvoraussetzung der morphologischen Distinktivität der Metaphonie, der Schwund oder die Schwächung der Distinktivität der ursprünglichen Morphe, hat sich im Südlappischen nur in geringem Ausmass verwirklicht. Einen Schwund der Schlusslaute des Wortes gibt es im Südlappischen kaum und auch Kontraktionsfälle sind seltener als in den nördlicheren Mundarten. Dagegen begegnet ein mit der 
Reduzierung des Vokalismus der nichtersten Silben verbundenes Erlöschen der Distinktivität der suffixalen Vokale. Im zweisilbigen Takttypus ("Takttypus A") haben sich das $*_{a}$, ${ }^{*} i\left({ }^{*} \check{e}=\right.$ gekürztes etym. langes $\left.e\right)$ und $*_{u}\left({ }^{*} \check{o}=\right.$ gekürztes etym. langes $*_{n}$ ) zu a entwickelt und im dreisilbigen Takttypus ("Takttypus B") sind alle Vokale der 2. Silbe und von denen der 3 . Silbe $*_{\breve{a}}$ und $*_{e}$ als $a$ vertreten. Wie oben festgestellt wurde, hat sich die Korrelation der vorderen und hinteren Vokale im vorangehenden Konsonantismus teilweise erhalten. Die Neutralisierung der Vokaloppositionen in den nichtersten Silben hat jedoch nur sehr wenig beigetragen zu einer Vereinheitlichung der Flexionsendungen, denn die überwiegend gebräuchlichen Suffixvokale waren ${ }^{*} \check{a}$ und ${ }^{*} e$, ein hinterer und ein vorderer Vokal. Der Einfluss macht sich eher im Grenzvokalismus geltend, der früher bedeutend reicher war als heute. Durch die Neutralisierung der Oppositionen des Grenzvokalismus wurde der Metaphoniewechsel zwar phonologisch distinktiv, ein morphologisch distinktiver Wechsel aber wurde dadurch kaum bewirkt.

In den ostlp. Dialekten erscheinen die paradigmatischen Vokalwechsel der gleichen Silbe des Urlp. entweder als solche oder - metaphonisch übertragen auf die vorangehende Silbe - besser als im Südlappischen. Nur der eventuelle Wechsel zwischen nichtkontrahiertem $*_{n}$ und $*_{\breve{u}}$ hat sich im Skolt- und Inarilappischen und zumindest teil- und dialektweise im $\mathrm{Ko}_{0}$ lalappischen ausgeglichen. Dieser Ausgleich wurde zugunsten des $*_{n}$ vollzogen (s. S. 286-288). Der ostlappische Vokalismus und vor allem auch die Metaphonie sind ausführlich behandelt worden von Erkki Itkonen (MSFOu 79 besonders S. 1-43). Auf den Seiten 41-43 seiner Arbeit gibt er in Form von Tabellen einen Überblick über die Abhängigkeit der ostlp. Vokalqualität der 1. Silbe von der der 2. Silbe. Anhand dieser Tabellen kann man sich leicht ein Bild machen von den paradigmatischen Metaphoniewechseln, die den Vokalwechseln der 2. Silbe entsprechen. In diesem Zusammenhang brauchen die Regeln des Wechsels nicht noch einmal wiederholt zu werden. Es genügt die Feststellung, dass der paradigmatische Metaphoniewechsel beträchtlich häufiger ist als im Südlappischen. 
Im Inarilappischen hat sich die Metaphonie schwächer entwickelt als im Skoltlappischen (vgl. S. 241). Bei den Vertretern von urlp. ${ }^{*} i$ und ${ }^{*} \check{u}$ ist sie gar nicht feststellbar, oder sie ist sehr gering. Der Metaphoniewechsel der Vertreter von urlp. $*_{\check{e}}$ und $*_{\check{o}}$ ist zweistufig, der der übrigen Vokale dreistufig. Im Skoltlappischen ist der Metaphoniewechsel 3-4stufig, im Kildinlappischen kann man wohl 2-4 Stufen und im Terlappischen 1-.3 Stufen unterscheiden.

Ein Schwund der Vokaloppositionen in der zweiten Silbe begegnet im Ostlappischen nicht in dem Masse wie im Südlappischen. Wenn man die Verschmelzung des urlp. nichtkontrahierten ${ }^{*} \breve{u}$ mit ${ }^{*}$ ausser acht lässt, hat das Inarilappische in den Hauptzügen alle urlp. Vokale der 2. Silbe qualitativ voneinander getrennt erhalten. Unter bestimmten Bedingungen ist jedoch eine Überschneidung zu beobachten. Die Vertretung von urlp. ${ }^{*} e$ und $*_{n}$ ist qualitativ und quantitativ unter bestimmten Voraussetzungen nach einem etym. langen Vokal der 1 . Silbe mit der von $* i$ und * $u$ zusammengefallen (s. E. Itkonen a.a.O. 132-133, 158-160). Gerade dieser Umstand verursachte den einzigen mir begegneten Fall im Inarilappischen, wo die Metaphonie der 1. Silbe allein morphologisch distinktiv ist (s. S. 265).

Im Russischlappischen ist das $*_{n}$ der 2 . Silbe völlig mit $*_{a}$ zusammengefallen (a.a.O. 166). Die Opposition von $*_{a}$ und $*_{n}$ hat sich nicht einmal metaphonisch auf die erste Silbe übertragen und erhalten, denn der Einfluss beider Vokale auf die vorangehende Silbe war derselbe. Für die Metaphonie ist es auch nicht von Bedeutung, dass zumindest im Dialekt von Paatsjoki das Kontraktions- ${ }^{*} u$ der 2 . Silbe ebenso vertreten ist wie das ${ }^{2}$, nämlich als $\hat{\boldsymbol{n}}$, da beide dieselbe Wirkung auf den Vokal der 1. Silbe ausübten (z.B. Part.Perf. kīttŷm<

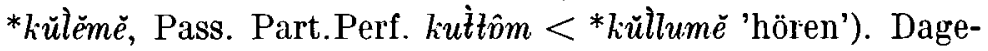
gen hat der im Skoltlappischen und wenigstens teilweise auch im Kolalappischen eingetretene Zusammenfall von $*_{i}$ und $*_{e}$ der 2. Silbe (a.a.0. 101-, 133-, 322-, 337-) den Übergang der Opposition auf die 1. Silbe veranlasst. Im Skoltlappischen ist die metaphonische Wirkung des * $i$ auf den Vokal der 1. Silbe generell eine andere als die von ${ }^{*} e$. Und doch ist die Ver- 
tretung eines $* \breve{u}$ der 1 . Silbe vor eineni nichtkontrahierten $*_{e}$ dieselbe wie vor $*_{i}$. In Nuortijärvi (Notozero) kann auch in der Vertretung von ${ }^{*} \varepsilon$ und ${ }^{*} e$ eine gewisse Neutralisierung sichtbar werden, obgleich die zur Verfügung stehenden Quellen kein eindeutiges Bild geben. In gewissem Ausmass unklar bleibt auch der Stand im Kolalappischen. Nach E. Itkonen sind im Kildinlappischen zumindest $*_{\check{e}},{ }^{*} a,{ }^{*} \varepsilon$, ${ }^{*}$ n und $*_{0}$ vor $* i$ anders vertreten als vor ${ }^{*} e$, während es wiederum im Terlappischen kaum Unterschiede gibt (a.a.0. 41-43).

In den meisten russischlappischen Dialekten lässt sich eine Überschneidung der Vertretung von $*_{\check{e}}$ und $*^{*} a\left(*_{n}\right)$ in der 2. (und 4.) Silbe feststellen. Im skoltlappischen Dialekt von Paatsjoki sind ${ }^{*} a$ und $*_{n}$ unter bestimmten Bedingungen durch $\hat{p}$ vertreten, das gleichzeitig die normale Fortsetzung von $*_{\check{e}}$ darstellt (a.a.O. 119-120). In Suonikylä ist das nicht so. Die dort vorhandenen begrenzten Neutralisierungen $\left({ }^{*} \check{e}\right.$, $*_{a}>a, \underset{>}{i}$, a.a.O. 84, 120-121) sind für unser Thema bedeutungslos. In Nuortijärvi und im Kolalappischen scheinen $*_{\ddot{e}}$ und ${ }^{*} a$ wiederum ziemlich allgemein zusammengefallen $\mathrm{zu}$ sein, wenn es auch schwer ist, ein genaues Bild über ihre Vertretung zu erhalten (a.a.O. 84-85, 121). Im Skoltlappischen war der Einfluss des $* \breve{e}$ der 2 . Silbe auf alle Vokale der 1. Silbe ein anderer als der von $*_{a}$ und $*_{n}$. Auch im Kolalappischen besteht in der Vertretung der meisten urlp. Vokale der 1. Silbe ein Unterschied, je nachdem, ob in der 2 . Silbe ein * ${ }_{\check{e}}$ oder ein ${ }^{*} a\left(*_{n}\right)$ gestanden hat (s. a.a.O. 41-43).

Die behandelten Neutralisierungen der Vokaloppositionen der 2. Silbe im Russischlappischen waren weitgehend von Einfluss darauf, dass der Metaphoniewechsel phonologisch distinktiv wurde. Für die morphologische Methode dagegen war - wie wir feststellten - nur das Zusammenfallen von ${ }^{*} e$ und $*_{i}$ der gleichen Silbe im Dialekt von Suonikylä von Bedeutung (s. S. 271, 273). Ein paradigmatischer Wechsel ${ }^{*} \boldsymbol{\sim} *_{i}$ begegnet ja bei den $e$-stämmigen Nomina und Verben. Einen Wechsel $*^{*} \sim *_{a}$ haben nur die $\breve{a}$-stämmigen Verben, und auch dort steht die Fortsetzung von $* a$ nur in der 3 . Sg. Obgleich sich also im Russischlappischen ein durch Metaphonie verursachter, häufiger paradigmatischer Vokalwechsel in der 
betonten Silbe entwickelt hat, ist er zumindest im Dialekt von Suonikylä meist morphologisch redundant geblieben. Aufgrund der obigen Ausführungen kann als Hauptursache dafür bezeichnet werden, dass die Vokaloppositionen in der nachfolgenden Silbe verhältnismässig gut erhalten sind.

Die zweite Grundvoraussetzung für die morphologische Distinktivität des Metaphoniewechsels, dass die früheren Morphe schwinden oder ihre Distinktivität verlieren, ist im Russischlappischen gut verwirklicht. Nirgendwo anders im Lappischen sind die Suffixe derart verbraucht wie im Russischlappischen. Doch die Funktionen der geschwundenen Suffixe und die verlorene Distinktivität gingen grösstenteils über auf andere Wechsel im Innern des Stammes als es die metaphonischen gewesen wären (s. S. 270--274).

Die obige Darstellung beruht auf dem Dialekt von Suonikylä. Der Anteil der Metaphonie an der Morphologie der anderen russischlappischen Dialekte sieht etwas anders aus. In Paatsjoki sind durch den Zusammenfall von $*_{\check{e},}{ }^{*} u,{ }^{*} a$ und ${ }^{*}{ }_{n}$ unter bestimmten Bedingungen einige im Dialekt von Suonikylä unbekannte Fälle entstanden, wo der Metaphoniewechsel - meist neben irgendeinem quantitativen Wechsel - morphologisch distinktiv ist, z.B. (E. Itkonen) Nom.Sg. $a i \bar{r}^{b}$ Ill.Sg. oaīî̀ 'Ruder' (<*ajrn-- *ajrun <*ajrŭjĕn), 1. Du., 3. Pl.Prs., 1. Du.Imp. àn̄n̂ि-3. Pl.Prät., Imp. II neg. oañnî 'ersuchen' (<*à̀nnn, *-k-*à̀nun, ${ }^{*}-k$ ) (in Suonikylä 3. Pl.Prs. - 3. Pl.Prät. nur bei $\check{a}$ - und $e$-stämmigen Verben, vgl. $o$-stämm. Snk. ànna $-\cdots a \bar{n} n o)$, 3. Sg.Prs. $t a^{0} \bar{p} p^{A}-2$. Sg. Imp. $t \bar{\varepsilon}^{J} p p^{A}$, Imp. II neg. $t \varepsilon^{3} \bar{p} p \hat{v}$ 'verstopfen, schliessen' $(<* t e \grave{p}-$ $\left.p a-{ }^{*} t e \check{p} p e \check{k}-{ }^{*} t e \check{p} p u h\right)$. Im Kildinlappischen ist der Ausgleich der Vokaloppositionen der 2. Silbe und auch der Schwund der suffixalen Elemente noch weiter gegangen als im Skoltlappischen. Die Fälle mit morphologisch distinktiven Vokalwechseln durch Metaphonie sind nicht identisch mit denen im Skoltlappischen. Im Kildinlappischen begegnet die auf Metaphonie beruhende Opposition 3. Pl.Prs. - 3. Pl.Prät. nicht, da dort in diesen Formen die Personalendung auftritt, z.B. (E.Itkonen) 3. Pl.Prs. puaple $\beta$ - 3. Pl.Prät. püptẹn 'kommen', vgl. Snk. pŭăățte - pư̆ăōtte. Andererseits finden wir im 
Kildinlappischen Paare vor wie 1. Sg.Prät. kūrrę-2. Pl.

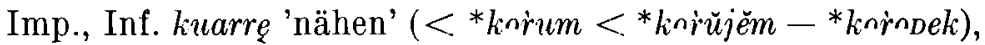
die im Skoltlappischen nicht bekannt sind. Obgleich in dieser Hinsicht über das Kildinlappische nichts Endgültiges ausgesagt werden kann, bevor der Vokalismus der nichtersten Silben aufgrund eines sorgfältig gesammelten Materials unter Berücksichtigung der Dialektunterschiede genau untersucht ist, erscheint es jedoch schon aufgrund der bisherigen Dialektforschung offenbar, dass das Kildinlappische hinsichtlich der Allgemeinheit der morphologischen Distinktivität der Metaphonie nicht wesentlich abweicht von den übrigen ostlappischen Dialekten. Aller Wahrscheinlichkeit nach sind auch dort die übrigen Wechsel im Innern des Stammes wichtiger für die Morphologie als die durch Metaphonie bewirkten. Vom Terlappischen kann man noch schwerer Genaues sagen. Die Tatsache aber, dass die Metaphonie dort schwächer entwickelt ist als in den anderen russischlappischen Dialekten, lässt vermuten, dass die Metaphonie dort zumindest nicht von grösserer Bedeutung sein kann als im Skoltlappischen.

Der Wandel der morphologischen Methode in den lappischen Mundarten von agglutinierend $\mathrm{zu}$ fusionierend und fusionierend-symbolisch ist die Folgeerscheinung einer sehr komplizierten Reihe von Lautveränderungen. Die Funktion der einzelnen Lautwandel im Wandlungsprozess der morphologischen Methode ist jeweils verschieden. Es gibt Wandel, die an sich keinen morphologisch distinktiven Wechsel bewirken können sondern dazu die Voraussetzungen brauchen, die eine andere Lautveränderung schafft. Hierher gehören die Lautwandel, die zum Stufenwechsel und zum Metaphoniewechsel führen. Die Längung des $n$ zu $\grave{n}$ z.B. in der Form Part. Perf. *mënĕmĕ $>{ }^{*}$ mëñemĕ machte den Unterschied zwischen $n$ und $\grave{n}$ weder morphologisch noch phonologisch distinktiv, denn der Wandel trat immer und nur in der offenen Silbe ein. Nachdem aber die Apokope den Wandel *mĕnèmĕ $>{ }^{*}$ mĕnèm verursacht hatte, erhielt der Stufenwechsel sogleich eine morphologische Funktion, denn nur die starke Stufe trennte das Part.Perf. noch von der 1. Sg.Prs. mënèm, vgl. lp N mânâmmânnâm. Einige Lautveränderungen, wie die Apokope im vor- 
angehenden Beispiel, verursachen keinen paradigmatischen Wechsel sondern fördern nur das Distinktivwerden eines schon früher entstandenen Lautwechsels. Andere Lautwandel können gleichzeitig einen Wechsel verursachen und einen anderen Wechsel distinktiv machen. Das gilt z.B. für viele lappische Kontraktionen. So bewirkte die Kontraktion 1. Sg. Prät. *mènèjèm $>{ }^{*}$ mènim ( $>\operatorname{lp} \mathrm{N}$ mânnim) gleichzeitig einen paradigmatischen Wechsel in der 2. Silbe des Verbstamms (urlp. *měñè- $>\operatorname{lp} \mathrm{N}$ mânnấ-) und machte auch den Stufenwechsel distinktiv. Der Wechsel $\check{e} \sim i$ in der 2 . Silbe wurde seinerseits dann morphologisch distinktiv, als das Part.Perf.

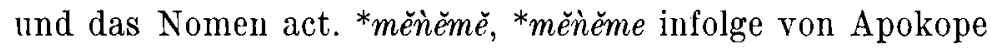
zu der Lautgestalt *mènèm (lp N mânnâm-mânnim) verkürzt waren.

Lautwechsel wurden veranlasst durch die metaphonischen und die zum Stufenwechsel und zum Strukturtypwechsel führenden Lautwandel sowie durch die İberdehnung. Als typisch begegnen sie im Inlautvokalismus und -konsonantismus, sie kömnen aber auch im Grenzvokalismus (lpN Nomen act. boattem - Part.Perf. boattam) in Frage kommen oder im Nebenkonsonantismus (lpSk. Nom.Sg. leǰlikaš - Gen., Akk.Sg., Nom.Pl. leכ̌lkląz). Distinktiv wurde der Lautwechsel durch den Schwund von Lauten im Wortauslaut und die Neutralisierung der Oppositionen im Grenz- und Suffixvokalismus oder -konsonantismus. Die gleichzeitig einen Wechsel bewirkenden und eimen anderen Wechsel distinktiv machenden Lautveränderungen erscheinen im Neben- und Grenzvokalismus, im Nebenkonsonantismus und in den Suffixen: es sind Kontraktion, Verschmelzung und verschiedene Wandel im System und in den Verhältnissen der Phoneme, Aufteilung, Zusammenfall und U̇berschneidung.

Betont werden muss, dass alle der behandelten Wandel in erster Linie phonetisch und phonologisch waren. Eine morphologische Funktion erhielten die durch diese Veränderungen bewirkten Wechsel deshalb, weil die Lautwandel, die die bereits existierenden Wechsel distinktiv machen, die Schlusslaute des Wortes und speziell die Suffixe betreffen und einen Verfall der Suffixe verursachen. Wenn die Suffixe ihre di- 
stinktiven Züge verlieren, geht ihre morphologische Funktion auf einen Wechsel im Stamminnern über. Die in den einzelnen Teilen des Stammes eingetretenen Lautwandel, die zu distinktiven Wechseln führten, haben sich verschieden ausgewirkt auf die morphologische Methode. Die Wandel im Inlautvokalismus und -konsonantismus führten zu rein im Innern des Stammes erscheinenden Wechseln, die in morphologischer Funktion typisch sind für die symbolische Methode. Die Lautwandel aber, die im Grenzvokalismus eintraten oder die den Grenzvokalismus und die suffixalen Elemente gemeinsam betrafen (z.B. die Kontraktion *mènèjĕm > $>{ }^{*}$ mènim), haben hauptsächlich zur fusionierenden Methode geführt. Ein in Kontraktions- und Verschmelzungsfällen entstandener Grenzvokal vertritt sowohl einen ursprünglichen Grenzvokal als auch ein Suffixelement. Auch morphologisch distinktive Wechsel des Grenzvokals - als Resultat der Metaphonie entstanden - wie lp N Nomen act. boattem - Part.Perf. boattam $\left(<{ }^{*}\right.$ prieme - * pritamĕ) sind vielleicht deskriptiv gesehen eher fusionierend, die Verbindung der Morpheme betreffend, als symbolisch.

2.3.2. Wechsel in Ableitungsfunktion. Wenn sich der Stamm des Grundwortes und der der davon gebildeten Ableitung in allen oder in einigen Formen des Paradigmas lediglich in ihrer inneren Struktur voneinander unterscheiden, ohne dass ein Ableitungssuffix sichtbar ist, haben wir die symbolische Derivationsmethode vor uns.

Obgleich der Symbolismus in der Flexionslehre des Lappischen eine beachtliche Stellung einnimmt, ist seine Bedeutung in der Ableitungslehre doch gering geblieben. Es wird angenommen, dass auch die Ableitungssuffixe im Frühurlappischen suffixal waren, welcher Zug sich in allen lappischen Dialekten gut erhalten hat. Die wenigen Fälle, wo das Ableitungssuffix geschwunden ist und seine Funktion auf einen Teil des Stammes übergegangen ist, haben im Prinzip dieselbe Geschichte wie die entsprechenden Veränderungen in der Flexion: zunächst hat sich ein Lautwechsel im Stamminnern ent- 
wickelt, dann ist das Ableitungssuffix geschwunden oder hat seine Distinktivität verloren. Da die Ableitungslehre für die Erforschung der veränderten morphologischen Methode des Lappischen nichts Neues bietet, was nicht schon aus der Behandlung der Flexionslehre hervorgeht, erachte ich es hier nicht für notwendig, die Funktion der Wechsel im Innern des Stammes in der Derivation des Lappischen eingehend zu beschreiben, sondern ich begnüge mich damit, Beispiele für jeweils typische Fälle anzuführen.

Die grösste Gruppe von Ableitungen, die in zahlreichen Formen des Paradigmas nur durch den Wechsel im Stamminnern von der entsprechenden Form des Grundworts unterschieden sind, stellen vermutlich die deverbalen, mit dem Suffix $j e$ gebildeten Verbalableitungen dar. Z.B. $\operatorname{lpN}$ al' lat 'sich erholen, wieder aufleben' vom Verb allet 'leben', 1. Sg. Prs. al'lam-alam, 2. cl'lak-alak, 3. cl'la-alla usw. Die für die Derivation distinktive Überdehnung ist die Folge des Schwundes eines zum Ableitungssuffix gehörenden $j$ und der darauf vollzogenen Kontraktion. Die Anzahl der Kontraktionsformen wechselt dialektweise etwas (s. genauer E. Itkonen MSFOu 79 S. 330-332). Auf Kontraktion beruht auch die symbolische Opposition zwischen der Deminutivableitung und dem Grundwort im Ostlappischen, die nur in solchen obliquen Kasus in Frage kommt, wo der gekürzte Stamm der Deminutivableitung verwendet wird, z.B. lpI (E. Itkonen) Lok.Sg. tālüst 'Haus' -- Dem.Lok.Sg. tālūst (<

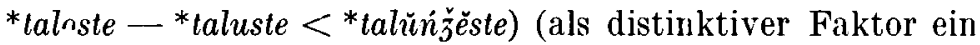
durch Metaphonie verursachter Wechsel).

Dass es in der Derivation weniger symbolische Methode gibt als in der Flexion, beruht vor allem auf der unterschiedlichen Stellung von Ableitungssilben und Flexionsendungen im Wort. Die stets am Ende des Wortes stehenden Flexionsendungen sind einem Schwund und anderen, die Distinktivität herabsetzenden Veränderungen mehr ausgesetzt als die Ableitungssuffixe, die fast immer im Inlaut vor der Flexionsendung stehen. Die Konjugation hat keine einzige Form, wo das Ableitungssuffix ursprünglich im Wortauslaut gestanden 
hätte, und die Deklination weist nur eine solche Form auf, den Nom.Sg. Somit kommt als Lautwandel, der einen derivativen Symbolismus hervorruft, fast ausschliesslich ein Schwund inlautender T'eile im Wort in Frage, wie er im Lappischen i.a. als Kontraktion auftritt. Eine zweite, in der Deklination vorkommende Möglichkeit ist, dass das nur aus einem Einzelkonsonanten bestehende Ableitungssuffix im Nom.Sg. im Auslaut geschwunden ist. Fälle dieser Art sind ans den mittleren und östlichen Dialekten des Lappischen hinreichend bekannt, doch geht es dabei meist um relativ unproduktive Ableitungstypen, wo das Grundwort als zweites Glied der Opposition nicht nachgewiesen werden kann, z.B. $\operatorname{lp} \hat{N} b \bar{a} n \hat{a}$ (<*penĕh) 'Hund', vuone (<*vonem) 'Schwiegermutter',

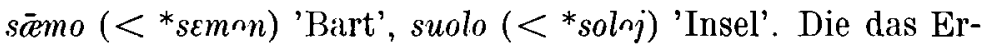
gebnis, das Objekt oder das Mittel einer Handlung ausdrükkenden deverbalen Nominalableitungen mit dem Suffix *ěk, die im Nom.Sg. keinen Suffixkonsonanten haben, stehen mit dem Stamm ihres Grundverbs in Opposition, z.B. čăla (<*čalĕk) 'Schrift' -- čallet 'schreiben', vgl. 3. Sg.Prs. čalla, 2. Sg.Imp. čâlě usw., äigâ 'Absicht' (<*ajaĕk) --ai'got 'beabsichtigen', vgl. 3. Sg.Prs. ai'go, 2. Sg.Imp. aigö usw. Wenigstens im Norwegischlappischen werden diese Ableitungen nur von den $e$ - und $o$-stämmigen Verben gebildet (Nielsen, Lærebok i lappisk I S. 193). Der Grenzvokal der Ableitungen ist wiederum stets $\hat{a}$, das in keiner Form eines $e$ - und $o$-stämmigen Verbs als Grenzvokal auftritt. Weil also der Wechsel des Grenzwokals $\hat{a}-e, \hat{a}-a, \hat{a}--o$ immer distinktiv ist zwischen der Ableitung und dem Grundwort, kann die Ableitungsmethode in diesem Fall eher als fusionierend denn als symbolisch bezeichnet werden. Relativ häufig tritt im Lappischen eine Fusion auf, wo das Ableitungssuffix oder ein Teil dessen den Auslautvokal des Grundstammes verdrängt hat, z.B. bâllo 'Furcht' (bâllât 'fürchten'), biw'do 'Fang, Jagd' (biu'det 'fangen, jagen'), suttiš 'leicht böse' (sut'tât 'böse werden'), mui'tâlit 'erzählen' (mui'tet 's. erinnern'), jâl'lot 'verrückt werden' (jâl'lâ 'verrückt') usw. 
3. DER UMFANG DER REDUNDANZ ALS REGULATOR DER MORPHOPHO NEMISCHEN VERÄNDERUNGEN IM LAPPISCHEN

In drei Phasen änderte das Lappische seine morphologische Methode und wurde von einer agglutinierenden zu einer fusionierend-symbolischen Sprache. Zuerst entstanden durch mechanische Lautwandel Allophonwechsel im Innern des Stammes. Zweitens kam es zu Lautwandeln, durch die die in der ersten Phase entstandenen Lautwechsel distinktiv wurden. Aus Allophonwechsel wurden Phonemwechsel. Drittens büssten die Suffixe ihre Distinktivität ein und die phonologisch distinktiven Wechsel im Stamminnern wurden morphologisch distinktiv. Unsere Untersuchung zeigt, dass die erste und zweite Phase Voraussetzung sind für die dritte, den Verfall und Schwund der Suffixe, doch kann ein Lautwandel mitunter gleichzeitig der zweiten und der dritten Phase angehören. Es lässt sich leicht beobachten, dass in den ostlappischen Dialekten, wo die Suffixe ihre Distinktivität am meisten verloren haben, auch die meisten Wechsel im Stamminnern vorhanden sind. Im Norwegisch- und Lulelappischen sind die Suffixe besser erhalten, doch gibt es dort auch weniger Wechsel im Innern des Stammes als im Ostlappischen. Im Südlappischen sind die Suffixe gut vom Schwund verschont geblieben, doch haben wir hier auch weniger morphologisch brauchbare Wechsel im Innern des Stammes als in den anderen lappischen Mundarten. Der Metaphoniewechsel ist zwar häufig, doch hatte er, wie auf den Seiten 291-294 ausgeführt, nicht die Voraussetzungen, sich zu einem zentralen morphologisch distinktiven Faktor zu entwickeln.

Ferner kann festgestellt werden, dass die Entsprechung zwischen dem Verfall der Suffixe und den Lautwechseln im Stamminnern nicht nur quantitativ sondern auch qualitativ ist. In den Dialekten, wo der Wechsel im Stamminnern am weitesten entwickelt ist, haben die Suffixe nicht nur am meisten an Distinktivität verloren, sondern die vor sich gegangenen Veränderungen stehen in Verbindung mit der Art der Wechsel im Innern des Stammes. So kommt der Verfall der 
Suffixe im Lule-, Norwegisch- und Inarilappischen in der Hauptsache als Schwund der Schlusslaute zum Ausdruck. Dies hängt damit zusammen, dass der in den betreffenden Dialekten vorherrschende Wechsel im Stamminnern der Stufenwechsel ist. Im Südlappischen, wo der Stufenwechsel fehlt, sind die suffixalen Elemente zwar erwartungsgemäss vom Schwund bewahrt geblieben, doch ist dafür das Vokalsystem der nichtersten Silben bis aufs äusserste vereinfacht, weil die Qualität der ursprünglichen Vokale der nichtersten Silben infolge der Metaphonie qualitätsmässig vorweggenommen wurde im Vokalismus der vorangehenden Silben, ja sogar auch im Konsonantismus. Hieraus folgte, dass der Metaphoniewechsel phonologisch und in gewissem Ausmass auch morphologisch distinktiv wurde. Im Russischlappischen herrscht sowohl ein alle Konsonanten betreffender Stufenwechsel als auch ein weitgehender Metaphoniewechsel; es gibt dort sowohl den Schwund von Lauten im Auslaut eines Wortes als auch die Vereinfachung des Vokalsystems der nichtersten Silben. Dies hat dazu geführt, dass der Stufenwechsel und teilweise auch der Metaphoniewechsel heute phonologisch sind und dass der Stufenwechsel und in einigen Fällen auch die Metaphonie eine wichtige morphologische Funktion besitzen.

Obgleich dem Verfall der Suffixe im Lappischen offenbar generell die Entwicklung von Lautwechseln im Innern des Stammes voranging, ist dies jedoch keine universelle Regel. Ebenso gut kann die Funktion des schwindenden Suffixes z.B. nur noch mit syntaktischen Mitteln ausgedrückt werden, wobei es auch zu einem typologischen Übergang kommt, diesmal von der synthetischen zur analytischen Methode. Das trifft ja bekanntlich z.B. für das Englische zu. Im Lappischen ist der Übergang zum Analytismus selten, doch lassen sich sporadische Belege finden. So hat sich z.B. die Opposition zwischen Dual und Plural im Russischlappischen mit Ausnahme des skoltlappischen Dialekts von Paatsjoki in der Konjugation und der possessiven Deklination ausgeglichen. $\mathrm{Ob}$ es sich dann um zwei oder mehrere Täter oder Besitzer handelt, muss jeweils irgendwie analytisch ausgedrückt werden, z.B. 
unter Verwendung eines personalpronominalen Subjekts oder Attributs. In den Personalpronomina hat sich die Opposition zwischen Dual und Plural nämlich erhalten.

Allgemein genommen ist die Entwicklung morphologisch brauchbarer Lautwechsel im Stamminnern also die Voraussetzung für den Verfall der Suffixe im Lappischen. Besteht nun zwischen diesen beiden Erscheinungen ein solcher kausaler Zusammenhang, dass der Reichtum an Lautwechseln im Innern des Stammes direkt zum Verfall der Suffixe führt? Es ist denkbar, dass die Opposition zwischen Formen des gleichen Paradigmas bereits in Stamm zum Ausdruck kommt, wenn sich in der Sprache aus irgendeinem Grund entsprechende Lautwechsel im Stamminnern entwickeln; die ursprünglich dem Suffix zukommende Information geht dann über auf ein Stammelement, das Suffix wird redundant, verliert seine distinktiven Besonderheiten und schwindet vielleicht schliesslich. Zunächst einmal ist zu untersuchen, was Information und Redundanz in einer natürlichen Sprache bedeuten.

3.1. Die Prinzipien der Informationstheorie, Entropie und Redundanz

Aufgestellt und entwickelt wurde die Informationstheorie hauptsächlich im Bereich der Kommunikationstechnik, sie lässt sich aber als allgemeine mathematische Theorie auch auf andere Kommunikationssysteme anwenden als die technischen im engen Sinne des Wortes. Auch die Sprache kann von der Informationstheorie her untersucht werden, was die Sprachwissenschaftler durch ihr Interesse dafür von Anfang an bezeugten. ${ }^{1}$

1 Vorgestellt wurde die Informationstheorie zuerst von C. E. Shannon in dem Aufsatz "A Mathematical Theory of Communication" (Bell System Technical Journal 27, 1948, S. 379-423,623-656) und bald danach ergänzt durch W. W. Weawer in der Arbeit "The Mathematical Theory of Communication" (Urbana, Illinois 1949). Sprachwissenschaftliche Anwendungen und Stellungnahmen erschienen im Laufe der Jahre reichlich, vor allem in den Vereinigten Staaten und in der Sowjetunion. Ich 
Nach der Informationstheorie hängt der Umfang der Information bzw. die Entropie für jedes Zeichen eines Symbolsystems ab von der Anzahl der verschiedenen Zeichen, ihrer relativen Frequenz und der Beschränkungen hinsichtlich der Verbindungen der Zeichen. Am grössten ist die Entropie dann, wenn alle Zeichen die gleiche Frequenz haben und wenn die Zeichen völlig unabhängig voneinander sind, so dass alle Kombinationen möglich sind. Diese sog. maximale Entropie $\left(\mathrm{H}_{\mathrm{o}}\right)$ wird ausgedrückt durch die Formel

$$
\mathrm{H}_{0}=\log _{2} \mathrm{n},
$$

wobei n die Anzahl der zum System gehörenden Zeichen bedeutet. In einer Sprache z.B., die 32 Phoneme hat, wäre die maximale Entropie $\log _{2} 32=5$. Dann würde jedes Phonem durchschnittlich ebenso oft auftreten und alle Phonemkombinationen wären möglich. Solche Bedingungen werden von keiner natürlichen Sprache erfüllt. Die Entropie verringern sowohl die verschiedenen Frequenzen der Phoneme als auch die Regeln im Hinblick auf die Verbindung der Phoneme miteinander. Wenn angenommen wird, dass den einzelnen Zeichen eine unterschiedliche Häufigkeitswahrscheinlichkeit zukommt, dass es aber keine Beschränkungen gibt in der Verbindung der Zeichen miteinander, erhält die Entropie den Wert

$$
\mathrm{H}_{1}=-\sum_{\mathrm{i}=1}^{\mathrm{n}} \mathrm{p} \text { (i) } \log _{2} \mathrm{p} \text { (i), }
$$

beziehe mich hier in der Hauptsache auf die folgenden Quellen: C. E. Shannon, Prediction and Entropy of Printed English (Bell System Technical Journal 30, 1951, S. 50-64); C. F. Hockett, Review of C. E. Shannon and W. Weawer's 'The Mathematical Theory of Communication' (Language 29, 1953, S. 69-93); G. A. Barnard, Statistical Calculation of Word Entropies for Four Western Languages (IRE Transactions on Information Theory IT-1, 1955, S. 49-53); G. Herdan, Language as Choise and Chance S. 162-197 (Groningen 1956), Quantitative Linguistics S. 173-177 (London 1964); E. V. Padučeva, Information Theory and the Study of Language (in dem Werk Exact Methods in Linguistic Research, hrsg. von O. S. Ahmanova, I. A. Mel'cuk, R. M. Frumkina und E. V. Padučeva, Übersetzung aus dem russ. Original, Berkeley and Los Angeles 1963); Р. Г. Пиотровский, Информационные измерения язына (Leningrad 1968). 
wo $n$ die Anzahl der einzelnen Zeichen wiedergibt und $\mathrm{p}(\mathrm{i})$ die Häufigkeitswahrscheinlichkeit des einzelnen Zeichens, wobei $\mathrm{i}$ die Werte $1,2, \ldots, \mathrm{n}$ erhält.

Berücksichtigt man die Abhängigkeit der aufeinander folgenden Zeichen voneinander, erhält man die bedingte Entropie. Zu ihrer Errechnung sind verschiedene Methoden entwickelt worden. Man kann u.a. erst die auf ein Zeichen entfallende Entropie in dem Fall errechnen, wo die Zeichen voneinander unabhängig sind $\left(\mathrm{H}_{1}, \mathrm{~s}\right.$. Formel 7$)$, und unter Verwendung dieses Resultats den Wert, wo die Beschränkungen hinsichtlich der Aufeinanderfolge zweier Zeichen berücksichtigt sind. Hierfür sind Frequenztabellen anzulegen von den möglichen Bigrammen, der Aufeinanderfolge zweier Zeichen. Wenn die Vorkommenswahrscheinlichkeit eines jeden Bigramms $p(i, j)$ bekannt ist, kann unter Anwendung von Formel (7) die auf das Bigramm entfallende Entropie $\left(\mathrm{H}^{2}\right)$ berechnet werden:

$$
\mathrm{H}^{2}=-\sum_{\mathrm{i}, \mathbf{j}}^{\mathrm{n}} \mathrm{p}(\mathrm{i}, \mathrm{j}) \log _{\mathbf{2}} \mathrm{p}(\mathrm{i}, \mathrm{j}) .
$$

Die Approximation der bedingten Fntropie des einzelnen Zeichens, wo die gegenseitige Abhängigkeit der Zeichen paarweise herücksichtigt ist $\left(\mathrm{H}_{\mathbf{2}}\right)$, erhalten wir, indem wir die Differenz zwischen $\mathrm{H}^{2}$ und $\mathrm{H}_{1}$ berechnen, also

$$
\mathrm{H}_{2}=\mathrm{H}^{2}-\mathrm{H}_{1} \text {. }
$$

In gleicher Weise wird aufgrund der Wahrscheinlichkeit des Vorkommens aller möglichen Belege von drei aufeinander folgenden Zeichen, der Trigramme $p(i, j, k)$ die Entropie des Trigramms $\left(\mathrm{H}^{3}\right)$ errechnet:

$$
\mathrm{H}^{3}=-\sum_{i, j, k}^{n} p(i, j, k) \log _{2} p(i, j, k),
$$

und weiter die Approximation der auf ein Zeichen entfallenden Entropie, wobei die gegenseitige Abhängigkeit der drei aufeinander folgenden Zeichen berücksichtigt ist $\left(\mathrm{H}_{3}\right)$

$$
\mathrm{H}_{3}=\mathrm{H}^{3}-\mathrm{H}^{2} \text {. }
$$


Das lässt sich so fortführen, bis die gegenseitige Abhängigkeit genügend vieler aufeinander folgender Zeichen berücksichtigt worden ist. Die Entropie eines einzelnen Zeichens in einem System, wo sich die gegenseitige Abhängigkeit der Zeichen über $m$ aufeinander folgender Zeichen hinaus erstreckt, ist

$$
\mathrm{H}_{\mathrm{m}}=\mathrm{H}^{\mathrm{m}}-\mathrm{H}^{\mathrm{m}-1} .
$$

Fndlich nähern wir uns einer Situation, wo die gegenseitige Abhängigkeit der Zeichen vollständig berücksichtigt ist. Dann nähert sich der Wert von $\mathrm{H}_{\mathrm{m}}$ jenem der wirklichen Entropie (H), die in dem diesbezüglichen System einem Zeichen zukommt. Je länger die Einheiten sind, über die sich die gegenseitige Abhängigkeit der Zeichen erstreckt, desto schwächer wird die Entropie.

Durch die relative Entropie $\left(\mathrm{H}_{\mathrm{rel}}\right)$ wird ausgedrückt, wie effektiv die Verwendung eines Zeichensystems vom Standpunkt der Entropie gesehen ist, d.h. in welchem Verhältnis die wirkliche Entropie zu der maximalen Entropie steht, die mit dem System erreichbar ist:

$$
\mathrm{H}_{\text {rel }}=\frac{\mathrm{H}}{\mathrm{H}_{\mathrm{o}}}
$$

( $\mathrm{H}_{\mathrm{o}}$ erhalten wir durch das Schema 6 S. 306).

Die Redundanz ( $\mathrm{R}$ ) ist dann

$$
\mathrm{R}=1-\mathrm{H}_{\text {rel }} \text {. }
$$

Zwischen der maximalen Entropie, der wirklichen Entropie, der relativen Entropie und der Redundanz bestehen also folgende Beziehungen:

1. Wenn die maximale Entropie wächst, d.h. wenn die Anzahl der Zeichen zunimmt und die wirkliche Entropie sich gleichbleibt, nimmt die relative Entropie ab und wächst die Redundanz.

2. Wenn die wirkliche Entropie wächst, d.h. wenn die Beschränkungen der Kombinierung der Zeichen abnehmen, und die maximale Entropie unverändert bleibt, dann wächst die relative Entropie und die Redundanz nimmt ab. 
Im Grenzfall, wo die Zeichen voneinander unabhängig sind und die Wahrscheinlichkeit ihrer Häufigkeit gleich ist, sind wirkliche und maximale Entropie gleich gross. Die relative Entropie ist dann 1 und die Redundanz $1-1=0$. Die theoretische obere Grenze der Redundanz ist 1 , was bedeutet, dass die Kombinierung der Zeichen vollständig im Voraus bestimmt ist, ohne die Möglichkeit einer Alternative. In einem solchen Fall ist die wirkliche und auch die relative Entropie 0 und die Redundanz $1-0=1$.

Relative Entropie und Redundanz werden oft in Prozenten ausgedrückt:

$$
\begin{aligned}
& \mathrm{H}_{\text {rel }}=100 \cdot \frac{\mathrm{H}}{\mathrm{H}_{\mathrm{o}}} \text { und } \\
& \mathrm{R}=100-\mathrm{H}_{\text {rel }} .
\end{aligned}
$$

3.2. Der Umfang von Entropie und Redundanz in den Sprachen

Es ist zu beachten, dass die Begriffe Information und Redundanz in mathematischer Bedeutung nicht das besagen, was man in der alltäglichen Rede darunter versteht. Im normalen Sprachgebrauch versteht man unter Informationsmenge oft die inhaltliche Aussage, den semantischen Gehalt eines Wortes, eines Satzes oder generell einer sprachlichen Darstellung. Die Informationsmenge bzw. die Entropie, wie die Informationstheorie sie definiert, ist lediglich eine Grösse, abgeleitet von der Häufigkeitswahrscheinlichkeit der im Kode begegnenden diskreten Symbole und deren Abhängigkeit voneinander, die nichts zu tun hat mit dem semantischen Wert der diesbezüglichen Symbole oder ihrer Verbindungen. Will man die Sprache nun von der Informationstheorie her behandeln, darf nicht vergessen werden, dass den Begriffen Informationsmenge und Redundanz nur der Inhalt zukommt, den die Informationstheorie ihnen gibt. Hierauf hat z.B. G. Herdan (Quantitative Linguistics S. 174-175) aufmerksam gemacht. Da ein geringes Mass an Information für ein Symbol eine grosse Häufigkeits- 
wahrscheinlichkeit bedeute, schreibt er, sei es leichter, ein Synbol mit geringer Information richtig einzuschätzen als ein solches mit hohem Informationsgehalt. Deshalb wird die Verständlichkeit einer Nachricht nicht sonderlich beeinträchtigt, wenn auch nicht alle Symbole ihr Ziel erreichen, nur die relative Entropie muss niedrig genug sein. Ist die relative Entropie aber gross, kann schon ein kleiner Fehler bewirken, dass die Nachricht nicht oder falsch verstanden wird. Ein hoher Informationsgehalt in der Sprache würde ferner nach Herdan einen Mangel an Strukturiertheit bedeuten, der eine Verwendung und ein Verständnis der Sprache praktisch ausschliesse. Ein niedriger Informationsgehalt steht wiederum in Verbindung mit der sprachlichen Struktur, dass sich nämlich die Symbole nach bestimmten Regeln miteinander verbinden und dass nicht alle Kombinationen möglich sind, was eine unbedingte Voraussetzung ist für das Gelingen sprachlicher Kommunikation. So kommt Herdan zu dem Schluss, dass das, was in der Informationstheorie Information ist, in der Sprache Mangel an Information sei, während die Redundanz der Informationstheorie sprachliche Strukturiertheit und Effektivität bedeute.

Herdans Gedankengang enthält eigentlich nur die den Linguisten von jeher bekannte Tatsache, dass die Sprache, will sie funktionieren, ziemlich viel Redundanz braucht. Und das deshalb, weil der Kommunikationskanal in der normalen Redesituation stets viel Störung enthält, das gerade mit Hilfe der Redundanz überwunden wird. Das Streben nach Ökonomie aber setzt dem Ausmass der Redundanz offenbar bestimnite Grenzen. Ausgangspunkt für die folgenden Überlegungen ist, dass das Ausmass der Redundanz reguliert wird durch eine optimierung der Effektivität und der ökonomie.

Die wirkliche Entropie für ein jedes Phonem in einer beliebigen Sprache unter Verwendung der Schemata 6--12 zu berechnen, ist eine nicht zu bewältigende Aufgabe. Die gegenseitige Abhängigkeit der Phoneme erstreckt sich nicht nur über ein Morphem, sondern da auch die Kombinierungsmöglichkeiten der Morpheme beschränkt sind, muss das ganze Wort berücksichtigt werden und da die Kombinierung von 
Wörtern durch ein syntaktisches Regelsystem reguliert wird, müsste man, um ein genaues Resultat zu erzielen, ausgehen von ganzen Sätzen oder lieber von noch längeren Einheiten. Eine Statistik der Häufigkeitswahrscheinlichkeiten langer Phonemketten verlangt die Analysierung einer ungeheuer grossen Menge von Text. E. V. Padučeva hält schon einen Text von 10000 Phonemen für unzureichend bei der Errechnung der Frequenzen der Kombinationen nur dreier Phoneme (Exact Methods in Linguistic Research S. 151). C. E. Shannon hat ein experimentelles Verfahren entwickelt, wonach die Grenzwerte, zwischen denen die wirkliche Entropie besteht, genügend genau definiert werden können, nicht ganz mühelos, aber doch leichter als nach dem oben geschilderten Rechnungsverfahren (Bell System Technical Journal 30 S. 54 -64). Das Verfahren beruht auf der Tatsache, dass alle Menschen zum Teil unbewusst über ein beträchtliches Wissen vom statistischen Bau ihrer Muttersprache verfügen. Die Versuchsperson kann den ersten Buchstaben eines willkürlich gewählten Textes raten. Rät sie falsch, darf sie so oft raten, bis sie den richtigen Buchstaben nennt. Danach errät sie den zweiten Buchstaben, dann den dritten usw. Der das Experiment ausführt, schreibt sich auf, wie oft jeder Buchstabe geraten wurde. Je mehr Information ein Buchstabe enthält, desto öfter muss natürlich aller Wahrscheinlichkeit nach geraten werden und umgekehrt. Anhand der Tabellen, die die Anzahl der Rateversuche an einem genügend grossen Material verzeichnen, lassen sich nach bestimmten mathematischen Verfahren die oberen und unteren Grenzen des Informationsgehaltes errechnen, die wiederum nach Schema 13 und 14 die oberen und unteren Grenzen für die relative Entropie und die Redundanz ergeben.

R. G. Piotrovski hat für fünf indoeuropäische und zwei türkische Sprachen die oberen und unteren Grenzen des Informationsgehalts und der Redundanz errechnet (Информационные измерения языка, S. 57-62). In Prozenten sehen die oberen und unteren Grenzen der Redundanz folgendermassen aus: 


$\begin{array}{ll}\text { Russisch } & 72,1-83,6 \\ \text { Polnisch } & 74,7-85,0 \\ \text { Englisch } & 71,9-84,5 \\ \text { Französisch } & 70,6-83,4 \\ \text { Rumänisch } & 72,1-85,0 \\ \text { Aserbaidschanisch } & 65,2-79,0 \\ \text { Kasachisch } & 70,9-81,0\end{array}$

Die Werte sind nach schriftlichen Texten mit dem Buchstaben als Einheit errechnet und stimmen also offenbar mindestens für das Englische und Französische nicht ganz überein mit den entsprechenden Werten der Phonemsprache. Trotzdem kann man feststellen, dass die Redundanz sprachenweise relativ wenig wechselt. Die untere Grenze schwankt zwischen 65,2 und 74,7, die obere zwischen 79,0 und 85,0. Piotrovski hat die Grenzen von Informationsgehalt und Redundanz in den fünf indoeuropäischen Sprachen auch in den einzelnen Stilschichten berechnet, der gesprochenen Sprache, der Belletristik und der Sachprosa. Im Englischen z.B. erhielt er die Werte 69,4-81,2 für die Redundanz der gesprochenen Sprache, 77,1-86,5 für die Belletristik und $82,9-$ 92,1 für die Sachprosa. Die Unterschiede sind beachtlich. Im Rumänischen aber sehen die entsprechenden Zahlen so aus: $74,2-85,9,73,8-83,8$ und $74,4-85,9$. Die Unterschiede in der Redundanz der Stilschichten scheinen also einzelsprachlich zu schwanken.

Auch andere Forscher haben festgestellt, dass die zwischensprachliche Differenzen im Umfang der Redundanz relativ gering sind. Nach Charles Hockett liegt die am Sprechtempo errechnete Redundanz in allen phonologischen Systemen bei 0,50 (Manual of Phonology, Baltimore 1955, S. 218). Der so errechnete Redundanzwert ist als solcher nicht vergleichbar mit den für die Häufigkeitswahrscheinlichkeiten der Phoneme berechneten Werten, doch zeigt Hocketts Angabe, falls sie zutrifft, dass die verschiedenen Verfahren im Hinblick auf die Standardredundanz zum selben Ergebnis führen. In diese Richtung weist ferner die Tatsache, dass sich die Anzahl der einzelnen Phoneme in den Sprachen umgekehrt proportional zur Länge der Morpheme verhält. Als Grenzfälle wer- 
den das Hawaische, wo es nur 13 Phoneme gibt und dessen Morpheme nur sehr selten einsilbig, meist zweisilbig und oft mehrsilbig sind und einige kaukasische Sprachen angeführt, in denen es über 70 Phoneme gibt und wo praktisch jedes aufeinander folgende Phonem im Text ein Morphem darstellt (Hockett, A Course in Modern Linguistics, New York 1960, S. 93). Da sich der durchschnittlich auf ein Phonem entfallende Informationsgehalt umgekehrt proportional zur Anzahl der Phoneme verhält, ist in einer phonemreichen Sprache in einem kurzen Morphem ebenso viel Information enthalten wie in einer phonemarmen Sprache in einem langen Morphem. Wenn die Morpheme einer phonemreichen Sprache durchschnittlich gleich lang wären wie die einer phonemarmen Sprache, wäre auch die Redundanz einer phonemreichen Sprache grösser.

Grob gesehen ist die Redundanz direkt proportional zur Voraussagbarkeit des Textes. Diese Voraussagbarkeit lässt sich mit einem sehr einfachen Verfahren messen. Eine Versuchsperson soll die Phoneme eines ihr unbekannten Textes erraten, vom ersten angefangen. Rät sie falsch, wird ihr das richtige Phonem gesagt und sie darf weiter raten. Rät sie richtig, bestätigt der Experimentierende ihr die Richtigkeit der Antwort und sie errät dann das nächste Phonem, danach das dritte usw. Dieses Verfahren unterscheidet sich von dem auf den Seiten $311-312$ genannten darin, dass jedes Phonem nur einmal geraten wird. Der prozentuale Anteil der richtig geratenen Phoneme an der Gesamtzahl der im Text enthaltenen Phoneme drückt die Voraussagbarkeit aus. S. Shannon a.a.O. 54, Badučeva Exact Methods...S. 154. Der auf diese Weise errechnete Prozentsatz der Voraussagbarkeit steht dann dem Wert der wirklichen Redundanz sehr nahe, wenn sich die Redundanz ihren Grenzwerten nähert, in Prozenten 0 oder 100. Wenn die Redundanz des Kodes 100 betrüge und die Entropie also 0, dann wäre auch die Voraussagbarkeit eine vollständige und die Versuchsperson würde alle Symbole raten oder besser wissen. Das Rate-Experiment führte dann zu einem Resultat, das genau dem Wert der Redundanz entspräche. Wenn aber die Redundanz des Kodes 0 wäre und die relative Entropie 100, könnte die Versuchs- 
person nur durch Zufall richtig raten und der Prozentsatz der richtig geratenen Symbole würde sich 0 nähern. Die Redundanz der Phonemsysteme in den natürlichen Sprachen ist weit entfernt von diesen Grenzwerten, doch kann der Voraussagbarkeitsprozentsatz auf jeden Fall als eine Art grober approximativer Wert der Redundanz gelten.

Ich habe derartige Experimente angestellt anhand der finnischen, estnischen, norwegischlappischen und ungarischen Hochsprache, jeweils mit Versuchspersonen, die die betreffende Sprache vollständig beherrschten. Als Einheit gilt der Buchstabe, da aber die Orthographien der genannten Sprachen fast phonologisch sind, dürften die Ergebnisse nicht viel von jenen abweichen, die zustande gekommen wären, wenn die Texte phonologisch bezeichnet gewesen wären und die Testpersonen die Phoneme erraten hätten. Im Ungarischen wurde ein Zugeständnis an das phonologische System gemacht, wo die ein Phonem bezeichnenden Bigramme folgendermassen durch Phonemzeichen ersetzt wurden: $c s>\check{c}, s z>s$, $z s>z, g y>d, l y>j, n y>n, t y>t$. Das $s$ der Orthographie wurde durch $s$ bezeichnet. Die Resultate gehen aus den Tabellen 7 und 8 hervor.

Die Orthographien berücksichtigen nicht alle distinktiven Oppositionen. Die grösste Abweichung vom phonologischen System zeigt in dieser Hinsicht das Estnische, dessen Rechtschreibung jene phonologische Quantitätskorrelation unberücksichtigt lässt, die z.B. in den Wortpaaren Gen.Sg. lin̆naPart.Sg. liǹna 'Stadt', Nom.Sg. hōne - Gen.Sg. hône 'Gebäude' (Orth. linna - linna, hoone - hoone) überall begegnet, ausser wenn als Konsonantismus nach der betonten Silbe ein Geminatatenuisklusil steht (Gen.Sg. vakkka-Part.Sg. valkka, Orth. vaka - vakka 'Schachtel; Scheffel'). Der niedrige Prozentsatz der Voraussagbarkeit im Estnischen, $62 \%$, kann teilweise hierauf beruhen. Die funktionale Belastung der Quantitätskorrelation im Estnischen ist beachtlich, und die Quantitätsstruktur des Vokalismus der ersten Silbe und des Stammkonsonantismus enthält oft eine solche Information, die die Wahrscheinlichkeit des richtigen Erratens des letzten Teiles des Wortes und der syntaktischen Funktion erhöht. 
Tabelle ?

\begin{tabular}{|c|c|c|c|c|c|}
\hline Sprache & \begin{tabular}{|c|} 
Ver- \\
suchs- \\
person
\end{tabular} & Text & $\mathrm{n}$ & $\mathbf{x}$ & $\mathrm{P}$ \\
\hline \multirow[t]{3}{*}{ Finnisch } & A & Eino Leino, Pakinat I, 1960, S. 59 & 500 & 367 & 73 \\
\hline & A & $\begin{array}{l}\text { Juhani Aho, Papin rouva, ab } \\
\text { Kap. } 24\end{array}$ & 372 & 257 & 69 \\
\hline & B & Eino Leino a. a.o. S. 107 & 368 & 253 & 69 \\
\hline \multirow[t]{2}{*}{ Estnisch } & C & $\begin{array}{l}\text { Tuglas, Valik kriitilisi töid, Tal- } \\
\text { linn } 1959 \text {, S. } 131\end{array}$ & 421 & 258 & 61 \\
\hline & $\mathrm{C}$ & $\begin{array}{l}\text { Mart Raud, Teosed III, Tallinn } \\
1964 \text {, S. } 99\end{array}$ & & 269 & 62 \\
\hline \multirow[t]{2}{*}{ Lappisch } & $\mathrm{D}$ & 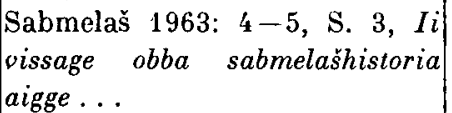 & 449 & 310 & 69 \\
\hline & $\mathbf{E}$ & $\begin{array}{l}\text { Lars Hætta ja Anders Bær, } \\
\text { Mui'talusat, Oslo 1958, S. 51 } \\
\text { Móricz Zsigmond, Riportok, Bu- }\end{array}$ & 848 & 590 & 70 \\
\hline \multirow{4}{*}{$\begin{array}{l}\text { Unga- } \\
\text { risch }\end{array}$} & $\mathrm{F}$ & dapest 1958, S. 173 & 217 & 133 & 61 \\
\hline & $\mathrm{F}$ & $-"-$ S. 179 & 298 & 188 & 63 \\
\hline & $\mathrm{F}$ & $\begin{array}{l}\text { Bálint György, A toronyór visz- } \\
\text { szapillant, Budapest } 1966, \text { S. 421 }\end{array}$ & 373 & 267 & 72 \\
\hline & G & $\begin{array}{l}\text { Diószegi Vilmos, Sámánok nyo- } \\
\text { mában Szibéria földjén, Buda- } \\
\text { pest } 1960, \mathrm{~S} .72\end{array}$ & 503 & 355 & \\
\hline
\end{tabular}

Tabelle 8. Ergebnisse sprachenweise zusammengerechnet

\begin{tabular}{|c|c|c|c|}
\hline Sprache & $\mathrm{n}$ & $\mathrm{x}$ & $\mathrm{P}$ \\
\hline Finnisch & 1240 & 877 & 21 \\
\hline Estnisch & 856 & 527 & 62 \\
\hline Lappisch & 1297 & 900 & 69 \\
\hline Ungarisch & 1391 & 943 & 68 \\
\hline
\end{tabular}

$\mathrm{n}=$ Anzahl der Buchstaben im Text, $\mathrm{x}=$ Anzahl der richtig geratenen Symbole, $\mathrm{P}=$ Anzahl der richtig geratenen Symbole in Prozenten $=$ Voraussagbarkeitsprozent 
Die Wortgrenze wurde bei dem Experiment als Buchstabe gezählt. In der Praxis enthält die Rede ja stets eine Information von den Wortgrenzen. Die Grenzsignale sind meistens suprasegmental und kommen zum Ausdruck in der Qualität, der Quantität, dem Intonationsverlauf usw. der segmentalen Phoneme. Wenn nach den allgemeinsten Ansichten der taxonomischen Phonologie die Junktur als Phonem betrachtet wird, entspricht die Berücksichtigung der Wortgrenze als ein Buchstabe der Stellung der Junktur als Phonem in jener Phonemkette, den der geschriebene Versuchstext repräsentiert. Das gilt zumindest für die hier behandelten Sprachen, wo eine Bindung von Wörtern zu Einheiten ohne Junktur etwas Seltenes ist. (Zum Charakter der Junktur i.a. und besonders vom Standpunkt der finnischen Sprache s. Terho Itkonen MSFOu 138,1 S. 24-29 und die dort genannte Literatur.) In der generativen Phonologie wird wiederum angenommen, dass die Wortgrenzen primär in der phonologischen Oberflächenstruktur auftreten (s. Chomsky - Halle, The Sound Pattern of English, New York 1968, S. 366-). Die Satzzeichen blieben systematisch unberücksichtigt.

Das verwendete experimentelle Verfahren enthält eine Reihe von Fehlerquellen. Erstens sind auch die besten schriftsprachlichen Orthographien nicht völlig phonologisch und zweitens kann auch die sprachliche Begabung der Versuchsperson wie die Schwierigkeit des Textes auf das Resultat einwirken. Als Text wurde möglichst einheitliche Normalprosa ausgewählt. Für das Ungarische wurde ausserdem getestet, wie weit stilistische Unterschiede in der Redundanz zum Ausdruck kommen. Die Riportok von Móricz stehen für umgangssprachlichen Stil mit seinen Besonderheiten, die übrigen Texte für normale Sachprosa. Die Redundanz der Textproben von Móricz beträgt 61 und $63 \%$, die der Sachprosastücke 72 und $71 \%$. Das Ergebnis stimmt überein mit den Beobachtungen von Piotrovski, wonach die Redundanz der Sachprosa in den fünf untersuchten indoeuropäischen Sprachen am höchsten ist; am niedrigsten ist die Redundanz der gesprochenen Sprache im Russischen, Englischen und Französischen und die der Belletristik im Polnischen und Rumänischen (Информационные измерения языка, S. 59). 
Es lässt sich ferner fragen, ob das Phonem überhaupt die geeignete Einheit ist, an der Informationsgehalt und Redundanz errechnet werden. Der Phonembegriff ist in der letzten Zeit von den Anhängern der Transformationsgrammatik kritisiert worden. Bei der sog. phonologischen Komponente der generativen Grammatik wird ausgegangen von der relativ abstrakten Oberflächenstruktur, gebildet durch eine Kette von Formativen, und mit Hilfe von Regeln kommt man zur phonetischen Vertretung, ohne dass die Phonemebene in irgendeiner Phase in Erscheinung tritt. Beachtenswert ist jedoch, dass der Untersuchungsgegenstand der generativen Grammatik die sprachliche Kompetenz ist (Chomsky-Halle a.a.O. 3), während die Informationstheorie die Sprache als Kode auffasst, wodurch Information übertragen wird. Primär handelt es sich um die gesprochene Sprache, um Übertragung von Information vom Sprecher auf den Hörer. Luftdruckwellen, in Intensität und Dichte wechselnd, bilden dabei die physikalischen Elemente des Kodes. Sie stellen ein Kontinuum dar, worin sich mit physikalischen Mitteln keine genau umgrenzten Einheiten, "Laute", feststellen lassen. Erst wenn er die Sprechsignale empfängt, gliedert der Hörer den Lautwellenstrom in getrennte, einander folgende Einheiten. Zweifellos geschieht diese Aufteilung in Einheiten gerade mittels der sprachlichen Kompetenz, doch der wirkliche Charakter derselben ist wohl noch nicht endgültig erkannt. Wieviel Meinungsverschiedenheiten über den Begriff des Phonems selbst auch existieren mögen - deutlich ist doch, dass die diskreten Einheiten dem Phonem nahekommen (s. Hocket Language 29 S. 69-84, Padučeva Exact Methods ... S. 144-). Aber auch die phonetische Vertretung nach der generativen Grammatik ist nicht das Ergebnis einer Registration aller in der Sprache beobachteten Züge, sondern eher eine Auslegung des Sprecher-Hörers, die zahlreiche physikalische Besonderheiten der Sprache unberücksichtigt lässt, wie die Stimmhöhe und -qualität des Sprechers, die auf der Artikulationsbasis beruhenden Besonderheiten, die Übergänge zwischen Vokal und Konsonant usw. (Chomsky - Halle a.a.O. 293-). Es ist schon zeimlich viel Quantisierung darin enthalten, wenn man die phonetische Vertretung auffasst als zweidimen- 
sionale Matrix, deren Kolumnen den aufeinander folgenden phonetischen Einheiten entsprechen und deren Waagerechte die einzeInen phonetischen Besonderheiten zeigen. M.E. gibt es jedoch kein objektives Kriterium, aufgrund dessen die verschiedenen phonetischen Einheiten einer Sprache aufgezählt werden könnten. Der funktionale Aspekt muss auf die eine oder andere Weise unbedingt berücksichtigt werden, wobei man sich dann bereits der phonologischen Betrachtungsweise nähert.

Es ist wohl mit aller Wahrscheinlichkeit anzunehmen, dass die an phonologischer 'Transkription oder fast phonologischer Orthographie errechneten Prozente der Voraussagbarkeit zumindest untereinander vergleichbar sind und dass die darin iuftretenden Abweichungen von den wirklichen Redundanzwerten der Phonemsysteme gleichgerichtet und ungefähr gleichgross sind. Somit stimmen die obigen Resultate gut mit dem überein, was früher hinsichtlich der Universalität der Redundanz angenommen wurde. Verschiedene Texte aus verschiedenen Sprachen mit verschiedenen Versuchspersonen ergaben Ergebnisse, die sich zwischen 61 und $73 \%$ bewegen, iuf einem relativ schmalen Riaum also.

Die Konstanz der sprachlichen Redundanz ist an sich nur verständlich. Die physiologischen und psychischen Voraussetzungen des Menschen, die Sprache als Kommunikationsmittel zu verwenden, dürften überall in der Welt ungefähr gleich sein. Auch die äusseren Verhältnisse, in denen eine Sprache zur Anwendung kommt, dürften zwischen den einzelnen Sprachbereichen im Durchschnitt nicht viel differieren, was in Einzelfällen natürlich in starkem Ausmass der Fall sein kann. Ein bestimmter Umfang von Redundanz ist notwendig, damit die Information über einen Kanal, dessen verschiedene Abschnitte unterschiedliche Störungen, Geräusche, enthalten, an ihr Ziel gelangt. Das in der Sprache vorhandene Streben nach Wirtschaftlichkeit - von Zipf in seinen Untersuchungen über den geringsten Aufwand überzeugend dargestellt (Human Behavior and the Principle of Least Effort, Cambridge, Mass. 1949) - vermindert die Redundanz, die wiederum erhöht wird durch das Streben nach sicherer Über- 
mittlung der Information, trotz Störungen. Diese entgegengesetzten Kräfte bewirken, dass die Redundanz nicht darüber hinaus ansteigt, was zur Überwindung normaler Störungen nötig ist, aber auch nicht so weit sinkt, dass die Kommunikation darunter leidet (vgl. Hockett, A Manual of Phonology S. 218). Es handelt sich also um eine Art automatischen Mechanismus, durch den unter möglichst geringem Aufwand eine möglichst grosse Effektivität garantiert werden soll. Da sich die psychophysischen Eigenschaften des Menschen und die äusseren störenden Faktoren im Laufe der Jahrtausende offenbar praktisch nicht verändert haben und da auch lokale Schwankungen - statistisch gesehen - kaum auftreten, wäre es durchaus nicht verwunderlich, wenn die Redundanz in allen Sprachen der Welt gleich wäre. Für die Verifikation dieser These wären natürlich Untersuchungen vieler verschiedener Sprachen nötig sowie die Klärung der Frage, ob die eventuellen intersprachlichen Redundanzunterschiede eine Entsprechung haben in physiologischen und psychischen Eigenschaften der Sprecher oder in Umweltsfaktoren. Für unsere augenblickliche Untersuchung brauchen wir nicht so weit zu gehen. Es genügt die Feststellung der Wahrscheinlichkeit, dass die Redundanz einer Sprache unverändert bleibt, falls es in den Umweltverhältnissen der Sprecher dieser Sprache oder in sprachlich wichtigen psychischen und physiologischen Eigenschaften nicht zu wesentlichen Veränderungen kommt. Wir werden davon ausgehen können, dass sich die Lappen oder ihre Umwelt seit der frühurlappischen Zeit nicht so verändert haben, dass die Redundanz der Sprache dadurch vermindert oder erhöht worden wäre.

3.3. Die Konstanz der Redundanz und das Distinktivwerden der Lautwechsel im Innern des Stammes in den lappischen Dialekten

Wie auf S. 308 festgestellt wurde, nimmt die Redundanz zu mit wachsender maximaler Entropie, was wiederum eine direkte Folge ist von der Vermehrung der Anzahl der Sym- 
bole, die in dem betreffenden Kommunikationssystem verwendet werden. In den lappischen Dialekten, wo die Wechsel im Innern des Stammes zu einem bedeutsamen morphologischen Faktor wurden, überall also ausser im Südlappischen, ist die Anzahl der Phoneme nach der urlappischen Zeit beträchtlich angestiegen. Das Frühurlappische besass wahrscheinlich zumindest die folgenden Konsonantenphoneme der $x$-Reihe:

$$
\begin{array}{llclllll}
p & & & v & & & & m \\
t & s & (\vartheta) & \delta & c & l & r & n \\
& & \left(\delta^{\prime}\right) & c & & & n \\
k & & & j & & & & \eta
\end{array}
$$

In der $x x$-Reihe begegneten schon damals wenigstens $p p, t t$, $k k$ und $c ̌ c \quad\left(=t t^{s}\right) .{ }^{1}$ Konsonantenphoneme gab es also insgesamt 19-21. An Vokalen gab es im Frühurlappischen die folgenden 12:

$\begin{array}{lllll} & & & \bar{\imath} & \bar{u} \\ \ell & 0 & & \\ e & \hat{o} & & \bar{e} & \bar{o} \\ \varepsilon & n & & \\ \ddot{a} & a & & \end{array}$

(s. Erkki Itkonen, Suomen Akatemia puhuu, Porvoo 1968, S. 54).

Schon in der urlappischen Zeit wurde die Geminatenreihe so ergänzt, dass die meisten Einzelkonsonanten am Ende der urlp. Zeit paarweise mit einer Geminate auftraten. Stark erweitert wurde der Phonembestand aber erst, als der Stufenwechsel phonologisch wurde. Prinzipiell muss dies mit dem Eintreten einer Situation geschehen sein, dass die starke und die schwache Stufe nicht mehr in kombinatorischer Distribution standen. Denkbar wäre, dass die ersten Kontraktionen eine solche Situation herbeigeführt haben. Die ältesten lappischen Kontraktionen gehen sicher auf das Urlappische zurück (s. S. 237, 246). Als neben dem erwartungsgemäss schwachstufigen Typus *mĕnĕm trotz der geschlossenen

${ }^{1}$ Auf $i j$ weist lpN ag'gja 'Grossvater, Alter' $\sim$ fi. äijä hin. 
Silbe das starkstufige ${ }^{*}$ mĕǹim $\left(<{ }^{*}\right.$ měnèjĕm) entstand, war der Stufenwechsel nicht mehr komplementär im ehemaligen Sinne. Die ältesten Kontraktionen vollzogen sich früher als irgendein Schwund eines Auslauts im Laufe der Eigenentwicklung des Lappischen, denn wir haben kein einziges sicheres Beispiel für einen urlp. Schwund im Auslaut. ${ }^{1}$ Eine Einschränkung gibt es jedoch im Hinblick auf den Anteil der Kontraktion an der Phonemisierung des Stufenwechsels. Es ist nämlich wahrscheinlich, dass die Kontraktionsvokale ursprünglich quantitätsmässig und vielleicht auch qualitätsmässig abwichen von den nichtkontrahierten Vokalen. So konnten die stark- und die schwachstufigen Konsonanten weiterhin gegenseitige Allophone bleiben. Die Voraussetzungen für die starke Stufe änderten sich nur insofern, als die starke Stufe nun sowohl vor der offenen Silbe als auch vor der geschlossenen Silbe mit einem Kontraktionsvokal auftrat. In einigen Formgruppen aber, die als alte Kontraktionsfälle zu gelten haben, wie in der 1 . und 2 . Sg. Prät., ist der Kontraktionsvokal schon frühzeitig mit dem entsprechenden nichtkontrahierten Vokal zusammengefallen, wie aus der sehr einheitlichen Vertretung in allen Dialekten zu schliessen ist (vgl. E. Itkonen MSFOu 88 S. 7, 117-118).

Als der Stufenwechsel phonologisch wurde, erhöhte sich die Anzahl der Konsonantenphoneme mit einem Schlag auf das Doppelte. Anzumerken ist jedoch, dass - wäre der phonetische Stufenwechsel vom Typ $\grave{x} x: x x$ und $\grave{x}: x-$ die schwache Stufe der Geminaten und die starke Stufe der Einzelkonsonanten spätestens mit der Phonemisierung des Stufenwechsels wahrscheinlich zusammenfallen würden, wobei die Wechselverhältnisse $\grave{x} x: \grave{x}$ und $\grave{x}: x$ wären, worauf auch der Stand der heutigen Dialekte mit Stufenwechsel zurückzuführen ist (vgl. Bergsland Studia Septentrionalia II S. 15-, E. Itkonen $F U F 29$ S. 278-279, Ravila $F U F$ 33 S. 318-). Schon die Prager Schule stellte die Existenz dreier phonologischer Quantitätsstufen in Frage, und die Quantität ist ja auch nicht der einzige distinktive Faktor für die lappischen

1 Vgl. jedoch S. 279. 
Stufen $x^{\prime} x, x x$ und $x$, sondern der Intensitätsverlauf ist auch beteiligt und ausserdem verbindet sich mit dem Quantitätswechsel im Konsonantismus ein Wechsel der Quantitätsstruktur des ganzen Wortes (s. Ravila Vir. 1961 S. 345350). In einfacher phonologischer Darstellung lassen sich die Quantitätsverhältnisse jedoch so darstellen, dass der Konsonantismus drei Quantitätsstufen aufweist, wobei einige Quantitätsverhältnisse des Vokalismus und der Intensitätsverlauf von der Quantität des Konsonantismus abhängige Begleiterscheinungen sind. Das gilt z.B. für die Orthographie von Nielsen und auch für die heutige westnorwegischlappische Orthographie (die von Bergsland und Ruong). Wenn man berücksichtigt, dass $* \delta$ und ${ }^{*} \delta$ ' im Inlaut zusammengefallen sind (vielleicht schon vor dem Urlappischen) und dass auch das anlautende $* \delta^{\prime}$ mit anderen Phonemen verschmolzen ist und dass $\breve{s}$ (und dialektweise $\vartheta$ ) als neue Konsonanten zum System hinzukamen, erhalten wir für die mittellappischen Dialekte 17-18 konsonantische Qualitäten. Da von allen drei phonologisch relevante Quantitätsstufen begegnen (oder QuantitätIntensitätsstufen), erhielte man auf diese Weise bereits insges. 51-54 konsonantische Phoneme. Im Finnmarklappischen gehören noch die stimmlosen $h, J, M, N, f, R$ und $L$ zum System, von denen die drei letztgenannten in allen Quantitätsstufen begegnen. Es entstand ausserdem die Mediareihe $b, d, g, z, \check{z}$, ebenfalls für alle Quantitätsstufen. Die längeren Tenuisreihen des Urlappischen sind zu Konsonantenverbindungen geworden $\left(* \dot{k}>h k,{ }^{*} \grave{k} k>\bar{h} k,{ }^{*} \dot{c}>h \check{c},{ }^{*} \dot{c} \check{c}>\right.$ $\bar{h} c$ usw.), so dass sie nur in einer Quantität begegnen (dialektal in zwei: $* \grave{k}>h k, * \grave{k}_{k}>h \bar{k}$ usw.). Vor einem Tenuis im Inlaut begegnet stets ein stimmloser Übergangslaut, der als $h$ gedeutet werden kann (Nielsen, Lærebok i lappisk I S. 2123), z.B. ač'če 'Vater' = aȟče, giettâ 'Hand' = kiehtâ, bal'ka 'Lohn' = pailhka, Gen. balka = palhka usw. Die aspirierten Klusile im Wortanlaut sind wiederum als Verbindung eines Klusils mit $h$ zu deuten, z.B. tëggja 'Tee' = theäddâ. Das Verhältnis zwischen den Tenuis- und Mediareihen ist komplementär, denn die Tenuis begegnen nur neben einem anderen stimmlosen Konsonanten und die Media wiederum stets 
in stimmhafter Umgebung. Der Palatalspirant $g$ ist das Allophon eines Palatalklusils. Phonologisch gesehen gibt es im Norwegischlappischen also eine einzige in drei Quantitätsstufen vorhandene Klusil- und Affrikatenreihe. $l$ tritt nur in den zwei längeren Quantitätsstufen auf. Lassen wir die hauptsächlich in ganz jungen Lehnwörtern und in Expressiva begegnenden $f, J, M, N, R$ und $L$ weg und berücksichtigen nur die Entwicklung im Phonembestand urlappischer Herkunft, erhalten wir für die kurze Quantitätsreihe die folgenden 18 Konsonantenphoneme:

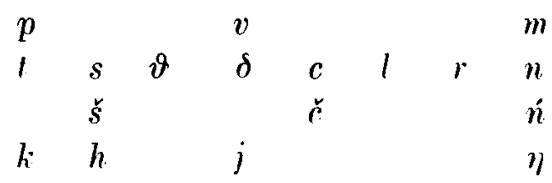

In der langen und gedehnten Reihe begegnen zusätzlich $d$ und $l$, in der gedehnten fehlen dagegen $j$ und $h$. Das $j$ der langen Reihe erscheint in Konsonantenverbindungen, z.B. ajke = Nielsen aige. Als Anzahl der Konsonantenphoneme ergibt sich somit $18+20+18=56$. Die Konsonantenverbindungen lassen sich alle als Kombinationen dieser Phoneme deuten.

Der Vokalphonembestand des Norwegischlappischen ist schon deshalb weitaus schwerer zu beschreiben, weil es da offenbar mehr interdialektale Unterschiede gibt als im Konsonantismus. Die wirtschaftlichste Lösung, für die meisten Dialekte geeignet, könnte vielleicht folgendermassen aussehen:

$\begin{array}{cccccc}\check{\imath} & & \breve{u} & i & & u \\ \check{e} & & \check{o} & e & & o \\ & \hat{a} & & & a & \\ & \check{a} & & & & \end{array}$

Diese elf Phoneme entsprechen den Graphemen der Orthographie von Nielsen wie folgt:

$i \quad=$ kurzes, nichtwechselndes $i$ : vivvâ 'Schwiegersohn', lokkim 'ich las',

$\breve{u} \quad=$ kurzes, nichtwechselndes $u$ : gullât 'hören', ọlmuš 'Mensch', 
$\check{e} \quad=$ kurzes $\check{e}$ in gleicher Silbe: boadĕ 'komm!', vuoggjëlit 'losfahren',

$\check{o} \quad=$ kurzes, nichtwechselndes $\varrho$ der 1. Silbe: jọkkâ' Fluss', kurzes ŏ der gleichen Silbe : muttó 'aber', goarŏ 'nähe',

$\hat{a}=\hat{a}: j a ̂ k k e$ 'Jahr', dollâ 'Feuer',

$\check{a} \quad=$ kurzes $\breve{a}$ der gleichen Silbe: mannă-âkke 'Kindheit', laebă 'sind' (Du.),

$i=$ in der 1 . Silbe $i e \sim i$ und in nichtersten Silben $e$ : giettâ 'Hand', čirruk 'sie weinen', sadne 'Wort', gulâimek 'wir hörten',

$u=$ in der 1 . Silbe $u o \sim u$ und in der gleichen Silbe $o:$ buollet 'es brennt', būlii 'es brannte', dallo 'Haus',

$e \quad=$ in der ungleichen Silbe $a \sim e: l a i^{\prime} k \hat{a}$ 'Spiel', lei'kii 'zum Spiel',

$o \quad=$ in der ungleichen Silbe $o a \sim g:$ boatta 'er kommt', bộttiom 'ich kam',

$a=a:$ manna 'Kind'.

Unter den oben genannten Bedingungen lassen sich für das Norwegischlappische insgesamt 67 Phoneme feststellen. Zweifelsohne kann man auch andere Deutungen bringen, vielleicht auch solche, die "ökonomisch" bedeutend günstiger sind. Wenn man sich aber z.B. für den Konsonantismus mit zwei Quantitätsstufen begnügt, muss man entsprechend Vokalphoneme hinzufügen. Der Klarheit halber sei noch erwähnt, dass die suprasegmentalen Phoneme uns diesmal nicht interessieren, denn vom Standpunkt der Informationstheorie ist die Rede aufzufassen als eine linear fortlaufende Reihe von aufeinander folgenden Symbolen (vgl. Hockett Language 29 S. 84). Wir stellen fest, dass die Anzahl der Phoneme im Norwegischlappischen gegenüber dem Frühurlappischen mehr als das Doppelte beträgt, für welch letzteres wir 32 Phoneme annahmen (s. S. 320). Die maximale Entropie des frühurlp. Phonemsystems $\mathrm{H}_{0}=\log _{2} 32=5$, im gegenwärtigen Finnmarklappischen $\mathrm{H}_{0}=\log _{2} 67=6,07$. Indem der Stufenwechsel phonologisch wurde und die Geminatareihe ergänzt wurde, erhöhte sich also die maximale Entropie von 5 auf ungefähr 6 . Wie sich die wirkliche Entropie 
gleichzeitig änderte, lässt sich unmöglich genau abschätzen. Ausser von der Anzahl der Phoneme hängt ihr Wert ja auch $a b$ von ihren Häufigkeitswahrscheinlichkeiten und den Begrenzungen ihrer Kombinationen. Die Häufigkeitswahrscheinlichkeit neuer Phoneme der $x x$-Reihe war anfangs gering, weshalb die Frequenzverteilung innerhalb des neuen Phonemsystems ungleichmässig wurde. Mit der Phonemisierung des Stufenwechsels bildeten die starke und die schwache Stufe zunächst nur in wenigen Fällen phonologische Oppositionen, wie im folgenden genauer dargestellt wird. Meistens standen sie noch in kombinatorischer Distribution. Trotz des beachtlichen Anstiegs der Zahl der Phoneme kam es also nicht zu einer nennenswerten Vermehrung der Kombinationsmöglichkeiten der Phoneme. Im Gegenteil: jedes dem Stufenwechsel unterliegende, aus dem Frühurlappischen stammende Phonem war zwar durch zwei neue Phoneme vertreten, die Kombinationsmöglichkeiten aber waren beachtlich geringer als die des Stammphonems, da das eine weiterhin hauptsächlich nur vor der offenen und das andere vor der geschlossenen Silbe auftrat. Aufgrund des Gesagten dürfte es als wahrscheinlich gelten können, dass es in der wirklichen Entropie zu keinen bedeutenden Veränderungen kam, auf keinen Fall zu einem auch nur annähernd so grossen Umschwung wie in der maximalen Entropie. In der folgenden summarischen Veranschlagung nehmen wir an, dass die wirkliche Entropie unverändert geblieben ist.

Schema 13 (S. 308)

$$
\mathrm{H}_{\mathrm{rel}}=\frac{\mathrm{H}}{\mathrm{H}_{\mathrm{o}}}
$$

lässt erkennen, dass die relative Entropie dann gesunken wäre. Bezeichnen wir die relative Entropie des ursprünglichen Systems von 32 Phonemen mit $\mathrm{H}_{\text {rel } 1}$ und die relative Entropie nach der Phonemisierung des Stufenwechsels und der Ergänzung der Geminatareihe mit $\mathrm{H}_{\text {rel 2. Die Werte der rela- }}$ tiven Entropie in den einzelnen Phasen wären dann

$$
\mathrm{H}_{\text {rel } 1}=\frac{\mathrm{H}}{5} \text { und } \mathrm{H}_{\text {rel } 2}=\frac{\mathrm{H}}{6}
$$


Da $\mathrm{H}$ als Konstante angenommen wird, erhalten wir

$$
\mathrm{H}_{\mathrm{re1} 2}=\frac{5}{6} \mathrm{H}_{\mathrm{rel} 1}
$$

mit anderen Worten: Phonemisierung des Stufenwechsels und Ergänzung der Geminatareihe hätten die relative Entropie um ein Sechstel herabgesetzt. Dies hätte einen entsprechenden Anstieg in der Redundanz bewirkt, denn $\mathrm{R}=1-\mathrm{H}_{\text {rel }}$ (Schema 14, S. 308). Da Redundanz und relative Entropie in der Sprache offenbar konstant zu bleiben bestrebt sind, brauchte man für das Anwachsen der Anzahl der Phoneme irgendeine Gegenwirkung, um die Verminderung der relativen Entropie zu verhindern. Schema 13 (oben) zeigt, dass die relative Entropie konstant bleibt, falls die wirkliche Entropie $(\mathrm{H})$ mit dem Anwachsen der maximalen Entropie $\left(\mathrm{H}_{0}\right)$ im gleichen Verhältnis zunimmt. Die einzige Möglichkeit, die wirkliche Entropie mit Zunahme der Phoneme zu erhöhen, besteht in einer Vermehrung der Kombinationsfähigkeiten der Phoneme (s. S. 308, Regel 2). Auf den lappischen Stufenwechsel angewendet bedeutet das, dass die Voraussetzungen für den Stufenwechsel in den meisten Fällen noch erhalten waren, nachdem die ersten Lautveränderungen, die zur Phonemisierung des Stufenwechsels führten, wahrscheinlich Kontraktionen und in der Folge Ausgleich der Differenz zwischen Kontraktionsvokalen und nichtkontrahierten $\mathrm{V}_{0}$ kalen, eingetreten waren und die Zahl der Phoneme gleichzeitig stark zugenommen hatte. Die funktionale Belastung der neuen Phonemoppositionen war gering. Auch wenn das Verhältnis *mĕnĕm : *mĕǹim (< *mĕñèjĕm) 'ich gehe : ich ging' schon entstanden war, wo die starke Stufe vor einer geschlossenen Silbe auftreten konnte, trat doch weiterhin überwiegend die starke Stufe vor der offenen und die schwache Stufe vor der geschlossenen Silbe auf (*měǹemĕ 'gegangen': *mĕnĕm). Die Kombinationsmöglichkeiten der Phoneme waren immer noch an die ehemaligen Regeln gebunden, mit Ausnahme der wenigen Fälle, wodurch der Stufenwechsel phonemisiert worden war. Am wirkungsvollsten liessen sich die Kombinationsmöglichkeiten vermehren, indem man den Stufenwechsel 
möglichst unabhängig machte vom offenen oder geschlossenen Charakter der nachfolgenden Silbe. Der in den lappischen Stufenwechseldialekten begegnende ausgedehnte Schwund von auslautenden Vokalen und Konsonanten sowie die in diesen Dialekten nach dem Urlappischen erfolgten Kontraktionen und Lautverschmelzungen in nichtersten Silben haben grösstenteils als Ausdruck der Tendenz zu gelten, die Gebundenheit der phonologischen Quantitätsstufen an die Umgebung zu verringern. Eine Folgeerscheinung dieser Prozesse ist es, dass wir in den heutigen Stufenwechseldialekten bei den Quantitätsstufen des Konsonantismus kaum irgendeine Abhängigkeit von der Umgebung feststellen können. Somit ist die eine erhöhte Redundanz verursachende Wirkung des vermehrten Phonembestands aufgehoben und die Redundanz hat wahrscheinlich ungefähr denselben Wert wieder, den sie vor der Phonemisierung der Quantitäten besass.

Geht man von einer solchen Phonemanalyse aus, dass die Geminaten als Verbindung zweier in der Qualität gleicher Konsonanten aufgefasst werden, ist die Zunahme der Phoneme vom Frühurlappischen bis zu den heutigen Stufenwechseldialekten nicht so gross wie oben dargestellt. Vorauszusetzen sind jedoch zwei Quantitätsstufen, $x$ und $\grave{x}$, deren letztere

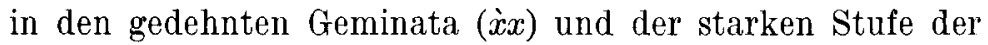
Konsonantenverbindungen $(\grave{x} y)$ begegnet. Dieser mit der Phonemisierung des Stufenwechsels erfolgte Anstieg der Zahl der Phoneme erhöht die maximale Entropie, jedoch nicht so stark wie dann, wenn alle distinktiven Quantitätsstufen als selbständige Phonemeinheiten aufgefasst werdelı. Wenn wir aber nun die Geminaten als Konsonantenverbindungen betrachten, dann wächst mit der Phonemisierung des Stufenwechsels und der Ergänzung der Geminatareihe die Anzahl der Phonempaare. Es entstehen die völlig neuen Phonempaartypen $\grave{x} x$ und $\grave{x} y$ und die Anzahl der Paare vom Typ $x x$ steigt. Die maximale Entropie der Phonempaare nimmt zu, da aber der Stufenwechsel unserer Vermutung nach anfangs nur in wenigen Fällen distinktiv war und die Kombinationsmöglichkeiten der Phonempaare nicht nennenswert vermehrt wurden, bleibt sich die wirkliche Entropie un- 
gefähr gleich, während die relative Entropie sinkt und die Redundanz steigt. So kommt man offenbar zu einem ungefähr gleichen Wachstum der Redundanz unabhängig davon, wie die Quantitätsstufen des Konsonantismus phonologisch gedeutet werden.

Nun kann die Frage gestellt werden, ob die Verminderung der relativen Entropie um ein Sechstel genügt, jenen Prozess auszulösen, der in der Hauptsache die morphologische Struktur des Lappischen von agglutinierend in fusionierend-symbolisch verwandelte. Die Antwort hierauf hängt wesentlich davon ab, wie weit die aufgrund der Häufigkeitswahrscheinlichkeit der Phoneme errechnete Redundanz überhaupt schwanken kann. Es muss betont werden, dass hierzu noch nicht genügend genaue Angaben vorliegen. In den von Piotrovski untersuchten Sprachen liegt die Schwankung der unteren Grenze der Redundanz bei $65,2-74,7 \%$ bzw. 9,5 Prozenteinheiten, die Schwankung der oberen Grenze bei $79,0-85,0 \%$ bzw. 6 Prozenteinheiten. Liesse man das Aserbaidschanische mit seinen abweichend niedrigen Werten weg, beliefe sich die untere Grenze nur noch auf 4,1 Prozenteinheiten und die obere auf 4,0 Prozenteinheiten. Für das Finnische, Estnische, Lappische und Ungarische schwankt die Voraussagbarkeit zwischen 62 und 71 , d.h. sie liegt bei 9 Prozenteinheiten. Hätte beim Test eine völlig phonologische Transkription verwendet werden können, wo auch alle distinktiven Quantitätsoppositionen des Estnischen berücksichtigt worden wären, wäre der Prozentsatz der Voraussagbarkeit für das Estnische wahrscheinlich gestiegen und die Schwankung wäre somit zusammengeschrumpft. Sowohl die Untersuchungen von Piotrovski als auch meine eigenen Experimente im Zusammenhang mit dieser Arbeit haben übereinstimmend bewiesen, dass die Stilschichten einen wesentlichen Einfluss ausüben auf die Redundanz. Bei den von Piotrovski untersuchten Sprachen wies das Französische die grössten Differenzen auf mit einer unteren Grenze der Redundanz von 83,9\% im Stil der Sachprosa und 68,5\% in der Alltagsrede, einem Unterschied von 15,4 Prozenteinheiten also; die obere Grenze liegt für die Sachprosa bei $90,4 \%$ und für die Alltagsrede bei 
$82,9 \%$, so dass der Unterschied 7,5 Prozenteinheiten ausmacht (Информационные измерения языка S. 59). Innerhalb einer Sprache kann die Redundanz also je nach Stilschicht und offenbar auch Sprechsituation beträchtlich schwanken, während verglichen damit die intersprachlichen durchschnittlichen Differenzen kleiner bleiben. Es lässt sich schwer sagen, inwieweit die Ergebnisse hinsichtlich der intersprachlichen Differenzen von zufälligen Faktoren beeinflusst wurden, doch dürfte klar sein, dass die Unterschiede eher kleiner als grösser würden, könnte man die in der Orthographie vorhandenen Abweichungen vom phonologischen System eliminieren. Wenn wir annehmen, dass der Prozentsatz der Voraussagbarkeit im Lappischen, 69 (s. Tabelle 8, S. 315), der tatsächlichen statistisch errechenbaren Redundanz nahekommt und der approximale Wert der relativen Entropie somit $31 \%$ beträgt, und weiterhin annehmen, dass diese Zahlen in grossen Zügen auch für das Urlappische galten, hätte eine Verminderung um ein Sechstel für die relative Entropie ungefähr 5 Prozenteinheiten ausgemacht. Wenn der Phonembestand also nach dem Frühurlappischen in der oben geschilderten Weise ohne nennenswerte Vermehrung der Kombinationsmöglichkeiten angewachsen wäre, dann wäre die relative Entropie auf $26 \%$ zurückgegangen und die Redundanz oder richtiger die Voraussagbarkeit wäre auf ca. $74 \%$ angestiegen. Dieser Wert muss insofern als beachtlich hoch gelten, als er bei meinen eigenen Testen kein einziges Mal erreicht wurde. Falls eine Erhöhung der Redundanz um ca. 5 Prozenteinheiten im Lappischen sehr schnell und ohne verhältnisbedingte Notwendigkeit eingetreten wäre, hätte eine derartige Schwankung wohl das Gleichgewicht zwischen Klarheit und Wirtschaftlichkeit zu stark zu ungunsten der Wirtschaftlichkeit beeinträchtigt. So hat denn auch offenbar das auf die erhöhte Anzahl von Phonemen zurückgehende Ansteigen der Redundanz als Gegengewicht jene Veränderungen hervorgerufen, die unter Herabminderung der Redundanz den Phonembestand der nichtersten Silben zum Verfall brachten, welche Erscheinung typisch ist für alle lappischen Dialekte mit Ausnahme des Südlappischen. 
Inwieweit die obigen Vermutungen zutreffen, kann folgendermassen getestet werden. Nehmen wir an, dass in den lappischen Texten beim Test für die Voraussagbarkeit die suffixalen Phoneme hinzugefügt werden, die nach dem Frühurlappischen geschwunden sein sollen. Diese Situation läge ja vor, wenn es keine Lautveränderungen gegeben hätte, die die Stellungsbedingtheit der Phoneme herabgesetzt hätten; dieser Stand herrscht ja heute noch weitgehend im stufenwechsellosen Südlappisch. Der Passus kuovtte maฑimuš vassam jâgi aigge 'im Laufe der beiden letztvergangenen Jahre' z.B. würde folgendermassen verändert (Zusätze in Klammern)

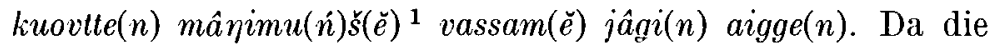
Versuchsperson alle Formen im ursprünglichen Text zu identifizieren versteht, würde sie nach eingehendem Studium des Systems alle hinzugefügten Phoneme richtig erraten können; der Text enthielte nunmehr die rekonstruierten urlappischen Suffixe, wäre aber sonst der gleiche. Mit anderen Worten: die Ergänzungen würden keine neue Information bringen. Der Prozentsatz der Voraussagbarkeit würde dann natürlich ansteigen. Der Anstieg müsste ungefähr übereinstimmen mit dem durch die erhöhte Anzahl der Konsonantenphoneme bewirkten Zunehmen der Redundanz, also ca. 5 Prozenteinheiten ausmachen. Da die hinzuzufügenden suffixalen Elemente völlig redundant sind, ist keine Versuchsperson nötig, sondern man kann die vermuteten urlappischen Suffixelemente nachträglich dem verwendeten Text einfügen und dann den Prozentsatz der Voraussagbarkeit errechnen. Die Zahl der falschen Voraussagen bleibt sich gleich, die der richtigen steigt entsprechend der höheren Anzahl von Buchstaben im Text. Tabelle 9 zeigt das Ergebnis vom vervollständigten Text verglichen mit dem vom ursprünglichen (vgl. Tabelle 7, S. 315).

Das Resultat von 4,3 Prozenteinheiten Anstieg in der Voraussagbarkeit entspricht relativ gut der oben anhand erhöhter maximaler Entropie ermittelten Zunahme der Redundanz

1 Anstelle von $\check{s}$ wäre eigentlich $\stackrel{\zeta}{\zeta}$ zu rekonstruieren. Diese Veränderung würde jedoch nichts an der Anzahl der Phoneme im Wort ändern. 
Tabelle 9

\begin{tabular}{|c|c|c|c|c|c|c|c|c|}
\hline \multirow{2}{*}{$\begin{array}{c}\text { Ver- } \\
\text { suchsp. }\end{array}$} & \multicolumn{3}{|c|}{ Urspr. Text } & \multirow{2}{*}{$\begin{array}{l}\text { Hinzu- } \\
\text { gefügte } \\
\text { Buch- } \\
\text { staben }\end{array}$} & \multicolumn{3}{|c|}{ Ergänzter Text } & \multirow{2}{*}{$\begin{array}{c}\text { Anstieg } \\
\text { von } \mathrm{P} \text { in } \\
\text { Prozent- } \\
\text { einh. }\end{array}$} \\
\hline & n & $\mathbf{x}$ & $\mathbf{P}$ & & $\mathrm{n}$ & $\mathbf{x}$ & $\mathrm{P}$ & \\
\hline D & 449 & 310 & 69,0 & 71 & 520 & 381 & 73,3 & 4,3 \\
\hline $\mathrm{E}$ & 848 & 590 & 69,5 & 142 & 990 & 732 & 73,9 & 4,4 \\
\hline Insges. & 1297 & 900 & 69,4 & 213 & 1510 & 1113 & 73,7 & 4,3 \\
\hline
\end{tabular}

um ca. 5 Prozenteinheiten. Die Übereinstimmung der mit zwei verschiedenen Methoden erzielten Ergebnisse beweist die Richtigkeit unserer Hypothese vom Anstieg der Redundanz im Lappischen und den als Gegengewicht dazu entwickelten, die Redundanz herabsetzenden Lautveränderungen.

Durch das Streben nach Aufrechterhaltung des Gleichgewichts zwischen relativer Entropie und Redundanz erklärt sich die Tatsache, dass die Wörter in den Stufenwechseldialekten durch den Schwund der Auslautphoneme und durch Kontraktion gekürzt wurden. Gleichzeitig wird aber auch die Frage beantwortet, weshalb die Morphologie in den Stufenwechseldialekten fusionierend-symbolisch wurde. Die Information, die mit dem Schwinden der Auslautphoneme des Wortes auf den Stammkonsonantismus überging, war morphologisch, denn die geschwundenen Phoneme waren suffixal. Somit wurde der Stufenwechsel, nunmehr phonologisch geworden, ein wichtiger Träger morphologischer Information.

Der bisherigen Darstellung liegt die Annahme zugrunde, dass zunächst ein Lautwandel nötig war, wahrscheinlich eine Kontraktion, damit der Stufenwechsel phonologisch wurde, und erst in diesem Stadium wurde die Redundanz so gross, dass eine Gegenwirkung zwecks Herabsetzung derselben aufkam. Eine andere Möglichkeit dürfte es kaum geben, wenn wir daran festhalten, dass für die Sprache als Kommunikationsmittel gerade die Phoneme jene diskreten Symbole darstellen, aus denen sich der Kode am einen wie am anderen 
Ende des Kanals zusammensetzt. Näher untersucht werden muss aber nun die Phase, wo der Stufenwechsel phonologisch wurde. Wie Ravila in seiner Arbeit über den lappischen Stufenwechsel festgestellt hat, gibt es keine scharfe Grenze zwischen phonetischem und phonologischem Stufenwechsel ( $F U F$ $33 \mathrm{~S} .288$ ). Der phonetische oder vielleicht eher der allophonische Stufenwechsel kann sehr unterschiedlich sein, abhängig davon, ein wie grosser Unterschied phonetisch zwischen der starken und schwachen Stufe besteht. Wenn sich die schwachund die starkstufige Variante im quantitativen Stufenwechsel so wenig voneinander unterscheiden, dass die Wahrnehmung des Unterschieds genauer Betrachtung bedarf, erkennen auch die Sprecher den Stufenwechsel nicht und er hat keine linguistische Bedeutung. Wird der Quantitätsunterschied aber gross genug, dass er für jeden leicht wahrnehmbar ist, und wenn noch eins der Allophone zusammenfällt mit einem zu einem anderen Phonem gehörenden Allophon (z.B. fi. aitta 'Speicher': Gen.Sg. aitan, aita 'Zaun': Gen.Sg. aidan), dann hört der Sprecher die Vertreter der schwachen und starken Stufe deutlich als verschiedene "Laute", auch wenn sie der deskriptiven Distributionsanalyse noch weiterhin als zum selben Phonem gehörig betrachtet werden können.

Eine derartige Situation entwickelte sich im Lappischen spätestens damals, als die schwache Stufe der Reihe $x \boldsymbol{x}$ und die starke Stufe der Reihe $x$ zusammenfielen, was geschehen konnte, bevor der Stufenwechsel phonologisch wurde, was aber spätestens sofort danach eintreten musste (vgl. S. 321322). In dem Stadium, da der Unterschied zwischen starker und schwacher Stufe deutlich wahrnehmbar geworden war, musste der Unterschied zwischen der schwachen Stufe der Reihe $x x$ und der starken Stufe der Reihe $x$ entsprechend kleiner werden. Als das Ohr nicht mehr imstande war, diesen Unterschied wahrzunehmen, hatte sich der Zusammenfall vollzogen. Geschah dies, bevor der Stufenwechsel phonologisch geworden war, mussten sich die Glieder des dreistufigen Quantitätssystems (oder Quantitäts-Qualitätssystems) von Konsonanten mit bereits vorhandener Quantitätskorrelation genügend voneinander unterscheiden, und die Sprecher muss- 
ten die Unterschiede auch erkennen, da sie nun unter bestimmten Bedingungen eine distinktive Funktion besassen. Vor der offenen Silbe stand $\grave{x} x$ in Opposition zu $\grave{x}$, z.B. urlp.

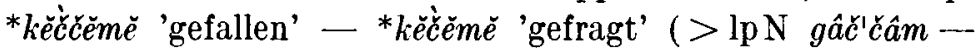
gấżâm), vor der geschlossenen Silbe stand $\dot{x}$ in Opposition zu $x$, z.B. *kěčèm 'ich falle' — *kěžèm 'ich frage' (>lpN gấčââm - gâă $\tilde{a} a m)$. Die mittlere Quantitätsstufe konnte sowohl vor der offenen als auch vor der geschlossenen Silbe auftreten. Am Ende der urlappischen Zeit, wo die schwache Stufe der Reihe $x x$ wohl zusammenfiel mit der starken der Reihe $x$, ging die Quantitätskorrelation offenbar schon über die Klusile und Affrikaten hinaus. Hierauf weist der Tatbestand hin, dass es im Lappischen eine Reihe von Wörtern gibt, deren Stammkonsonantismus in allen Dialekten eine andere Geminate als einen Klusil oder eine Affrikate aufweist und die also geminiert aufs Urlappische zurückgehen, z.B. Tän. (Lagercrantz) $p^{\prime}$ ’ĕäp p mà 'Speise', Malå (Schlachter) beäb'moo, N (Nielsen)

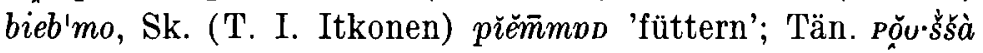
'Hinterraum im Zelte', Malå båssjoo, N boaš'šso, Sk. pọ̆ă̌šs, Kld. (T. I. Itkonen) pųašs Mer. (Lagercrantz) ка̀̀лииo 'Stirn, Kopffell', Malå gàlloo, N

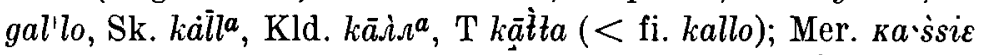

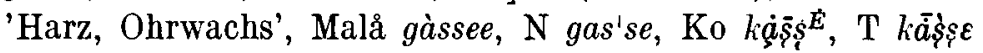
usw.

Da in einem grossen Teil des Konsonantismus die Quantitätsunterschiede somit berücksichtigt werden mussten, erkannte man natürlich auch bei den Konsonanten, die am Ende der urlappischen Zeit noch keine Quantitätskorrelation hatten, das Vorhandensein eines Unterschiedes zwischen starker und schwacher Stufe, auch wenn er noch nicht phonologisch war. Das Quantitätssystem war aber bereits reif zur Übernahme einer phonologischen Belastung. Quantität (und Qualität) des Stammkonsonantismus lieferten in Wirklichkeit bereits Information über den offenen oder geschlossenen Charakter der folgenden Silbe und oft auch darüber, ob ein grammatisches Morphem mit dem Stamm verbunden war und wenn, dann welches. Da die syntaktischen Regeln die Möglichkeiten des Auftretens der einzelnen Formen stark ein- 
schränkten, konnte der Hörer in vielen Situationen vielleicht lediglich aufgrund der Quantitätsstruktur des Wortstammes mit Sicherheit erfahren, um welche Form es sich handelte. Wesentlich für die Information ist, dass der vor dem Suffix stehende Teil des Wortes dem Suffix gewissermassen ganz oder teilweise seine Funktion raubte. So hatte man den eigenartigen Punkt erreicht, dass distributionsmässig phonologisch irrelevante Züge in Wirklichkeit distinktiv waren, während die Phoneme der nichtersten Silben mehr oder minder redundant geworden waren. Die starke und die schwache Stufe waren nun zu einer Art potentieller Phoneme geworden, die zwischen ihnen bestehenden Unterschiede waren wahrnehmbar und in der linearen Kommunikation hatten sie ihre Funktion, ihrer Distribution nach aber waren es noch Allophone. Dieses Stadium hat als Wendepunkt zu gelten auf dem Wege zur Phonemisierung des Stufenwechsels. Vom Standpunkt der Informationstheorie lässt sich die Sprechsituation so skizzieren, dass der Hörer dabei einen Kode empfängt, der aus aufeinander folgenden Symbolen zusammengesetzt ist. Der Empfänger erhält die Symbole einzeln, erst allmählich bildet sich eine Gesamtheit, die das Verständnis der Nachricht ermöglicht. In einer so beschaffenen Sprache, als welche wir das Urlappische in seinem letzten Stadium oben schilderten, ist es dann für den Hörer wichtig, ob auf den betonten Vokal des Wortes eine kurze, halblange oder lange Konsonantenquantität folgt, da er hieraus auf den Schlussteil des Wortes schliessen kann. Falls bereits im Urlappischen die Quantitätsstruktur das ganze Wort und höchstens einen dreisilbigen Takt betraf und ein Wechsel des Intensitätsverlaufs damit verbunden war, bekam der Hörer in vielen Fällen schon mit der ersten Silbe eine ganze Reihe von Information, die ursprünglich den letzten Silben des Wortes vorbehalten war. Von der Informationstheorie aus haben wir also die Quantitäts- (und Intensitäts-)unterschiede in einer derartigen Situation als distinktiv zu werten. Dies berechtigt uns dazu, bei der Berechnung von Entropie und Redundanz die stark- und schwachstufigen Varianten der Konsonanten in der letzten Phase des Urlappischen als selbständige funktionale Einhei- 
ten zu behandeln, Phonemen gleichgestellt. Für diese Einheiten verwenden wir im folgenden die Bezeichnung $Q$ u a s ip h o n e m.

Erkennen wir das Quasiphonem an als informationsmässig funktionale Einheit, dann können wir die Erweiterung des Phonembestands der lappischen Stufenwechseldialekte, den Verfall der nichtersten Silben und die Veränderung der morphologischen Methode unter einem etwas neuen Gesichtspunkt betrachten. Es braucht nicht mehr angenommen zu werden, dass ein Lautwandel, z.B. die Kontraktion, den Stufenwechsel erst phonologisch machen musste und dass erst die dadurch erfolgte Zunahme der Phoneme den Anstieg der Redundanz bewirkte. Da die Quasiphoneme bereits über die Struktur der nachfolgenden Silbe informierten, kann die Phase, wo der Stufenwechsel quasiphonemisch wurde, als entscheidend angesehen werden: die Sprecher bemerkten die Unterschiede zwischen starker und schwacher Stufe sowie die darin enthaltenen Hinweise auf die Struktur der nächsten Silbe. Zugegeben - dieses Stadium genan zu definieren, ist nicht einfach, und das Phänomen ist eher ein psychologisches denn ein linguistisches, was allerdings die Bedeutung des Allophonwechsels innerhalb der zur Phonemisierung führenden Entwicklung nicht herabmindert. Denkbar ist also, dass die erhöhte Anzahl von Quasiphonemen die Redundanz vermehrte, infolgedessen es dann zu Lautveränderungen kam, welche die Kombinierungsmöglichkeiten der Quasiphoneme vermehrten, wie Kontraktion, Schwund der im Auslaut des Wortês befindlichen Laute und verschiedene Verschmelzungen. So wurde der Stufenwechsel auch hinsichtlich seiner Distribution phonologisch sowie ebenfalls morphologisch distinktiv.

Es scheint, als hätte das Südlappische den Stufenwechsel verlorèn, ehe die starke und schwache Stufe zu selbständigen Phonemen oder Quasiphonemen wurden. E. Itkonen hat aufgrund einiger Konsonantenverhältnisse, die Bergsland im Südlappischen feststellte, angenommen, der quantitative Stufenwechsel habe auch im Lappischen ursprünglich den Typưs $\grave{x} x: x x, \grave{x}: x$ vertreten (Bergsland Studia Septentriona- 
lia II S. 15-, E. Itkonen FUF 29 S. 278-279). Danach könnte man annehmen, dass im Südlappischen nie ein Stufenwechsel vom Typus $\grave{x} x: \grave{x}, \grave{x}: x$ vorhanden gewesen sei, sondern dass sich der letztgenannte Wechseltypus in den nördlicheren Dialekten erst entwickelte, nachdem sich das Südlappische oder dessen älteste Komponente bereits vom Urlappischen getrennt hatte. Wir gingen oben davon aus, dass der Zusammenfall der schwachen Stufe der Reihe $x x$ mit der starken Stufe der Reihe $x$ zu den Grundvoraussetzungen für die Phonemisierung des Stufenwechsels gehört. Wir können hier weder prinzipiell noch praktisch die Frage lösen, ob in einem Wechselsystem von vier Quantitätsstufen die Unterschiede zwischen den einzelnen Stufen so gross sein konnten, dass der Stufenwechsel auch nur eine quasiphonemische Funktion hatte. Jedenfalls ist es ganz folgerichtig, dass der Stufenwechsel in einem vierstufigen Wechselsystem leichter schwinden kann als in einem dreistufigen. Das Fehlen des Stufenwechsels im Südlappischen und die gut erhaltenen Phoneme im Wortauslaut wären somit völlig verständlich.

Ravila hat sich dahingehend geäussert, dass die heutige südlappische Vertretung auch von den späturlappischen Wechselverhältnissen $\grave{x} x: \grave{x}, \grave{x}: x$ her erklärbar sei (FUF 33 S. 318-321). Er gründet seine Auffassung in erster Linie darauf, dass die Funktion der $\dot{x}$ - Quantität ungeachtet der phonetischen Gleichheit der schwachen Stufe der Reihe $x x$ und der starken Stufe der Reihe $x$ eine andere war, je nachdem, welche Reihe $\grave{x}$ vertrat. Zweifellos lässt sich das Fehlen des Stufenwechsels im Südlappischen auch auf dieser Basis erklären. Wenn Ravilas Erklärung vom Standpunkt des Gleichgewichts zwischen relativer Entropie und Redundanz betrachtet wird, ist nicht zu übersehen, dass die Vermehrung der Kombinationsmöglichkeiten nicht das einzige Mittel ist, die überschüssige Redundanz zu verringern. Das gleiche Ergebnis wird erreicht, wenn man die Anzahl der funktionalen Einheiten herabsetzt, der Phoneme oder Quasiphoneme also (s. S. 308, Schema 13). Von einer überschüssigen Redundanz, die ein möglicher späturlappischer dreistufiger quasiphonemischer Quantitätswechsel $(\grave{x} x \sim \grave{x} \sim x$ ) mit sich brachte, konn- 
te sich das Südlappische durch Liquidierung des Stufenwechsels befreien, während in den anderen Dialekten der Schwund der Auslautphoneme des Wortes die Phonemkombinationen vermehrte und somit die Redundanz herabsetzte.

Das Phonologischwerden des Stufenwechsels kann auch durch Untersuchung des wechselnden Informationsgehaltes in den einzelnen Teilen des Wortes überprüft werden. Schon im Frühurlappischen war die Entropie der Phoneme der ersten Silbe und der Stammkonsonanten grösser als die der Phoneme der nichtersten Silben, denn vom Anfang des Wortes bis zum Stammkonsonantismus gab es in allen Stellungen einen reicheren Phonembestand und mehr Kombinationsmöglichkeiten als im Schlussteil des Wortes. In den nichtersten Silben konnten nur vier Vokale $\left({ }^{*} a,{ }^{*} \ddot{a},{ }^{*} n\right.$ und $\left.{ }^{*} \breve{e}\right)$ auftreten und etwas später nur drei $\left({ }^{*} \dot{a}, *_{n}\right.$ und $\left.*^{*}\right)$. Viele der Konsonanten und Konsonantenverbindungen, die an der Grenze zwischen der 1. und 2. Silbe standen, kamen weiter entfernt im Wort nicht in Frage. Durch die Entwicklung eines quasiphonemischen oder phonemischen dreistufigen Quantitätssystems im Stammkonsonantismus wurde der Informationsgehalt des Stammkonsonantismus und somit des ganzen Stammes noch vermehrt. Da sich jedoch der Informationsgehalt des einzelnen Wortes offenbar nicht änderte, bedeutete seine Zunahme in einem Teil des Wortes seine Abnahme im anderen. Der Informationsgehalt der suffixalen Phoneme wurde also noch geringer, Schwund und sonstige Verfallserscheinungen traten noch leichter ein.

Wir haben uns bisher von der Informationstheorie aus lediglich damit beschäftigt, wie der Stufenwechsel phonologisch und dann auch morphologisch zu einem distinktiven Faktor wurde. Das deshalb, weil der Stufenwechsel von allen Wechseln im Innern des Stammes im Lappischen am häufigsten und am meisten morphologisch distinktiv ist. Seine Entwicklungsphasen sind ausserdem klar umrissen und hinreichend bekannt. In gleicher Weise könnten jedoch auch andere Wechsel im Innern des Stammes untersucht werden. Dass z.B. der Metaphoniewechsel im Süd- und im Ostlappi- 
schen phonologisch und in gewissem Umfang auch morphologisch distinktiv wurde, kann als Vermehrung des Informationsgehalts der Silbe mit dem Metaphonievokal auf Kosten des Informationsgehalts der nachfolgenden Silbe angesehen werden. Ein wichtiges Zwischenstadium bildete auch hier die Entfernung der Metaphonievarianten voneinander zu deutlich wahrnehmbaren verschiedenen "Lauten". Da die Metaphonievarianten dabei die Vokalqualität der folgenden Silbe vorausnahmen und vom Standpunkt der Informationsvermittlung somit funktional waren, obgleich sie ihrer Distribution nach Allophone bildeten, waren sie in diesem Stadium typische Quasiphoneme. Durch die Zunahme der funktionalen Symbole in der betonten Silbe des Wortes (und unter bestimmten Bedingungen auch in der unbetonten Silbe) wurde der Informationsgehalt der diesbezüglichen Silbe erhöht und bestimmte redundant gewordene Vokaloppositionen in der nachfolgenden Silbe verschwanden.

\section{DIE NEUTRALISIERUNG DER OPPOSITIONEN}

Ein beachtlicher Teil der morphologisch distinktiven Wechsel im Innern des Stammes betrifft nur die gleichsilbigen Stämme. Hierher gehören mit geringen Ausnahmen u.a. der Stufenwechsel, der durch Überdehnung verursachte Wechsel und der Metaphoniewechsel. Eine Lautveränderung, die den Wechsel distinktiv machte, ein Schwund, eine Kontraktion, eine Verschmelzung o.ä., hat in gewissen Fällen verursacht, dass sich die einzelnen Formen des Wortes äusserlich gleich wurden. So sind z.B. im Norwegisch- und Ostlappischen der Gen. und der Akk.Sg. und im Russischlappischen der Gen., Akk.Sg. und Nom.Pl. nach dem Schwund des auslautenden Konsonanten zusammengefallen, z.B. Gen.,.Akk.Sg. Ip N guole, I kŭèlè, Gen., Akk.Sg., Nom.Pl. lpSk. kualı 'Fisch' $\left(<{ }^{*}\right.$ kolen, ${ }^{*}$ kolem, ${ }^{*}$ kolek $<$ kalan, *kalam, kalat). Es handelt sich meist um Formen mit derart unterschiedlichen syntaktischen Funktionen, dass die Gefahr der Verwechslung nicht besteht. Aus dem..Satzzusammenhang. geht die..jewẹilige. Funktion. der 
Form hervor. Wenn zwei Formen allgemein in gleicher syntaktischer Umgebung auftreten, ist zu beobachten, dass die Auslautkonsonanten ausnahmsweise erhalten sind (s. S. 278282). Der Schwund der Auslautphoneme und die Neutralisierung der Phonemoppositionen der nichtersten Silben beschränkt sich jedoch nicht nur auf im Stamm gleichsilbige Wörter, sondern diese Erscheinung begegnet auch in ungleichsilbigen Wörtern. Da diese Stammtypen nicht in dem Masse Wechsel im Innern des Stammes aufweisen wie die gleichsilbigen Wörter, erfolgt oft ein Zusammenfall der Formen auch in solchen Fällen, wo eine identische syntaktische Umgebung vorliegt. So sind z.B. im Skoltlappischen die Formen der 3. Pl.Prs. und Prät. gleichlautend geworden (s. S. 280). Diese Fälle dürften so zu verstehen sein, dass mit dem Schwund der redundanten Auslautphoneme in dem Stamm nach gleichsilbigen Wörtern und dem Übergang von deren Funktion auf einen Wechsel im Stamminnern der entsprechende Wandel bei den ungleichsilbigen Wörtern mit bedeutend geringerer Frequenz (vgl. a.a.0) analog nach dem Muster der gleichsilbigen Wörter vollzogen wurde. Es gibt direkte Beweise dafür, dass z.B. der Auslautkonsonant zuerst nach der gleichen und später nach der ungleichen Silbe geschwunden ist. So hat Ravila festgestellt, dass sich das ursprüngliche $n$ im Wortauslaut im Westlappischen weithin speziell in dreisilbigen Wörtern erhalten hat ( $M S F O u 62$ S. 92).

\section{DIE VERÄNDERUNG DER LAPPISCHEN MOR- PHOLOGISCHEN METHODE ALS GESAMTERGEBNIS VERSCHIEDENER IMPULSE}

Wir haben oben die Veränderung der lappischen morphologischen Methode als qualitative wie als quantitative Erscheinung behandelt. Dieser Wandel kann nur unter Berücksichtigung aller beteiligter Faktoren verstanden werden. Festgestellt wurde, dass die fusionierende Methode des Lappischen, soweit sie auf der Verwendung von Allomorphen verschiedener .Herkunft. beruht, auf das Urlappische zurückzu- 
führen ist, teilweise vielleicht auch auf ältere Sprachformen. In der Hauptsache besteht die fusionierende Methode jedoch in dem morphophonemischen Wechsel, der in der Schlussphase des Urlappischen und während der Sonderentwicklung der Dialekte entstand. Am reinsten vertritt unter den lappischen Mundarten das Südlappische die fusionierende Sprachform. Es verwendet Suffixe als grammatische Morpheme, verbindet die Morpheme aber äusserst selten mechanisch miteinander. Das Südlappische weicht nicht sonderlich ab vom herrschenden Typus der übrigen finnisch-ugrischen Sprachen. Nur das fast vollständige Fehlen reiner Agglutination macht einen deutlichen Unterschied aus.

In den mittleren und östlichen Dialekten wirkt der Symbolismus neben der fusionierenden Methode. Vom Pitelappischen aus nach Süden nimmt der Anteil des Symbolismus rasch $a b$ und ist im Südlappischen sehr gering. Unbedingte Voraussetzung für die symbolische Methode waren paradigmatische Lautwechsel, die ins Urlappische und teilweise in eine noch ältere Periode zurückreichen. Entscheidend war, dass der frühurfinnische Stufenwechsel von Klusilen, Affrikaten und eventuell auch Sibilanten auf alle Konsonanten und Konsonantenverbindungen ausgedehnt wurde. Dadurch kam es im grössten Teil des Wortschatzes zu einem paradigmatischen Wechsel, der dann einen Teil der Funktionen der Suffixe übernahm. Es ist charakteristisch für den Symbolismus im Lappischen, dass die ursprünglich nur auf bedingten Lautwandel gründenden Wechsel, wie Stufenwechsel, Metaphoniewechsel, Wechsel durch Überdehnung und Strukturtypenwechsel phonologisch und auch Träger einer morphologischen Information wurden, wodurch die Suffixe in der Folge als redundant schwanden oder ihre distinktiven Züge verloren. Eine wichtige Phase in dieser Entwicklung macht der Wandel der Allophone zu Quasiphonemen aus. Man könnte diesen Vorgang als eine Art Neugestaltung der einzelnen Teile des Wortes bezeichnen. Ursprünglich im Schlussteil des Wortes vorhandene distinktive Unterschiede verursachten im Anfang des Wortes einen allophonischen Wechsel. Dieser Wechsel wurde dann als distinktiv betrachtet und die 
ursprünglich distinktiven Unterschiede wurden redundant. Der offene oder geschlossene Charakter der unbetonten Silbe wurde durch die Quantität oder Qualität des vorangehenden Konsonantismus ausgedrückt, der quantitative Sondercharakter bestimmter Kontraktionsvokale und die darin enthaltene Information erschienen in der Überdehnung des vorangehenden Konsonantismus, und die Distinktivität bestimmter Vokalqualitäten der nichtersten Silben wurde zur Distinktivität der Vokalqualität der vorangehenden Silbe. Es handelt sich um eine psychologische Erscheinung, die linguistisch schwer feststellbar ist, für die Diachronie aber beachtliche Bedeutung besitzt. Die auf der Distribution beruhende Phonemanalyse reicht nicht aus, ein Übergangsstadium vom Allophon- zum Phonemwechsel nachzuweisen. Die Distribution der Laute ist entweder komplementär oder nicht. Eine von der Informationstheorie ausgehende Überprüfung dagegen entdeckt eine Zwischenform zwischen Allophon und Phonem, die wir hier Quasiphonem genannt haben. Das Allophon wird zum Quasiphonem, wenn der Sprecher erkennt, das die Wechselvarianten Information über die nachfolgenden Silben enthalten.

Der Wandel der lappischen Morphologie wurde von dem Streben geleitet, zwischen der relativen Entropie und der Redundanz ein bestimmtes ideales Gleichgewicht aufrecht zu erhalten. Als die Redundanz zu stark anzuwachsen drohte, weil die Lautwechsel im Innern des Stammes einen Teil der Funktionen der Suffixe erhielten, folgte als Gegenwirkung der Schwund der Suffixe oder ihrer Teile bzw. ein Ausgleich der in ihnen vorhandenen Oppositionen. Die Tendenz der Aufrechterhaltung dieses Gleichgewichts zwischen relativer Entropie und Redundanz reicht allein jedoch nicht aus, die vor sich gegangenen Veränderungen zu erklären. Wir stellten fest, dass es von vielfältigen und durch die quantitative Informationstheorie nicht erklärbaren Faktoren abhängt, welche suffixalen Elemente schwinden oder ihre Distinktivität verlieren. Wichtig ist das Streben nach regelmässigen Lautveränderungen, "Lautgesetzen", von Bedeutung ist aber auch, welche Funktion das betreffende Suffix ausübt. Das Suffix kann schwinden, auch wenn dadurch zwei identische Formen 
entstehen, wenn nur die syntaktischen Umgebungen der Formen unterschiedlich genug sind. Die Notwendigkeit der Unterscheidung von Formen in gleicher syntaktischer Umgebung trägt wiederum zur Erhaltung der Suffixe bei. Auch semantische Faktoren sind zu berücksichtigen. Obgleich die Dual- und Pluralformen in gleicher Umgebung auftreten, ist doch ihr Unterschied - hauptsächlich durch den Schwund suffixaler Elemente - im Russischlappischen in der possessiven Deklination und Konjugation völlig aufgehoben mit Ausnahme des Dialekts von Paatsjoki sowie teilweise auch in den anderen lappischen Mundarten. Die Ursache wird wohl darin liegen, dass der semantische Unterschied zwischen Dual und Plural nicht besonders bedeutsam ist. Ein semantisch wichtiger Unterschied wie z.B. der zwischen 1. und 2. P.Sg. hat sich dagegen in allen Dialekten gut erhalten, und zwar ungeachtet dessen, dass die suffixalen Elemente, wodurch die Formen getrennt werden ( $-m$ und $-k$ ), lautlich gesehen sehr leicht schwanden. Die analogen Einwirkungen zwischen verschiedenen Formen und Formgruppen waren ebenfalls von Bedeutung für die morphologische Entwicklung des Lappischen. Phonetisch gesehen ist es klar, dass in einer Sprache, deren erste Silbe stets hauptbetont ist, deren letzte Silbe völlig unbetont und wo die übrigen Silben entweder nebenbetont oder unbetont sind, der Auslaut leicht verfällt, reduziert wird oder sogar schwindet. Die Entwicklung der im Auslaut stehenden Laute kann ferner durch Sandhiwechsel an der Wortgrenze entscheidend beeinflusst werden. Alle diese Impulse wurden jedoch sichtlich die ganze Zeit von der Tendenz der Aufrechterhaltung des Gleichgewichts zwischen relativer Entropie und Redundanz reguliert wie von einem statistischen Mechanismus.

\section{DIE ALLGEMEINGÜLTIGKEIT DER ERZIELTEN ERGEBNISSE - EINIGE PARALLELEN}

Haben die in der morphologischen Entwicklung des Lap-

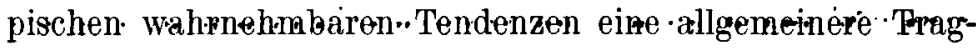


weite oder erweist sich die Kausalität in der Entwicklungsreihe als scheinbar und auf blossem Zufall beruhend? Die Frage kann erst dann beantwortet werden, wenn entsprechende Veränderungen in genügend vielen Sprachen untersucht worden sind. Was speziell den Anteil der konstanten Redundanz als Veränderungen regulierenden Faktor angeht, so darf nicht vergessen werden, dass wirklich eine grosse Veränderung in der Anzahl der Phoneme oder der Kombinationsmöglichkeiten nötig ist, bevor die Redundanz bedeutend steigt oder sinkt. Die Entstehung eines neuen Phonems in einer Sprache z.B., die bereits 32 Phoneme besitzt und deren Redundanz $70 \%$ beträgt, führt nur zu einer Erhöhung der Redundanz auf $70,26 \%$, wenn angenommen wird, dass die wirkliche Entropie unverändert bleibt. So geringe Redundanzveränderungen bedeuten wohl zunächst noch gar nichts. Die quantitativen Verfahren der Informationstheorie helfen also bei der Erforschung kleiner Veränderungen im Phonemsystem und struktureller Details nicht weiter. Falls sich in der Zukunft Verfahren entwickeln lassen, nach denen die Redundanz der Sprachen genauer als heute berechnet werden kann, werden wir auch genauere Kenntnisse erhalten über den Wechselbereich der Redundanz und die auf den Wechsel einwirkenden Faktoren. Dann könnten die Möglichkeiten der Informationstheorie vielleicht auch effektiver auf die diachronische Linguistik angewendet werden. Wir wollen hier nicht das auf der Informationstheorie beruhende Modell anwenden auf typologische Veränderungen in anderen Sprachen, sondern lediglich überblickartig einige in den ostseefinnischen und germanischen Sprachen eingetretene Veränderungen behandeln, in denen vielleicht Parallelen zur Entwicklung im Lappischen zu sehen sind.

Ein morphophonemischer Wechsel, wie er einer reinen Agglutinationssprache fremd ist, begegnet in mässigem Ausmass in den finnisch-ugrischen Sprachen, wie die Agglutinationsindexe (Tabelle 2, S. 221) zeigen. Einen Überblick über die in den einzelnen uralischen Sprachen im Stamm erscheinenden Wechsel gibt Valter Tauli in seiner Arbeit "Structural Tendencies in Uralic Languages» (The Hague) S. 183-195. 
Die meisten finnisch-ugrischen Sprachen haben eher als agglutinierend-fusionierend denn als agglutinierend zu gelten. Wenn alle an der Morphemgrenze erscheinenden Wechsel (z.B. fi. Ad.Sg. kive-llä - Ad. Pl. kiv-i-llä 'Stein', 1. Sg.Prs. vedä-n - 1. Sg.Prät. ved-i-n 'tragen', kala - Part.Sg. kal-ā 'Fisch') als zur fusionierenden Yethode gehörend angesehen werden und nur die wirklich im Innern des Stammes erscheinenden Wechsel berücksichtigt werden (z.B. fi. dial. Nom.Sg. + Px 2. Sg. akkas - In.Sg. akas 'Weib', liv. Nom.Sg. täm Gen.Sg. tam 'Eiche'), ist die morphologische Funktion von Wechseln im Stamminnern ausserhalb des Lappischen relativ selten. Ein systematischer Symbolismus, der umfangreiche Formgruppen betrifft, begegnet neben dem Lappischen nur im Estnischen und Livischen. In allen anderen finnisch-ugrischen Sprachen gibt es nur zufälligen Symbolismus.

Im Estnischen ist die Quantität in Fällen folgender Art morphologisch distinktiv:

Gen.Sg. - Part.Sg. (Ill.Sg): vă̌kka - val̀ka 'Scheffel', lin̆na - liǹna 'Stadt', kōli-kôli 'Schule', ilma - ilma 'Luft, Wetter', pēsasti ̌̌ku - pēsastikkku 'Gebüsch';

Nom.Sg - Gen.Sg.: ĭkke - ik̀ke 'Joch', hōne - hône 'Gebäude';

Nom.Sg. - In.Sg.: rat̀tas - rattas 'Rad', pẹsas - pệsas 'Strauch';

Part.Sg. - El.Sg.: raìtast-raìtast, tōrest-tôrest 'roh';

Nom.Sg. - Ill.Sg.: tuвa - tup̀pa 'Stube';

Gen.Sg. - Ill.Sg.: lume - lumme 'Schnee';

Part.Sg. - Ill.Sg.: jęge - jèkke 'Fluss';

2. Sg.Imp. - Inf.: jätta - jätta 'verlassen'.

(S. Lauri Posti JSFOu 54,2 S. 10-12; Hildegard Must FUF 33 S. 155-157.)

Im Livischen ist neben der Quantität oft auch der Vokal der zweiten Silbe distinktiv, z.B. (Kettunen, Livisches Wörterbuch mit grammatischer Einleitung; Postj MSFOu 85)

Nom.Sg. - Part.Sg. (Ill.): kalà - kăllâ 'Fisch', jālga-

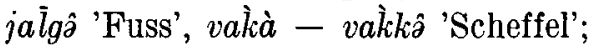

Ad.Sg. — All.Sg.: jälgal — ja lgâl;

Nom.Sg. - Ill.Sg.: lāmbaz - lā̄bâz 'Schaf'; 
Part.Sg. - Nom., Gen.Pl.: ibist - ibbist 'Pferd', pi̋̌kist pi卂kist 'Donner'.

Im Estnischen gibt es einen Wechsel zwischen langer und gedehnter Quantität, wenn die erste Silbe lang ist. Wenn im Stammkonsonantismus eine Geminate oder eine Konsonantenverbindung steht, unterliegt diese dem Wechsel, handelt es sich aber um einen Einzelkonsonanten, berührt der Wechsel nur den langen Vokal und den Diphthong der ersten Silbe (s. z.B. Tauli $J S F O u ~ 57,6$ S. 1-2). Paradigmatischer Wechsel zwischen kurzer und gedehnter Quantität erscheint, wenn die erste Silbe ursprünglich kurz war. Der Stufenwechsel der Klusile ist ein morphologisch distinktiver Faktor, betrifft aber nur einen relativ geringen Teil des Wortschatzes, z.B. Nom.Sg. tuba - Gen.Sg. toa 'Stube' usw.

Im Livischen begegnet immer dann ein Wechsel, wenn in der ersten Silbe der Vertreter eines ursprünglich kurzen Vokals oder eines Diphthongs steht, dann aber nicht, wenn die erste Silbe den Vertreter eines ursprünglich langen Vokals enthält (Posti MSFOu 85 S. 296-300).

Vor allem das estnische Quantitätssystem hat ständig Anlass gegeben zu reger wissenschaftlicher Diskussion in synchronischer wie auch in diachronischer Sicht (s. Posti JSFOu 54,2, CSIFU I S. 408-418; Collinder Studia Linguistica 5; Mägiste, Meddelanden från seminarierna för slaviska språk, jämförande språkforskning och finsk-ugriska språk vid Lunds Universitet, Nr. 1, Lund 1951, S. 5-12; Raun Studia Linguistica 8; Tauli JSFOu 57,6; Hildegard Must FUF $33 \mathrm{~S}$. 146-163; Ilse Lehiste, American Studies in Uralic Linguistics, Bloomington 1960, S. 21-82, Liiv Keel ja Kirjandus 1961 S. 412-424; Ravila Vir. 1961 S. 345-350; Harms, Estonian Grammar, The Hague 1962, S. 143-162; Hallap, Nonaginta, Tallinn 1963, S. 95-122; mit Hinweisen jeweils auf das frühere Schrifttum). Für die gedehnte und die lange Quantität sind verschiedene sprachgeschichtliche Erklärungen abgegeben worden. Es lässt sich leicht feststellen, dass die gedehnte Quantität vor der ursprünglich offenen Silbe steht und die lange Quantität vor der ursprünglich geschlossenen

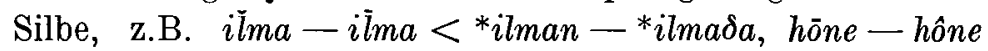


$<{ }^{*}$ hōneh — *hōnehen, pèsas - pềsas < * pensas - *pen-

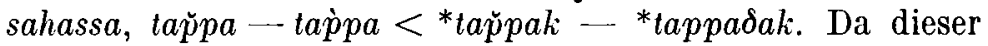
Wechsel stark an den Stufenwechsel der Geminataklusile im Ostseefinnischen erinnert, ist es nicht verwunderlich, dass man ihn auch auf dieser Basis hat erklären wollen. E. N. Setälä vertrat die Ansicht, der estnische Quantitätswechsel sei die direkte Fortsetzung des uralten Stufenwechsels, der alle Konsonanten betraf (JSFOu 14,3 S. 8-). Auch Tauli führte den estnischen Quantitätswechsel teilweise auf den Stufenwechsel zurück. Dazu wurde er durch die phonetischen Messungen von J. Laurosela veranlasst, wonach auch in den finnischen Dialekten von Südostbottnien der Konsonantismus an der Grenze zwischen der ersten und zweiten Silbe vor der geschlossenen Silbe kürzer ist als vor der offenen. Nach Tauli ist die Kürzungstendenz der Konsonantenverbindungen und Geminata vor der geschlossenen Silbe älter als das heutige Quantitätssystem des Estnischen. S. JSFOu 57,6 S. 6-10. Man dürfte heute verbreitet darin übereinstimmen, dass der estnische Quantitätswechsel auf früheren Betonungsunterschieden beruht. Zuletzt hat Lauri Posti auf den entscheidenden Charakter der ursprünglich offenen oder geschlossenen Beschaffenheit der zweiten Silbe hingewiesen: "Im Estnischen wiederum gehen die Quantitätsstrukturunterschiede in den Typen ${ }^{2}$ [lang] und ${ }^{3}$ [überlang] auf jenen Betonungsunterschied zurück, der meiner Meinung nach zwischen einer geschlossenen und einer offenen zweiten Silbe entstand." (CSIFU I S. 418, Vgl. auch Posti MSFOu 85 S. 301-, JSFOu 54,2 S. 13-14; Ravila Vir. 1936 S. 208-214). Durch Unterschiede in der Betonung wurde offenbar auch die Greminierung eines ursprünglichen Einzelkonsonanten nach einem kurzen Vokal der ersten Silbe vor einem Kontraktionsvokal verursacht (

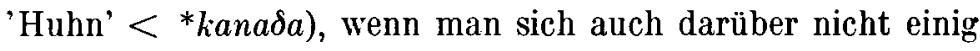
war, ob die Erscheinung als stellvertretende Dehnung durch den Silben- oder Vokalschwund zu werten ist (M. Weske, Untersuchungen zur vergleichenden Grammatik des finnischen Sprachstammes, Leipzig 1873, S. 13; Collinder, Über den finnischlappischen Quantitätswechsel, Uppsala 1929, S. 1-, 21-, 
Uppsala universitets airsskrift 1939: 1 S. 67; Posti MSFOu 85 S. 304) oder auf den langen Vokal der zweiten Silbe zurückzuführen ist (V. Porkka, Über den ingrischen Dialekt, Helsingfors 1885 , S. $40--$; K. B. Wiklund MO 9 S. 229-; Kettunen, Suomen murteet II, Helsinki 1930, S. 183; Ariste, Hiiu murrete häälikud, Tartu 1939, S. 148; E. Itkonen, Kieli ja sen tutkimus, Helsinki 1966, S. 177-178).

Auf jeden Fall wird man davon ausgehen können, dass der Quantitätswechsel phonetisch bereits vorhanden war, bevor die Apokope und auch der Schwund bestimmter Auslautkonsonanten sowie die Kürzung der langen Vokale in den nichtersten Silben zu einem Wegfall der Suffixe geführt hatten. In seinem in finnischer Sprache geschriebenen Artikel »Ist das Estnische seiner Betonung nach eine stark zentralisierte Sprachform?" (Vir. 1936) schreibt Paavo Ravila: "Denken wir an solche Bildungen wie $m u \bar{s} t<{ }^{*} m u s t a, m u \bar{s} t a<{ }^{*} m u s-$ $t a \delta a$, so ist festzustellen, dass es deutlich zentralisierte Worterscheinungen sind. Die erste Silbe hat im Vergleich zur sonstigen Umgebung die herrschende Stellung inne, sie bildet eindeutig das Zentrum. Dieses Zentrum wurde dann noch effektiviert, was psychologisch sehr gut verständlich ist, und die Entwicklung führte im ersten Fall zu einem Schwund des Vokals in der unbetonten Silbe und im zweiten Beispiel zu einer Kürzung des aufgrund des Spirantenschwundes entstandenen langen Vokals." (S. 212) Zweifelsohne muss die chronologische Beziehung zwischen der Entwicklung des Quantitätssystems und dem Verfall des Vokalbestands der nichtersten Silben gerade auf diese Art und Weise verstanden werden.

Obgleich der Quantitätswechsel im Livischen in mancher Hinsicht vom estnischen Quantitätswechsel abweicht, muss doch auch für das Livische ein phonetischer Quantitätswechsel angenommen werden, bevor es in den Oppositionen der nichtersten Silben zu einer Neutralisierung kam, die dann zum heutigen System führte. Die Reihenfolge war dann offenbar folgende: kala - ${ }^{*} k a l a \delta a>k a l a-k a l a ̈>k a l a-$

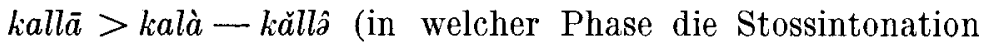
auftrat, ist in diesem Zusammenhang nicht wesentlich). In 
Fällen wie Part.Sg. - Nom., Gen.Pl. ibist - ibbist, piťkist piťkist beruht die Verlängerung im Nom. und Gen.Pl. auf dem Muster des Nom.Sg. (ibbi, pit̄ki) (Posti MSFOu 85 S. 311-312), und sie kann wohl nicht als Voraussetzung für das Zusammenfallen des Suffixteils vom Part.Sg. und Nom.Pl. angesehen werden.

Es erhebt sich die Frage, ob im Estnischen der Quantitätswechsel in gleicher Weise distinktiv wurde wie im Lappischen, d.h. ob die in den Quantitätsstufen enthaltene Information hinsichtlich der letzten Silben des Wortes eine Zunahme der Redundanz in den nichtersten Silben verursacht und damit den Verfall der Suffixe gefördert hat. Ein Vergleich mit den anderen ostseefinnischen Sprachen scheint in vieler Hinsicht darauf hinzuweisen, dass die Frage positiv zu beantworten ist. Im Estnischen und Livischen, wo der Quantitätswechsel im Innern des Stammes am weitesten entwickelt ist, sind auch die Suffixe am stärksten verfallen. Bei den Stammtypen, die keinen Wechsel im Stamminnern kennen, haben die Suffixe ihre Distinktivität am besten bewahrt. Teilweise beruht das zwar schon auf urfinnischer Prädisposition. So erklärt sich z.B. die erhaltene Partitivendung im Typus raamatut $\left(<{ }^{*}\right.$ rāmattuta) 'Buch' und die geschwundene Endung im

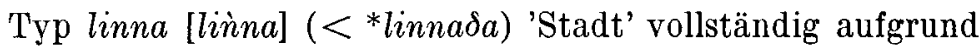
urfinnischen suffixalen Stufenwechsels. Beachtenswert ist jedoch, dass sich im Estnischen andererseits nicht die suffixale schwache Stufe so verallgemeinert hat wie z.B. im Finnischen (fi. raamattua wie laulua $<*^{*}$ lauluda pro*raamattuta). Eine derartige Analogie hätte im Estnischen zum Zusammenfall von Partitiv und Genitiv geführt (vgl. Gen.Sg. raamatu). Natürlich kann man nicht behaupten, dass sich im Estnischen die schwachstufige Variante durchgesetzt hätte, falls der Unterschied zwischen Genitiv und Partitiv irgendwie erhalten geblieben wäre. Eine Erscheinung aber, die deutlich auf die Aufrechterhaltung der Opposition zielt, ist die Verbreitung der Illativendung -sse in Stämmen, die nicht vom Quantitätswechsel erfasst werden, z.B. raamatusse, vgl. lumme $<$ lumehen (daneben auch anal. lumesse) 'in den Schnee'. Ursprünglich begegnete - sse $<-$ sehen $\left(<{ }^{*}-\right.$ sen $)$ nur nach einer auf $h$ 
anlautenden Silbe, z.B. peresse $\left(<{ }^{*}\right.$ perehesehen $<{ }^{*}$ perehesen $)$, -sehen pro -sen anal. zum Typus punaise-hen (s. Setälä, Yhteissuomalainen äännehistoria, Helsinki 1890-91, S. 248-249). Eine bedeutendere Neutralisierung der Oppositionen tritt nur in solchen zweisilbigen vokalstämmigen Nomina auf, deren erste Silbe kurz ist. Der Nom., Gen. und Part. Sg. dieser Nomina sind zusammengefallen, z.B. kala < kala, $-n$, ${ }^{*}-\delta a$. Auch in diesem Typus hat sich der Partitiv dialektweise von Nom. und Gen.Sg. getrennt erhalten, ohne dabei mit dem Illativ zusammenzufallen, z.B. estnS Nom., Gen.Sg. kala, Part. ka lla, Ill. kalla.

Ausserhalb des Estnischen und Livischen gibt es im Ostseefinnischen keinen ausgedehnten Wechsel im Innern des Stammes. Der Stufenwechsel betrifft nur die Klusile, und auch die verschiedenerseits vorhandene Gemination, deren Voraussetzungen und Einflussbereich dialektweise wechseln, verursacht keinen so allgemeinen systematischen paradigmatischen Wechsel, dass er als morphologisch distinktiver Faktor bedeutsam werden könnte. So haben denn auch die Suffixe ihre Distinktivität gut erhalten, ausser im Estnischen und Livischen. Wichtig ist die Feststellung, dass es z.B. im Wepsischen trotz zahlreicher wortkürzender Lautwandel wie Kürzung der langen Vokale, Syn- und Apokope, nicht zu einem signifikanten morphologischen Synkretismus kam, sondern die Oppositionen haben sich speziell in den Suffixen erhalten.

Die einzige ostseefinnische Sprachform, wo die Suffixe sichtbar ihre Distinktivität eingebüsst haben und doch kein entsprechender Wechsel im Innern des Stammes begegnet, sind die südwestfinnischen Dialekte. Der Schwund von auslautendem $t$ und $n$ und die Kürzung von langen Vokalen und Diphthongen in nichtersten Silben haben bewirkt, dass z.B. bei vielen, in der Sprache allgemeinen Nominaltypen der Nom., Part.Sg. und der Nom.Pl. zusammengefallen sind, z.B. kalà < kala, kalan, kalaa, kalat 'Fisch'. Die einen Quantitätswechsel bewirkende Gemination betrifft nur einen Klusil oder Sibilanten vor einer Silbe mit einem Kontraktionsvokal und nach einem Vokal oder stimmhaften Konsonanten, z.B. Nom.Sg. sotà-Part. sotta 'Krieg'. Auch unter Berücksichtigung 
dessen, dass das $t$ im Wortauslaut nicht geschwunden ist, wenn das folgende Wort mit einem Klusil oder Sibilanten begann und dass das $n$ im Wortauslaut durch einen Nasal vertreten ist, falls das nächste Wort mit einem Klusil beginnt (silmäp pääs 'Augen im Kopf', kalàm pää 'Kopf des Fisches'), bleiben doch noch eine Reihe von Fällen, wo das Wort allein nicht auszudrücken vermag, zu welcher morphologischen Kategorie es gehört. Wir haben hier nicht die Möglichkeit zu klären, in welcher Weise in den finnischen Südwestdialekten die Neutralisierung der morphologischen Oppositionen kompensiert worden ist und welchen Anteil z.B. syntaktische und satzprosodische Faktoren an der Kompensation haben. Hingewiesen werden kann auf Erscheinungen wie die, dass in den Südwestdialekten in der 3. Pl.Prs. der Verben allgemein die ursprüngliche Form mit der Endung $v a(t)$, vä( $(t)$ verwendet wird, während in den meisten anderen finnischen Dialekten anstelle der 3. Pl. die Form der 3. Sg. steht. So unterscheidet sich der Plural vom Singular, auch wenn der Numerusunterschied nicht im Subjekt zum Ausdruck kommt. Ferner ist in einigen Fällen an die Stelle eines geschwundenen Suffixes analog ein anderes aus Paradigmen getreten, wo sich das Suffix erhalten hat, z.B. Ill.Sg. talòsse, Ill.Pl. talòssi (vgl. estn.), 3. Sg.Prät. istus, sanòs (die den schriftspr. und urspr. Typen istui, sanoi entsprechenden Formen sähen in den Südwestdialekten aus wie die Präsensformen istu, sanò; das s stammt aus den Kontraktionsverben).

Im Livischen sind die durch Metaphonie und andere regressive Wirkungen des Vokals der zweiten Silbe verursachten Wechsel in gewissem Umfang morphologisch distinktiv geworden, z.B. (Posti) Nom.Sg. tǟ --Gen.Sg. ta $\bar{m}$ 'Eiche', läpš-laps 'Kind', jǒia-jŏug 'Fluss', kēl-kiel 'Zunge, Sprache'. Auch die Palatalisierung des Stammkonsonantismus kann morphologisch distinktiv sein, z.B. Nom.Sg. nuŕm

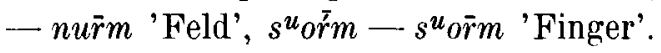

Wenn in der zweiten Silbe ein ursprüngliches $i$ oder $j$ stand und an der Grenze zwischen erster und zweiter Silbe ein $m$, $v, p, b, k$ oder $g$, wird in der ersten Silbe $a>\ddot{a}, o>\ddot{o}(>e)$, 
$u>\ddot{u}(>i)$. Hierdurch ergab sich ein paradigmatischer Metaphoniewechsel in ursprünglichen zweisilbigen $e$-stämmigen Nomina, in deren zweiter Silbe am Ende des Wortes $e$ zu $i$ wurde. Da im Livischen das $n$ im Wortauslaut geschwunden ist und ausserdem Apokope eintrat, die auch den vor dem geschwundenen $n$ stehenden Vokal betraf, kam es zu solchen Formpaaren wie die Beispiele im vorangehenden Absatz zeigen $(\operatorname{tä} \bar{m}-t a \bar{m}<$ tammi - tammen). Wenn an der Grenze zwischen der ersten und zweiten Silbe ein Dentalkonsonant oder eine einen Dental enthaltende Konsonantenverbindung steht, gibt es keine Metaphonie, sondern der Dental ist durch die Einwirkung des folgenden $i$ oder $j$ mouilliert. Hierdurch kam es zur morphologischen Distinktivität der Palatalkorre-

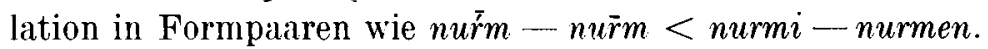
Zur Metaphonie sind auch die Veränderungen $\ddot{a}>e, \bar{a}>\bar{e}$ in der ersten Silbe zu rechnen, die vor einem $i$ und $j$ der zweiten Silbe unabhängig davon eingetreten sind, wie der Stammkonsonantismus aussieht, z.B. kèg $g_{G}$ 'Kuckuck', sērr, sēr 'Schienbein'. Von den Vokalwechseln in der ersten Silbe verursacht durch den Wechsel $i \sim e$ der zweiten Silbe - ist noch das erhaltene $\bar{e}$ und $\bar{o}$ vor einem $i$ und $j$ der zweiten Silbe zu erwähnen, sonst entwickelte sich $\bar{e}>i e$ und $\bar{o}>\ddot{u} 0$. Morphologisch distinktiv ist die erstgenannte Erscheinung im Typus $k \bar{e} l-k i e l<{ }^{*} k e \bar{e} l i-{ }^{*} k e \bar{l} l e n$. Unter bestimmten Bedingungen kam es anstelle von Metaphonie zu Epenthese. Neben der sog. $i$-Epenthese begegnet auch die $u$-Epenthese. Durch Epenthese wurde z.B. in dem Fall jǒia - jǒug < $<{ }^{*}$ jogi*jogun $<$ joki-*joyen ein morphologisch distinktiver Wechsel bewirkt.

Nach Lauri Posti hat man sich die Entstehung von Metaphonie, Epenthese und Mouillierung im Livischen so zu denken, dass ein $i$ oder $j$ in der zweiten Silbe den vorangehenden Konsonanten palatalisierte, die Labial- und Palatalkonsonanten aber später ihren palatalen Charakter verloren haben, der auf die vorangehende Silbe überging und eine Metaphonie oder Epenthese verursachte ( $M S F O u$ 85 S. 106). In einer bestimmten Entwicklungsphase des Livischen begegneten in den nichtersten Silben nur die Vokale $a, u$ und $i$, die den 
vorangehenden Konsonantismus dahingehend beeinflussten, dass er vor $a$ velarisiert, vor $u$ labiovelarisiert und vor $i$ palatalisiert wurde. Diese verschiedenen Konsonantismen hatten wieder ihre eigene Wirkung auf den vorangehenden Vokalismus, wo es unter bestimmten Bedingungen zu Umlaut, Brechung, $i$-Epenthese oder $u$-Epenthese kam (Posti a.a.0. 289 -290, Uppsala universitets årsskrift 1948 S. 39-59). Eine andere Auffassung über die Metaphonie im Livischen und auch in den germanischen Sprachen hat Björn Collinder vertreten. Er meint, die Metaphonie sei ursprünglich ohne Vermittlung des Konsonantismus geschehen und nur wenn der die Metaphonie verursachende Vokal der zweiten Silbe geschwunden sei (Uppsala universitets årsskrift 1939: 1 S. 71-75). Bo Wickman hat sich der Auffassung von Collinder angeschlossen (Uppsala universitets àrsskrift 1960 S. 32-48).

Ob im Umlaut eine Kontaktassimilation durch Vermittlung des Konsonantismus zu sehen sei oder eine antizipationsähnliche Fernassimilation, ist in der Lautgeschichte der germanischen Sprachen lange diskutiert worden. Zahlreiche Germanisten sind eingetreten für die auf der Annahme der Kontaktassimilation fussende "Mouillierungstheorie» oder "Kontaktwirkungstheorie» (s. z.B. Franck, Altfränkische Grammatik, Göttingen 1909, § 13; Luick, Historische Grammatik der englischen Sprache, Leipzig 1914, §200; Rooth, Lunds universitets årsskrift N.F. Avd. 1, Bd. 25, No.6, 1929, S. 109-151, Vetenskaps-Societeten $i$ Lund, Arsbok 5, 1935, S. 15-, Studia Neophilologica 13 S. 103-; Kranzmayer, Zeitschrift für Mundartforschung 14, 1937, S. 73-100; Streitberg, Urgermanische Grammatik, Heidelberg 1943, S. 79). Jakobi und Steinthal stellten schon Mitte des vorigen Jahrhunderts die Theorie auf, die Metaphonie in den germanischen Sprachen habe sich ohne Vermittlung des Konsonantismus vollzogen (Jakobi, Beiträge zur deutschen Grammatik, Berlin 1843, S. 25; Steinthal Zeitschrift für Völkerpsychologie und Sprachwissenschaft 1860:1 S. 113-116); auch später versuchte man diese Theorie mal mit psychologischen, mal mit phonetischen Kriterien zu untermauern. Die vielen schwerwiegenden Argumente, die die Forschung der letzten Jahrzehnte gegen die Theorie der Kon- 
taktassimilation vorgebracht hat, scheinen der Antizipationstheorie zugute gekommen zu sein und immer mehr Wissenschaftler davon überzeugt zu haben, dass die Metaphonie in erster Linie den Vokalismus des Wortes betraf und relativ unabhängig war von dem zwischen den Vokalen stehenden Konsonantismus (z.B. Sommerfelt, Festskrift til Hjalmar Falk, Oslo 1927, S. 42-, Norsk Tidskrift for Sprogvidenskap 7, 1934, S. 355-362; Brinkman, Sprachwandel und Sprachbewegungen in althochdeutscher Zeit, Jena 1931, S. 78; Twaddel, Monatshefte für den deutschen Unterricht 30, 1938, S. 177181; Hesselman, Omljud och brytning i de nordiska språken, Uppsala 1945, S. 6-; Penzl, Language 25, 1949, S. 233-236; Martinet, Économie des changements phonétiques, Berne 1955, S. 201; Szulc, Umlaut und Brechung, Poznań 1964). Ferner ist die Auffassung geäussert worden, dass es sich bei der Frage nach der Entstehungsart der Metaphonie um ein Scheinproblem handle, da z.B. beim $i$-Umlaut der Wandel der Formantenstruktur automatisch sowohl den Vokal der Silbe vor dem $i$ als auch den dazwischen stehenden Konsonantismus einbeziehe (Malmberg, Nya vägar inom språkforskningen, Stockholm 1959, S. 141). Erwidert wurde hierauf, eine leichte Angleichung des Konsonantismus an die palatale Umgebung sei doch etwas ganz anderes als eine eigentliche Mouillierung (Wickman, Uppsala universitets årsskrift 1960 S. $32-48$ ).

Bei der Erörterung der Entstehung von Metaphonie, regressiver Mouillierung und Epenthese im Livischen dürfte es nützlich sein, kurz den Charakter der regressiven Mouillierung in einigen anderen ostseefinnischen Sprachen zu überdenken. Das Problem ist kürzlich von Terho Itkonen behandelt worden (MSFOu 145 S. 76-103). In den Ostdialekten des Finnischen liegt die regressive Mouillierung in solchen Fällen wie $t u l^{\prime}$ 'Feuer', suutaŕ 'Schuster', kottiin 'nach Hause' an der Grenze zwischen Vokal und Konsonant. Nach Itkonen "kann der Vokal von Anfang an leicht heller sein, wird seinem Ende zu immer $i$-artiger und gibt schliesslich dem Beginn des folgenden Konsonanten eine $i$-Färbung, die schon dem Gehör nach mit dem Fortschreiten des Konsonanten abnimmt (bei Geminaten in dem 
Ausmass, dass der letzte Teil des Konsonanten ziemlich deutlich nichtmouilliert ist)" (a.a.O. 78). Hinsichtlich der Beziehung zwischen progressiver und regressiver Mouillierung kommt Itkonen zu der Folgerung, die im Altkarelischen entstandene regressive Mouillierung habe sich ursprünglich speziell auf den Übergang vom Vokal zum Konsonanten konzentriert. Die Stelle des ostfinnischen Palatalisationsübergangs — von früheren Forschern bereits geschildert - ist nun auch experimentellphonetisch bestätigt worden (Sovijärvi, Verba docent, Helsinki 1959, S. 440). Die regressive Mouillierung im Estnischen ist ebenfalls ihrem Charakter nach ein Palatalisationsübergang zwischen Vokal und Konsonant (s. Liiv Cosemcroe финно-угроведение 1965, S. 33-36). Im Dialekt von Tartu (Dorpat) begegnet im Konsonanten keine Mouillierung, sie ist ganz auf das Ende des Vokals konzentriert, wo ein kurzes $i$ hörbar wird (Hella Keem, Emakeele Seltsi Aastaraamat 4 S. 145-151). Nach Terho Itkonen erklärt sich der sog. "Epenthesetypus" poikain 'mein Sohn, meine Söhne', poikais 'dein Sohn, deine Söhne', makkais 'lag' in den finnischen Südsavound Südostdialekten als Entwicklung aus einem regressiven Palatalisationsübergang ( $M S F O u 145$ S. 87).

Wie auf den S. 239-246 festgestellt wurde, kann die lappische Metaphonie nicht überzeugend als Resultat einer Kontaktassimilation dargestellt werden, sondern es handelt sich eher um eine antizipationsähnliche Fernassimilation. Die im Zusammenhang mit der Palatalmetaphonie im Süd- und Ostlappischen auftretende Mouillierung oder Koronalisation erinnert stark an den ostfinnischen Palatalisationsübergang (s. S. 242-245). Es ist m.E. nicht unmöglich, auch die livische Metaphonie, Epenthese und regressive Mouillierung als Produkte einer Fernassimilation zu erklären, mit dem Ausgangspunkt des Palatalisationsübergangs. Dann erklärt sich die sog. "i-Epenthese" leicht als Verstärkung des Palatalisationsübergangs speziell im Schlussteil des Vokals, wie es im Dialekt von Tartu (Dorpat) und in einigen Mundarten von Südostfinnland der Fall war. Es fehlt dann nur noch ein kleiner Schritt und die Palatalität erstreckt sich auf den ganzen Vokal der ersten Silbe, was gleichbedeutend ist mit Meta- 
phonie. Es ist vollauf verständlich, dass sich der Palatalisationsübergang speziell vor labialen und palatovelaren Konsonanten zu "Epenthese» und Metaphonie entwickelt hat. In der Richtung dieser, i.a. einer Mouillierung widerstehenden Konsonanten konnte sich der Palatalisationsübergang nicht so leicht ausbreiten wie nach rückwärts, in Richtung des Vokals. Wenn aber vor $i$ und $j$ ein der Mouillierung geneigter Dentalkonsonant stand, verschob sich der ursprünglich an der Grenze zwischen dem Vokal der vorangehenden Silbe und dem Dental liegende Palatalisationsübergang natürlich nach vorn, in den Konsonantismus. Für diese Annahme spricht die Tatsache, dass die zweite Komponente der auf $i$ endenden Diphthonge der betonten Silbe in gleicher Weise ihre Palatalität an den folgenden Dental verloren hat, so dass der Diphthong heute durch einen langen Vokal vertreten wird, während der folgende Dentalkonsonant mouilliert ist, z.B. kānal (daneben kàinal) 'Achselhöhle', ùška ( livW ùiska) 'Schlange', pừsi (< puissa) 'in den Bäumen' (Posti MSFOu 85 S. 285). Es gibt auch andere Belege, die daranf hinweisen, dass auf $i$ endende Diphthonge in irgendeiner Phase teilweise zusammenfallen konnten mit den von uns angenommenen Fällen Vokal + Palatalisationsübergang. So ist in der unbetonten Silbe die Entwicklung $a i>\ddot{a}$ bekannt, z.B. livW Part.Sg. sìemnăggâ (Nom.Sg. siemnàiga 'Essen, Mahlzeit, Speise', a.a.O. 30), die einer metaphonischen Entwicklung $a+$ Palatalisationsübergang $>\ddot{a}\left({ }^{*} t a^{i} \bar{m}>t \ddot{a} \bar{m}\right)$ völlig entsprechen würde. Um einen systematischen Zusammenfall kann es sich nicht handeln, doch scheinen auch diese Erscheinungen unsere Annahme von der Herkunft der regressiven Palatalisationsassimilationen im Livischen $\mathrm{zu}$ stützen. In gleicher Weise kann die sog. $u$-Epenthese aufgefasst werden als Vorartikulation des $u$ der zweiten Silbe, ohne dass der Konsonantismus an der Vermittlung der Labialität teilgenommen hätte. Weiterhin ist es durchaus möglich, dass auch die Brechung im Livischen als Fernassimilation durch den Einfluss des $a$ und $u$ der zweiten Silbe und nicht durch Vermittlung des Konsonantismus geschehen ist, z.B. $l^{i} e \grave{p} a ̀<$

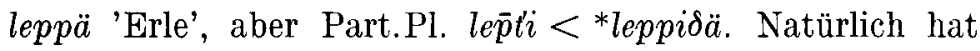


sowohl beim Umlaut und bei der Epenthese als auch bei der Brechung auch der dazwischenstehende Konsonantismus einen Anstrich von Palatalität resp. Velarität erhalten, doch braucht nicht unbedingt angenommen $\mathrm{zu}$ werden, dass die Veränderung zuerst im Konsonantismus eingetreten und dann erst auf den vorangehenden Vokalismus übergegangen wäre. Ich hoffe in anderem Zusammenhang zurückzukommen auf die phonetischen und phonologischen Details dieser Frage.

Wichtiger für unser Thema ist, ob es im Livischen ursprünglich nur dann zur Metaphonie kam, wenn der sie hervorrufende Laut schwand, oder ob die Erhaltung bzw. der Schwund des diesbezüglichen Lautes keinen Einfluss darauf hatte. Meines Erachtens liegt keinerlei Notwendigkeit vor, wie Collinder anzunehmen, dass die Metaphonie nur beim Schwund des $i$ oder $j$ der nächstfolgenden Silbe möglich sei. Auch Wickman vermochte kein einziges schlagendes Argument zur Stützung der Hypothese Collinders vorzubringen. Die in verschiedene Richtungen gehenden Analogien haben die ursprünglichen Verhältnisse in dem Masse verwirrt, dass es schwer ist, die Frage mit unbedingter Sicherheit so oder so zu entscheiden. Von welcher Hypothese wir auch ausgehen, stets lässt sich ein grosser Teil der abweichenden Formen durch Analogie erklären. Gewisse Gegebenheiten scheinen jedoch gegen die Hypothese von Collinder und für die von Posti zu sprechen: für eine vom Schwund des $i$ oder $j$ der folgenden Silbe unabhängige Meta phonie also. Das gibt auch Wickman zu (Uppsala universitets årsskrift 1960 S. 41). Wie er feststellt, lässt sich keine Analogiequelle finden für die Metaphonie im Wort rig-

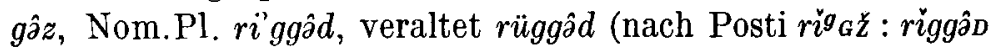
usw.), Sal. rügid ( $\sim$ fi. ruis, rukiit $<{ }^{*}$ ruyis, rukihit) (a.a.O. $39-40)$.

Im Entstehungsmechanismus der Metaphonie als solchem dürfte es nichts geben, was dazu zwänge, den Schwund des Verursachers der Metaphonie anzunehmen. Im Lappischen ist der Verursacher der Metaphonie allgemein nicht geschwunden, sowohl in der ersten Metaphonie des Urlappischen (z.B.

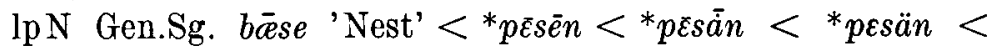
pesän, vgl. 2. Sg.Imp. bâsâ 'waschen' $<{ }^{*}$ pešesek $<{ }^{*}$ pè sę $k<$ 
*pesek) als auch in der im Süd- und Ostlappischen am deutlichsten erscheinenden zweiten Metaphonie. Eine Syn- oder Apokope eines zum Stamm gehörenden Vokals ist im Lappischen überhaupt eine relativ seltene Erscheinung; auch dort, wo es heute viele Formen mit Syn- und Apokope gibt, wie im Kolalappischen, scheint es sich um ein relativ junges Phänomen zu handeln. Auch die Oppositionen der Vokale der zweiten Silbe, die eine Metaphonie hervorriefen oder nicht, sind nicht immer neutralisiert worden. So weicht im Inari- und Skoltlappischen die Vertretung des ${ }^{*} e$ der zweiten Silbe stark $a b$ von der eines ${ }^{*} e$ der zweiten Silbe, und trotzdem sind die meisten Vokale der ersten Silbe vor einem $*_{\dot{e}}$ ganz anders vertreten als vor einem ${ }^{*} e$, z.B. lpI 1. Pl.Prs. topp'p̣p'

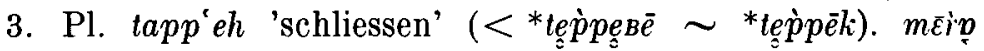

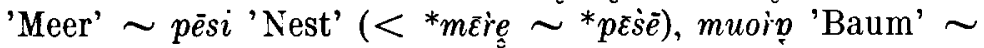

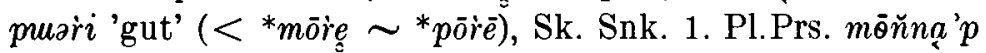

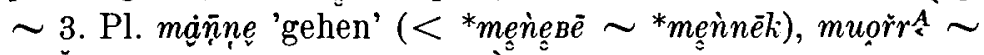

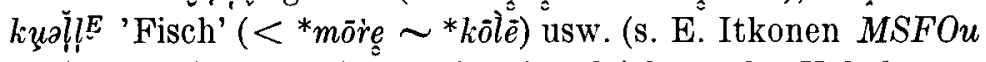
79 S. 8-43). Zwar gibt es eine Ausgleichung der Vokaloppositionen der zweiten Silbe im Süd- wie im Ostlappischen, doch zeigt bereits die geographische Verbreitung der Fälle, dass es sich um eine Entwicklung handelt, die jünger ist als die Metaphonie (s. S. 292, 295-298).

In diesem Zusammenhang muss auf den Unterschied hingewiesen werden, der zwischen einem allophonischen Metaphoniewechsel und einem durch Metaphonie bewirkten phonologischen Wechsel besteht. Der allophonische Metaphoniewechsel kann ganz unbemerkt bleiben. Bo Wickman hat angemerkt, dass die finnischen Phoneme $i$ und $e$ deutlich der Metaphonie unterliegen in Fällen wie silta 'Brücke' - Abl.Sg. siltä 'der' [siltta - silttä], kelta 'Gelb' - Abl.Sg. keltä 'wer' [kęlta keļtä] (a.a.O. 31). Der normale Benutzer des Finnischen kann den Unterschied zwischen hinterem und vorderem Allophon nur bemerken, wenn er besonders darauf achtet. Ein so geringer Metaphoniewechsel kann wohl kaum eine phonologische Funktion erhalten. Wenn aber der phonetische Unterschied zwischen den Allophonen so gross wird, dass er deutlich hörbar ist, können die durch Metaphonie erzeugten Allophone als 
Informanten hinsichtlich des Vokals (oder $j: s$ ) der folgenden Silbe aufgefasst werden: dann sind die Allophone als Quasiphoneme zu betrachten (vgl. S. 338). Auch in der Phonemisierung des Metaphoniewechsels im Livischen hat die quasiphonemische Stufe als wichtiges Zwischenstadium zu gelten. Es ist schwer vorstellbar, dass man von einem in einem bestimmten Stadium des Livischen herrschenden Verhältnis Nom.Sg. *tammi - Gen.Sg. *tamme durch Apokope direkt zu dem Verhältnis täm - tā̄ gekommen wäre. Wahrscheinlicher ist doch, dass sich der Unterschied zwischen dem vorderen und dem hinteren $a$ so stark entwickelt hatte, dass bereits eine Vorinformation über den Vokal der zweiten Silbe darin enthalten war, wodurch dieser als redundant schwinden konnte. Besonders deutlich wurde der quasiphonemische Charakter der metaphonischen Allophone in dem Fall, dass eins der Allophone mit einem anderen Phonem zusammenfiel. Wenn das vordere Allophon des livischen a mit dem in der Sprache ron jeher vorhandenen $\ddot{a}$ vor der Apokope zusammenfiel (*tämmi - $\left.{ }^{*} t a m m e\right)$, wurde die Differenz zwischen den einzelnen Allophonen des $a$ natürlich viel stärker empfunden, als wenn es in der Sprache kein $\ddot{a}$-Phonem gegeben hätte. Das chronologische Verhältnis des vor einem $i$ und $j$ der zweiten Silbe eingetretenen Wandels $\ddot{a}>e$ zu dem Wandel $a>\ddot{a}$ dürfte hier kaum von Bedeutung sein. Eine Überschneidung dürfte aber keine unbedingt notwendige Voraussetzung für die Phonemisierung der Metaphonie sein. Vor der Apokope konnte ebenso gut ein solches Verhältnis herrschen, dass das vordere Allophon irgendwo zwischen $a$ und $\ddot{a}$ lag (vielleicht $*_{t a m m i}$ - *tamme), doch so, dass es sich deutlich vom hinteren Allophon abhob; erst nach dem Vollzug der Apokope wäre dann das zu einem Phonem gewordene Produkt der Metaphonie zur Verdeutlichung der Opposition zusammengefallen mit $\ddot{a}$.

Obgleich in diesem Zusammenhang keine ausführlichen Messungen durchgeführt werden kömnen, scheint die livische Entwicklung grossenteils in gleicher Weise wie im Lappischen reguliert worden zu sein durch das Gleichgewicht von relativer Entropie und Redundanz. Die durch regressive Wirkungen verursachten qualitativen und quantitativen Wandel haben 
im Vokalismus der ersten Silbe und im darauffolgenden Konsonantismus eine beachtliche Zunahme der quasiphonemischen Oppositionen bewirkt, worauf automatisch ein Anwachsen der maximalen Entropie folgte. Die dadurch bewirkte übermässige Erhöhung der Redundanz förderte den Verfall der nichtersten Silben des Wortes, wodurch die Redundanz wieder ihren alten Wert zurückerhielt. Da die geschwundenen oder reduzierten nichtersten Silben suffixal waren, ging die morphologische Funktion der Suffixe in vielen Fällen auf Wechsel im Innern des Stammes über, wodurch auch die morphologische Methode des Livischen symbolische Züge erhielt.

Zur Stützung seiner Hypothese, wonach die Metaphonie nur vor einem geschwundenen Vokal der zweiten Silbe eingetreten sei, beruft sich Björn Collinder u.a. darauf, dass in einigen altnorwegischen Handschriften der Dat.Pl. vom Wort or 'Pfeil' (< späturn. *aru) arum lautet und nicht *orum und dass anstelle der Form *gofugr 'bedeutend' gafugr (Akk.Sg. aber gofgan) begegnet (Uppsala universitets arsskrift 1939: 1 S. 73). Der $a>q^{-U m l a u t}$ vollzog sich also nur vor einem geschwundenen $u$. Das auch aus anderen alten Denkmälern germanischer Sprachen bekannte Paradoxon, dass man die Metaphonie häufig erst dann zu bezeichnen begann, wenn der Laut, der sie hervorrief, geschwunden oder mit einem Laut zusammengefallen war, der keine Metaphonie verursachte, kann durchaus nicht ohne weiteres so ausgelegt werden, dass sich die Metaphonie erst dann richtig vollzogen hat. Es fragt sich, in welchem Mass die phonologische Stellung der Produkte der Metaphonie und die orthographischen Traditionen auf die Schreibweise der Wörter eingewirkt haben. Der Einfluss beider Gegebenheiten scheint z.B. in der Geschichte des Umlauts im Hochdeutschen sehr offenbar zu sein. Aus schriftlichen Quellen lässt sich der $a>e$-Umlaut im Althochdeutschen schon für das 8. Jahrhundert feststellen, doch wurde der Umlaut der anderen nichtpalatalen Vokale erst im Mittelhochdeutschen im 13. Jahrhundert bezeichnet. Dann war jedoch das $i$, das den Umlaut bewirkt hatte, entweder geschwunden oder mit $e$ zusammengefallen. Z.B. althochdeutsch gesti $\left(<{ }^{*}\right.$ gasti $)$ 'die Gäste', aber scôni $>$ mittelhochdeutsch 
schoene 'schön', wo ô erst dann zu $o e$ wurde, als $-i$ zu $-e$ geworden war. Dies bedeutet jedoch nicht, dass der Umlaut erst mit dem Schwinden des $i$ oder mit seiner Verwandlung in $e$ geschehen wäre. Schon die in althochdeutschen Sprachdenkmälern hin und wieder auftretenden Versuche, auch den Umlaut von anderen Vokalen ausser $a$ zu bezeichnen, zeigen, dass auch der Umlaut dieser Vokale alt ist. Der ganze Unterschied dürfte darauf beruhen, dass sich als Bezeichnung für das Resultat des Umlauts des $a$ der von jeher bekannte Buchstabe $e$ anbot, der als Zeichen des althochdeutschen Phonems $e$ verwendet wurde, während man für die Umlautvarianten der anderen Vokale kein bereits vorhandenes, geeignetes Graphem besass (W. Braune - W. Mitzka, Althochdeutsche Grammatik, 9. Aufl., Tübingen 1959, S. 59). Solange die Produkte des Umlauts Allophone des ursprünglichen Vokals waren, brauchte man für sie auch keine besondere Bezeichnung zu suchen. Dies wurde erst notwendig, als der Umlautwechsel aufgrund der Neutralisierung der Vokaloppositionen in der zweiten Silbe phonologisch wurde. Diese Ansicht dürfte zum ersten Mal von W. F. Twaddel in seinem Aufsatz »A Note on OHG Umlaut" (Monatshefte für den deutschen Unterricht 30 , 1938) geäussert worden sein; sie wurde dann später von zahlreichen Forschern gutgeheissen, die sich phonologisch mit der Metaphonie beschäftigt haben. Auch Herbert Penzl (Language 25 S. 224-225) hebt den Anteil der Phonemisierung des Umlautallophons an der Veränderung der Orthographie hervor. Er vertritt die Ansicht, Formen wie scôni 'schön', ubil 'übel, schlimm' seien schon im Ahdt. skö̀nI, $\ddot{u} b r l$ ausgesprochen worden. Der Wandel des $I, o, e$ und $a$ in der unbetonten Silbe zu a hätte verursacht, dass Oppositionen wie skōna - Adv. skōna (< scôni - scôno) nur aus der Vokalqualität der betonten Silbe hervorgingen. Möglicherweise wurden gemäss der schriftlichen Tradition die Vokale der betonten Silbe noch dann mit denselben Graphemen bezeichnet (scôni - scôno), als der Vokalunterschied der betonten Silbe in der gesprochenen Sprache schon distinktiv geworden war (sköna - skōna). Vgl. auch Moulton, Beiträge zur Geschichte der deutschen Sprache und Literatur 83, Tübin- 
gen 1961 S. 1-35; Kratz, Journal of English and Germanic Philology 59, Illinois 1960, S. 463-479).

Die Entwicklungsgeschichte der Metaphonie in den germanischen Sprachen kann hier nicht genau untersucht werden. Man ist sich jedoch heute weitgehend darin einig, dass der infolge regressiver Fernassimilation entstandene allophonische Metaphoniewechsel phonologisch geworden ist, wenn es in der folgenden Silbe gewisse Neutralisierungen von Vokaloppositionen oder einen Schwund von Vokalen gab. Eine Spezialuntersuchung wird zu klären haben, in welchem Masse der durch Metaphonie verursachte Allophonwechsel quasiphonemisch wurde, wieviel Information über die Vokalqualität der folgenden Silbe in den Quasiphonemen enthalten sein konnte und inwieweit der Übergang der Information auf die vorangehende Silbe Schwund oder Reduzierung der redundant gewordenen Vokale der folgenden Silbe verursacht hat. Der allgemeine Eindruck, den man von der Phonemisierung des Metaphoniewechsels in den verschiedenen germanischen Sprachen erhält, scheint darauf hinzuweisen, dass die quasiphonemische Entwicklungsphase und die damit verbundene Erhöhung der Redundanz ernstlich berücksichtigt werden muss.

Die Metaphonie wurde in keiner germanischen Sprache zum herrschenden morphologisch distinktiven Faktor; soweit aber die geschwundenen oder keine Distinktivität mehr besitzenden Elemente morphologisch gewesen sind, lässt sich ein Übergang der morphologischen Funktion auf den Vokal der vorangehenden Silbe und eine Zunahme der typisch symbolischen Flexion feststellen, z.B. dt. Vater - Pl. Väter, engl. foot - Pl. feet 'Fuss', isl. land - Pl. lond 'Land' (vgl. MSFOu 143 S. 21).

MikKo Korhonen 